\title{
Carolin Freier
}

\section{Soziale Aktivierung \\ von Arbeitslosen? \\ Praktiken und Deutungen eines neuen Arbeitsmarktinstruments}

[transcript] Gesellschaft der Unterschiede 
Carolin Freier

Soziale Aktivierung von Arbeitslosen?

Gesellschaft der Unterschiede | Band 38 
Carolin Freier (Dr. phil.) ist wissenschaftliche Mitarbeiterin am Institut für Arbeitsmarkt- und Berufsforschung. Die Soziologin promovierte an der FriedrichAlexander-Universität Erlangen-Nürnberg. 
Carolin Freier

\section{Soziale Aktivierung von Arbeitslosen?}

Praktiken und Deutungen eines neuen Arbeitsmarktinstruments

[transcript] 
Unter dem Titel: »Teilhabe in der Arbeitsgesellschaft? Praktiken und Deutungen eines sozial aktivierenden Arbeitsmarktinstrumentes « als Dissertation genehmigt von der Philosophischen Fakultät der Friedrich-Alexander-Universität Erlangen-Nürnberg.

Tag der mündlichen Prüfung: 29.06.2015

Vorsitzende des Promotionsorgans: Prof. Dr. Christine Lubkoll

Gutachter/in: Prof. Dr. Rainer Trinczek, Prof. Dr. Ingrid Artus

\section{(c) $\underset{\mathrm{BY}}{(\mathrm{NC}} \bigodot_{\mathrm{ND}}$}

Dieses Werk ist lizenziert unter der Creative Commons Attribution-NonCommercial-NoDerivs 4.0 Lizenz (BY-NC-ND). Diese Lizenz erlaubt die private Nutzung, gestattet aber keine Bearbeitung und keine kommerzielle Nutzung. Weitere Informationen finden Sie unter https://creativecommons.org/licenses/by-nc-nd/4.o/deed.de/.

Um Genehmigungen für Adaptionen, Übersetzungen, Derivate oder Wiederverwendung zu kommerziellen Zwecken einzuholen, wenden Sie sich bitte an rights@transcript-verlag.de

\section{(C) 2016 transcript Verlag, Bielefeld}

Die Verwertung der Texte und Bilder ist ohne Zustimmung des Verlages urheberrechtswidrig und strafbar. Das gilt auch für Vervielfältigungen, Übersetzungen, Mikroverfilmungen und für die Verarbeitung mit elektronischen Systemen.

\section{Bibliografische Information der Deutschen Nationalbibliothek}

Die Deutsche Nationalbibliothek verzeichnet diese Publikation in der Deutschen Nationalbibliografie; detaillierte bibliografische Daten sind im Internet über http://dnb.d-nb.de abrufbar.

Umschlagkonzept: Kordula Röckenhaus, Bielefeld

Druck: Majuskel Medienproduktion GmbH, Wetzlar

Print-ISBN 978-3-8376-3548-5

PDF-ISBN 978-3-8394-3548-9

Gedruckt auf alterungsbeständigem Papier mit chlorfrei gebleichtem Zellstoff. Besuchen Sie uns im Internet: http://www.transcript-verlag.de

Bitte fordern Sie unser Gesamtverzeichnis und andere Broschüren an unter: info@transcript-verlag.de 


\section{Inhalt}

$1 \quad$ Zusammenfassung |9

2 Maßnahmen Sozialer Aktivierung: Eine erweiterte Arbeitsmarktpolitik | 13

3 Theoretischer Zugang und Methode | 19

3.1 Forschungsfrage: Exploration und Analyse der Maßnahmen Sozialer Aktivierung | 19

3.2 Relevanz des Themas und Forschungsstand: Mitnichten eine quantité négligeable | 21

3.3 Forschungsleitende Annahmen | 25

3.3.1 Die Rolle der Arbeitslosigkeit in der Lohnarbeitsgesellschaft | 25

3.3.2 Die Arbeitsverwaltung als Schnittpunkt von Machtdiskurs und Subjektivität $\mid 29$

3.4 Studiendesign: Vorgehen zur Klärung der Forschungsfragen | 34

3.4.1 Wissenssoziologische Diskursanalyse als Analyseinstrument | 37

3.4.2 Grounded Theory als Orientierung für die Erhebung $\mid 42$

3.5 Geltungsbereich der Studie $\mid 45$

$4 \quad$ Aktivierungsdiskurs: Wandel der Arbeitsmarktpolitik |47

4.1 Der aktivierende Staat 49

4.2 »Menschen in Arbeit bringen«: Sicht auf die Leistungsbeziehenden $\mid 52$

4.2.1 Arbeitsmarktdienstleistungen für Kundinnen und Kunden |52

4.2.2 »Arbeitslosigkeit >bekommt ein Gesicht««: Aktivieren eines erweiterten Personenkreises | 57

4.2.3 Disziplinierungszuwachs in Folge der Klassifizierung in eine Erwerbsfähigen-Nichterwerbsfähigen-Dichotomie | 61

4.3 Unternehmerische Elemente der Arbeitsmarktpolitik | 64

4.3.1 Strategien zur Reduzierung der Erwerbslosigkeit $\mid 65$

4.3.2 Zumutbare Beschäftigungsvielfalt versus Bildung | 69

4.4 »Alles ist sozial, was Arbeit schaff $\ll$ : Soziale Teilhabe über Erwerb $\mid 72$

4.5 Hybride Momente des Aktivierungsdiskurses |74 
4.5.1 Bedarfsgemeinschaft und Familie:

Menschen sollen füreinander einstehen |75

4.5.2 Aktivierende Arbeitsmarktpolitik versus subventionierter Arbeitsmarkt | 77

4.6 Diskurstragende Institutionen in der Arbeitsverwaltung | 80

4.6.1 Dynamische Verwaltung in gelebten Organisationen 80

4.6.2 »Fördern und Fordern« als Motor der Aktivierung | 84

4.6.3 Die Akteurinnen und Akteure in der Beschäftigungsförderung |90

\section{Maßnahmen Sozialer Aktivierung:} Ziele, Genese und Funktionen | 97

5.1 Maßnahmen Sozialer Aktivierung: Eine definierende Darstellung | 97

5.1.1 Fallbeispiel einer sozialarbeiterischen U25-Maßnahme in sozialem Brennpunkt | 100

5.1.2 Fallbeispiel einer sozial aktivierenden Maßnahme mit Sportelementen für Jugendliche | 102

5.1.3 Fallbeispiel einer Maßnahme zum Heranführen an geordnete Familienstrukturen und -prozesse | 104

5.1.4 Fallbeispiel einer Maßnahme zur Bereitstellung psychisch-physischer Unterstützungsangebote sowie gesundheitsorientierter Beschäftigungsvermittlung | 105

5.1.5 Fallbeispiel einer Maßnahme für Wohnungslose | 107

5.2 Rechtlicher und finanzieller Rahmen für die Schaffung sozial aktivierender Maßnahmen | 110

5.2.1 Planung und finanzielle Mittel | 111

5.2.2 Entstehungswege der Maßnahmen Sozialer Aktivierung | 116

5.2.3 Rechtliche Realisierungsform und lokale Umsetzungen der Maßnahmen Sozialer Aktivierung | 131

5.3 Das Aktivcenter: Professionalisierung und Zeichen einer Bottom-up-Entwicklung | 136

5.4 Spannungsfelder in der Installierung von Maßnahmen Sozialer Aktivierung | 142

5.4.1 Abwägen zwischen Aktivierung und Wettbewerbsprinzip | 142

5.4.2 Garantie der Rechtskonformität versus Bürokratisierung | 146

5.4.3 Begrenzung durch die vermittlungsorientierte Budgetierung | 153

5.4.4 Die Eingliederungsquote begünstigt arbeitsmarktnahe Maßnahmen | 155

5.4.5 Verfestigte Arbeitslosigkeit: Eine arbeitsmarktferne Klientel | 158 
5.4.6 Bottom-up-Entwicklung Sozialer Aktivierung versus Top-down-Steuerung der Beschäftigungsförderung | 163

5.5 »Wir müssen an die Persönlichkeit heran«:

Maßnahmenfunktionen | 166

5.5.1 Institutionenspezifische Funktionen | 167

5.5.2 Typen der Aktivierung: Soziale Funktionen | 172

5.5.3 Sozialstaatliche Funktionen $\mid 207$

6 Resümee und Ausblick | 215

6.1 Rekapitulation der Forschungsfragen $\mid 215$

6.1.1 Typische Maßnahmenformen Sozialer Aktivierung | 215

6.1.2 Genese der Maßnahmen Sozialer Aktivierung | 216

6.1.3 Spannungsfelder im Maßnahmenkontext $\mid 218$

6.1.4 Funktionen der Maßnahmen Sozialer Aktivierung | 219

6.2 Diskussion $\mid 222$

7 Quellen- und Literaturverzeichnis $\mid 237$

$8 \quad$ Anhang $\mid 257$

8.1 Abkürzungsverzeichnis $\mid 257$

8.2 Übersicht über die geführten Interviews $\mid 260$ 



\section{Zusammenfassung}

Maßnahmen Sozialer Aktivierung werden in dieser Studie definiert als jene neuartigen arbeitsmarktpolitischen Instrumente, die in erster Linie nicht direkt in den Arbeitsmarkt integrieren, sondern vielmehr Alltagskompetenzen, soziale Fähigkeiten und soziale Teilhabe von sehr arbeitsmarktfernen Personen verbessern möchten, indem das (Wieder-)Erlernen basaler Fähigkeiten gefördert wird. Sie stellen damit eine, mit dieser Arbeit erstmals identifizierte und untersuchte, Form staatlichen Handelns in Deutschland dar, die auftritt, obwohl sie in ihrer Grundausrichtung der aktivierenden Arbeitsmarktpolitik (die direkt in den Arbeitsmarkt integrieren möchte) widerspricht. In solchen Maßnahmen - etwa einem Theaterworkshop - sollen beruflich unerfahrene Langzeitarbeitslose soziale Teilhabe und Tagesrhythmen erleben und erlernen. Ferner können Maßnahmen Sozialer Aktivierung beispielsweise auch Tanz- oder Sportelemente beinhalten sowie sozialarbeiterische Unterstützung in der Bearbeitung gesundheitlicher, finanzieller, sozialer oder familiärer Problemlagen bieten, um in langfristiger Perspektive Erwerbstätigkeit überhaupt erst zu ermöglichen.

Die Studie schließt eine Forschungslücke und trägt zum Verstehen des neuartigen arbeitsmarktpolitischen Instruments bei, indem sie das Phänomen definiert und untersucht. Die Gestaltung und Genese der Maßnahmen Sozialer Aktivierung werden exploriert und ihre Funktionen rekonstruiert. Zu diesem Zweck werden 1.) Texte aus dem politisch-rechtlichen sowie institutionellen Bereich der Arbeitsverwaltung und 2.) fünfundvierzig Experteninterviews mit Fachkräften von SGB-II ${ }^{1}$ - und Maßnahmenträgern in Deutschland ${ }^{2}$ analysiert. Ausgehend

1 Auch Grundsicherungsträger oder Jobcenter genannt.

2 Von der Autorin im Rahmen des Forschungsprojektes »Soziale Aktivierung - Social Activation« geleitet von PD Dr. Promberger am Institut für Arbeitsmarkt- und Berufsforschung (IAB) erhoben und ausgewertet. 
von der Annahme, dass die Untersuchung nur durch eine Rekonstruktion relevanter rahmender Diskurse möglich ist und unter Bezugnahme auf die bestehende wissenschaftliche Forschung zur Aktivierungspolitik, wird die Wirkmächtigkeit eines Teildiskurses des Aktivierungsdiskurses im Untersuchungsfeld angenommen. Innerhalb dieses Teildiskurses agiert die Arbeitsverwaltung und dessen vorherrschende Deutungen beeinflussen die Praktiken der AkteurInnen der Arbeitsverwaltung maßgeblich. Für die empirische Untersuchung dient demnach die Rekonstruktion des spezifischen politisch-rechtlichen Aktivierungsdiskurses mit den Instrumenten der Wissenssoziologischen Diskursanalyse als Referenzrahmen. Die Analyse dieses Teildiskurses ergibt, dass der Leistungsbezug von sogenannten erwerbsfähigen Hilfebedürftigen als Handlungsproblem definiert wird und die zeitnahe Integration in Erwerbstätigkeit als vornehmliches Ziel gilt. Die auf unmittelbare Erwerbsintegration ausgerichteten Instrumente der Beschäftigungsförderung werden jedoch einer langzeitarbeitslosen, sehr arbeitsmarktfernen Klientel kaum gerecht. Ebenso divergiert in der untersuchten Alltagspraxis der AkteurInnen der Arbeitsverwaltung das Subjektverständnis über die erwerbslosen Leistungsbeziehenden von deren Subjektposition als Kunden im Aktivierungsdiskurs. Die diskursiv-kommunikativen Praktiken der lokalen Arbeitsverwaltung reagieren mit einer Bottom-up-Implementierung von niederschwelligen, sozial-stabilisierenden Maßnahmen für Langzeitarbeitslose.

Die vorgelegte Arbeit setzt an diesem Befund an und untersucht, wie und warum Maßnahmen Sozialer Aktivierung entstehen, obwohl sie den Intentionen des Aktivierungsdiskurses widersprechen. Die vorliegende Analyse zeigt anhand von Fallbeispielen variierende Formen von Maßnahmen Sozialer Aktivierung. Typische Geneseformen geben Aufschluss darüber, welche AkteurInnen die Maßnahmen wie initiieren. Ein zentrales Ergebnis ist, dass nach einer Phase der Integration von sozial aktivierenden Elementen über offenere Maßnahmenformen aufgrund verstärkter Nachfrage von SGB-II-Trägern ein Standardinstrument zur sozialen Aktivierung eingeführt wurde. In diesem Kontext werden Spannungsfelder zwischen den im Aktivierungsdiskurs transportierten Zielen und den Anforderungen in der Praxis der Arbeitsverwaltung rekonstruiert.

Maßnahmen Sozialer Aktivierungen können meist keine zeitnahe Erwerbsintegration ermöglichen, vielmehr erfüllen sie institutionenspezifische, soziale und sozialstaatliche Funktionen. Sie gehen damit weit über die expressis verbis im SGB II und SGB III genannten Ziele der Arbeitsmarktintegration hinaus. Die vorliegende Studie identifiziert Maßnahmen Sozialer Aktivierung als ganzheitlich auf die Person gerichtete Förderung von Beschäftigungsfähigkeit. Auf Ebene der sozialen Funktionen werden fünf Integrationstypen rekonstruiert: Die Integration in die Ordnungen der Tätigkeit, einer erwerbstätigen Familie, der 
Arbeitsverwaltung, des gesellschaftlichen Miteinanders und des aktiven Subjekts. Mit dieser sozialen Integration in normative Strukturen finden zugleich eine Ausweitung des Aktivierungsdiskurses sowie eine Anpassung der Institutionen der Arbeitsverwaltung statt.

Insgesamt erweisen sich Maßnahmen Sozialer Aktivierung einerseits als auf die Person in ihrer Gesamtheit zielende Instrumente, die, gemäß den Individualisierungstendenzen der aktivierenden Arbeitsmarktpolitik, eine sehr arbeitsmarktferne Klientel zur Eigenaktivität und Selbstverantwortlichkeit befähigen sollen. Andererseits bedeutet die umfassendere Einsicht der arbeitsmarktpolitischen Institutionen in die Lebenswelt der Erwerbslosen jedoch auch geweitete Einflussmöglichkeiten des Staates. Obwohl soziale Aktivierung im Rahmen einer eher rekommodifizierenden Arbeitsmarktpolitik stattfindet, in deren Kontext Marktabhängigkeiten sich individualisieren und Individuen responsibilisiert werden, wirken diese Maßnahmen dekommodifizierend, indem den Teilnehmenden ein legitimer Status trotz Erwerbslosigkeit zuteilwird. 



\section{Maßnahmen Sozialer Aktivierung: Eine erweiterte Arbeitsmarktpolitik}

Ein Fallmanager des Jobcenters steht unter anderem vor der Aufgabe, auch solche langzeitarbeitslosen Erwachsenen zu betreuen, die als sehr weit vom ersten Arbeitsmarkt entfernt eingestuft werden und einen gewissen Fortschritt in der Eingliederung in den Arbeitsmarkt nachzuweisen. Doch dies erweist sich in solch >schwierigen Fällen` nicht immer als einfache Aufgabe. Fähigkeiten, Konstitution und Lebensweise dieser Klientel gelten als teilweise so unvereinbar mit den Voraussetzungen in einer Maßnahme zur zeitnahen Wiedereingliederung, dass etwa eine Zuweisung in eine Qualifizierungsmaßnahme von vornherein zum Scheitern verurteilt erscheint. Und so finden sich die angesprochenen Personen neben einem Bewerbungstraining auch in einem Theaterworkshop wieder. Was nun aber hat ein Theaterworkshop mit der effizienten Wiedereingliederung in den ersten Arbeitsmarkt zu tun?

Die Beschäftigung mit dieser Frage ist Kern der vorliegenden Arbeit. In einem solchen Workshop sollen nämlich die Teilnehmenden grundlegende Voraussetzungen für eine spätere Berufstätigkeit entwickeln. Dies soll zum Beispiel die Selbstwirksamkeit fördern, etwa über das Sprechen vor einer Gruppe oder die Schulung der Ausdrucksfähigkeit mittels Sprach- und Körpereinsatz. Auch ganz basale Aspekte finden sich in den Maßnahmenzielen: sich einen Tagesrhythmus aneignen, der die Maßnahmenteilnahme ermöglicht, sich vom Wohnzum Maßnahmenort bewegen, das äußere Erscheinungsbild sowie die innere Haltung (teilweise wieder) für die Maßnahme und so später den Arbeitsmarkt tauglich zu gestalten.

So richtet diese Studie ihren Blick auf ein neuartiges Instrument der Beschäftigungsförderung, das erwerbslose Personen im Leistungsbezug der Grundsicherung - meist sogenannte Langzeitarbeitslose - sozial aktiviert, indem die Maßnahme ihren Schwerpunkt auf persönlichkeitsstabilisierende und sozial integrierende Effekte legt. Diese Maßnahmenform wird von Fachkräften der SGB- 
II-Träger $^{1}$ wie auch von Maßnahmenträgern initiiert, eingekauft und mit Maßnahmenteilnehmenden ${ }^{2}$ beschickt. Dabei zeichnen Fallmanagerinnen und Fallmanager in Deutschland von einem Teil der Langzeitarbeitslosen ein Bild, in dem letztere ihre Hoffnung auf eine Erwerbsarbeit aufgegeben haben, häufig unter Suchterkrankungen leiden, verschuldet und stark vereinsamt sind. Die so beschriebenen Personen gelten als »stetig wachsender Sockel von Erwerbslosen, der auch in wirtschaftlichen Prosperitätsphasen zurück bleibe« (Promberger 2005). Plakativ titelte die SZ: »Langzeitarbeitslosigkeit - Einmal Hartz IV, immer Hartz IV « (Öchsner 2011). Die Aktivierungspolitik in Deutschland ist jedoch darauf ausgerichtet, alle erwerbsfähigen Leistungsbeziehenden der Grundsicherung zu aktivieren und eine Erwerbsaufnahme über Instrumente der Beschäftigungsförderung zu unterstützen. Orientiert an der britischen »Work first«policy setzt Deutschland in seiner aktivierenden Arbeitsmarktpolitik (Opielka 2004: 86) integrale Bestandteile europäischer Beschäftigungsstrategie um (BA 2006: 88).

Bei der oben beschriebenen Klientel setzt der Maßnahmentypus der Sozialen Aktivierung $^{3}$ an. Darunter fasst die vorliegende Untersuchung mittelbar arbeitsmarktbezogene Maßnahmen, denen persönlichkeitsstabilisierende und sozial integrierende Effekte zugeschrieben werden. Sie gehen weit über die expressis verbis im SGB II und III genannten Ziele der Arbeitsmarktintegration und Verbesserung der Beschäftigungsförderung hinaus. Da sie nicht vorrangig auf Erwerbsintegration fokussiert sind widersprechen sie den Grundzügen aktivierender Arbeitsmarktpolitik und werden daher formell stets als Vorstufe oder flankierendes Modul zur Arbeitsmarktintegration angesehen - nie als Selbstzweck. Es handelt sich hierbei also um ein neuartiges Instrument der Beschäftigungsförderung, das in dieser Studie hinsichtlich seiner Maßnahmeninhalte und Genese

1 Diese sind auch bekannt unter den Namen Grundsicherungsträger oder Jobcenter.

2 Zur sprachlichen Vereinfachung wird teilweise die männliche Form im Plural verwendet, um Personen beiderlei Geschlechts zu bezeichnen.

3 Der verwendete Begriff ist an einem 1996-2003 durchgeführten niederländischen Arbeitsmarktprogramm (»Social Activation«) orientiert, in dem Langzeitarbeitslosen mit wenig Aussicht auf eine Erwerbstätigkeit, alternative Wege gesellschaftlicher Partizipation ermöglicht werden sollten: soziale Kontakte, Tagesstruktur und Förderung des Selbstbewusstseins. Befristet von der Jobsuche freigestellt, erhielten die Teilnehmenden für ehrenamtliches Engagement ein Entgelt (Europäische Kommission 2007). 
exploriert sowie in seinen Funktionen ${ }^{4}$ systematisiert wird. Das Spektrum von Maßnahmeninhalten umfasst Kurse zur Rückengymnastik, Ernährung, Stressbewältigung oder Haushaltsführung, kulturelle Maßnahmen wie Tanzkurse und Theatergruppen, aber auch Schuldner- und Suchtberatung sowie sozialpädagogische Betreuung. Während soziale Aktivierung in den Niederlanden als Begriff und legitimes Instrument der Aktivierungspolitik eingeführt wurde, etablierte sich das neuartige Instrument in der bundesdeutschen Beschäftigungsförderung über eine arbeitsverwaltungsinterne Praxis. Diese Entwicklung, von der Verwaltungspraxis hin zum etablierten Instrument des Aktivcenters, wird in vorliegender Studie nachgezeichnet und in Bezug zum politisch-rechtlichen Aktivierungsdiskurs analysiert. Die auf dem Forschungsstand zur Aktivierungspolitik basierende Grundannahme ${ }^{5}$ ist, dass die Arbeitsverwaltung im Rahmen eines Aktivierungsdiskurses agiert und die Rekonstruktion des Teildiskurses für die Untersuchung notwendig ist. Betrachtet wird im Folgenden also nicht ein umfassendes diskursives Feld (Keller 2011), das aus mehreren und zum Teil konträren Diskursen zur Aktivierung besteht, sondern gezielt der hier so bezeichnete politischrechtliche Aktivierungsdiskurs, der durch seine Institutionalisierung in juristischen und innerorganisationalen Vorschriften direkt das Handeln der AkteurInnen der Arbeitsverwaltung bestimmt. Aufgrund seiner Bedeutung für die Handlungen der AkteurInnen wird die Formation des deutschen Aktivierungsdiskurses aus politischen, rechtlichen und internen Dokumenten der Arbeitsverwaltung rekonstruiert. Er beinhaltet Annahmen zum Staat, zur Erwerbstätigkeit, zu unternehmerischen Elementen der Arbeitsmarktpolitik und den Blick der Arbeitsverwaltung auf die Leistungsbeziehenden. Zur Diskursanalyse werden Interviews mit AkteurInnen der Arbeitsverwaltung in Beziehung gesetzt. Das empirische Material liefert Einblick in die Praxis- und Lebenswelt der Arbeitsverwaltung und ermöglicht die Deutungsmusteranalyse zu Aktivierung und Integration. Die hier gewählte Wissenssoziologische Diskursanalyse, die eine Diskursformation in Beziehung zu Deutungen und Praktiken von AkteurInnen setzt, geht über eine reine Dokumentenanalyse von Verwaltungsvorschriften hinaus. Das im Diskurs

4 Dabei steht der hier verwendete Funktionenbegriff nicht im Kontext der funktionalistischen Systemtheorie, sondern beschreibt in der vorliegenden Studie die Bedeutung der neuartigen Maßnahmenform auf institutionenspezifischer, sozialer und sozialstaatlicher Ebene (vergleiche Kapitel 3.4.1).

5 Vergleiche Kapitel 3.3.2 Die Arbeitsverwaltung als Schnittpunkt von Machtdiskurs und Subjektivität sowie Kapitel 3.4 Studiendesign: Vorgehen zur Klärung der Forschungsfragen. 
definierte Primärziel ist die Aufnahme einer Erwerbstätigkeit ( $\$ 1$ Abs. 2 SGB II, Deutscher Bundestag 2013b) bei einer gleichzeitigen Stärkung der Eigenverantwortung (»Hilfe zur Selbsthilfe« nach § 1 Abs. 1 SGB II), womit eine Individualisierung von Marktrisiken einhergeht. Dingeldey $(2007,2010)$ zeigt, dass in Deutschland, Dänemark und Großbritannien zwar unterschiedliche Aktivierungsvarianten existieren, doch bei allen Arbeitskraft rekommodifiziert wird, also sozialstaatliche Leistungen zurückgenommen werden und damit die individuellen Marktabhängigkeiten wachsen. Der Druck zur Erwerbsaufnahme ist demnach für Arbeitslose sowie für Arbeitende gestiegen.

Aktuell zeichnet der Arbeitsmarkt folgendes Bild: Die Zahl der Arbeitslosen im Bereich der Grundsicherung ist weiter zurückgegangen und lag seit der $\mathrm{Zu}$ sammenlegung von Arbeitslosenhilfe und Sozialhilfe 2005, im Jahr 2012 erstmals unter zwei Millionen (BA 2013e: 6). Gleichzeitig sind nur zwei von fünf Arbeitslosengeld II-Beziehenden arbeitslos (BA 2013e: 10) und Anstiege der Arbeitslosigkeit insgesamt alleine auf den Bereich der Arbeitslosenversicherung zurückzuführen (BA 2013e: 6). Diese Veränderungen können zum Teil als Folge der neuen Arbeitsmarktstrategie der Aktivierung verstanden werden, denn letzterer werden eine effizientere Arbeitsvermittlung, eine intensivere Stellensuche von Erwerbslosen sowie die Schaffung neuer Stellen durch die Arbeitsmarktflexibilisierung zugeschrieben (Klinger/Rothe/Weber 2013). Gleichzeitig nehmen jedoch Lohnungleichheit und atypische Beschäftigung zu (Klinger et al. 2013, Rhein 2013, Dörre et al. 2013). So zeigt sich, dass es einerseits Personen gibt, die selbst aktiv nach einem Lebensunterhalt suchen, jedoch dauerhaft oder in geringem Umfang prekär beschäftigt sind und damit existenzsichernder dauerhafter Beschäftigung bedürfen (Ludwig-Mayerhofer/Promberger 2008: 9). Obwohl sozialversicherungspflichtige Beschäftigungsverhältnisse quantitativ zugenommen haben und 2013 mit 41,84 Millionen den höchsten Stand seit 1990 aufweisen, ist die Arbeitslosigkeit leicht gestiegen, was die Bundesagentur für Arbeit mit geringeren Teilnahmezahlen an arbeitsmarktpolitischen Programmen begründet, denn damit wurden Personen von der sogenannten stillen Reserve in die offizielle Statistik zurückgeführt (BA 2014). Weiter abgenommen hat die sogenannte Unterbeschäftigung, die sich aus der Differenz des Erwerbspersonenpotenzials und der Zahl der Erwerbstätigen respektive der Summe aus Erwerbslosigkeit ${ }^{6}$

6 In der statistischen Berechnung werden Erwerbslose und Arbeitslose unterschiedlich erfasst, doch ihre Schnittmenge beträgt 60 Prozent. 
und Stiller Reserve ${ }^{7}$ ergibt (Fuchs et al. 2013: 8). Doch prognostizieren Fuchs und KollegInnen, dass die Arbeitslosigkeit trotz eines Beschäftigungsrekordes 2014 nur wenig sinken wird, da strukturelle Faktoren einen weiteren Abbau der Arbeitslosigkeit erschweren, so zum Beispiel Suchtfriktionen, aber auch eine ungenügende Passung der Qualifikationen von Erwerbslosen und vakanten Stellen (Mismatch) (Fuchs et al. 2013).

»Aus verschiedenen Gründen und für verschiedene Personengruppen besteht offensichtlich weiterhin ein gesellschaftlicher Bedarf nach solidarischer Absicherung, aber auch nach Arbeit unterhalb der regulären existenzsichernden Erwerbsarbeit sei es zur Resozialisation, Arbeitstherapie, Heranführung an den Arbeitsmarkt, Kontrolle oder einfach zur sozialen Einbindung von Menschen, die bei den gegenwärtigen Nachfragestrukturen und persönlichen Eigenschaften dauerhaft chancenlos auf reguläre Beschäftigung bleiben.« (Ludwig-Mayerhofer/Promberger 2008: 8)

Weiter steht die Erwerbslosigkeit in einer auf dem Arbeitsprinzip basierenden Gesellschaft in engem Zusammenhang mit der Frage nach sozialer Integration und Teilhabe, insbesondere jener Personen, die als arbeitsmarktfern klassifiziert werden. Wie diese Studie zeigt, fördern einerseits institutionelle Randbedingungen den Effekt, dass arbeitsmarktnahe Maßnahmen als Hauptstrategie angewandt werden und dadurch Personen, die eine niederschwellige und unter Umständen zeitintensivere Betreuung benötigen, eher vernachlässigt werden. Insofern deckt sich diese Studie mit dem Ergebnis, dass Schulden-, Sucht- und psychosoziale Beratung nicht in dem Umfang angeboten werden, wie es den Anforderungen der Klientel im SGB-II-Bezug entsprechen würde (Kaltenborn/Kaps 2013). Doch in der Implementierung von Maßnahmen Sozialer Aktivierung zeigt sich, dass andererseits die Notwendigkeit von niedrigschwelligen Angeboten für eine spezifische Klientel gesehen und entsprechende Instrumente auch installiert werden. Damit wird in einer langfristigen Perspektive die Erwerbsintegration fokussiert, der Aktivierungsbegriff jedoch um eine soziale Aktivierung erweitert, in der persönlichkeitsfördernde und alltagsstabilisierende Aspekte vordergründig bedeutsam sind. Damit wird auch der Diskussion um soziale Integration und Teilhabe von Erwerbslosen in der Arbeitsgesellschaft Rechnung getragen. Während dem Aktivierungsdiskurs eine dauerhafte öffentlich geförderte Beschäfti-

7 Mit dem Begriff der Stillen Reserve werden besonders entmutigte Menschen bezeichnet, die eine Arbeitssuche aufgegeben haben, jedoch bei guter Arbeitsmarktlage eine Beschäftigung aufnehmen. 
gung als unangemessen gilt, wird debattiert, ob ein dauerhafter öffentlich geförderter Beschäftigungsbereich individuelle Beschäftigungsfähigkeit fördern könnte und ein Mehr an Integrations- und Teilhabechancen ermöglicht.

Die Untersuchung der Maßnahmen Sozialer Aktivierung gliedert sich daher in folgende Teilbereiche:

In Kapitel 1 werden der theoretische Zugang der Arbeit und die verwendeten Methoden vorgestellt. In Kapitel 1 wird die Formation des Aktivierungsdiskurses sowie das heterogene Akteursfeld der Arbeitsverwaltung analysiert, über das Maßnahmen der Beschäftigungsförderung und Maßnahmen Sozialer Aktivierung installiert werden. Grundannahme ist, dass der Aktivierungsdiskurs, als politisch-rechtlicher Machtdiskurs, spezifische Deutungen und damit verbundene Implikationen für die Praxis der Arbeitsverwaltung mit sich führt. In Kapitel 1 werden Maßnahmen Sozialer Aktivierung als neuartige niederschwellige Maßnahmen für eine sehr weit vom Arbeitsmarkt entferne Klientel exploriert sowie deren Geneseformen dargestellt. Diese arbeitsmarktpolitischen Instrumente entstehen bottom-up durch eine verstärkte Nachfrage von Akteurinnen und Akteuren, die direkt mit SGB-II-Leistungsbeziehenden interagieren. Dabei werden Spannungsfelder bei der Installierung der Maßnahmen im Hinblick auf Interessen und Zielsetzungen sowie Handlungsbedingungen des Akteursfeldes gegenüber dem Aktivierungsdiskurs deutlich. Weiter werden über eine Analyse der Deutungsmuster von Aktivierung und Integrationsform institutionenspezifische, soziale und sozialstaatliche Funktionen in Maßnahmentypen Sozialer Aktivierung ersichtlich. In Kapitel 1 werden die Ergebnisse gesamthaft diskutiert. 


\section{Theoretischer Zugang und Methode}

Im Folgenden werden in Kapitel 3.1 die Forschungsfragen entwickelt und in Kapitel 3.2 die Bedeutung einer qualitativen Untersuchung von Maßnahmen Sozialer Aktivierung in den Kontext des Forschungsstandes gestellt. Die in Kapitel 3.3 dargestellten forschungsleitenden Annahmen zur Rolle der Arbeitslosigkeit in der Lohnarbeitsgesellschaft und der Arbeitsverwaltung als Form der Regierung bilden die Basis für diese Studie, deren Design in Kapitel 3.4 dargelegt wird. In Kapitel 3.5 wird der aus dem Forschungsdesign resultierende Geltungsbereich der hier formulierten Erkenntnisse eingegrenzt.

\subsection{ForsChUNGSFRAgE: EXPLORATION UND ANALYSE DER MASSNAHMEn SOZIALER AKTIVIERUNG}

Die Forschungsfragen dieser Studie sind auf die Exploration des Phänomens »Maßnahmen Sozialer Aktivierung« gerichtet, deren Genese sowie die Analyse der Funktionen, die sie für die Arbeitsverwaltung erfüllen. Letztere geschieht über Deutungsmusteranalyse zu Aktivierungs- und Integrationsformen, wie sie bei AkteurInnen der Beschäftigungsförderung zu finden sind. Die Entdeckung von sozial aktivierenden Maßnahmen (Kapitel 1), die in einem erweiterten Aktivierungsbegriff persönlichkeitsfördernde und identitätsstabilisierende Ziele verfolgen, um langfristig eine (Wieder-)Herstellung der Erwerbsfähigkeit zu bewirken, entsprechen nicht der Zielvorgabe einer kurzfristigen Arbeitsmarktintegration. Insofern führte die Entdeckung dieser Maßnahmen, die von der Autorin in der Kategorie der Maßnahmen Sozialer Aktivierung gefasst werden, zu folgenden zentralen Forschungsfragen: 
Im Bereich der Exploration der Maßnahmen:

1.) Wie schlägt sich die Anforderung einer niederschwelligen und ganzheitlichen, nicht primär auf Erwerbstätigkeit gerichteten Maßnahme Sozialer Aktivierung in ihrer inhaltlichen Ausgestaltung nieder?

2.) Wie entstehen die niederschwellig ausgerichteten Maßnahmen Sozialer Aktivierung im Umfeld eines auf kurzfristige Arbeitsmarktintegration ausgerichteten politisch-rechtlichen Diskurses?

3.) Welche Themen treten im zwangsläufig entstehenden Spannungsfeld zwischen dem Aktivierungsdiskurs und der Arbeitsverwaltungspraxis zu tage?

Im Bereich der Analyse der Funktionen:

4.) Welche Funktionen nehmen Maßnahmen Sozialer Aktivierung ein, wenn sie hinsichtlich der zugeschriebenen Zielsetzungen der Intervention, Subjektkonstitution, Tätigkeitsform und Aktivierungsform untersucht werden?

Um diese Fragen zu beantworten, gliedert sich der Aufbau der Studie entsprechend in eine Exploration und eine Analyse der Funktionen. ${ }^{1}$ Die Untersuchung der Maßnahmen wird dabei immer im Bezug zum Aktivierungsdiskurs vorgenommen.

1 In Kapitel 3.4 wird das methodische Vorgehen zur Bearbeitung der Forschungsfrage näher erläutert. 
Abbildung 1: Exploration und Analyse der Funktionen

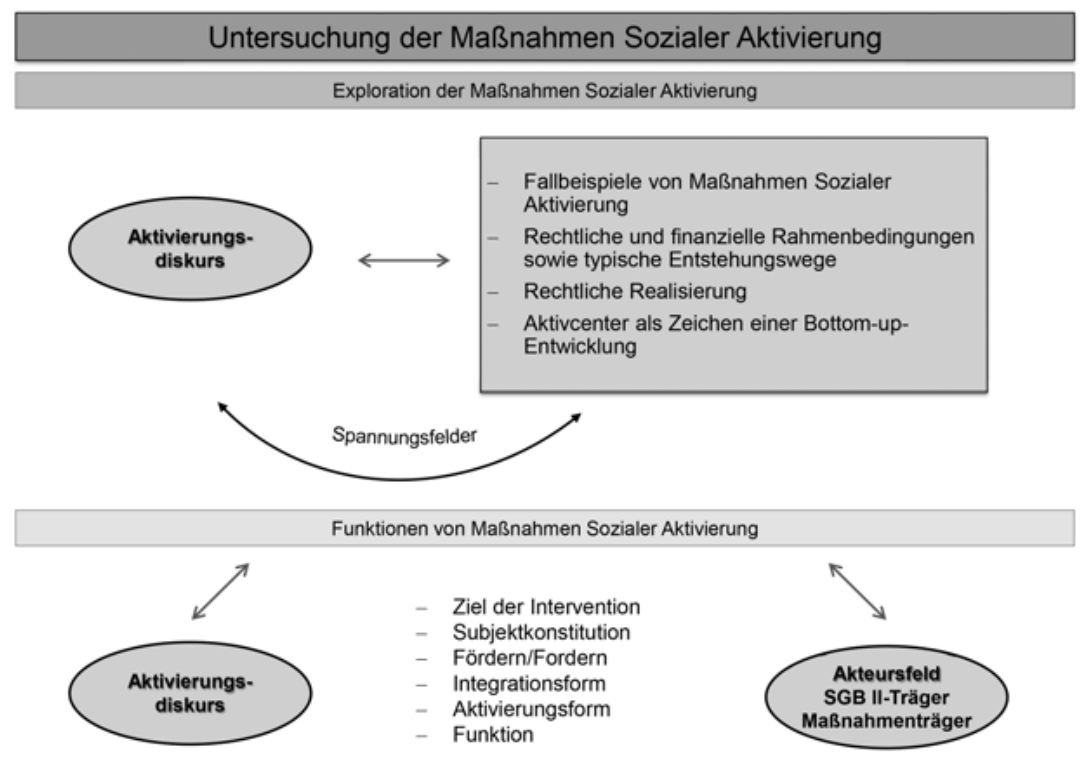

Quelle: Eigene Darstellung

Welche Bedeutung der Untersuchung von Maßnahmen Sozialer Aktivierung zukommt, wenngleich diese Maßnahmen quantitativ in dem Instrumentenpool der Arbeitsverwaltung keinen so bedeutenden Raum wie etwa die Arbeitsgelegenheiten nach $\S 16$ d SGB II einnehmen, wird nachfolgend ausgeführt.

\subsection{Relevanz des Themas und Forschungsstand: MITNICHTEN EINE QUANTITÉ NÉGLIGEABLE}

Die sozial aktivierenden Maßnahmen in den Niederlanden sind bereits formell von anderen Arbeitsmarktprogrammen unterschieden und so anhand spezifischer Beschäftigungsförderungsinstrumente leicht zu identifizieren. Will man sich hingegen in Deutschland einen Überblick über die Verbreitung dieser Maßnahmenform verschaffen, muss entlang möglicher Maßnahmeninhalte recherchiert werden. Die zeitweise ausschließlich latent praktizierten Maßnahmen können 
erst seit Einführung des Aktivcenters im September $2010^{2}$ direkt in den Controlling- und Verwaltungsprogrammen der Arbeitsverwaltung recherchiert werden. Mit Blick auf das Aktivcenter handelt es sich bei der sozialen Aktivierung in Deutschland rein quantitativ betrachtet um eine Randerscheinung: Während 2010 rund 26.000 und 2012 rund 17.000 Teilnehmende in Aktivcentern waren, befanden sich 2012 knapp ein Drittel der SGB-II-Leistungsbeziehenden (ohne Förderung der Berufsausbildung) in Arbeitsgelegenheiten (137.000) (BA 2013b: 17). Allerdings lässt sich die Anzahl der Maßnahmen Sozialer Aktivierung nicht eindeutig beziffern, da diese auch nach der Einführung des Aktivcenters teilweise in anderen Maßnahmenformen durchgeführt werden. Beispiele für andere $\mathrm{Maßnahmenformen} \mathrm{sind} \mathrm{die} \mathrm{oben} \mathrm{angeführten} \mathrm{Arbeitsgelegenheiten,} \mathrm{aber} \mathrm{auch}$ die Freie Förderung oder das Vermittlungsbudget. ${ }^{3}$

Doch der Stellenwert der Maßnahmen Sozialer Aktivierung wird insbesondere durch ihre Entstehung deutlich. In der bundesdeutschen Beschäftigungsförderung etablierte sich zuerst eine verwaltungsinterne Praxis der sozial aktivierenden Maßnahmennutzung auf der unteren Verwaltungsebene (bottom-up). Auch die Etablierung eines bundesweiten Standardmaßnahmenproduktes »Aktivcenter" nach § 46 I S.1 SGB III und ab dem 01.04.2013 im § 16 Abs. 1 SGB II i.V.m. §45 SGB II weist auf die Relevanz von Maßnahmen Sozialer Aktivierung hin. Das Aktivcenter entstand in Reaktion auf beständige Anfragen lokaler SGB-II-Träger 2010 - dieses Studienergebnis sei bereits vorweggenommen. Aktivcenter-Maßnahmen sind dezidiert niederschwellig angelegt und beinhalten zentrale Bausteine wie Stressbewältigung, Bewegung, gesunde Ernährung, Umgang mit Suchtproblematiken, die Stärkung des Selbstbewusstseins und zeichnen sich durch eine Teilzeitoption sowie die Möglichkeit unterweisungsfreier Zeiten aus. Die bloße Existenz der neuen Aktivierungsform in Deutschland ist bemerkenswert, da Integrationserfolge standardisiert an Vermittlungsquoten in Erwerbsarbeit gemessen werden und ein niederschwelliges Maßnahmenportfolio, das diesen Intentionen nicht entspricht, für Langzeitarbeitslose zuvor formal nicht existierte. Dabei wird die geänderte Rechtslage den Entstehungszusammenhängen der derzeit gültigen bundesdeutschen Sozialgesetzgebung durchaus gerecht, denn im SGB II finden sich sowohl arbeitsmarktpolitische als auch sozialintegrative Zielsetzungen - deshalb auch das strukturelle Dilemma von Sozi-

2 Zunächst verankert als Aktivcenter im § 16 I SGB II i.V.m. § 46 I S. 1 SGB III und ab dem 01.04.2013 im $\S 16$ Abs. 1 SGB II i.V.m. $§ 45$ SGB III.

3 Vergleiche Kapitel 5.2 Rechtlicher und finanzieller Rahmen für die Schaffung sozial aktivierender Maßnahmen. 
alverwaltung und Sozialarbeit (Harrach/Loer/Schmidtke 2000). Die hierdurch auftretenden Spannungen zwischen zentraler Sozialverwaltung und lokalen, therapeutischen Interventionspraktiken führen unter anderem zu stark variierenden Ausgestaltungen der Arbeitsmarktinstrumente. Maßnahmen Sozialer Aktivierung wurden im Rahmen der qualitativen Explorationsstudie zu »Sozialer Aktivierung - Social Activation» ${ }^{4}$ mit verschiedenen Rechts- und Finanzierungsgrundlagen von Arbeitsmarktinstrumenten gefunden. ${ }^{5}$ Parallel zum an Gesetzen und Anweisungen orientierten Wissen besteht eine von den AkteurInnen bestimmte Maßnahmennutzung, die nach Wahl der gesetzlichen Rahmung, Maßnahmenzielsetzung und -umsetzung variiert und mittels qualitativer Sozialforschung exploriert werden kann.

Die Bottom-up-Entstehung der Sozialen Aktivierung in Deutschland verweist auf die innerinstitutionelle Brisanz dieses Maßnahmentypus. Die bestehenden Vergleichsstudien zur aktivierenden Sozialpolitik (Eppel et al. 2012, KonleSeidl 2010; Fromm/Sproß 2008, Konle-Seidl/Eichhorst 2008a, Dingeldey 2007, 2010) treffen jedoch kaum Aussagen über die konkrete Ausgestaltung der Maßnahmen, die Deutungsmuster der AkteurInnen und den Themenkomplex der sozialen Teilhabe. Dies erklärt sich hauptsächlich dadurch, dass diese Studien primär als ökonometrische Evaluationen angelegt sind und die Wiedereingliederungsquoten in Erwerbsarbeit erforschen. Wie oben dargelegt, kann ein statistischer Zugriff die latente Praxis der Maßnahmen Sozialer Aktivierung vor Einführung des Aktivcenters 2010 ohnehin nicht erfassen. Für die Phase der offenen Nutzung in Deutschland gibt es bis dato noch keine analytischen Studien. Die vorliegende qualitative Studie identifiziert und analysiert nun die in Deutschland bislang unerforschte Maßnahmenpraxis in ihren unterschiedlichen gesetzlichen Realisierungsformen und legt damit eine neue Form der Aktivierung offen. Publizierte Studien über die Akteurslandschaft der Beschäftigungsförderung (Schulz 2010, Bode 2005, 2011, 2013) und die Rolle der beratenden Fachkräfte der Arbeitsverwaltung (Steinke et al. 2012, Schütz et al. 2011, Oschmiansky 2011, Schallberger/Wyer 2010, Ludwig-Mayerhofer/Behrend/Sondermann 2009, Hielscher/Ochs 2009, Ames 2008, Baethge-Kinsky et al. 2007, Raithel 2006) erleichtern die Einordnung des Untersuchungsgegenstandes, erklären jedoch weder den Entstehungsprozess, noch die Funktionen und Auswirkungen sozialer Akti-

4 Von der Autorin unter der Leitung von PD Dr. Promberger am IAB durchgeführt.

5 Vergleiche Kapitel 5.2 Rechtlicher und finanzieller Rahmen für die Schaffung sozial aktivierender Maßnahmen. 
vierung im Zusammenspiel der beschäftigungsfördernden Institutionen. Diese Forschungslücke vermag die vorliegende Studie zu schließen.

Die vorliegende Untersuchung zieht eine Linie zur Sozialstaatsforschung, indem sie an der Beschäftigungsförderung für arbeitsmarktferne Langzeitarbeitslose ansetzt, die sich im sozialen Handlungsraum der Sozialverwaltung bewegt. Die Frage nach sozialer Teilhabe von Langzeitarbeitslosen über den Weg der sozialen Aktivierung wird begleitet von der Diskussion um das Aktivierungsparadigma (Lessenich 2012a, 2009a, Koch/Kupka/Steinke 2009, Bude 2006, Wolf 2006) und behandelt gleichzeitig die Einflüsse des sozialpädagogisch orientierten Enabling-Ansatzes und die Debatte um Sozialarbeit im Kontext eines paternalistischen Sozialstaatskonzeptes (zum Beispiel Maier 2008, Lutz 2010, Kessl 2006). Auch wenn insbesondere die Sicht der Arbeitsverwaltung auf die Leistungsbeziehenden verfolgt wird, wird die Praxis der Arbeitsverwaltung von Prozessen der Subjektivierung und Subjektwerdung im Rahmen der institutionellen Behandlung von erwerbslosen Leistungsempfangenden begleitet. Erkenntnisse dieser Prozesse der Subjektivierung im Zusammenhang mit den Arbeitsmarktreformen werden in die Analyse einbezogen (Bröckling/Krasmann/Lemke 2000, Lessenich 2003, Maurer 2006). Ferner kann die Studie beantworten, ob beziehungsweise in welchem Ausmaß soziale Aktivierung als ein geeignetes Instrument von Expertinnen und Experten gesehen wird, um der Heterogenität der Klientel im SGB-II-Bezug gerecht werden zu können. Allerdings befindet sich die soziale Aktivierung in Deutschland erst im Entstehen und bedarf daher umso mehr einer intensiven sowie perspektivreichen wissenschaftlichen Auseinandersetzung.

Zusammengefasst zeigt sich in der Arbeitsverwaltung ein neuartiges Instrument der Beschäftigungsförderung, das persönlichkeitsstabilisierende und sozial integrierende Elemente beinhaltet, jedoch erstmals in dieser Studie wissenschaftlich untersucht wird. Durch die Anlage von sozial aktivierenden Maßnahmen in verschiedenartigen Maßnahmenformen sind eine aussagekräftige quantifizierende Darstellung und Analyse ausgeschlossen. Gerade die Bottom-up-Entstehung in der hierarchisch organisierten Arbeitsverwaltung, die vordringlich Erwerbsintegrationen intendiert, wirft Fragen zu Genese und Funktionen von Maßnahmen Sozialer Aktivierung auf, die hier analysiert werden. 


\subsection{Forschungsleitende AnNahmen}

Die Untersuchung der Maßnahmen Sozialer Aktivierung basiert einerseits auf den Annahmen zur Rolle der Arbeitslosigkeit in einer ökonomisch organisierten und auf dem Erwerbsprinzip basierenden Gesellschaft (Kapitel 3.3.1) sowie andererseits auf Annahmen zur Arbeitsverwaltung als Schnittpunkt von Machtdiskurs und Subjektivität (Kapitel 3.3.2), die in diesem Kapitel dargelegt werden.

\subsubsection{Die Rolle der Arbeitslosigkeit in der Lohnarbeitsgesellschaft}

Die Rolle der Arbeitslosigkeit wird im weiteren Verlauf über die Rekonstruktion der Randbedingungen sozialer Teilhabe der Bürgerschaft, die ökonomische Gestaltung von Gesellschaft und die Funktion des Sozialstaats als potentielles Instrument der Dekommodifizierung nachvollzogen.

Sozialstaaten ermöglichen ihrer Bürgerschaft über politische und soziale Rechte eine Integration in die Gesellschaft, indem Ressourcen der sozialen und beruflichen Teilhabe bereitgestellt werden. Die Programmatik des Wohlfahrtsstaates postuliert, dass soziale Inklusion nur auf politisch-staatlichem Wege zustande kommen kann, indem dieser seiner Bürgerschaft subjektive Rechte gewährleistet (Kaufmann 2003). Schon Thomas H. Marshall beschreibt systematisch die in den wohlfahrtsstaatlichen Einrichtungen verankerten sozialen Rechte, wie bürgerliche Freiheit, politische Mitbestimmung und soziale Teilhabe in seinem Konzept des social citizenship (Marshall 1992). Soziale Rechte gewährleisten demzufolge erst ein Existenzminimum respektive Mindeststandards sozialer und beruflicher Teilhabe, die über den staatsbürgerlichen Status erworben und garantiert werden und sonst weitgehend voraussetzungslos sind. Die Ausgestaltung der Rechte und Teilhabe differiert jedoch nach historischer und gesellschaftlicher Ausprägungsform.

In der »Arbeitsgesellschaft« (Opielka 2004: 86ff.) stellt das arbeitsmarktpolitische Hauptziel die Reproduktion einer Gesellschaft dar, deren Wohlstandssicherung und soziale Teilhabe auf dem Erwerbsprinzip basieren. Da die soziale Teilhabe in der Arbeitsgesellschaft insbesondere über das Erwerbsprinzip organisiert ist, bedingt Arbeitslosigkeit einen erschwerten Zugang zu den notwendigen Ressourcen für eine gelungene Chancengleichheit und sozial-gerechte Teilhabe. Robert Castel verortet in der um Arbeit gewobenen Regulierung ein Defizit, da diese in postindustriellen Gesellschaften ihr Integrationsvermögen verliert und eine neue Verwundbarkeit durch das "Ausfransen der Absicherungen« entsteht (2000: 29f.). Die Arbeitsverwaltung zielt auf eine Integration von erwerbslosen Sozialleistungsbeziehenden in den ersten Arbeitsmarkt und wirkt im Voll- 
zug von Beschäftigungsförderungsmaßnahmen insbesondere bei Langzeitarbeitslosen selbst auf eine soziale Teilhabe der Maßnahmenteilnehmenden hin. Diese Studie analysiert welche Facetten der sozialen Teilhabe in der Gesellschaft durch die Arbeitsverwaltung gefördert werden.

Die Arbeitsverwaltung agiert im Rahmen eines rechtlich-politischen Diskurses, der in seiner »innerweltlichen Objektivierung " (Keller 2008: 266) via Gesetzestexte und innerorganisationale Handlungsanweisungen das Akteurshandeln und die Strukturprinzipien, in denen sie sich befinden, mitbestimmt. Dieser Diskurs steht im Zeichen einer aktivierenden Arbeitsmarktpolitik. Er beinhaltet einerseits das der Arbeitsverwaltung inhärente Ziel der Aufnahme einer Erwerbstätigkeit der Leistungsbeziehenden und andererseits eine Stärkung der Eigenverantwortung, die charakteristisch für die aktivierende Arbeitsmarktpolitik ist. Dies bewirkt eine Individualisierung von Marktrisiken. Um diese Individualisierung von Marktrisiken zu beschreiben, ist der Blick auf die ökonomische Organisation der Gesellschaft von Nutzen. Die Herausbildung der Aktivierungspolitik ist an eine kapitalistische Gesellschaft geknüpft, die Arbeitskraft als marktförmige Ware handelt und sich als Lohnarbeitsgesellschaft begreift. Karl Marx arbeitet heraus, dass Arbeitskraft im kapitalistischen Wirtschaftssystem ebenso wie andere Waren als Gegenstand des Tausches gehandelt wird, in diesem Fall gegen Lohn auf dem Arbeitsmarkt (Marx 1968: 181ff.). Der doppelt freie Lohnarbeiter muss als freie Person über seine Arbeitskraft verfügen und frei von sonstigen Bedingungen seiner Reproduktion sein, die den Verkauf seiner Arbeitskraft erübrigen würden (Marx 1968: 183). Karl Polanyi, der in seiner Untersuchung The Great Transformation den Wandel von Gesellschaftsordnungen vom 18. bis Mitte des 20. Jahrhunderts untersucht, sieht in der Kapitalisierung von Arbeit und Boden (Kommodifizierung) eine große Gefahr: Menschliche Arbeitskraft und natürliche Ressourcen werden ihrer überwiegend gebrauchswertorientierten Bestimmung beraubt und dem selbstregulierenden Markt preisgegeben (Polanyi 1977). »Die angebliche Ware >Arbeitskraft< kann nicht herumgeschoben, unterschiedslos eingesetzt oder auch nur ungenutzt gelassen werden, ohne damit den einzelnen, den Träger dieser spezifischen Ware, zu beeinträchtigen.« (Polanyi 1977: 100) Dabei ist Arbeit seit der Moderne positiv konnotiert und nicht nur Mittel zur Sozialintegration, sondern auch identitätsbildend-subjektbestimmender Gegenstand (Freier 2013). Denn zu der Kommodifizierung der Arbeitskraft tritt die Bedingung hinzu, dass kollektive Sicherungssysteme wie die gesetzliche Arbeitslosenversicherung, Erwerbsausfälle verhindern, die die Selbsterhaltung gefährden (Polanyi 1977: 226). Der Sozialstaat schützt somit das Individuum mittels Arbeitsmarkt-externer Existenzsicherung (Dekommodifizierung nach Esping-Andersen 1990), indem er die Marktabhängigkeit von Arbeit vorüberge- 
hend (etwa bei Krankheit, Kindererziehung) oder dauerhaft (ab einem definierten Alter oder einer Erwerbsunfähigkeit) mindert. Gleichzeitig muss jedoch die Marktgängigkeit von Arbeitskraft fortwährend beibehalten beziehungsweise wiederhergestellt werden. Die aktivierende Arbeitsmarktpolitik in Deutschland rekommodifiziert Arbeitskraft, indem sozialstaatliche Leistungen zurückgenommen werden und individuelle Marktabhängigkeiten wachsen (Dingeldey 2007, 2010).

Doch der Arbeitsmarkt nimmt derzeit nicht alle Erwerbslosen auf und produziert soziale Spannungen: Die Erfolgsmeldung, dass die Zahl der Arbeitslosen im Bereich der Grundsicherung seit der Zusammenlegung von Arbeitslosenhilfe und Sozialhilfe 2005, im Jahr 2012 erstmals unter zwei Millionen (BA 2013e: 5f.) lag, kann auf einen gut funktionierenden Arbeitsmarkt schließen lassen. Doch fördern prekäre Beschäftigungsverhältnisse besonders in strukturschwachen Regionen Unsicherheiten und Spannungen im Zusammenhalt der Gesellschaft (Brinkmann/Dörre/Röbenack/Kraemer/Speidel 2006) sowie eine Individualisierung, die eine solidarische Gesellschaftseinstellung nicht befördern (Grimm/Hirseland/Vogel 2013). 2010 war einer von vier Beschäftigten atypisch ${ }^{6}$ beschäftigt (Egeler 2012). So deckt Arbeit im Niedriglohnbereich die Lebenshaltungskosten nicht ab und ein Aufstocken mit Sozialleistungen wird für einen größeren Anteil der deutschen Bevölkerung notwendig (Rhein 2013). Im Jahr 2012 sind nur zwei von fünf Arbeitslosengeld II-Beziehenden arbeitslos (BA 2013e: 10). Die erwerbstätigen SGB-II-Leistungsempfangenden gehen im Juli 2013 dabei größtenteils einer sozialversicherungspflichtigen Beschäftigung nach, sie sind geringfügig beschäftigt oder ein geringer Teil selbstständig:

67,8 Millionen von insgesamt 30,9 Millionen Beschäftigten gingen 2010 einer Teilzeittätigkeit mit bis zu 20 Wochenarbeitsstunden nach und 20,6 Prozent der Beschäftigten arbeiteten für einen Niedriglohn (Egeler 2012). 
Abbildung 2: Erwerbstätige Arbeitslosengeld II-Beziehende

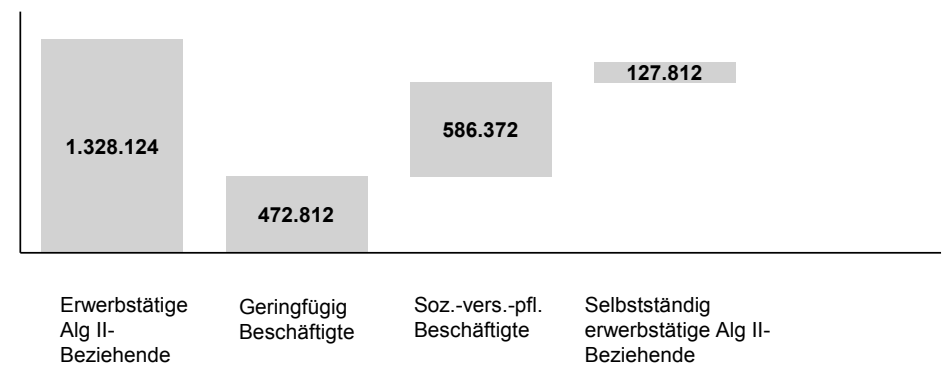

Quelle: Angelehnt an Statistik der Bundesagentur für Arbeit, Datenstand Juli 2013 (BA 2013d)

Brüchige Erwerbsbiografien in befristeten Arbeitsverhältnissen mit Phasen der Erwerbslosigkeit werden immer häufiger (Grimm et al. 2013). So heißt es im Jahresbericht zum SGB II der BA, dass Anstiege der Arbeitslosigkeit insgesamt alleine auf den Bereich der Arbeitslosenversicherung zurückzuführen sind (BA 2013e: 6). Weiter konstatiert Ingo Bode mit Bezug auf Martin Kronauer eine sozialstrukturell verfestigte Unterbeschäftigung, die durchschnittlich erwerbsfähige, wenn auch mitunter leistungsgeminderte, Personen an den Rand der Arbeitsgesellschaft drängt und eine hohe Dunkelziffer an Erwerbslosen, die aus der Zählweise der Erwerbslosigkeit resultiert (Bode 2011: 317). Die Bundesagentur betont »erhebliche Erfolge«, da in den vergangen fünf Jahren 700.000 Personen weniger arbeitslos und davon 400.000 Menschen in 2012 integriert wurden (BA 2013e: 27). Doch werden in einer öffentlichen Stellungnahme 2013 von DGB und paritätischem Wohlfahrtsverband mehr Mittel für Eingliederungsmaßnahmen nach den Kürzungen seit 2010 gefordert (Deutscher Bundestag 2013c). So stellte das BA-Vorstandsmitglied Heinrich Alt im Gespräch mit der Süddeutschen Zeitung den Mittelkürzungen bei Maßnahmen für insbesondere eine arbeitsmarktferne Klientel 2011 gegenüber »Je verfestigter die Arbeitslosigkeit ist, umso mehr müssen wir für Qualifizierung, Trainings, Schuldner- oder Suchtberatung ausgeben." (so Alt in Öchsner 2011) Denn: »Arbeitslosigkeit wird als Grundproblem der wirtschaftlichen und sozialen Integration demokratischer Gesellschaften angesehen « (Promberger 2005: 65) und ihr daher mit Arbeitsförderpolitik begegnet. Es entsteht ein Handlungsproblem, auf das die Regierung über die Arbeitsverwaltung reagiert. 
Zusammengefasst wird Arbeitslosigkeit unter anderem über die Arbeitsverwaltung mit dem Ziel deren Minderung und einer Stabilisierung des Arbeitsmarktes begegnet, da soziale Teilhabe der Bürgerschaft über das Erwerbsprinzip und den Sozialstaat in der Lohnarbeitsgesellschaft ermöglicht werden. Die aktuelle Arbeitsmarktpolitik setzt hierbei insbesondere auf eine Rekommodifizierung und Individualisierung von Marktrisiken.

\subsubsection{Die Arbeitsverwaltung als Schnittpunkt von Machtdiskurs und Subjektivität}

Die Arbeitsverwaltung wird in Anlehnung an Michel Foucault im Rahmen eines Verwaltungsstaates verortet. Hier wird die politische Ökonomie zur dominierenden Einflussgröße auf das Deutungs- und Handlungswissen. Legitime Deutungen, also objektiviertes Wissen vor allem im Hinblick auf ein diskursiv erzeugtes Handlungsproblem, werden in einem Machtdiskurs (re-)produziert, der sich in materialen Voraussetzungen und Folgen niederschlägt. Mit Bezug auf die diskurstheoretischen Überlegungen Reiner Kellers werden die in der Arbeitsverwaltung deutenden und interpretierenden AkteurInnen nicht allein als durch die soziale Konstruktion diskursiv-kommunikativer Praktiken determiniert gesehen. Vielmehr eröffnen sich Deutungs- und Handlungsspielräume, da AkteurInnen Wissensstrukturen nicht nur reproduzieren, sondern auch gestalten. Damit liegt die Arbeitsverwaltung im Schnittpunkt von Machtdiskurs und Subjektivität.

Foucault konstatiert ab dem Ende des 18. Jahrhunderts die Entwicklung eines Verwaltungsstaates, die er im Konzept der Gouvernementalität beschreibt. Gouvernementalität ist demnach unter anderem

»die Gesamtheit, gebildet aus den Institutionen, den Verfahren, Analysen und Reflexionen, den Berechnungen und den Taktiken, die es gestatten, diese recht spezifische und doch komplexe Form der Macht auszuüben, die als Hauptzielscheibe die Bevölkerung, als Hauptwissensform die politische Ökonomie und als wesentliches technisches Instrument die Sicherheitsdispositive hat.« (Foucault 2003: 820)

Diese Regierungsweise wird durch eine spezifische Wissensform der politischen Ökonomie ausgebaut, in der Analyseinstrumente wie die Statistik eine Steuerung der gesellschaftlichen Vorgänge erlauben. Die Regulierung und Konstitution der Bevölkerung ist nach Foucault insbesondere für die Bio-Macht zentral, die das 
Leben der Bevölkerung ${ }^{7}$ über Statistik analysiert und reguliert (Foucault 1976: 134ff.). »Die Abstimmung der Menschenakkumulation mit der Kapitalakkumulation, die Anpassung des Bevölkerungswachstums an die Expansion der Produktivkräfte und die Verteilung des Profits wurden auch durch die Ausübung der Bio-Macht in ihren vielfältigen Formen und Verfahren ermöglicht." (Foucault 1976: 136f.) Sozialtechnologische Instrumente, sogenannte Sicherheitsdispositive, analysieren die Bevölkerung in unterschiedlichen Facetten, ermöglichen etwa die wirtschaftliche Leistungsfähigkeit der Bevölkerung zu messen (Foucault 2003). Die sozialtechnologischen Instrumente werden durch den Einbezug der politischen Wissenschaft optimiert. So wirkt die Bio-Macht im Bereich des Arbeitsmarktes, indem Status und Form der Erwerbstätigkeit der Bevölkerung kontinuierlich statistisch untersucht und beobachtet sowie das institutionelle Vorgehen der Förderung von Erwerbstätigkeit in der Arbeitsverwaltung kontrolliert und reguliert werden.

Diese Regulation und Machtausübung können mit Foucault als Diskurs beschrieben werden: »Der Diskurs ist eine Reihe von Elementen, die innerhalb eines allgemeinen Machtmechanismus operieren. Darum muss man im Diskurs eine Folge von Ereignissen, zum Beispiel von politischen Ereignissen sehen, die der Macht als Vehikel dienen« (Foucault 2003: 595, zitiert nach Ruoff 2007: 99).

So wird in dieser Studie unter Bezug auf Foucault von einem Machtdiskurs in der Arbeitsverwaltung ausgegangen, der mit den Mitteln der Exklusion und Selektion operiert und parallel dazu legitimes Wissen organisiert (1991: 11ff.). Das bedeutet, dass der Diskurs Realität schafft, indem er historisch kontingente Sinnzusammenhänge sprachlich produziert. Analog zu diesen grundsätzlichen Ansätzen Foucaults wird in dieser Analyse nicht der Wahrheitsstatus von Wissen im erkenntnistheoretischen Sinne behandelt, sondern das diskursive Wissen als Effekt und Form von Macht (Keller 2006: 127). So wird das sozial konstruierte, typisierte und in unterschiedlichem Ausmaß legitime, objektivierte Wissen überwiegend in Diskursen gesellschaftlich produziert, legitimiert, kommuniziert und transformiert (Keller 2006: 115). Versteht man das im Diskurs erzeugte legitime Wissen als Effekt und Form von Macht (Keller 2006: 127) und nimmt somit die Perspektive einer Theorie der sozialen Konstruktion von Deutungs- und Handlungswissen ein, kann die Untersuchung der Wissensformation des Diskur-

7 Ferner beschreibt Foucault den modernen Staat als Regierungsstaat, der nicht mehr wesentlich durch seine Territorialität, sondern durch die Masse der Bevölkerung bestimmt wird (Foucault 2005a: 173). 
ses Aufschlüsse bieten über die Diskurseinflüsse auf die Beschäftigungsförderung. Die Verwaltungspraxis wird jedoch darüber hinaus durch die kommunizierenden und interpretierenden Fachkräfte gestaltet. Die Arbeitsverwaltung agiert über die Durchführung politisch-rechtlicher Vorgaben innerhalb des Aktivierungsdiskurses, produziert und reproduziert jedoch gleichzeitig auch das von diesem transportierte Wissen. Das diskursiv erzeugte Wissen und damit der Gebrauch von Typisierungen sind zwar sozial reguliert, doch nicht völlig determiniert, also immer auch durch Wahlmöglichkeiten einzelner Akteurinnen und Akteure gestaltbar (Keller 2013a: 89). Denn durch die gesellschaftliche Konstruktion werden diskursiv-kommunikative Praktiken im Handeln nicht nur reproduziert sondern auch verändert, da sie im Handeln ebenso durch die Interpretationsleistungen ihrer AkteurInnen bestimmt werden (Keller 2013a: 90).

Die Arbeitsverwaltung ist bürokratisch mit festgelegten und verbindlichen Strukturen organisiert. Schon durch Max Webers Bürokratiemodell, in dem Bürokratie als Instrument der rationalen und legalen Herrschaft fungiert (Weber 2005), wird deutlich, dass die Verwaltung insbesondere durch sozial geprägte Regeln gestaltet wird. Eine bürokratische Organisation bietet kraft berechenbarer Regeln Rechtssicherheit und Schutz vor Willkür. Die Bürokratie garantiert dabei die Einhaltung durch Paktierung oder Oktroyierung gesetzten Rechtes (Weber 2005: 160). Ferner stützt sich die Bürokratie auf formale Regeln, die durch Fachkräfte mit spezifischer Kompetenz (Weber 2005: 161) ausgeführt (und interpretiert) werden. So sind sowohl in der Setzung der Regeln als auch in der Ausübung der Regeldurchsetzung geteilte technische Regeln und sozial geprägte Normen von Bedeutung (Weber 2005: 161).

»Die bureaukratische Verwaltung bedeutet: Herrschaft kraft Wissen: dies ist ihr spezifisch rationaler Grundcharakter. Über die durch das Fachwissen bedingte gewaltige Machtstellung hinaus hat die Bureaukratie [...], die Tendenz, ihre Macht noch weiter zu steigern durch das Dienstwissen: die durch Dienstverkehr erworbenen oder >aktenkundigen< Tatsachenkenntnisse.« (Weber 2005: 165)

Nach Weber stellt die bürokratische Verwaltung durch Wissen somit in der legalen Herrschaft die Legitimität der Ordnung her. Für die vorliegende Untersuchung rückt das von Weber so bezeichnete Fach- und Dienstwissen der Arbeitsverwaltung in den Blick, anhand dessen sich die Strukturen der Praxis des Aktivierungsdiskurses nachzeichnen lassen.

Insgesamt sind Subjekte einerseits durch Semantiken und Praktiken von Diskursen beeinflusst, andererseits aber immer auch auslegende SprecherInnen des Diskurses. So sind die Fachkräfte der SGB-II- und Maßnahmenträger mit einem 
Interpretations- und Handlungsspielraum ausgestattet, der unterschiedliche Wege und Schwerpunktsetzungen in der Ausführung des politisch-rechtlichen Aktivierungsdiskurses zur Folge hat. Insofern sind gerade die Deutungsmuster der SGBII-Träger- und Maßnahmenträgerbeschäftigten für diese Studie von Interesse, denn erst vor dem Hintergrund dieser Deutungen lässt sich rekonstruieren, auf welche Annahmen und Funktionen letztlich die Etablierung von Maßnahmen Sozialer Aktivierung zurückzuführen ist.

Der politisch-rechtliche Aktivierungsdiskurs erzeugt als Machtdiskurs ein Wissen, das entlang des Kriteriums Erwerbsarbeit in Gesellschaft inkludiert (Selektion), während Arbeitslosigkeit zur Devianz generiert (Exklusion). ${ }^{8}$ Diskurse entwickeln parallel ein Ensemble an Mitteln, Mechanismen und Maßnahmen zur Bearbeitung von (selbst entworfenen) Handlungsproblemen und schaffen hierzu Infrastrukturen (Keller 2006: 136f.). ${ }^{9}$ Im Aktivierungsdiskurs ist das Beziehen sozialstaatlicher Leistungen bei Erwerbslosigkeit als Handlungsproblem konzipiert, dem mit institutioneller Disziplinierung und Förderung zur Erwerbsaufnahme begegnet wird. ${ }^{10}$ Ferner ist ein Diskurs als Praktik zu behandeln, welche mittels Sprache systematisch Gegenstände bildet (Foucault 1981: 74). Neben der Durchsetzung von Weltauslegungen haben Diskurse gesellschaftlich-materiale Voraussetzungen und Folgen (Keller 2006: 115). Beispielsweise etabliert der Aktivierungsdiskurs korrektive Disziplinierungspraktiken, wenn Erwerbslosigkeit eintritt. Die Menschen im Regelkreis des SGB II erfahren Begrenzungen und Möglichkeiten zur Teilhabe am sozialen, kulturellen und materiellen Leben, etwa über monetäre Mittel, die ihnen zur Verfügung gestellt werden.

Weiter legt die Arbeitsverwaltung den erwerbslosen Sozialleistungsempfangenden über die Interaktion und Bearbeitung des Handlungsproblems Erwerbslosigkeit bestimmte Subjektpositionen nahe, sie stellt sozusagen die Subjekte, auf die sie sich bezieht, selbst her. Denn Subjekte sind bei Michel Foucault keine ahistorische und vorsoziale Größe, sondern werden als Mitglieder einer Gesellschaft mit spezifischen Normen und Herrschaftsstrukturen über Sozialisationsprozesse geprägt und geformt:

8 Vergleiche Kapitel 4.4 »Alles ist sozial, was Arbeit schafft«: Soziale Teilhabe über Erwerb.

9 Wenn ein Diskurs agiert steht dies für den Zusammenhang von Struktur, AkteurInnen und Praxis, respektive die Wechselbeziehung zwischen objektivierter Wirklichkeit und den Artikulationspraktiken der AkteurInnen (Keller 2008: 264).

10 Vergleiche Kapitel 3.3.1 Die Rolle der Arbeitslosigkeit in der Lohnarbeitsgesellschaft. 
„Der zweite Aspekt [...] besteht darin, dass die Menschen im Laufe ihrer Geschichte niemals aufgehört haben, sich selbst zu konstruieren, das heißt ihre Subjektivität beständig zu verschieben, sich in einer unendlichen und vielfältigen Serie unterschiedlicher Subjektivitäten zu konstituieren. [...] Die Menschen treten ständig in einen Prozess ein, der sie als Objekte konstituiert und sie dabei gleichzeitig verschiebt, verformt, verwandelt - und der sie als Subjekte umgestaltet.« (Foucault 2005: 94)

Insbesondere in Überwachen und Strafen (Foucault 1994) und Der Wille zum Wissen (Foucault 1976) stellt Foucault einen Zusammenhang zwischen einer Disziplinarmacht und dem Individuum her. Er entwickelt in Überwachen und Strafen das Bild eines unterworfenen Subjekts, das erst durch Subjektivierungsund Objektivierungsprozesse über körperliche Disziplinierung, Normierung und Sanktionen zum Subjekt wird - im Rahmen von Macht/Wissen-Komplexen (Foucault 1994: 39). Für den Kontext der Arbeitsverwaltung kann analog angenommen werden, dass eine Norm definiert wird, zu der die Individuen in Beziehung gesetzt werden: »Hand in Hand mit dieser >wertenden` Messung geht der Zwang zur Einhaltung einer Konformität.« (Foucault 1994: 236) Butler macht hier auf die Ambivalenz des Begriffes Subjekt aufmerksam, das im eigentlichen Wortsinn immer auch »das Unterworfene« bedeutet: Nur durch die Unterwerfung unter ein allgemeines Gesetz und spezifische Normen, gewinnt das Individuum Autonomie und kann so zum Subjekt werden (Butler 2001). Denn durch Disziplinierung und Normierung wird dem Individuum in den durch die Machtwirkung determinierten Bereichen Handlungsmacht zugesprochen. Gleichzeitig beschreibt Foucault die Selbstherstellung des Subjektes über Technologien des Selbst. Diese Selbsttechnologien sind Strategien, die dem Subjekt dazu verhelfen sich in Denk- und Lebensweise so zu verändern, »dass er einen gewissen $\mathrm{Zu}$ stand des Glücks, der Reinheit, der Weisheit, der Vollkommenheit oder der Unsterblichkeit erlangt.« (Foucault 2005c: 968) Es besteht damit ein reziproker Prozess: »Die Bevölkerung tritt als Subjekt von Bedürfnissen und Bestrebungen, aber ebenso auch als Objekt in den Händen der Regierung hervor; der Regierung gegenüber weiß sie, was sie will, zugleich aber weiß sie nicht, was man sie machen lässt.« (Foucault 2000: 61) Das Subjekt ist demnach einerseits der Normierung und Herrschaft unterworfen, andererseits jedoch durch ein Bewusstsein und Selbsterkenntnis an seine Identität gebunden (Foucault 2005c: 275). Insofern ist 
die Gouvernementalität Modus der Machtausübung und der Subjektivierung. ${ }^{11}$ Auch im Aktivierungsdiskurs und in der Arbeitsverwaltung wird Macht ausgeübt und erwerbslose Leistungsbeziehende subjektiviert.

Zusammengefasst werden die Foucaultsche Theorie, die Theorie der sozialen Konstruktion sowie die methodologischen Annahmen von Keller genutzt, um die dem Aktivierungsdiskurs der Arbeitsverwaltung zugrunde liegenden Wissensstrukturen aufzudecken und die in ihm definierten Handlungsprobleme und auf diese reagierenden Infrastrukturen offenzulegen. Diese Wissensstrukturen bilden die Basis für die Analyse der Praxis sozial aktivierender Maßnahmen. Welche Subjektpositionen den erwerbslosen Leistungsbeziehenden zugewiesen werden, kann zudem über die Analyse des empirischen Materials rekonstruiert werden.

\subsection{STUdiendesign: VORGEHEN ZUR KLÄRUNG DER FORSCHUNGSFRAGEN}

Die Exploration des Phänomens soziale Aktivierung und die Analyse der Funktionen erfolgen anhand politischer, rechtlicher und interner Dokumente der Arbeitsverwaltung sowie leitfadengestützter Experteninterviews ${ }^{12}$ in SGB-II- und Maßnahmen-Trägern in neun kontrastierenden Regionen. Die empirische Untersuchung wurde von der Autorin am Institut für Arbeitsmarkt- und Berufsforschung (IAB) $)^{13}$ durchgeführt. In einer Wissenssoziologischen Diskursanalyse wurden Dokumente aus dem für die Arbeitsverwaltung handlungsleitenden rechtlich-politischen Aktivierungsdiskurs rekonstruiert. Die darin enthaltenen

11 Subjektivierung kann hier nicht auf der Handlungsebene betrachtet werden. Vielmehr vermag die Wissenssoziologische Diskursanalyse Subjektivierungen in historische Diskurse einzubetten und hineinwirkende Dispositive zu erfassen (Keller 2013b: 42).

12 Die Erhebung orientierte sich an der Grounded Theory (vergleiche Kapitel 3.4.2). Außerdem erstrecken sich die Experteninterviews auf verschiedene Entscheidungsebenen der Maßnahmengenese, um implizites Expertenwissen zu erfassen.

13 Das Forschungsprojekt wurde unter der Leitung von PD Dr. Promberger im Auftrag des Bundesministeriums für Arbeit und Soziales (BMAS) vom IAB konzipiert und durchgeführt. Die hier vorgelegten Interpretationen und Schlussfolgerungen liegen allein in der Verantwortung der Autorin, sie sind nicht Teil des Auftrags, der Projektergebnisse oder deren Veröffentlichungen und geben nicht die Meinung des IAB oder des BMAS wieder. 
Deutungsmuster zu Aktivierung und Integration bilden den Rahmen für die empirische Untersuchung. Aus der Empirie werden Maßnahmen-Fallbeispiele, eine Genesetypologie sowie stets im Kontext zum untersuchten Aktivierungsdiskurs die Deutungsmuster der AkteurInnen (in Arbeitsverwaltung und MaßnahmenAnbieter-Umfeld) von Aktivierung und Integrationsform herausgearbeitet und die Funktionen abgeleitet, welche die Maßnahmen in der Praxis der Arbeitsverwaltung einnehmen.

Diese explorative Forschungsfrage legt ein offenes und damit qualitatives Vorgehen nahe. Über die qualitative Studie sollen in einem wenig erforschten Feld Erkenntnisse erbracht werden. Ein quantitativ orientiertes Vorgehen würde dagegen ein klares Verständnis der Inhalte der Maßnahmen Sozialer Aktivierung, ihrer Genese und der Kenntnis der sprachlichen institutionellen Fassung dieser spezifischen Maßnahmenformen voraussetzen. Basierend auf den Interviewprotokollen wird in dieser deskriptiv analytischen Studie zunächst das Phänomen der Maßnahmen Sozialer Aktivierung exploriert, indem MaßnahmenFallbeispiele und typische Geneseformen im Kontext der institutionellen Strukturen sowie des politisch-rechtlichen Diskurses herausgearbeitet werden.

Um eigene Akzente der interviewten Akteurinnen und Akteure (aus der gesamten Institutionenkette) in der konkreten Maßnahmennutzung erfassen zu können, werden die Ebenen der Arbeitsverwaltung (Maßnahmenplanung, Arbeitsvermittlung und Fallmanagement) und der Maßnahmenträger ${ }^{14}$ betrachtet. Dabei werden die Verwaltungspraktiken auf Ebene der SGB-II- und Maßnahmenträger untersucht im Hinblick auf: Vorkommen, Entstehungswege, mit den Maßnahmen verbundene Intentionen und Problemfelder sowie Entwicklungen im Maßnahmen-Anbieterfeld. ${ }^{15}$ Die Genese und Gestalt sozialer Aktivierung bieten Aufschluss über den lokalen Umgang mit den Anforderungen des politischrechtlichen Diskurses mit der zu aktivierenden Klientel sowie die Entwicklungen des Anbieterumfeldes von Aktivierungsmaßnahmen. Es zeichnet sich ein Spannungsfeld zwischen dem Aktivierungsdiskurs und den Maßnahmen Sozialer Aktivierung ab.

Die Existenz der seit einigen Jahren praktizierten Aktivierungspolitik ist bereits wissenschaftlich anerkannt und zahlreiche Arbeiten setzen sich mit deren

14 Maßnahmenträger werden von SGB II-Trägern mit der Maßnahmendurchführung betraut.

15 Siehe Kapitel 5 Maßnahmen Sozialer Aktivierung: Ziele, Genese und Funktionen. 
Wirkung für den Sozialstaat und den Arbeitsmarkt auseinander. ${ }^{16}$ Prämisse der vorliegenden Studie ist, dass die Aktivierungspolitik und das verbundene diskursive Feld um Aktivierung das Handlungsfeld der Arbeitsverwaltung rahmen. Existenz und Wirkmächtigkeit des Aktivierungsdiskurses werden demnach, basierend auf dem wissenschaftlichen Kenntnisstand, angenommen. Um das Phänomen der sozialen Aktivierung untersuchen zu können, wird infolgedessen der politisch-rechtliche Aktivierungsdiskurs beschrieben. Die Wissenssoziologische Diskursanalyse ermöglicht Verstehen sowie Codierung und damit eine detailliertere Beschreibung des für die Arbeitsverwaltung bedeutsamen Aktivierungsdiskurses. Diese Rekonstruktion basiert auf relevanten Dokumenten für die Arbeitsverwaltung, die in Kapitel 1 skizziert werden. Daneben berücksichtigt die Rekonstruktion wissenschaftliche Erkenntnisse, um den Forschungsstand in die Analyse miteinzubeziehen. Die qualitativen Experteninterviews liefern Einblick in die Lebenswelt der Arbeitsverwaltung und ermöglichen eine Deutungsmusteranalyse zu Aktivierung und Integration.

Um die Funktionen zu analysieren werden verschiedene Typen von Maßnahmen Sozialer Aktivierung abgeleitet und innerhalb des zuvor analysierten Aktivierungsdiskurses sowie des Akteursfeldes kontextualisiert. Die Rekonstruktion von Deutungsmustern von Aktivierung sowie Integration gibt darüber hinaus Aufschluss über den Umgang mit dem Problem der sozialen Teilhabe von arbeitsmarktfernen Langzeitarbeitslosen in der Arbeitsverwaltung und im Anbieter-Umfeld. Es gilt die Entscheidungsmaximen und Deutungsmuster in den spezifischen Funktionsbereichen der ExpertInnen zu rekonstruieren (Bogner/Menz 2009: 70). Eine wissenssoziologisch-orientierte Deutungsmusteranalyse bietet sich an: Mittels Deutungsmustern kann sowohl die subjektive Ebene, im Rahmen von Vorstellungen der ExpertInnen, untersucht werden, wie auch die Verwaltungspraxis von Langzeitarbeitslosen auf der intersubjektiven Ebene. Denn die individuelle Handlungsorientierung wird als abhängig von den sozial geteilten Sinnzuschreibungen verstanden und ermöglicht den ExpertInnen Komplexitätsreduktionen: »Deutungsmuster stellen eine kulturelle, kollektiv bzw. überindividuell (re-)produzierte Antwort auf objektive, Handlungsprobleme aufgebende gesellschaftliche Bedingungen dar« (Meuser/Sackmann 1992: 15). Die Analyse

16 Z.B. für die Bedeutung der Sozialstaatlichkeit (Bode 2004), (Bode 2013), (Lessenich 2009b), (Lessenich 2012b), (Bieback 2009), (Dingeldey 2007), (Dingeldey 2010), für die Wirkungsanalysen (Bernhard/Wolff 2008), (Konle-Seidl/Eichhorst 2008), (KonleSeidl 2008). Eher auf die Funktionsweise der Arbeitsverwaltung im Rahmen der Aktivierungspolitik zielen (Koch et al. 2009), (Schütz 2012) und (Schönig 2006). 
der Deutungsmuster liefert Befunde über Aktivierungsformen, Integrationsförderungen der Arbeitsverwaltung und sozialstaatliche Funktionen der Maßnahmen Sozialer Aktivierung. Das Forschungsunterfangen präzisiert damit die Nahtstelle sozialer Teilhabe, insbesondere im Bereich der Langzeitarbeitslosen.

Zusammengefasst gewährt die Untersuchung der lokalen Ausgestaltungen der Beschäftigungsförderung, in Reaktion auf unterschiedliche Problemlagen der Arbeitslosen und Arbeitsmarktlagen, Einblick in kaum beachtete und doch unmittelbar wirksame Verwaltungspraktiken der sozialen Aktivierung. Die Interpretation der Deutungsmuster zur Aktivierung und zu Integrationsformen in der Arbeitsverwaltung und im Anbieterumfeld wird darüber hinaus Befunde zur Förderung sozialer Teilhabe von Langzeitarbeitslosen und zum institutionellen Umgang der Arbeitsverwaltung mit Sockelarbeitslosigkeit in einer Lohnarbeitsgesellschaft herausarbeiten.

\subsubsection{Wissenssoziologische Diskursanalyse als Analyseinstrument}

Die Exploration und Analyse der Maßnahmen Sozialer Aktivierung wird über eine Interviewauswertung mit AkteurInnen der Arbeitsverwaltung vorgenommen. Denn das neuartige beschäftigungspolitische Instrument entwickelte sich zunächst latent durch das Wissen und die Praktiken der AkteurInnen und wurde später zu einem Standardinstrument. Dabei vollziehen sich Wissenskonstruktion und Handeln der AkteurInnen immer im Rahmen des politisch-rechtlichen Aktivierungsdiskurses, so die Grundannahme der vorliegenden Studie. Der Diskurs beeinflusst über gesetzliche Vorgaben und innerorganisationale Handlungsanweisungen den Handlungsspielraum. Gleichzeitig sind die AkteurInnen der Arbeitsverwaltung durch ihr Fachwissen, Interpretationen und Interaktionen dazu befähigt, ihren Handlungskorridor auszuloten und auf den Aktivierungsdiskurs rück zu wirken. Hier dokumentieren sich auch divergierende Deutungsmuster von Aktivierung und Integrationsformen. Daher werden die Deutungsmuster aus der Praxis der Arbeitsverwaltung in Bezug zum Aktivierungsdiskurs diskursanalytisch herausgearbeitet.

Genese, Gestalt und Funktionen von Maßnahmen Sozialer Aktivierung werden daher mit dem Fokus auf die Deutungen und Praktiken der AkteurInnen analysiert (Kapitel 1) und durch eine Analyse der rahmenden Diskursformation kontextualisiert (Kapitel 1). Diese beiden Perspektiven miteinander zu vereinbaren, vermag die Wissenssoziologische Diskursanalyse nach Keller (WDA). Denn die WDA kann nicht nur umfassende historische Diskursfelder analysieren, wie dies Michel Foucault vornehmlich praktizierte, sondern bietet eine fundierte Metho- 
de, um spezifischere Teildiskurse zu verstehen und codieren. Durch die Wissenssoziologische Basis ist neben dem Verstehen des Diskurses ebenfalls das Erfassen von Deutungen und Praktiken der AkteurInnen mit dieser Methode möglich. Zweifellos operiert die WDA hier mit unterschiedlichen Wissensbegriffen, die sich zwischen Foucault und Berger/Luckmann bewegen: intersubjektiv geteiltes Wissen in diskursiven Formationen, drücke sich nach Keller in einzelnen Dokumenten aus, könne jedoch auch für ein Ensemble einzelner Aussagen stehen (Angermüller 2005:30). Daher wird in der vorliegenden Studie nicht der Anspruch erhoben, den Aktivierungsdiskurs in seiner Gesamtheit zu rekonstruieren. Wie zu Beginn des Kapitels 3.4 ausgeführt, wird auf Basis wissenschaftlicher Kenntnisse ein Aktivierungsdiskurs vorausgesetzt. Ein für die Arbeitsverwaltung relevanter Teildiskurs wird hier rekonstruiert, um die Deutungsmusteranalyse zu Aktivierung und Integration zu kontextualisieren.

In einem ersten Schritt wird die Formation des Aktivierungsdiskurses, als politisch-rechtlicher Machtdiskurs der aktivierenden Arbeitsmarktpolitik mittels Wissenssoziologischer Diskursanalyse untersucht. Als Diskurs wird ein »Komplex von Aussageereignissen und darin eingelassene Praktiken, die über einen rekonstruierbaren Strukturzusammenhang miteinander verbunden sind und spezifische Wissensordnungen der Realität prozessieren« (Keller 2008: 235) verstanden. Damit wird die Foucaultsche Diskursanalyse in der WDA ${ }^{17}$ um die Analyse gesellschaftlicher Wissensverhältnisse und Wissenspolitiken erweitert (Keller 2013a: 90). Der Diskurs wird auf seine Erscheinungsweise vornehmlich über Inhalte, also die Wissenskonstruktion analysiert.

In interpretativer Erschließung werden die Aussageereignisse zerlegt und auf, dem Material entnommene, Kategorien bezogen. Untersucht wird 1.) die Diskursstruktur (Kapitel 1), also die Formation des Aktivierungsdiskurses bezogen auf seine Deutungsmuster zur Staatlichkeit (Kapitel 4.1), zu der Staatsicht auf die Leistungsbeziehenden (Kapitel 4.2), zu unternehmerischen Elementen der Arbeitsmarktpolitik (Kapitel 4.3) und zur Arbeit respektive (Wieder-)Herstellung von Arbeit über Aktivierung sowie die damit verbundene soziale Integration

17 Die Wissenssoziologische Diskursanalyse nach Keller versteht sich als sozialwissenschaftliches Forschungsprogramm und möchte die eher strukturtheoretisch angelegten Annahmen der Wissenssoziologie beziehungsweise Diskurstheorie von Foucault in die Tradition der handlungstheoretischen Wissenssoziologie stellen (Letztere basiert wiederum auf Berger/Luckmann und der interpretativen Soziologie) (Keller 2008: 187). 
(Kapitel 4.4). Exemplarische Widersprüche im Aktivierungsdiskurs weisen auf eine Vereinnahmung widerstreitender Positionen durch den Diskurs hin (Kapitel 4.5). Ferner wird der Aktivierungsdiskurs über Institutionen der Arbeitsverwaltung und beauftragter Dritter im Bereich der Maßnahmen Sozialer Aktivierung ausgeführt. Daher wird 2.) das diesbezügliche Praxisfeld des Aktivierungsdiskurses (SGB-II- und Maßnahmenträger von Maßnahmen Sozialer Aktivierung) untersucht (Kapitel 4.6).

Die Analyse des Aktivierungsdiskurses beschränkt sich zeitlich auf die Jahre 2001 bis 2013 und inhaltlich auf ausgewählte Texte aus dem politisch-rechtlichen sowie institutionellen Bereich der Arbeitsverwaltung ${ }^{18}$, die in Kapitel 1 analysiert werden. Herangezogen werden dafür Textsorten, die die materielle und ideelle Infrastruktur des Diskurses (Maßnahmenbündel, Regelwerke etc.) abbilden, das sogenannte Dispositiv, durch das ein Diskurs (re-)produziert wird und Effekte erzeugt werden (zum Beispiel Gesetze, Verhaltensanweisungen) (Keller 2008: 235). Denn diese geben auch Auskunft über den nutzbaren Handlungsspielraum der AkteurInnen der Beschäftigungsförderung. Der hier analysierte politisch-rechtliche Aktivierungsdiskurs vollzieht sich in einem breiten Diskursfeld zum Thema Aktivierung. So wird der analysierte Diskurs von Diskursen mit widerstreitenden Annahmen zur Aktivierung im Diskursfeld flankiert. Der Schwerpunkt wurde in der vorliegenden Studie jedoch auf diejenigen Diskurspositionen gelegt, die die Handlungskorridore des Akteursfeldes deutlicher prägen. Denn obschon der politisch-rechtliche Aktivierungsdiskurs in der Gesellschaft neben anderen Diskursen existiert, kann er für sich objektive Gültigkeit beanspruchen, da er in der Gerinnung zur Rechtsform verbindlich ist. Das Recht fungiert als Instrument der Sozialkontrolle und Quelle normativer Steuerung - es ist am stärksten formalisiert, verfügt über Exekutiv-Institutionen und besitzt damit einflussreiche Mittel sozialer Sanktionierung (Peuckert 2002: 106).

Bereits während der Darstellung der Maßnahmen Sozialer Aktivierung (Kapitel 5.1 bis 5.5) wird in einem zweiten Schritt das Praxisfeld der Arbeitsverwaltung zur herausgearbeiteten Aktivierungsdiskursformation in Bezug gesetzt. Eine besonders verdichtete Bezugnahme erfolgt in der Analyse der Funktionen (Kapitel 5.5). Maßnahmen Sozialer Aktivierung hinsichtlich ihrer Funktionen zu analysieren, meint hier, den Maßnahmen inhärente Bedeutungen auf institutionenspezifischer, sozialer und sozialstaatlicher Ebene. Diese Bedeutungen sind nicht allein durch intentionale Zieldefinitionen innerhalb des Aktivierungsdiskurses oder der AkteurInnen der Arbeitsverwaltung zu erklären. Vielmehr han-

18 Eine detaillierte Beschreibung des Textkorpus findet sich in Kapitel 1, S.41f. 
delt es sich häufig um nicht-intendierte Effekte, die in der Analyse deutlich werden. Äquivalent zur Organisationsforschung, in der Organisationsziele ihren Funktionen zugeordnet werden (Preisendörfer 2011: 62f.), werden die Deutungen des Aktivierungsdiskurses den Deutungen und Praktiken des Akteursfeldes der Arbeitsverwaltung gegenübergestellt. Inhaltlich grenzt sich die Studie vom Funktionsbegriff der funktionalistischen Systemtheorie ab, wie sie klassisch bei Talcott Parsons, Robert K. Merton oder Emile Durkheim verwandt wird.

Zunächst werden institutionenspezifische Funktionen basierend auf ihren im Aktivierungsdiskurs vorgesehenen Funktionen und den zugeschriebenen Maßnahmeneffekten der AkteurInnen rekonstruiert. Die sozialen Funktionen werden über einen systematischen fallübergreifenden Vergleich der Deutungsmuster als Typen der Aktivierung abgeleitet, die den Aktivierungsdiskurs in je spezifischer Weise ausführen. Im Rahmen des typenbildenden Verfahrens wurden die Deutungsmuster der Integrationsform, der Aktivierung, das Maßnahmenziel, die Sicht der Akteurinnen und Akteure, die Elemente des Förderns und Forderns so lange miteinander verglichen und die Typen rekonstruiert, bis die den Typen zugeordneten Fälle in einem Großteil der Merkmale ähnlich waren, wie dies Udo Kelle und Susann Kluge für eine gelungene Typenkonstruktion im Allgemeinen ausweisen (Kelle/Kluge 2010a: 112). Die untersuchten Analysekategorien wurden aus den theoretischen Vorannahmen abgeleitet und motiviert (Kapitel 5.5.2), um die Untersuchungsergebnisse zu kontextualisieren. Diese Typen zeichnen sich durch spezifische Aussagen in den Deutungsmustern und Maßnahmengestaltungen aus. Die vorgefundenen institutionenspezifischen und sozialen Funktionen werden anschließend weiter abstrahiert und die Ebene der sozialstaatlichen Funktionen von Maßnahmen Sozialer Aktivierung analysiert (Kapitel 5.5.3). Insgesamt wird mittels der WDA, die Formation des Aktivierungsdiskurses auf der Ebene von anwendenden Institutionen rekonstruiert (Bedeutungsproduktion). Relevant sind hier diskursive Praktiken der SGB-II- und Maßnahmenträger, die auf Aktivierung von erwerbslosen Leistungsempfangenden zielen, nicht die Deutungen der Arbeitslosen selbst. ${ }^{19}$

Die WDA wird genutzt, da sie die klassische Diskurstheorie Foucaults mit dem interpretativen Paradigma und dem Sozialkonstruktivismus verbindet. Damit stellt sie ein Analyseinstrumentarium zur Verfügung, welches erlaubt, die

19 Dennoch ist bei der Wissenssoziologischen Diskursanalyse methodologisch ein Akteurskonzept in die Diskurstheorie integriert (im Gegensatz zur Diskurstheorie nach Foucault), das soziale AkteurInnen als diskursiv konstituierte, regelinterpretierend Handelnde versteht (Keller 2008: 11). 
Handlungsebene der AkteurInnen der Arbeitsverwaltung mit den sie rahmenden Strukturen des politisch-rechtlichen Aktivierungsdiskurses in Beziehung zu setzen. Mit Peter L. Berger und Thomas Luckmann wird von einer gesellschaftlichen Wirklichkeitskonstruktion ausgegangen - einer Sinnwelt, die in Prozessen interaktiver und kommunikativer Objektivierung entsteht. Ein subjektiver Wissensvorrat wird durch die Trias der Externalisierung, Objektivierung und Internalisierung mit einem objektiven Wissensvorrat verklammert: ${ }^{20} \gg$ Gesellschaft ist ein menschliches Produkt. Gesellschaft ist eine objektive Wirklichkeit. Der Mensch ist ein gesellschaftliches Produkt.«(Berger/Luckmann 2004: 65) Berger und Luckmann beziehen sich dabei auf Alfred Schütz, der die soziale Typik individueller Bewusstseinsinhalte und Wissensstrukturen der alltäglichen Lebenswelt herausstellt und den Symbolischen Interaktionismus, der davon ausgeht, dass Bedeutung im symbolisch vermittelten Prozess der Interaktion hervorgebracht wird: Ergebnis ist »eine Theorie der sozialen Konstruktion von Deutungsund Handlungswissen, das gesellschaftlich institutionalisiert und in Sozialisationsprozessen an Individuen vermittelt wird.« (Keller 2006: 118) Die bereits angeführten »Deutungsmuster« bezeichnen dann »grundlegende bedeutungsgenerierende Schemata, die durch Diskurse verbreitet werden und nahe legen, worum es sich bei einem Phänomen handelt.« (Keller 2008: 243) Deutungsmuster von Aktivierung und Integrationsformen, in die integriert wird, werden zunächst recht weit gefasst. So wird mit Herbert Blumers sensitizing concepts von der Vagheit theoretischer Konzepte der Sozialwissenschaften ausgegangen (1940, 1954) und die Begriffe »Aktivierung« sowie »Integrationsform« zunächst als offenes Konzept verwendet. In der Empirie werden diese durch die soziale Lebenspraxis konkretisiert, indem die sozialen Handlungskontexte mit ihren signifikanten Gesten der AkteurInnen zum Vorschein kommen, die dem Begriff ihre Bedeutung verleihen. Die letztendlichen Begriffsbestimmungen von Aktivierung und Integration über Tätigkeit werden dem Aktivierungsdiskurs als Vergleichsfolie sowie der Akteurslandschaft entnommen. Dabei steht nicht die Frage im Vordergrund, was Aktivierung und Integration sind, sondern es wird gefragt, wie Aktivierung und Integration sind und welchen Grund es gibt, sich aktivieren zu lassen und eine Tätigkeit aufzunehmen (etwa in Erwerb oder einer beschäftigungsfördernden Maßnahme). Wissenschaftstheoretisch gesehen geht es also nicht darum, andere Begriffe den Aktivierungs- und Integrationsbegriffen zuzuordnen, sondern diese anhand von Erscheinungen der Wirklichkeit, mit denen sie

20 Voraussetzung der Interaktion bilden gewisse gemeinsame Grundelemente des Wissens: Grundstrukturen der Lebenswelt und kulturspezifische Wissensformen. 
korrespondieren, und den damit verbundenen Eigenschaften zu identifizieren. Um Institutionen verstehen zu können, muss das vortheoretische Wissen (Rezeptwissen nach Alfred Schütz) über diese analysiert werden. Denn Institutionen als Handlungsgefüge, die partikulare, subjektive Handlungsentwürfe via sozial objektivierten Bedeutungen des Wissens (»Wissensvorrat «) transzendieren, fungieren weiter als Indikatoren des gesellschaftlichen Relevanzsystems, da sie typische Lösungen für wiederkehrende gesellschaftliche Handlungsprobleme darstellen (Knoblauch 2005: 159).

Zusammengefasst wird die Wissenssoziologische Diskursanalyse gewählt, da diese der diskursiven Konstruktion sowie dem Wissensbegriff Rechnung trägt. In den Fokus gelangt dadurch eine Praxis von Aktivierung, die sich in den Institutionen der Arbeitsverwaltung etabliert. Im Gefolge verändern die AkteurInnen die dispositiven Elemente des Aktivierungsdiskurses. Anhand der Wissenssoziologischen Diskursanalyse können sowohl das kommunikative Geschehen als auch Praktiken analysiert werden (Keller 2013a: 91), die in der Arbeitsverwaltung vorherrschen.

\subsubsection{Grounded Theory als Orientierung für die Erhebung}

Das Sample umfasst fünfundvierzig leitfadengestützte Experteninterviews aus elf kontrastierenden Städten und Regionen des Bundesgebiets. ${ }^{21}$ Geachtet wurde auf eine breite regionale Streuung gewichtiger Parameter (etwa Stadt-Land, topografisch, sozial-ökonomisch, auf Basis der Arbeitslosenquote und der Organisationsformen der SGB-II-Aufgabenwahrnehmung). Im Sample befinden sich zehn Jobcenter, die aus einer Arbeitsgemeinschaft von Arbeitsagentur und Kommune hervorgegangen sind und zwei Einrichtungen aus zugelassenen kommunalen Trägern (zkT, im Feld auch Optionskommune genannt). ${ }^{22}$

Die leitfadengestützten Experteninterviews dauerten durchschnittlich $90 \mathrm{Mi}-$ nuten. Die Spanne reicht dabei von 45minütigen bis dreistündigen Gesprächen. Überwiegend wurden die Experteninterviews über Entstehung und Gestaltung einzelner Maßnahmen auf verschiedenen Entscheidungsebenen geführt, um möglichst umfassend Sachinformationen, Motivationen und erwünschte Effekte in divergierenden Funktionsstellen in die Untersuchung einbinden zu können.

21 Eine anonymisierte Auflistung der interviewten Personen und Organisationen befindet sich im Anhang.

22 Die Arbeitsgemeinschaft in Regio06 und das Jobcenter in Regio13. 
»Sie informieren über die Insider-Erfahrungen spezifischer Status- und Interessengruppen und eröffnen den Zugriff auf implizite Regeln, die an der Schnittstelle zwischen makro- und mikrosoziologischer Analyse zu verorten sind.« (Liebold/Trinczek 2009: 53) Daher liegt der Fokus auf der Rekonstruktion der Entscheidungsmaximen und Deutungsmuster zu Aktivierung und Integration in den spezifischen Funktionsbereichen, wie dies Bogner und Menz für Experteninterviews vorschlagen (Bogner/Menz 2009: 70).

Im dreizehnmonatigen Erhebungszeitraum wurden 44 von insgesamt 45 in Protokollen erfassten Experteninterviews von der Autorin geführt und mit Einbeziehung teilweise zur Verfügung gestellter Materialien zu den Maßnahmen protokolliert. Die vorausgegangene Fallauswahl orientierte sich an der Methode des theoretischen Samplings, bei dem die zu vergleichenden Maßnahmen nach ihrer Relevanz für das Thema ausgewählt wurden (Glaser/Strauss 2005). Maßnahmen Sozialer Aktivierung wurden recherchiert durch Anfragen bei SGB-IITrägern sowie im BA-Intranet und auf Websites von Maßnahmenträgern. Wenn die skizzierten Maßnahmen als sozialintegrativ und persönlichkeitsfördernd angelegt erschienen, wurden Interviews mit in diesem Kontext stehenden SGB-IIund Maßnahmenträger-Fachkräften durchgeführt. Dieses Auswahl-Verfahren ermöglichte die Erfassung unterschiedlicher Fallanalysen der Maßnahmen und eine hinreichend dichte Kontrastierung divergierender Maßnahmenformen. Teils wurden die Interviews direkt durch die SGB-II-Träger und teils durch die Autorin organisiert. Ergänzt wurde das Sample durch zwei Telefoninterviews und ein Memo über eine weitere Maßnahme. Neben den Institutionen der Beschäftigungsförderung wurden andere beratende Institutionen zu ihren Erfahrungen mit arbeitsmarktfernen Langzeitarbeitslosen und niederschwelligen Angeboten für diese Klientel befragt wie sozialpsychiatrische Dienste oder eine christliche Beratungsstelle,$^{23}$ um dem Prinzip der Minimierung und Maximierung von Unterschieden (Glaser/Strauss 2005: 62ff.) zu folgen. Grundannahme ist dabei, dass die Erhöhung der Datenvarianz durch vielfältige Datenquellen das analytischtheoretische Verständnis des Untersuchungsgegenstandes erweitert.

Der Leitfaden für die Interviews mit Expertinnen und Experten wurde an die Institutionsform sowie im Laufe der Erhebung an neue Erkenntnisse und Fragestellungen angepasst. In den ersten sechs Interviews diente eine Rahmenerzäh-

23 Weitere Ränder des Themenfeldes zur Kontrastierung waren ein Psychologischer Dienst eines SGB II-Trägers (Telefoninterview02Regio02) oder die ehrenamtliche Unterstützung einer Nachhilfeschule und eines Boxvereins (Telefoninterview01Regio03). 
lung dazu, den Interviewten verständlich zu machen, welche Form von Maßnahmen die Autorin interessierte. Aus dieser Erhebungsphase resultierte der den Interviews entstammende in vivo code »niederschwellige Maßnahmen «, der Maßnahmen Sozialer Aktivierung beschreibt. Daher fiel ab dem siebten Interview die Rahmenerzählung weg. Ferner wurde der Leitfaden nicht akribisch >abgearbeitet`, sondern auf den Gesprächsverlauf bezogen. Die Gespräche wurden aufgezeichnet und protokolliert. Für die Analyse waren die Protokolle die zentrale Datenquelle, wobei die Audiodateien und teilweise von den GesprächspartnerInnen ausgehändigte Dokumente in dem Analyseprozess ebenso Eingang fanden. Die größtmögliche Offenheit (Brüsemeister 2000: 197) für Phänomene in der Beschäftigungsförderung, die nur mittelbar auf den Arbeitsmarkt zielen, der Persönlichkeitsentwicklung und Förderung von über reine Bildungsangebote hinausweisenden Fähigkeiten der Teilnehmenden diente, war gerade mit einer Orientierung an der Grounded Theory möglich. Insbesondere, da zu Studienbeginn noch keine Begrifflichkeiten der AkteurInnen für die gesuchten Maßnahmen bekannt waren, denn die Zugriffsweise der AkteurInnen ist meist über die rechtliche Realisierungsform von Maßnahmen bestimmt (zum Beispiel Arbeitsgelegenheit). Weiter sollten die empirischen Erkenntnisse nicht über eine Nachfrage nach Maßnahmen, die auf Langzeitarbeitslose mit mehreren Vermittlungshemmnissen zielen, auf den SGB-II-Bereich beschränkt werden. Entgegen der Vorstellung eines rein induktiven Vorgehens ${ }^{24}$ wurde die theoretische Sensibilität genutzt, als »Fähigkeit des Forschers [...] über empirisch gegebenes Material in theoretischen Begriffen zu reflektieren« (Kelle 1994: 305), indem die forschungsleitenden Annahmen (Kapitel 3.3) während der Erhebung und der Analyse methodisch kontrolliert rückbezogen wurden. Zudem wurde die Methode der Komparation (Glaser/Strauss 2005: 32) verwendet, indem systematisch Vergleiche im Auswahlverfahren und der Auswertung des Datenmaterials vorgenommen wurden. Als Parameter dienten etwa Stadt-Land-Lage, sozial-ökono-

24 Die Annahme, dass Forschende ohne Vorannahmen in das Feld gehen, um die vorzufindenden Daten nicht in ein bestehendes Theorienraster einzubetten, wie dies Glaser und Strauss (2005) nahelegen, sondern dem zu untersuchenden Gegenstand gegenüber offen zu sein, soll gewährleisten, dass Sinnzuschreibungen der Handelnden und nicht die Deutung der Forschenden wiedergegeben werden. Dies ist Werner Meinefeld zufolge ein hehres Ziel, dass jedoch aus erkenntnistheoretischer Sicht kaum realisierbar ist (2005: 271). Weitere methodologische Einschränkungen werden der Grounded Theory insbesondere hinsichtlich des induktiven Vorgehens durch Udo Kelle (1994) und Jörg Strübing (2008) zugesprochen. 
mische Situation, Arbeitslosenquote und die Organisationsform der SGB-IIAufgabenwahrnehmung sowie die insbesondere für die Untersuchung der Funktionen bedeutsamen Kategorien Maßnahmenziel, Sicht der AkteurInnen, Elemente des Förderns und Forderns, Integrations- und Aktivierungsform. Die Methode des ständigen Vergleichens

»knüpft implizit an die von Everett C. Hughes propagierte Kontrastierung divergierender Daten an, bei der mit Hilfe der systematischen Befragung der Daten auf Unterschiede und Ähnlichkeiten sowohl Spezifika einzelner Phänomene als auch mehrere Phänomene übergreifende Homologien erarbeitet werden.« (Strübing 2008: 18)

Zusammengefasst orientierte sich die von der Autorin durchgeführte Erhebung von qualitativen Experteninterviews an der Grounded Theory, indem die Expertinnen und Experten nach der Methode des theoretical Samplings und dem Prinzip der Minimierung und Maximierung von Unterschieden ausgewählt wurden. Bereits während der Erhebung wurden systematische Vergleiche vorgenommen sowie die Interviews ausgewertet, um auf Basis der neuen Erkenntnisse weitere Fallauswahlen durchzuführen.

\subsection{Geltungsbereich der Studie}

Die Beschäftigungsförderung ist ein weites und teilweise bereits gut beforschtes Feld, auf welches die vorgelegte Studie nicht in seiner Gänze eingehen kann. Vielmehr konzentriert sich diese auf einen kaum erforschten spezifischen Teilbereich: die auf Persönlichkeitsförderung und soziale Integration ausgelegte Beschäftigungsförderung, die nur in langfristiger Perspektive als arbeitsmarktbezogene Förderung angesehen werden kann - Maßnahmen Sozialer Aktivierung. Der Geltungsbereich der nachfolgend getroffenen Aussagen zeichnet sich durch folgende Grenzziehungen aus:

1.) Den Interviews kommt eine heuristische Bedeutung zu, eine repräsentative empirische Evidenz über die Maßnahmen Sozialer Aktivierung liefern sie nicht. Vielmehr sollen Handlungsmuster und daraus entwickelbare theoretische Konzepte genügend differenziert sein, um das zu untersuchende Phänomen dicht beschreiben und ausreichend erklären zu können (Brüsemeister 2000). Denn durch das explorative Design der Interviews sollte die Breite und Vielschichtigkeit der sozialen Aktivierung, der mit ihr verbundenen Deutungsmuster und ihre Entstehung aufgezeigt werden. Ferner könnten 
Verlaufsdaten über mehrere Maßnahmen hinweg tiefergehende Auskünfte über die Genese von sozial aktivierenden Maßnahmenketten respektive an Maßnahmen Sozialer Aktivierung anschließende beschäftigungsfördernde Instrumente bieten.

2.) Betrachtet wird in der vorliegenden Studie das Verhältnis zwischen dem Aktivierungsdiskurs einerseits und den Deutungsmustern von Aktivierung und sozialen Integrationsformen andererseits. Die Subjektkonstitution wird über die organisationale Ebene der AkteurInnen, die mit dem Entstehungs- und Durchführungsprozess von Maßnahmen Sozialer Aktivierung betraut sind, analysiert. Unberücksichtigt bleibt dagegen die individuelle Perspektive der Maßnahmenteilnehmenden. Weder ihre individuellen Deutungsmuster noch ihre Wahrnehmung der Funktionen von Maßnahmen Sozialer Aktivierung könnten mit dem für die Studie erhobenen Material beforscht werden.

3.) Die vorgenommene Wissenssoziologische Diskursanalyse ermöglicht es, gouvernementale Interventionen innerhalb der untersuchten Wahrheitsregime zu analysieren, wobei keinerlei allgemeine Aussagen über die Gültigkeit oder Ungültigkeit des Wissens getroffen werden können. Es werden die Produktionen von Wahrheit in administrativen Prozeduren, diskursiven Operationen und institutionelle Legitimationen als Wissenspolitik offengelegt. Insofern bezieht sich die Problemdefinition auf den Aktivierungsdiskurs selbst und die in ihm vorgenommenen Interventionen.

Begrenzt durch die genannten Einschränkungen, vermag diese Studie das Entstehen und die Funktionen von Maßnahmen Sozialer Aktivierung zu explorieren und rekonstruieren. 


\section{Aktivierungsdiskurs: Wandel der Arbeitsmarktpolitik}

Die »Gesetze für Moderne Dienstleistungen am Arbeitsmarkt« I bis IV (besser bekannt als »Hartz-Gesetze«) verändern 2004/2005 die deutsche Arbeitsmarktpolitik grundlegend. Stand zuvor die aktive staatliche Arbeitsmarktpolitik im Vordergrund, verlagerte sich der Schwerpunkt hin zur aktivierenden Arbeitsmarktpolitik. Zentral ist in der letzteren die Koppelung von Rechten wie der sozialstaatlichen Leistungsgewährung an »soziale Pflichten« der Leistungsbeziehenden. Der bisher überwiegend alimentierende Staat tritt unter dem Imperativ der Aktivierung nicht mehr ohne »Gegenleistung« auf, beziehungsweise fordert diese verstärkt von Sozialleistungsempfangenden ein. Zwar sind Mitwirkungspflichten für Sozialversicherungssysteme üblicherweise konstitutiv, allerdings haben sich Umfang und Gewicht der Mitwirkung stark verändert. So wird den Erwerbslosen mehr Eigenverantwortung zugesprochen und abverlangt. Zudem werden strengere Kriterien der Bedürftigkeitsprüfung und schärfere Zumutbarkeitsregelungen angelegt. ${ }^{1}$ Demgegenüber steht den erwerbslosen Leistungsempfangenden kein funktionales Äquivalent zu den Druckmitteln der SGB-IITräger-Beschäftigten zur Verfügung.

Da sich die tägliche Praxis der Arbeitsverwaltung immer auch in und vor dem Hintergrund eines in Bezug auf sie geführten politisch-rechtlichen Diskur$\operatorname{ses}^{2}$ vollzieht, ist insbesondere das Instrument der Wissenssoziologischen Diskursanalyse geeignet, Strukturprinzipien und Deutungsmuster in der Arbeitsverwaltung zu erklären. Für die Diskursanalyse relevante Quellen bilden 1.) Ge-

1 Diese Steuerungsänderung dokumentiert sich auch in dem markanten Vermögensrückgang von Arbeitslosen (Grabka/Westermeier 2014).

2 Zur allgemeinen Diskursdefinition vergleiche Kapitel 3.3.2 und 3.4.1. 
setzgebungen und Gesetze vorbereitende Veröffentlichungen, 2.) Berichte sowie Informationen für die SGB-II-Leistungsbeziehenden respektive die Öffentlichkeit von BA, BMWA, BRH, SGB-II-Trägern und 3.) innerinstitutionelle Texte, die Handlungsempfehlungen und Geschäftsanweisungen (sogenannte HEGAs) für die Fachkräfte bieten. Der Schwerpunkt liegt auf dem SGB II (Deutscher Bundestag 2013b) und dem Bericht der Hartz-Kommission, da die Maßnahmen Sozialer Aktivierung vor allem im SGB-II-Bereich eingesetzt werden. ${ }^{3}$

Tabelle 1: Übersicht über die Quellen der Diskursanalyse

\begin{tabular}{|l|l|}
\hline Textform & Anzahl \\
\hline Gesetzgebungen und vorbereitende Veröffentlichungen & 10 \\
\hline $\begin{array}{l}\text { Berichte und Informationen für SGB-II-Leistungsbeziehende } \\
\text { sowie die Öffentlichkeit }\end{array}$ & 18 \\
\hline Innerinstitutionelle Texte (HEGAs, Arbeitshilfen) & 8 \\
\hline
\end{tabular}

Quelle: Eigene Darstellung

Die Diskusanalyse erstreckt sich auf folgende Aspekte: Erstens die Darstellung des aktivierenden Staates mit seiner Zielsetzung und der gesetzlichen Verankerung, die Ausgangspunkt für die Beschäftigungsförderung und die Maßnahmen Sozialer Aktivierung sind (Kapitel 4.1), zweitens die Sicht der AkteurInnen der Beschäftigungsförderung auf die erwerbslosen SGB-II-Leistungsbeziehenden als zu aktivierende AdressatInnen (Kapitel 4.2), drittens die arbeitsmarktlich-ökonomische Strategie im Hinblick auf die wirtschaftliche Komponente der Erwerbsintegration (Kapitel 4.3). Diese drei Aspekte haben im Sinne des Aktivierungsdiskurses eine beträchtliche Wirkung auf die Integration in Erwerbstätigkeit und, dadurch vermittelt, die Funktion der sozialen Teilhabe in der Gesellschaft (Kapitel 4.4). Im Aktivierungsdiskurs lassen sich jedoch auch hybride Momente finden, in die sich widersprechende Zielsetzungen eingegangen sind (Kapitel 4.5). Dies wird an den Beispielen der Bedarfsgemeinschaft und Familie sowie der Beziehung von aktivierender Arbeitsmarktpolitik und dem subventionierten Ar-

3 Die Analyse entwickelt zentrale Gedanken einer vorausgegangenen Aktivierungsdiskursanalyse weiter (Freier 2008). 
beitsmarkt behandelt. Darauf aufbauend werden die Implikationen für die SGBII-Träger analysiert (Kapitel 4.6).

\subsection{Der aKtivierende StaAt}

Im Rahmen des Bündnisses für Arbeit (Ausbildung und Wettbewerbsfähigkeit), dem von der Bundesregierung initiierten Zusammenschluss mit Gewerkschaften und Arbeitgeberverbänden, entstand das 2001 verabschiedete »Job-AQTIVGesetz« (Deutscher Bundestag 2001), das als Vorläufer der Gesetzgebung für Moderne Dienstleistungen am Arbeitsmarkt gilt (Walwei 2009, Hassel/Schiller 2010). Mit ihm wurde eine Reform des Sozialstaates und Arbeitsmarktes eingeleitet, die aus Sicht ihrer ProtagonistInnen auf die Minderung der Arbeitslosigkeit zielt. Der für den aktivierenden Staat zentrale Leitgedanke »Fördern und Fordern« stammt aus diesem Papier, war allerdings zugeschnitten auf das SGB III (Deutscher Bundestag 2013a), also die arbeitsmarktnahe Klientel. 2002 legte die Kommission »Moderne Dienstleistungen am Arbeitsmarkt« (HartzKommission 2002) Empfehlungen zum Umbau des Arbeitsmarktes vor, die 2003 in die Agenda 2010 eingingen und größtenteils durch die »Gesetze für Moderne Dienstleistungen am Arbeitsmarkt« I bis IV umgesetzt wurden (»Hartz-Gesetze «). ${ }^{4}$ Insbesondere letztere veränderten zum Jahreswechsel 2004/2005 den Arbeitsmarkt grundlegend (Deutscher Bundestag 2003b). Stand im Job-AQTIVGesetz noch eine aktive staatliche Arbeitsmarktpolitik im Vordergrund, verlagerte sich mit den Hartz-Gesetzen der Schwerpunkt auf eine aktivierende Arbeitsmarktpolitik. Der aktivierende Staat erweitert die Durchsetzungsmechanismen, um das Primärziel der Aufnahme einer Erwerbstätigkeit (§ 1 Abs. 2 SGB II) zu erreichen und fordert stärker, als der frühere alimentierende Staat, Gegenleistungen ein. Eigenverantwortung (»Hilfe zur Selbsthilfe« $§ 1$ Abs. 1 SGB II) wird priorisiert und strengere Bedürftigkeitskriterien bei geweiteten Zumutbarkeitsregelungen angelegt.

Der politisch-rechtliche Diskurs von aktivierendem Staat und aktivierender Arbeitsmarktpolitik in Deutschland steht dabei in einem europaweiten Kontext, angelehnt an die britische Work first-Politik. Der sogenannte Luxemburger Prozess brachte die Europäische Beschäftigungsstrategie hervor, die nationale Reaktionen zur Bekämpfung der Jugend- und Langzeitarbeitslosigkeit vorsieht, bei

4 Siehe (Deutscher Bundestag 2002a), (Deutscher Bundestag 2002b), (Deutscher Bundestag 2003a), (Deutscher Bundestag 2003b). 
denen »aktiven Maßnahmen zur beruflichen Eingliederung konsequent der Vorrang vor passiven Unterstützungsmaßnahmen gegeben wird.« (Rat der EU 1997: 4) Laut BA beschreitet Deutschland mit der Grundsicherung für Arbeitsuchende »neue Wege bei der aktivierenden Arbeitsmarktpolitik « (BA 2006: 87). Zugleich werden wesentliche Elemente europäischer Beschäftigungsstrategie umgesetzt, indem es

»die in ihren Leitlinien neben finanziellen Anreizmechanismen (〉Arbeit lohnend machen`) und dem Zugang zu Aktivierungsmaßnahmen (〉Fördern $)$ seit 2003 auch die Überprüfung von Lohnersatzquoten und die Dauer des Leistungsbezugs (〉Fordern`) hinsichtlich einer effektiven Arbeitssuche von den Mitgliedsstaaten einfordert.«(BA 2006: 88)

Grundannahme des Diskurses ist, dass die Ursache der Massenarbeitslosigkeit nicht primär im Mangel an Arbeitsplätzen auf Grund konjunktureller Rahmendaten, sondern an der defizitären Qualifikation und Motivation der Erwerbslosen liegt. Hauptziel ist Integration möglichst vieler erwerbsfähiger Personen in den ersten Arbeitsmarkt, somit wird die »Bekämpfung der Arbeitslosigkeit« zur vorrangigen Aufgabe der Gesellschaft erklärt (Hartz-Kommission 2002: 37). So wird die Anzahl der Erwerbsaufnahmen in messbaren Integrationsquoten über jährliche Zielvereinbarungen für die SGB-II-Träger festgelegt.

»Die Grundsicherung [...] soll die Eigenverantwortung von erwerbsfähigen Hilfebedürftigen und Personen, die mit ihnen in einer Bedarfsgemeinschaft leben, stärken und dazu beitragen, dass sie ihren Lebensunterhalt unabhängig von der Grundsicherung aus eigenen Mitteln und Kräften bestreiten können.«(§ 1 Abs. 1 SGB II)

Die Grundsicherung soll durch das Dienstleistungs- und Förderangebot (zudem durch Sanktionen) das zu aktivierende Individuum unterstützten (Hartz-Kommission 2002: 19). Kernpunkte im Wirken der den Diskurs tragenden Institutionen sollen hier kurze Übergangsphasen und schnelle Vermittlung in Tätigkeiten sein, wobei die zugrunde liegende Annahme ist, dass jeder Mensch sich selbst in den ersten Arbeitsmarkt integrieren könne, wenn Preis (Lohn) und Leistung (Qualifikation) angemessen seien.

Arbeitslosen- und Sozialhilfe wurden in weiten Teilen zur Grundsicherung für Arbeitsuchende zusammengeführt. Dies beendete das »jahrzehntelange ineffiziente Nebeneinander zweier steuerfinanzierter und bedarfsorientierter Transfersysteme.« (Hüther/Scharnagel 2005: 26) Drei Leistungen stehen nach der Arbeitsmarktreform zur Verfügung: 1.) das Sozialgeld (es steht ähnlich der früheren Sozialhilfe nichterwerbsfähigen Personen zu und wird von den Sozialämtern 
kommunal verwaltet), 2.) das von der BA verwaltete Arbeitslosengeld (Alg) ${ }^{5}$ als »beitragsfinanzierte originäre Versicherungsleistung « und 3.) das Arbeitslosengeld II (Alg II) als steuerfinanzierte bedürftigkeitsabhängige Leistung zur Sicherung des Lebensunterhalts der arbeitslosen erwerbsfähigen Personen. Letzteres kann in Anspruch genommen werden, wenn die Bedingungen für das Alg nicht erfüllt sind beziehungsweise die Bezugsdauer für das Alg abgelaufen ist. Die Grundsicherung für Arbeitsuchende ist im SGB II verankert. Im Gegensatz zur früheren Arbeitslosenhilfe ist die Höhe der Leistung unabhängig vom zuletzt erzielten durchschnittlichen Nettoeinkommen, sondern der Sozialhilfe angeglichen - also ein Übergang von der individuellen, versicherungsorientierten zur kategorialen Versorgung. Die Regelleistung zur Sicherung des Lebensunterhalts berücksichtigt neben Ernährung, Kleidung, Körperpflege etc. »in vertretbarem Umfang $[\ldots]$ Beziehungen zur Umwelt und eine Teilnahme am kulturellen Leben.« (§ 20 Abs. 1 SGB II) Gleichzeitig soll durch die Bezeichnung Grundsicherung zum Ausdruck gebracht werden, dass es sich um eine »Absicherung des Mindestbedarfes [...], eine Sicherung des Existenzminimums, das zum Leben notwendig ist " (BA 2008a: 8) handelt. Auch werden Personen im SGB-II-Bezug systematischer in die Förderpolitik einbezogen, als in der früheren Sozialhilfe (§§ 14-16g SGB II) und erhalten in klassischer Arbeitsmarktpolitik nicht enthaltene soziale Integrationsleistungen wie die psychosoziale Betreuung (§16a SGB II) (Bieback 2009: 189f.). Doch trotz der Separierung in das Arbeitslosengeld II im SGB II und dem Arbeitslosengeld im SGB III obliegt ein Großteil der Beschäftigungsförderung des SGB II den Zielsetzungen des SGB III und infolgedessen der Priorität der Vermittlung in Erwerbstätigkeit.

Zusammengefasst führten die Hartz-Gesetze zu einer Wandlung vom alimentierenden hin zum aktivierenden Staat, indem für erwerbsfähige Hilfebedürftige eine Grundsicherung zur Sicherung des Existenzminimums besteht, jedoch die sozialstaatlichen Leistungen an eine Gegenleistung der Erwerbslosen gebunden sind. Ferner werden insbesondere individuelle Faktoren der Personen für den Status der Erwerbslosigkeit verantwortlich gemacht.

5 Das Arbeitslosengeld wird auch Arbeitslosengeld I oder ALG I genannt. 


\section{2 "MENSCHEN IN ARBEIT BRINGEN « : ${ }^{6}$ SICHT AUF DIE LEISTUNGSBEZIEHENDEN}

Der Aktivierungsdiskurs stützt sich auf die grundlegende These, dass Aktivierung von Individuen im Leistungsbezug zur Aufnahme von Erwerbstätigkeit führt. Damit nehmen die im Diskurs auftretenden Einschätzungen und Erwartungen an die Leistungsbeziehenden, die sogenannten Kundinnen und Kunden, einen zentralen Bestandteil ein. Auf die Darstellung des Paradigmenwechsels, den das Verständnis von den Leistungsbeziehenden als KundInnen auszeichnet (Kapitel 4.2.1), folgen die Implikationen der Sichtbarkeitskampagne eines ausgeweiteten Personenkreises von Aktivierbaren (Kapitel 4.2.2) sowie die Klassifizierung in Erwerbsfähige und Nicht-Erwerbsfähige (Kapitel 4.2.3).

\subsubsection{Arbeitsmarktdienstleistungen für Kundinnen und Kunden}

Die SGB-II-Leistungsbeziehenden, die als sozialstaatliche Versicherungsleistung Leistungen für ihren Lebensunterhalt ebenso beziehen wie Arbeitsmarktdienstleistungen, werden im Sinne des New Public Management als Kundinnen und Kunden bezeichnet und verstanden. Die Leistungen richten sich an Staatsbürgerinnen und -bürger, die spezifische Voraussetzungen erfüllen müssen ${ }^{7}$ und genauso über die sozialstaatlichen Leistungen sanktioniert werden können, wenn etwa eine aktive Mitwirkung zur Erwerbsaufnahme während des Leistungsbezuges nicht eingehalten wird. So führt der Kundenbegriff weitreichende Implikationen mit sich:

»Machen Kunden von den Angeboten Gebrauch und werden in diesem Sinne Eigenaktivitäten ausgelöst, so können Arbeitslose beim JobCenter Ansprüche auf soziale und materielle Sicherheit durch Geldleistungen einlösen.«(BMAS 2002: 97)

Die Erwerbslosen sollen eigeninitiativ und eigenverantwortlich auf ihre Integration in den ersten Arbeitsmarkt hinwirken. Im Rahmen eines Tausches können sie für die erbrachte Vorleistung sozialstaatliche Unterstützung gewissermaßen

6 Titel und Slogan einer Broschüre des BMWA zum Vierten Gesetz für moderne Dienstleistungen am Arbeitsmarkt: »Hartz IV. Menschen in Arbeit bringen« (BMWA (ehemaliges) 2005a).

7 Vergleiche Kapitel 4.2.2 »Arbeitslosigkeit `bekommt ein Gesicht««: Aktivieren eines erweiterten Personenkreises. 
einlösen. Die Vorstellung vom menschlichen Sein entspricht einem aktiven Wesen, das sich durch Arbeitsverrichtung selbstverwirklichen und soziale Kontakte herstellen kann. Ferner ist das Individuum frei, mündig und entscheidungsfähig (Hartz-Kommission 2002: 97). Das eigeninitiative Handeln wird jedoch staatlicherseits auf seine Erwerbsaufnahme-Orientierung hin geprüft. So werden den Kundinnen und Kunden Angebote zur Erwerbsintegration unterbreitet, die von diesen genutzt oder ungenutzt bleiben können. Doch nur, wenn die Angebote genutzt wurden respektive nachweisliche Bemühungen zur Erwerbsaufnahme bestehen, wird eine umfassende sozialstaatliche Unterstützung gewährt. Dieser Prozess soll durch Beratung und weitere Arbeitsmarktdienstleistungen unterstützt werden. Zwar erhalten auch sanktionierte Personen eine Grundversorgung, doch die Logik der sozialstaatlichen Unterstützung folgt einer Verantwortungsübertragung der sogenannten Responsibilisierung und nicht einer Bedürftigkeitsprüfung. Betriebswirtschaftliche Steuerelemente und ein neues Verständnis der Verwaltung, ${ }^{8}$ führen nicht nur zu einer Vermarktlichung der Arbeitsverwaltung, auch die Sichtweise auf den zu beratenden Erwerbslosen hat sich top-down verändert: In der Sicht des Diskurses sind Erwerbslose nun Kundinnen und Kunden, die Arbeitsmarktdienstleistungen in Anspruch nehmen. Dies überantwortet zwar auch der Verwaltung die Maßgabe, sich an den Anliegen und Bedürfnissen der >Dienstleistungsnehmer` zu orientieren. Allerdings werden die Erwerbslosen zugleich auch in ihrer Subjektivität angerufen, wie Bröckling dies für das unternehmerische Selbst darstellt (Bröckling 2007, 2000), indem sie selbstständig als KundInnen in der Arbeitsmarktverwaltung operieren sollen. Entsprechend haben Arbeitslose

»die Pflicht zur Mitwirkung und zur Annahme eines zumutbaren Stellenangebotes (Annahmepflicht). Grundsätzlich wird dies durch die Anwendung einer neuen Sperrzeitregelung gewährleistet, dass die Leistung nur so lange ohne Kürzung bzw. überhaupt weitergezahlt wird, wie sich der Arbeitslose aktiv um Arbeit bemüht.« (Hartz-Kommission 2002: 94)

Garantiert das System der Vollerwerbstätigkeit im Diskurs den Wohlstand der Allgemeinheit, besitzt es unmittelbare Konsequenzen für das Individuum, denn dieses kann über die durch Arbeit erwirtschafteten monetären Mittel ein selbstbestimmtes Leben führen und wird mittels Arbeit in die Gesellschaft integriert. Dabei zeichnet sich das Individuum durch eine spezifische »Beschäftigungsfä-

8 Vergleiche Kapitel 4.6 Diskurstragende Institutionen in der Arbeitsverwaltung. 
higkeit« (Hartz-Kommission 2002: 46) aus, vergleichbar mit der »employability« aus dem Workfare-Konzept.

»Mit dem Begriff `Beschäftigungsfähigkeit` werden die Fähigkeiten einer Person beschrieben, auf der Grundlage ihrer fachlichen und Handlungskompetenzen, Wertschöpfungs- und Leistungsfähigkeit ihre Arbeitskraft anbieten zu können und damit in das Erwerbsleben eintreten zu können, ihre Arbeitsstelle zu halten oder, wenn nötig, sich eine neue Erwerbsbeschäftigung zu suchen.« (Blancke et al. 2003: 652 zit. nach Wolf 2006, 1086 Fußnote 17)

Im Aktivierungsdiskurs ist die/der Arbeitslose also durch Wahl- und Handlungsoptionen dazu befähigt, selbst »im Sinne des Integrationszieles« (Hartz-Kommission 2002: 45) tätig zu werden und dabei unter anderem die Dienstleistungen des SGB-II-Trägers zu nutzen. Denn die »neue Qualität der Dienstleistungsbeziehung « (Hartz-Kommission 2002: 46) der Arbeitsagentur ermögliche die individuelle »Wahlfreiheit zwischen unterschiedlichen Optionen wie auch zwischen der grundsätzlichen Annahme des Integrationsangebotes und der Entscheidung für persönliche Alternativen außerhalb des Beschäftigungssystems « (HartzKommission 2002: 46). ${ }^{9}$

Die Aufnahme einer Tätigkeit im ersten Arbeitsmarkt, die mittels der neuen Zumutbarkeit mehr räumliche Mobilität bedeuten kann, liegt im zentralen »Sinne des Integrationszieles« (Hartz-Kommission 2002: 46) und des neuen Dienstleistungsverhältnisses. Die SGB-II-Träger-Fachkraft steht hierbei in der Angebotspflicht und muss eine geeignete Beschäftigung nach Interessen, Fähigkeiten und Entwicklungspotenzial suchen. Die familiäre Situation wird berücksichtigt ( 1 Abs. 1 und $\S 10$ Abs. 1 SGB II), jedoch sind getrennte Haushalte für die Arbeitsaufnahme vorübergehend zumutbar. Menschen ohne Anstellung, die Verantwortung gegenüber betreuungsbedürftigen Personen, Familienangehörigen oder bis zu drei Jahre alte Kinder haben, sind nicht verpflichtet eine Arbeit aufzunehmen. Mit der Dauer der Arbeitslosigkeit (in Abhängigkeit zur familiären Situation) weiten sich die Zumutbarkeitskriterien und damit zum Beispiel Anforderungen an die Mobilität. ${ }^{10}$ Eine Stelle ist zumutbar, wenn die Entlohnung niedriger ist als in der Vergangenheit (Hartz-Kommission 2002: 94ff.).

9 Bei letzterem Weg werden zwar keine Leistungen gezahlt, jedoch bleibt der Versicherungsanspruch erhalten.

10 Nach drei Monaten kann von einem jungen Arbeitslosen ein Umzug verlangt werden, wenn es sich um eine Vollzeitarbeitsstelle handelt. 
Die zu Grunde liegende Annahme des Aktivierungsdiskurses ist, dass der erwerbslose Mensch durch Dienstleistungen der Beschäftigungsförderung zur Eigenaktivität befähigt wird. Für diese Hilfestellung und seine Existenzsicherung soll die/der »Hilfebedürftige« eine Gegenleistung erbringen: er ist verpflichtet den >Schaden` zu mindern, der durch seine Erwerbslosigkeit dem Sozialstaat entsteht. Das Individuum wird im Diskurs als selbstverantwortlich angesehen und mit einer räumlichen und erwerbstätigkeitsbezogenen Flexibilitätsanforderung versehen. ${ }^{11}$ Persönliche Eigenverantwortung wird hier gegenüber einer gesellschaftlich-garantierten Sicherung betont und damit ein über Jahrzehnte geltendes Verständnis abgelöst, wie es in Art.20 I GG seinen verfassungsrechtlichen Ausdruck hat und im früheren BSHG festgelegt war (Wolf 2006: 1082). Mit dem allgemeinen verfassungsrechtlichen Verdikt »Die Würde des Menschen ist unantastbar. Sie zu achten und zu schützen ist Verpflichtung aller staatlichen Gewalt.« (Art.1 I GG) korrespondiert § 1 II des BSHG, dass als Aufgabe der Sozialhilfe definierte, »dem Empfänger der Hilfe die Führung eines Lebens zu ermöglichen, das der Würde des Menschen entspricht. « Dieser Passus ist im aktuellen SGB II nicht mehr enthalten. Der Diskurs hat sich entfernt von einer Orientierung an den Grundbedürfnissen und stellt nun vielmehr die Eigenverantwortung der Individuen in den Vordergrund.

Diese Responsibilisierung im Aktivierungsdiskurs beruht einerseits auf der Annahme, dass vom Sozialstaat geleistete Hilfe die Bedürftigkeit zementiert, weil Eigeninitiative gehemmt wird. Andererseits kann im Umkehrschluss durch eine Verringerung staatlicher Fremdhilfe die Einsicht des Bedürftigen in die Notwendigkeit der Selbsthilfe gefördert werden (Wolf 2006: 1081). Voraussetzung für diese Sichtweise ist die Annahme eines grundsätzlichen Handlungsvermögens, das eigenverantwortliches Agieren der Arbeitslosen zulässt. Als Konsequenz dieser Annahme erfolgt allerdings eine implizite Schuldzuweisung an die Arbeitsuchenden bei Versagen der Eingliederung, da diese als »Verfehlung" gedeutet wird - eventuelle externe Hemmnisse werden so systematisch ausgeblendet. Das Marktrisiko wird auf das Individuum übertragen und nicht als von dem Individuum unverschuldete Härte aufgefasst. Im Aktivierungsdiskurs werden so zwei opponierende Positionen integriert: So steht die Grundannahme des in allen sozialen Lagen autonom und rational agierenden Subjekts, als homo oeconomicus, im Widerspruch zu der Annahme, dass Langzeitarbeitslose durch langjährige Untätigkeit in ihrem psychischen und physischen Befinden gestört seien. Jedoch verdeckt die Annahme des grundsätzlich handlungsfähigen Indivi-

11 Vergleiche Kapitel 4.3 Unternehmerische Elemente der Arbeitsmarktpolitik. 
duums existierende Machtkonstellationen und strukturelle Hemmnisse - und legitimiert Aussagen wie die, dass niemand gezwungen wird eine angebotene Stelle anzunehmen oder an einer integrationsfördernden Maßnahme teilzunehmen (Hartz-Kommission 2002: 97). Die/der Anstellungslose wird nicht als abhängiger »Leistungsempfänger" gesehen, der bei Nichtannahme einer zumutbaren Stelle mit Kürzungen rechnen muss, sondern als unabhängiger »Kunde«, der in freier Entscheidung aus dem Angebot der SGB-II-Träger wählt und sich schließlich über die anschließende Tätigkeit verwirklichen kann. Sogenannte hilfebedürftige Leistungsbeziehende handeln folglich autonom und selbstbestimmt, wenn sie sich `entscheiden` andere Möglichkeiten der Lebenssicherung in Anspruch zu nehmen. Die Subjektposition als homo oeconomicus legitimiert so ein philanthropisches Selbstverständnis der SGB-II-Träger. In Abstraktion vom Machtungleichgewicht begegnen sich KundInnen und Jobcenter-Beschäftigte »auf gleicher Augenhöhe« (Hartz-Kommission 2002: 97). Doch die Handlungsoptionen der leistungsbeziehenden Erwerbslosen sind begrenzter, als dies ein Kundenstatus transportiert, der auf Implikationen des wirtschaftlichen Kontextes rekurriert. Die Vermittlungsberatung ${ }^{12}$ erfolgt in rechtlichen Regelungen, die insbesondere für die SGB-II-Leistungsbeziehenden Sanktionen jenseits der zugelassenen Verhaltensweisen vorsieht und dadurch einen Zwangscharakter herstellt (Hielscher/Ochs 2009). Eine Asymmetrie entwickelt sich hier insbesondere durch das Expertenwissen der Vermittlungsberatung gegenüber dem nachfragenden SGB-II-Leistungsbeziehenden, dass unter anderem durch ein mögliches Spektrum der behandelbaren Themen in der Beratungsinteraktion nicht immer vollständig zielführend im Sinne der SGB-II-Leistungsbeziehenden geteilt wird (Schütz et al. 2011). Ferner können die Logiken der Arbeitsvermittlung die an den beruflichen Interessen der Erwerbslosen ausgerichteten adressatInnenzentrierte Hilfepläne beeinträchtigen und das Selbstbewusstsein der SGB-IILeistungsempfangenden einschränken, indem ihre Hemmnisse fokussiert und ihre »Beschäftigungsfähigkeit« wiederkehrend neu vermessen werden (Kratz 2013a). Weiter sind für die Leistungsbeziehenden durch eine Verkürzung von Planungshorizonten und Erwartungssicherheiten nicht die notwendigen Rahmenbedingungen für Autonomie gegeben, vielmehr wird der Autonomiebegriff von seiner historischen Bedeutung entkoppelt und als Synonym für Eigenverantwortung verwendet (Globisch 2012).

12 Der Begriff Vermittlungsberatung geht auf die Studie von Schütz und KollegInnen zurück (2011). 
Zusammengefasst unterstützt die Annahme von Kundinnen und Kunden eine auf Responsibilisierung aufbauende aktivierende Arbeitsmarktpolitik, abstrahiert jedoch gleichzeitig von strukturellen Rahmenbedingungen für Erwerbslosigkeit sowie von den asymmetrischen Strukturen in der Vermittlungsberatung.

\subsection{2 »Arbeitslosigkeit >bekommt ein Gesicht « $:^{13}$ Aktivieren eines erweiterten Personenkreises}

Mit den einschneidenden Gesetzesveränderungen in der Arbeitsmarktpolitik wird ein breiterer Personenkreis in die Klientel der (regelmäßig) zu aktivierenden überführt, denn die strukturell entscheidende Veränderung ist hier die $\mathrm{Zu}$ sammenführung von Arbeitslosen- und Sozialhilfe zur Grundsicherung für Arbeitsuchende. Über die Transferleistungen werden die direkten AdressatInnen im Aktivierungsdiskurs eingebunden. In der Aktivierungspolitik soll Arbeitslosigkeit gleichsam »ein Gesicht« (Hartz-Kommission 2002: 23) erhalten und die »individuell zuschreibbare Verantwortung " (Hartz-Kommission 2002: 86) der abweichenden wie der funktionierenden gesellschaftlichen Subsysteme und Individuen aus der Anonymität heraustreten.

Der Zugang zu Leistungen der Grundsicherung ${ }^{14}$ für Arbeitsuchende setzt insbesondere ${ }^{15}$ die Erwerbsfähigkeit in folgender Definition voraus: Als erwerbsfähig gilt, wer »unter den üblichen Bedingungen des allgemeinen Arbeitsmarktes mindestens drei Stunden täglich « ( 8 Abs. 1 SGB II) arbeiten kann und nicht durch Krankheit oder Behinderung auf absehbare Zeit dazu außerstande ist. Effekt der so geweiteten Kategorie Erwerbsfähigkeit ist erstens eine verringerte Anzahl an Sozialgeldbeziehenden und zweitens eine Erweiterung der Personenzahl im durch das SGB II geregelten Bezug, der durch die Umsetzungspraktiken der SGB-II-Träger noch verstärkt wurde. Die Praxis der Erwerbsfähigkeitsprüfung monierte 2006 der BRH: Demzufolge wurde in beinahe jedem zehnten Fall die Erwerbsfähigkeit nicht überzeugend festgestellt, etwa weil ernstzunehmende

13 Das Zitat steht in Verbindung mit der Einführung des Fallmanagements, das durch eine intensive Betreuung den einzelnen Menschen und dessen persönliche Lebenslage in den Mittelpunkt stellen soll (Hartz-Kommission 2002: 23).

14 Leistungen nach der Arbeitsmarktreform sind 1.) das Sozialgeld, 2.) das Arbeitslosengeld und 3.) das Arbeitslosengeld II, die sogenannte Grundsicherung (vergleiche Kapitel 4.1 Der aktivierende Staat).

15 Weitere Kriterien sind Alter (15. bis 65. Lebensjahr), Hilfebedürftigkeit und der »gewöhnliche [...] Aufenthalt in der Bundesrepublik« (§ 7 Abs. 1 SGB II). 
Zweifel nicht aufgegriffen wurden und vorliegende ärztliche und psychologische Gutachten anderer Leistungsträger ungenutzt blieben (BRH 2006: 19). Außerdem fordert der BRH eine Erwerbsfähigkeitsprüfung nach drei Monaten Arbeitsunfähigkeit statt nach sechs Monaten.

»Durch die medizinische Minimaldefinition von Erwerbsfähigkeit sind mehr als 90 Prozent aller Sozialhilfeempfänger [im Jahr 2005; CF] als prinzipiell erwerbsfähig eingestuft worden. Der zu aktivierende Personenkreis wurde so - vor dem Hintergrund einer schwierigen Arbeitsmarktlage - >auf einen Schlag^ um fast eine Million ausgedehnt.« (KonleSeidl/Lang 2006: 20)

Promberger et al. konstatieren, dass die Beschäftigungsfähigkeit im Rahmen des Aktivierungsparadigmas insbesondere auf individuelle Komponenten verweist, wohingegen das Konzept der pragmatisch-interaktiven Beschäftigungsfähigkeit erst durch die Betrachtung der betrieblichen und institutionellen Regelungen, der Praktiken von Organisationen und am Arbeitsmarkt, den formalen Eigenschaften des Individuums und seinem Arbeitsvermögen Aufschluss über ein in komplexen Beziehungen und gesellschaftlichen Prozessen am Arbeitsmarkt konkurrierendes Individuum ermöglicht (Promberger/Wenzel/Pfeiffer/Hacket/Hirseland 2008). Darüber hinaus erhalten Personen, die mit erwerbsfähigen Hilfebedürftigen in einer Bedarfsgemeinschaft leben, Leistungen, wenn ihre Hilfebedürftigkeit dadurch beendet oder verringert wird und Eingliederungshemmnisse des erwerbsfähigen Hilfebedürftigen beseitigt oder minimiert werden ( $\$ 7$ Abs. 2 SGB II). Hilfebedürftig ist, wer seinen Lebensunterhalt und den der Bedarfsgemeinschaft nicht aus

»eigenen Kräften und Mitteln, vor allem nicht 1. durch Aufnahme einer zumutbaren Arbeit, 2. aus dem zu berücksichtigenden Einkommen oder Vermögen sichern kann und die erforderliche Hilfe nicht von anderen, insbesondere von Angehörigen oder von Trägern anderer Sozialleistungen erhält.« (§ 5 Abs. 1 SGB II)

Weiter müssen erwerbsfähige Hilfebedürftige nun ihr Vermögen bis zu einem altersabhängigen Freibetrag aufbrauchen, bevor sie Leistungen des Alg II beanspruchen können. Dagegen sah das Modell der Arbeitslosenhilfe vor, dem das Blümsche Gerechtigkeitsmodell zugrunde lag, den Bürger dort abzusichern, wo er stand. Ohne das individuelle Vermögen zu berücksichtigen wurde stabilisiert und Verarmungstendenzen vermindert. So haben Arbeitslose heute ein signifikant geringeres Sach- und Gebrauchsvermögen (Grabka/Westermeier 2014). Ferner haben Kinder und Jugendliche, deren Haushaltsvorstand arbeitslos ist, das 
größte Armutsrisiko (BMAS 2013b). Durch das verminderte Absicherungsniveau wird ergo der finanzielle Druck zur Erwerbsaufnahme erhöht.

Die Ausweitung des von den SGB-II-Träger zu adressierenden Personenkreises geht mit einer gesteigerten Aufmerksamkeit gegenüber Personengruppen einher, die als stärker von Erwerbslosigkeit betroffen gelten: 1.) Frauen, genauer Alleinerziehende, 2.) Schwerbehinderte und 3.) jugendliche und ältere Erwerbsfähige - um möglichst alle erwerbsfähigen Personen in eine Beschäftigung zu überführen. Desgleichen führt die Förderung weiblicher Erwerbsbeteiligung im Zeichen der Chancengleichheit zu einer Erweiterung des arbeitsmarktrelevanten Personenkreises. Daher soll »geschlechtsspezifischen Nachteilen von erwerbsfähigen Hilfebedürftigen entgegengewirkt« ( $§ 1$ Abs. 1 SGB II) und die Bedingungen für die Vereinbarkeit von Familie und Beruf ausgebaut werden. Insbesondere Alleinerziehende können durch die Kinderbetreuungssituation nicht ausreichend am Arbeitsmarkt teilhaben, daher sollen Betreuungsgelder und die erforderliche Infrastruktur ausgebaut werden (Hartz-Kommission 2002: 88). Selbst wenn für Eltern eines Kleinkindes Maßnahmen zur Wiedereingliederung und eine Berufstätigkeit als unzumutbar gelten, »kann es sinnvoll sein, im Rahmen Ihrer Möglichkeiten an der Beseitigung der Ausnahmesituation mitzuwirken und sich beispielsweise um einen Betreuungsplatz auch für Ihr unter drei Jahre altes Kind zu bemühen.«(BA 2013b: 22, BA 2008b: 19) Daneben steht das Gesetz zur Förderung der Ausbildung und Beschäftigung schwerbehinderter Menschen in einem inhaltlichen Zusammenhang mit der Hartz-Gesetzgebung (und der Agenda 2010): Es reagierte auf eine wachsende Zurückhaltung von Arbeitgebern bei der Ausbildung schwerbehinderter Jugendlicher (Weyand/Düwell 2005: 47). Behinderte Personen sollen nach dem Abs. 1 SGB II stärker in Arbeit einbezogen werden, um Benachteiligungen zu überwinden, denn eine geringe Qualifikation wird bei dieser Personengruppe als Hauptursache für die Arbeitslosigkeit angesehen (BA 2013e: 12). Die deutsche Aktivierungspolitik setzt hier zunehmend auf den ersten Arbeitsmarkt.

Weiter ist es ein Anliegen, gerade junge Menschen (unter 25 Jahren) in den Arbeitsmarkt zu integrieren. Je länger sie außerhalb des Arbeitsmarktes stehen, desto schlechter sind die Bedingungen für eine erfolgreiche Integration in die Gesellschaft im Sinne des Diskurses. So sei die steigende Jugendarbeitslosigkeit »besorgniserregend « (Hartz-Kommission 2002: 38) und man versuche »der Arbeitslosigkeit den Nachwuchs zu entziehen.« (BA 2006: 43) ${ }^{16}$ Unter 25-Jährige

16 Im Dezember 2013 hat Deutschland mit 7,7 Prozent die niedrigste harmonisierte Arbeitslosenquote für 15- bis 24-Jährige im EU-Durchschnitt (eurostat 2013). 
sollen ein aktivierendes Angebot erhalten, »das ihnen einen Einstieg ins Arbeitsleben und somit gesellschaftliche Teilhabe ermöglicht«, denn: »JobCenter übernehmen die Verpflichtung dafür zu sorgen, dass kein Jugendlicher ohne eine aktive beiderseitige Suche nach einer Praktikums- oder Ausbildungsstelle zu Hause sitzt und Transferleistungen erhält. « ${ }^{17}$ (Hartz-Kommission 2002: 25) Eine umfassendere Betreuung der Jugendlichen soll unter anderem ein höherer Betreuungsschlüssel (je eine Fachkraft für 25 Jugendliche) ermöglichen, der es erlaube »sich um jeden Jugendlichen intensiv zu kümmern, so dass uns keiner mehr durchs Netz geht.« (BA 2006: 52) Auch am anderen Ende des Altersspektrums der Erwerbsfähigen gilt es verstärkt, die Menschen in Arbeit zu bringen: »Ältere bewerten ihre Arbeitserfahrungen positiv, fühlen sich aber weniger gebraucht und sind auch isolierter « (BA 2008a: 46) - so bedürfen gerade sie einer Hilfestellung zur Arbeitsaufnahme. Grundsätzlich sei es eine wichtige Aufgabe für Arbeitsmarkt- und Beschäftigungspolitik in Deutschland und Europa, den Verbleib von älteren Beschäftigten in der Erwerbsarbeit zu sichern respektive sie zu reintegrieren, da der demographische Wandel zunehmend spürbar werde und dazu führe, dass die Nachfrage das Arbeitskräfteangebot in immer mehr Branchen übersteige (Hartz-Kommission 2002: 26). Ältere Beschäftigte sollen möglichst lange arbeiten (Verlängerung der Lebensarbeitszeit). Die Rolle der jungen Alten, die sich wenn nicht in Erwerbstätigkeit, dann im Ehrenamt verdingen, wird im Altersaktivierungsdiskurs proklamiert und dabei $>$ Alte $<$ ab 50 und meist unter 65 Jahren angesprochen (Dyk/Lessenich/Denninger/Richter 2010: 30). Aktivitäten, für die die adressierten Älteren ihre Potentiale nutzen sollten, werden aus der Perspektive des gesamtgesellschaftlichen Nutzens betrachtet; deshalb kommen ältere Personen, die weniger leistungsfähig sind, in diesem Diskurs kaum vor (Dyk et al. 2010). So werden im Rahmen des SGB II Personen mit sozialen und gesundheitlichen Beeinträchtigungen, Alleinerziehende oder teilerwerbsfähige Personen zur Integration in den ersten Arbeitsmarkt adressiert, die etwa in Dänemark in dauerhaften öffentlich-subventionierten und sozialpolitisch motivierten Beschäftigungsverhältnissen abgesichert werden (Konle-Seidl/Lang: 2006).

Zusammengefasst überträgt die ausgeweitete Definition von Erwerbsfähigkeit die Fähigkeit zur Selbstversorgung und damit eine Individualverantwortung auf einen quantitativ und qualitativ vergrößerten Personenkreis.

17 Die SGB II-Träger werden im Bericht der Kommission, »Moderne Dienstleistungen am Arbeitsmarkt« (Hartz-Kommission 2002) als »JobCenter« bezeichnet, in weiteren Texten und dem öffentlichen Diskurs jedoch als »Jobcenter«. 


\subsubsection{Disziplinierungszuwachs in Folge der Klassifizierung in eine Erwerbsfähigen-Nichterwerbsfähigen-Dichotomie}

Wenn in einer proklamierten Vollbeschäftigungsgesellschaft Individuen eigeninitiativ und eigenverantwortlich erwerbstätig oder erwerbslos sind - so die Prämisse des Aktivierungsdiskurses - entspricht Arbeitslosigkeit dieser Rationalität folgend abweichendem Verhalten. Denn prinzipiell wird sowohl den sich einfügenden als auch den abweichenden Individuen das Potential zur Integration in den Arbeitsmarkt zugesprochen. Menschen, die nicht einer Erwerbstätigkeit nachgehen, leben außerhalb der Norm und bilden ein Potential, um die Annahmen des Diskurses in Frage zu stellen beziehungsweise den Diskurs zu festigen, wenn Abweichung als missglückte Integration verstanden wird. Die Entdeckung von Devianz erzeugt weiter soziale Reaktionen, die dazu führen können, entsprechende Personen zu stigmatisieren, zu bestrafen und zu isolieren, auf die mittels (Re-)Integration reagiert wird. Die Weichenstellung zur Integration erfolgt über die Klassifizierung in erwerbsfähige Hilfebedürftige und nicht erwerbsfähige Hilfebedürftige. ${ }^{18}>$ Gute Arme oder zulässig erwerbslos sind diejenigen, die nicht erwerbsfähig sind, weil sie nicht dazu in der Lage sind, über Tätigkeiten im ersten Arbeitsmarkt Nennenswertes zu ihrem Lebensunterhalt beizutragen. »Nur wer seinen Lebensunterhalt nicht aus eigener Kraft bestreiten kann, kann von der Gemeinschaft Hilfsleistungen erwarten.« (BMWA (ehemaliges) 2005b: 24) Im Diskurs sind diese Personen jedoch von geringer Relevanz, wobei die Ausweitung des Erwerbsbegriffs zu einer deutlichen Verringerung dieser Gruppe von Nichterwerbsfähigen führte. ${ }^{19}$ Gesetzlich findet sich die Gruppe der Nichterwerbsfähigen im SGB XII. Über dessen Regelungen wird ihr Lebensunterhalt sichergestellt. Diejenigen, die hilfebedürftig und gleichzeitig erwerbsfähig sind unterliegen dagegen dem SGB II und erhalten sozialstaatliche Leistungen, solange sie nachweisen können (die Beweispflicht liegt also bei ihnen), dass sie bestrebt sind, die Hilfebedürftigkeit zu überwinden und einer

18 Eine solche Differenzierung wurde schon in den bismarckschen Versicherungen vorgenommen, um den arbeitsfähigen Armen ins Zentrum einer Arbeiterschutzpolitik zu stellen. Diese Kategorisierung ermöglichte in `selbstverschuldet` und sunverschuldet Hilfebedürftige zu unterscheiden und forcierte den »Verdacht des Müßiggangs und der Faulheit« bei arbeitsfähigen Individuen ohne Beschäftigung (Zimmermann 2006: 61f.).

19 Vergleiche Kapitel 4.2.2 »Arbeitslosigkeit `bekommt ein Gesicht««: Aktivieren eines erweiterten Personenkreises. 
Erwerbstätigkeit nachzugehen. Besonders so genannte Langzeitarbeitslose stehen im Fokus des Aktivierungsdiskurses. Nach zwölf Monaten ohne Anstellung, Erwerbsfähigkeit und Hilfebedürftigkeit vorausgesetzt, gehört man zu dieser Gruppe und je länger der erwerbslose Zustand anhält, desto schlimmer gilt es dem Aktivierungsdiskurs, denn »Die dauerhafte Abhängigkeit von staatlichen Leistungen ist keine Lebensperspektive.« (BMWA (ehemaliges) 2005a: 42). >Schlechte Arme`, die ein inaktives Leben führen und keine Vorleistung erfüllen, müssen sich auf Sanktionen einstellen (BMWA (ehemaliges) 2005a: 42f.) Zudem erzeugen Äußerungen wie »Es gibt kein Recht auf Faulheit« von dem früheren Bundeskanzler Gerhard Schröder ein politisches Klima, in dem eine Hängemattenmentalität zur Ursache der Arbeitslosigkeit umgedeutet wird (Opielka 2003: 103). Diese Unterscheidung der Sozialleistungsempfangenden erinnert an Charles Murrays undeserving poor. Murray grenzt diese soziale Gruppe, die aufgrund nicht-akzeptierten Verhaltens wie mangelnder Selbstdisziplin in eine selbstverschuldete Hilflosigkeit geraten sind, von den deserving poor ab, die unterstützt werden sollten, da sie nicht durch eigenes Verhalten verschuldet, sondern durch Krankheit, Unfall oder Alter hilfebedürftig wurden.

»When I use the term sunderclass $\$ I am indeed focusing on a certain type of poor person defined not by his condition, for example, long-term unemployment, but by his deplorable behaviour in response to that condition, for example, unwilling to take jobs that are available to him.« (Murray 1996: 83)

Die Unterscheidung erinnert zudem an Castels Darstellung der Geschichte der sozialen Frage in Frankreich, in der er die Armenversorgung im Mittelalter thematisiert. Die durch eine Handicapologie geprägten, nicht arbeitsfähigen Armen sind durch Sozialfürsorge versorgt, wenn auch häufig unzureichend. Selbst wenn ihre Versorgung Schwierigkeiten bereitet, stellt dies die Organisation der Gesellschaft nicht grundsätzlich in Frage. Diejenigen, die arbeitsfähig sind und dennoch nicht arbeiten (klassischer Vertreter des Entkoppelten ist der Vagabund), sind aus der Zone der Fürsorge verstoßen (Castel 2000: 27, 410). Neben den strukturellen Auswirkungen der Arbeitslosigkeit ist diese auch für das Individuum schädlich diskursiviert: Sie stigmatisiere den Menschen und je länger er in dieser Situation lebe, desto schwerer und kostenintensiver sei eine Rückkehr ins Arbeitsleben (Hartz-Kommission 2002: 46) ${ }^{20}$ Wie existentiell die Bedrohung verhandelt wird, deutet folgendes Zitat an: »Arbeitslosigkeit belastet nicht nur

20 Siehe auch (Presse- und Informationsamt der Bundesregierung 2003: 14). 
die unmittelbar Betroffenen. Die weitreichenden Folgen treffen alle, weil Entwicklungspotenziale für heutige und zukünftige Generationen vergeudet werden." (Hartz-Kommission 2002: 37) Die Stigmatisierung des Anormalen dient zugleich der Aufrechterhaltung der Selbstdisziplinierung der Norm beachtenden Mehrheit der Transferleistungsbeziehenden. So geriert auch die Thematik des Missbrauchs öffentlicher Gelder einen Generalverdacht gegen Arbeitslose. In »Vorrang für die Anständigen - Gegen Missbrauch, »Abzocke« und Selbstbedienung im Sozialstaat « (BMWA (ehemaliges) 2005b) macht das BMWA auf Grundlage von Einzelfällen eine pauschale Kultur der »Abzocke« aus. Geschildert werden Fälle, in denen beispielsweise Einkommen und Vermögen oder LebenspartnerInnen, mit denen eine Bedarfsgemeinschaft existiert, nicht angegeben wurden. Die so genannte »Mitnahmementalität« (Clement in: BMWA (ehemaliges) 2005b: 3) existiere jenseits sozialer Schichten. Abhilfe schaffe die Unterstützung der Bevölkerung, besonders aber die Kontrolle der SGB-II-Träger:

»Die ständige Verfügbarkeit als Voraussetzung für den Leistungsbezug [...] ist keine Schikane. [...] Davon profitieren nicht zuletzt auch die Ehrlichen, denn immer wieder werden Sozialbetrüger, die nicht für die Aufnahme einer Beschäftigung zur Verfügung stehen, erwischt.« (BMWA (ehemaliges) 2005b: 12)

Dieser Sicht auf die Leistungsbeziehenden, widersprechen jedoch Studien, die aufgezeigt haben, dass SGB-II-Leistungsbeziehende überwiegend die gesellschaftlich-zentrale Bedeutung von Erwerb akzeptieren und selbst keinen rein instrumentellen Bezug zur Erwerbstätigkeit haben (Ludwig-Mayerhofer/Behrend/Sondermann 2009: 107). Ferner ist ein Großteil der Leistungsbeziehenden auch ohne äußere Motivation oder Disziplinierung eigeninitiativ, um eine Anstellung zu erlangen (Dörre et al. 2013, Grimm et al. 2013, Bescherer/Röbenack/Schierhorn 2008, Baethge-Kinsky et al. 2007). Darüber hinaus kann die Aktivierungsstrategie der Arbeitsverwaltung mit einer Krise für Erwerbslose verbunden sein, die aus dem Verlust von sinnstiftender Tätigkeit und damit verbundener Identifikation wie auch dem Verlust materieller Sicherheit resultieren kann (Sondermann 2013). Kratz konstatiert ferner, dass die institutionelle Handlungsmaxime der Aktivierung sogar zu einer Objektivierung des Unterstützungsbedarfs der Leistungsbeziehenden führt und damit einen Fremdwerden der individuellen Erwerbsbiographie sowie den Verlust der Handlungskontrolle befördert (2013: 467ff.).

Insgesamt zeigt die Subjektpositionierung der SGB-II-Leistungsempfangenden im Aktivierungsdiskurs (Kapitel 4.2), dass die Beschreibung von KundInnen 
passgenau die Eigenverantwortlichkeit transportiert sowie Individualisierung befördert und zugleich vom Machtungleichgewicht in der Praxis der Beschäftigungsförderung abstrahiert. Ferner wird mit den Hartz-Gesetzen die Responsibilisierung der Verantwortung für den individuellen Status der Erwerbslosigkeit auf einen quantitativ und qualitativ größeren Personenkreis übertragen, indem die Kriterien der Beschäftigungsfähigkeit erweitert und die Gruppe von alleinerziehenden Frauen, Schwerbehinderten, unter 25-Jährigen und über 50-Jährigen verstärkt von der Beschäftigungsförderung aktiviert werden sollen. In toto werden individuelle Faktoren der Erwerbslosigkeit analysiert und bearbeitet. Darüber hinaus befördert die Dichotomie in erwerbsfähige und nicht-erwerbsfähige Hilfebedürftige in einer Rahmung der Individualisierung und Responsibilisierung von Erwerbslosigkeit einerseits die (Selbst-)Disziplinierung, doch andererseits genauso die Stigmatisierung der Mehrheit der Norm beachtenden erwerbsfähigen und häufig erwerbswilligen Sozialleistungsempfangenden.

\subsection{UNTERNEHMERISCHE ELEMENTE DER ARBEITSMARKTPOLITIK}

Arbeitslosigkeit kann nicht allein durch ein ungenügendes Passungsverhältnis von Angebot und Nachfrage wie im neoklassischen Modell erklärt werden, auf Defizite in der Güternachfrage nach dem keynesianischen Modell oder mismatch-Theorien, die auf Diskrepanzen basieren zwischen den Anforderungen freier Stellen und den Fähigkeiten der zur Verfügung stehenden Arbeitskräften: Es handelt sich vielmehr um ein Konglomerat aus vielfältigen komplexen ökonomischen, lebensweltlichen und arbeitsmarktlichen Einflüssen und Rückkoppelungseffekten (Ludwig-Mayerhofer 2005). Doch das die im Aktivierungsdiskurs gewählte Arbeitsmarktstrategie auch Auswirkungen auf die Ökonomie hat, dürfte unbestreitbar sein. Denn Grundannahme des Diskurses ist, dass eine Vollerwerbsgesellschaft und die Beendigung des Leistungsbezuges für jeden einzelnen >erwerbsfähigen Hilfebedürftigen` möglich und erstrebenswert sind. Sie setzt damit eine in der Weltwirtschaftskrise der 1970er Jahre einsetzende Tendenz fort, wonach Arbeitslosigkeit vehement bekämpft wird, um mit einem hohen Beschäftigungsstand die europäische und nationale Wettbewerbsfähigkeit auf dem Weltmarkt zu demonstrieren (Abelshauser 2009a). So wird Arbeitslosigkeit als mangelnde Anpassung an Sachzwänge der Globalisierung gedeutet.

»Damit ist nicht nur eine ernste soziale Frage beschrieben, sondern auch ein untrüglicher Indikator für ein fundamentales wirtschaftliches Problem identifiziert. Ganz offenbar ge- 
lingt es dem sozialen System der Produktion nicht mehr vollständig, seine potenziell wertvollste Ressource, das menschliche Vermögen produktiv einzusetzen.« (Abelshauser 2009b: 33)

Auch im Aktivierungsdiskurs bauen soziale Sicherungssysteme auf Erwerbsarbeit auf und damit auch das Wohl des Einzelnen wie der Gesellschaft: Da die soziale Sicherung zu einem Großteil beitragsfinanziert (damit lohnsummenorientiert) ist, werde sich die Erwerbstätigkeit der größtmöglichen Zahl positiv auf die System-Funktionsfähigkeit auswirken. Durch eine den Beitragszahlern gegenüber zu große Anzahl an Empfängn sei dessen Leistungsfähigkeit eingeschränkt (Hartz-Kommission 2002: 39). Die »akuten Finanzierungsnöte« (Presse- und Informationsamt der Bundesregierung 2003: 5) ${ }^{21}$ resultierten weiter aus einem Missverhältnis zwischen Rentenansprüchen und den diese Altersbezüge erwirtschaftenden Beschäftigten auf Grund des demographischen Wandels, des hohen Konkurrenzdrucks der Unternehmen (Globalisierung) und eines stagnierenden Wirtschaftswachstums. Zudem gebe es Komplikationen bei der Abstimmung von Arbeitsangebot und -nachfrage, dem Matching: Einerseits verlaufe der Informationsfluss zwischen offenen Stellen, Lehrstellen und den danach Suchenden nicht optimal. Andererseits müssten Arbeit- und Ausbildungssuchende flexibel sein in Bezug auf Arbeitsort und Art der Tätigkeit. Den Informationsfluss zwischen Wirtschaft und Mensch sollen die SGB-II-Träger verbessern und zugleich mehr Flexibilität der Menschen einfordern.

Nun wird analysiert, welche arbeitsmarktpolitischen Strategien proklamiert werden, um die Erwerbsintegration zu forcieren (Kapitel 4.3.1) und welche Förderungen und Anforderungen hinsichtlich der Beschäftigungs- und Bildungsoptionen für erwerbslose Leistungsbeziehende (Kapitel 4.3.2) bestehen.

\subsubsection{Strategien zur Reduzierung der Erwerbslosigkeit}

»Unser Sozialsystem droht aus den Fugen zu geraten, weil es nicht mehr finanzierbar ist. Arbeit ist zu teuer geworden, weil die Lohnnebenkosten zu hoch sind.« (Presse- und Informationsamt der Bundesregierung 2003: 8) In Reaktion auf diese Lagebeurteilung steht die Schaffung »neuer Arbeitsplätze« und »zusätzlicher Beschäftigungsmöglichkeiten« im Vordergrund - was insbesondere über den Ausbau des Niedriglohnbereichs erfolgen soll, der primär einfache

21 So Gerhard Schröder im Vorwort; siehe auch im Folgenden (Presse- und Informationsamt der Bundesregierung 2003: 5f.). 
Dienstleistung bietet, und ein neues Verständnis von »lebenslangem Lernen« voraussetze (Hartz-Kommission 2002: 38f.). Es wurden Beschäftigungsformen installiert wie Zeitarbeit, Mini- und Midijobs, die die Lebenshaltungskosten nicht decken können. Falls die Voll- oder Teilzeitbeschäftigung weniger als das Existenzminimum erbringt, greifen ergänzende Sozialleistungen. Die Zahl dieser erwerbstätigen Leistungsbeziehenden ist von 23 Prozent in 2007 auf 29 Prozent in 2011 gestiegen, während die Anzahl der erwerbsfähigen Hilfebedürftigen in den letzten Jahren signifikant abgenommen hat (Bruckmeier/Eggs/Himsel/Trappmann/Walwei 2013: 2). Ein geringerer Lohn, verglichen mit vorhergehenden Tätigkeiten, ist laut Aktivierungspolitik kein Kriterium um die Zumutbarkeit einer neuen Arbeitsstelle abzuerkennen. Für den Bereich des Arbeitslosengeldes ist jedoch eine Einkommensstand-Sicherung vorgesehen, die mit voranschreitender Dauer der Arbeitslosigkeit sinkt. ${ }^{22}$ Innerhalb des Diskurses ist es von Bedeutung, Menschen im Beschäftigungssystem zu halten. Der verfügbare Bestand an Arbeitskräften soll es den Unternehmen ermöglichen, Labor Hording zu vermeiden und die Beschäftigtenzahl zu variieren. Auf diese Weise folge die Beschäftigtenzahl stärker und rascher der Konjunktur. Unter anderem ist Zeitarbeit ein wichtiger Bestandteil zur Erlangung der Vollbeschäftigung, denn ihr wird der sogenannte Klebeeffekt zugeschrieben (Hartz-Kommission 2002: 29), also ein Verbleib im Entleihbetrieb, in einem regulären Beschäftigungsverhältnis. Zwar hat Zeitarbeit durchaus zu einem numerischen Beschäftigungsaufbau beigetragen, doch erfolgt der Beschäftigungsgewinn auch zu Lasten von Arbeitsplätzen aus anderen Branchen (Jahn/Weber 2013). Darüber hinaus sind Klebeeffekte unter spezifischen Umständen möglich, jedoch ist die Integration in unbefristete Arbeitsverhältnisse für Arbeitnehmende wahrscheinlicher, die aus befristeten Anstellungen kommen (Achatz et al. 2013). ${ }^{23}$ Öffentlich geförderte Beschäftigung

22 Gemäß $\S 140$ Abs. 3 SGB III ist im ersten Quartal der Arbeitslosigkeit eine Minderung um mehr als zwanzig Prozent des dem Arbeitslosengeldanspruch zugrundeliegenden Einkommens zumutbar. Im zweiten Quartal eine Minderung um mehr als dreißig Prozent und sobald die Arbeitslosigkeit mehr als sechs Monate andauert, muss mindestens die Höhe des Arbeitslosendgeldes erreicht werden (60 Prozent bei Alleinstehenden, 67 Prozent bei Arbeitslosen mit familiären Verpflichtungen, jeweils des letzten Gehaltes).

23 Darüber hinaus gehen mit der Zeitarbeit erhöhte Prekaritätsrisiken einher und finden im Kontext von weitestgehend prekären Interessenvertretungs- und innerbetrieblichdemokratischen Teilhabeoptionen für Zeitarbeitsbeschäftigte statt (Artus 2014). In diesem Zusammenhang ist auch der tendenziell niedrigere gewerkschaftliche Organi- 
spielt im Diskurs eine marginale Rolle und soll nur in Übergangsphasen in strukturschwachen Regionen genutzt werden. Stringent wurde die öffentlich geförderte Beschäftigung (ohne Bürgerarbeit) um etwa ein Drittel auf 24,1 Prozent der Maßnahmenteilnehmenden reduziert (BA 2013e: 24).

Doch nicht nur dem Individuum auch der Wirtschaft wird eine Mitverantwortung am Transferleistungsbezug des Einzelnen zugesprochen, die ebenfalls von subjektivierenden Tendenzen getragen wird. Der gesamtgesellschaftlichen "Herausforderung« soll begegnet werden, indem sich eine »Allianz der Profis« bildet, die sich an der Beendigung oder Verhinderung von Erwerbslosigkeit mit einer »verbindlichen Leistung « beteiligt: »)Profi< sind für uns alle, die durch ihre Kompetenz in unserem Gemeinwesen dazu beitragen können, dass aus sinnvollen und zum Teil unerledigten Aufgaben für die Gesellschaft Beschäftigung für Arbeitslose entsteht.« (Hartz-Kommission 2002: 286f.) So sollen Unternehmen Verantwortung übernehmen, indem sie Entlassungen durch »betriebliche Atmungsinstrumente « (Hartz-Kommission 2002: 28) vermeiden. Weiter soll die politische, wirtschaftliche und mediale Öffentlichkeit helfen das »Verantwortungsgefühl« (Hartz-Kommission 2002: 87f.) zu stärken.

»Wenn wir den Kampf gegen die Arbeitslosigkeit gewinnen wollen, dürfen wir uns nicht passiv verhalten. Wir können nicht weiterhin den Status des Beobachters einnehmen. Arbeitslosigkeit in ihren weitreichenden Folgen betrifft auch uns." (Hartz-Kommission 2002: 286)

Den SGB-II-Trägern stehen zur Prävention von Arbeitslosigkeit »Übergangsarbeitsmärkte« zur Verfügung. So gewinnt der Übergangssektor zunehmend an Bedeutung, der als Vorstufe des dualen oder staatlich-schulischen Ausbildungssystems fungiert: hier werden vornehmlich Jugendliche ohne Schulabschluss oder mit Hauptschulabschluss in maßnahmenförmig organisierten unterschiedlichen Bildungsangeboten verschiedener Träger qualifiziert (Trinczek 2013: 211). Diese Vorstufe kann auch in Form der hier untersuchten Maßnahmen Sozialer Aktivierung stattfinden. Die Übergangsarbeitsmärkte beinhalten so zum Beispiel Nachqualifizierung, aktive Sozialplanmaßnahmen, Förderung der Selbstständig-

sationsgrad von atypisch Beschäftigten zu sehen (Artus 2013: 109). Relevant für eine gewerkschaftliche Interessenvertretung im Niedriglohnsektor kann sich besonders eine Anpassung der kulturellen Orientierungen von Gewerkschaften an die Beschäftigten in vielfältigen Arbeitsumgebungen erweisen (Artus 2011). 
keit, Arbeitsplatzanpassungen für Ältere oder leistungsgeminderte Beschäftigte (Hartz-Kommission 2002: 46).

So soll Erwerbslosigkeit unter anderem durch kurze Verweildauer zwischen Kündigung und nächster Tätigkeit eingedämmt werden (§ 1 Abs. 1 SGB II). Der SGB-II-Träger hat die vorrangige Aufgabe, individuelle Integrationshemmnisse zu bewältigen und in »Übergangsphasen« eine (möglichst kurze) Betreuung der Arbeitslosen zu leisten (Hartz-Kommission 2002: 97), durch eine schnelle Vermittlung in Erwerbstätigkeit über eine an individuellen Interessen orientierte und marktabhängige Stellenrecherche. In der möglichst kurzfristigen Vermittlung zeigt sich die Annahme, dass jeder erwerbsfähige Mensch in den ersten Arbeitsmarkt integrierbar sei, wenn das Qualifikationsverhältnis ausgeglichen ist. Ferner soll Arbeitslosigkeit verhindert werden durch leichtere Übergänge zwischen Arbeits-, Bildungs- und Erziehungszeiten sowie die Aufhebung von Beschränkungen im Arbeitsmarktzugang für strukturell benachteiligte Personen. Summa summarum wird jedoch mit >regulären Z Zeitabschnitten ohne Anstellung im Verlauf einer Erwerbsbiographie in Zukunft gerechnet. Deshalb ist angedacht, die Arbeitslosen- in eine »Beschäftigungsversicherung « zu reformieren, so dass Zeiten ohne Gelderwerb und Einkommensrisiken zwischen Beschäftigungsverhältnissen abgefedert werden können (Hartz-Kommission 2002: 47).

Doch dem Arbeitsmarkt soll auch von anderer Seite Potential zugeführt werden: Indem Arbeit außerhalb von Arbeitsverträgen, die sogenannte »Schwarzarbeit«, möglichst stark eingedämmt wird. Über die steuerliche Abzugsfähigkeit der Kosten privater Dienstleistungen und strikte Sanktionen soll ein weiteres Beschäftigungspotential erschlossen werden. Mittel dazu sind »Ich- und FamilienAG « sowie der »Mini-Job«, die gerade einfache Arbeiten attraktiver gestalten sollen (Hartz-Kommission 2002). »Es gibt auch ein Recht auf einfache Arbeit. Viele Menschen wollen oder können nicht weiter qualifiziert werden. (HartzKommission 2002: 41) Letztlich soll die Motivation zur Flexibilität bei Arbeitsplatz-, Berufs- und Ortswechsel honoriert werden.

Mithin ist in den vergangenen Jahren die Bedeutung atypischer Beschäftigungsformen wie Teilzeitbeschäftigung, geringfügige nicht sozialversicherungspflichtige Beschäftigungen (Minijobs), befristete Beschäftigungen sowie Leiharbeit angestiegen (Ludwig-Mayerhofer 2012, Grimm et al. 2013, Hohendanner/Walwei 2013). ${ }^{24}$ Zwar sind in Deutschland im vergangenen Jahrzehnt bei

24 Interessant sind in diesem Kontext auch die prekären Lebens- und Arbeitsbedingungen von (temporär) transnational arbeitenden Posted Workern (Staples/Trinczek/Whittall 2013). 
Frauen und Älteren ein Rückgang der Nichterwerbstätigkeit bei gleichzeitigem Anstieg von atypischen Beschäftigungsverhältnissen zu konstatieren, was durchaus die Integrationsstrategie des Aktivierungsdiskurses stützt, dagegen stehen insbesondere jüngere und niedrigqualifizierte Arbeitnehmende in marginaler Teilzeitbeschäftigung und befristeter Beschäftigung, während der Anteil der Normalarbeitsverhältnisse hier stark rückläufig war (Allmendinger/Giesecke/ Hipp 2012).

Zusammengefasst werden für die Reduzierung von Erwerbslosigkeit die Strategien verfolgt, Anstellungsmöglichkeiten für einfache und geringfügige Tätigkeiten als flexible Instrumente zur Verfügung zu stellen, Übergangsarbeitsmärkte zu schaffen und durch schnelle Übergänge Erwerbslosigkeit zu verhindern. Öffentlich geförderte Beschäftigung als dauerhafte Einrichtung für spezifische Personengruppen wird nicht anvisiert. Es zeigt sich, dass prekäre Beschäftigungsformen sowie der Anteil an erwerbstätigen SGB-II-Leistungs-Beziehenden zunehmen, während die Arbeitslosenquote insgesamt abnimmt.

\subsubsection{Zumutbare Beschäftigungsvielfalt versus Bildung}

Die Ausweitung atypischer Beschäftigungen geht mit der Zielsetzung einher, die möglichen Berufsfelder von Erwerbslosen zu expandieren. Diese sollen, wenn die Arbeitsplatzsuche in der ursprünglichen Domäne aussichtsarm ist, nicht nur in ausbildungsadäquaten Beschäftigungsverhältnissen tätig werden. Stellen sollen dann verstärkt in Bereichen offeriert werden, die den Qualifikationen und Fähigkeiten der SGB-II-Leistungsbeziehenden verwandt sind, sogenannten JobFamilien. Migrations- und Besetzungspfade sollen infolgedessen geöffnet (Hartz-Kommission 2002: 72), die Flexibilität der beruflichen Tätigkeit gefördert werden. Denn auch bei momentan guter Beschäftigungslage in einer Sparte, wird die Möglichkeit der kurzfristigen Umorientierung von ArbeitnehmerInnen bereits antizipiert sowie die Individuen an mögliche Tätigkeitswechsel gewöhnt. Nach $\S 10$ SGB II ist dem Leistungsbeziehenden jede Arbeit zuzumuten, auch wenn sie nicht der Ausbildung entspricht, sie im Vergleich zur vorherigen Erwerbsarbeit als »geringer wertig« anzusehen ist oder deren »Arbeitsbedingungen ungünstiger« sind.

Die "persönlichen Lebenschancen" (Presse- und Informationsamt der Bundesregierung 2003: 20) Erwerbsloser und deren Zugang zur Erwerbsarbeit sollen über Bildung sichergestellt werden. Daher wird sozialer Ungleichheit in der Bildungsbeteiligung entgegenwirkt und ein möglichst hohes Bildungsniveau sowie national verbindliche Bildungsstandards gefördert, die unteren Gesellschafts- 
schichten zu Gute kommen (Presse- und Informationsamt der Bundesregierung 2003: 26f.). Dem liegt die Vorstellung zu Grunde, dass die Gesellschaft eine Wissensgesellschaft ist, in der Wissen die klassisch-ökonomischen Eigenschaften eines handelbaren Gutes besitzt und als Qualifikation zur Verrichtung von Erwerbstätigkeit unerlässlich ist. Somit beinhaltet »aktivierende Hilfe« die Mobilisierung eines vorausgesetzten Handlungspotentials, um Menschen in Arbeit zu integrieren. »Deutschlands Reichtum ist das Wissen und Können seiner Menschen. Nur ein hoher Wissens- und Ausbildungsstand garantiert, dass Deutschland technologisch in der Welt einen Spitzenplatz behauptet.« (Presse- und Informationsamt der Bundesregierung 2003: 26)

Die Bedeutung der Bildung steht im Aktivierungsdiskurs speziell bei erwerbslosen Jugendlichen im Vordergrund. Gemäß der Diskurslogik waren die Jugendlichen nicht oder zu kurz in die Gesellschaft integriert und je länger sie außerhalb des Erwerbslebens stehen, desto schwieriger sei es für sie, ein >normales Leben` zu führen. Ihre Beschäftigungsfähigkeit liege als Potential brach. Die persönlichen »Leistungs- und Entwicklungsmöglichkeiten« unter 25-Jähriger, insbesondere jener, die »weniger gute materielle, soziale oder kulturelle Voraussetzungen mitbringen«, sollen in Zusammenarbeit mit den SGB-II-Trägern ausgebaut werden (Hartz-Kommission 2002: 106). Ausbildungsberufe sollen von den SGB-II-Trägern in Abhängigkeit von der Marktrelevanz geschaffen werden und das Schulsystem eine höhere »Praxisorientierung " (Hartz-Kommission 2002: 106f.) erhalten. Unausgeführt bleibt, was unter individuellen Entwicklungsmöglichkeiten verstanden wird. Die Soziale Sicherung hat demgegenüber nicht den Anspruch oder ist nicht dazu in der Lage, »persönliche Lebenschancen« des Leistungsempfängers zu realisieren. Zugleich wird die Verantwortung für das Wohl des Einzelnen im Diskurs beim Individuum verortet.

Eine Verknüpfung von individueller Bildung und Volkswirtschaft charakterisiert den Aktivierungsdiskurs: »Bildung ist der Schlüssel zu individuellem beruflichen Erfolg, der auch der Volkswirtschaft zugutekommt. [...] Nur so können bestehende Arbeitsplätze gesichert und neue geschaffen werden.« (Presse- und Informationsamt der Bundesregierung 2003: 28) Wie eingangs erwähnt wird die berufliche Qualifikation - anders als noch bei ABM für ArbeitslosenhilfeEmpfänger - nicht berücksichtigt.

»Eine der Ausbildung oder früheren Tätigkeit [...] nicht entsprechende Tätigkeit ist spätestens dann zumutbar, sobald festgestellt werden kann, dass eine berufliche Statusminderung unvermeidlich ist, um den Bezug von Arbeitslosengeld zu beenden.« (Hartz-Kommission 2002: 95) 
Hier wird ersichtlich, dass das Bildungs- hinter dem Erwerbstätigkeitsmotiv rangiert. Wenngleich im Aktivierungsdiskurs eine Achse der Integration in den Arbeitsmarkt über Bildung verläuft, kann dem Bildungsparadigma zuwidergehandelt werden, wenn Menschen in ungelernte Tätigkeiten wechseln. Bei Ablehnung einer Stelle muss der Leistungsbeziehende zudem beweisen, dass die abgelehnte Beschäftigung unzumutbar war; damit wird die Beweispflicht umgekehrt (Hartz-Kommission 2002: 24). ${ }^{25}$

Insgesamt ist die Arbeitsmarktpolitik (Kapitel 4.3) dadurch gekennzeichnet, dass die zur Reduzierung von Erwerbslosigkeit verfolgten Strategien wie flexible Beschäftigungsinstrumente im Niedriglohnbereich zwar begleitet werden von einer abnehmenden Arbeitslosenquote, jedoch zugleich der Anteil prekärer Beschäftigung sowie erwerbstätiger SGB-II-Leistungsbeziehender zugenommen haben. Ferner wird Bildung als Schlüssel zu individuellem Erfolg in einer Wissensgesellschaft diskursiviert. Zugleich kann diese Schlüsselrolle der Bildung allerdings geschmälert werden, da sie im Diskurs als an die Marktnachfrage gekoppelt erscheint und somit eine Zumutbarkeit von ungelernten Tätigkeiten gesehen wird, wenn in der gelernten Tätigkeit für das Individuum keine rasche Erwerbsintegration möglich ist.

\section{4 "ALLES IST SOZIAL, WAS ARBEIT SCHAFFT «: ${ }^{26}$ SOZIALE TEILHABE ÜBER ERWERB}

Im Folgenden wird entwickelt wie sich Erwerbsarbeit im Sinne des Diskurses ausgestalten soll. Im Aktivierungsdiskurs ist die Frage nach der Art der Tätigkeit eindeutig der Aufnahme einer Erwerbsarbeit nachgeordnet. Über diese vermittelt sich soziale Teilhabe an mehr oder weniger zentralen Bereichen und Ressourcen der Gesellschaft. Der arbeitslose Kunde wird zum Unternehmer seiner Beschäftigungsfähigkeit erklärt. Das Motiv der >an sich guten Arbeitく zieht sich durch

25 In toto kommt den Sanktionen aufgrund der Weigerung der Aufnahme oder Fortführung von Arbeit, Ausbildung oder Maßnahme jedoch 2007-2012 ein wesentlich geringerer Anteil (2012 mit 13,4 Prozent) als durch Meldeversäumnissen bei den SGB IITrägern zu (2012 67,9 Prozent) (Apel/Engels 2013: 10).

26 »Sozial ist, was Arbeit schafft« stammt von Hans Tietmeyer und mit demselben Slogan zog Edmund Stoiber 2002 in den Bundestagswahlkampf. Der damalige Wirtschaftsminister Wolfgang Clement übernahm die Aussage 2002. 
den dargestellten Diskurs. Zielsetzung im Vierten Gesetz für moderne Dienstleistungen am Arbeitsmarkt (Deutscher Bundestag 2003b) ist die Aufnahme jeglicher Tätigkeit. Zuvor wurde in $§ 1$ Abs. 2 Job-AQTIV-Gesetz, das sich auf damalige Arbeitslosenhilfe-Empfänger bezog, noch der Anspruch erhoben, »unterwertiger Beschäftigung entgegenzuwirken« (Deutscher Bundestag 2001: 3444). In Folge der Hartz-Gesetzgebung soll die Aktivität der Arbeitslosen in erster Linie der Hinwirkung auf die Erwerbsarbeitsaufnahme beziehungsweise gemeinnützige Arbeit in Form von Fördermaßnahmen dienen. Ehrenamtliches Engagement war mit dem Job-AQTIV-Gesetz nur zu vereinbaren, wenn es die »berufliche Eingliederung nicht beeinträchtigt« (Job-AQTIV-Gesetz, Änderung des $\S 118$ a SGB III). Somit ist nicht die (ehrenamtliche) gemeinnützige Tätigkeit von Bedeutung für die Integration, sondern ausschließlich eine Tätigkeit im ersten Arbeitsmarkt. Eine Studie, die sich mit der Sichtweise der Leistungsempfänger befasst, konstatiert, dass Tätigkeiten neben der Erwerbstätigkeit, die aus der Sicht des Einzelnen identitätsstabilisierend erscheinen, nach dem SGB II gesellschaftlich unerwünscht und sanktionswürdig sind. ${ }^{27}$ Zugleich wird die Erwerbsfunktion, ausreichende Subsistenzmittel bereitzustellen, aufgeweicht. Es wird akzeptiert, dass trotz Arbeit ein SGB-II-Leistungsbezug bestehen kann (»Aufstocken«). Es sei besser, für wenig Geld zu arbeiten, als ausschließlich hilfebedürftig zu sein. Die viel diskutierte Prekarisierung von Arbeit, ${ }^{28}$ der zufolge Arbeitsverhältnisse trotz hohem persönlichem Einsatz nicht den Lebensunterhalt decken oder keine weiterführende Perspektiven bieten, wird im Aktivierungsdiskurs eher forciert. Die von dem angelsächsischen welfare to work adaptierte Arbeit für Sozialhilfe akzeptiert auch die Entstehung einer Schicht von working poor. Die Verbreitung von Einkommensarmut könnte zu einer wachsenden sozialen Ungleichheit führen. Während 199213 Prozent der Erwerbstätigen atypisch beschäftigt waren, steigt ihr Anteil 2012 auf 22 Prozent (Statistisches Bundesamt 2013: 339f.). Die Aktivierungspolitik zielt darauf Arbeitskraft zu rekommodifizieren und führt »zu einer dauerhaften Ausgrenzung immer größerer Bevölkerungsgruppen« (Wolf 2006: 1090). Denn zum einen beeinträchtigen geringere

27 Siehe hierzu den Typ des konventionellen Nicht-Arbeiters, den Bescherer, Röbenack und Schierhorn untersuchen (Bescherer et al. 2008).

28 Wegbereiter und Kritiker des Konzeptes der Prekarisierung, der Verwundbarkeit und der Exklusion beziehungsweise »désaffiliation« war der französische Soziologe Robert Castel insbesondere mit seinem 1995 erstmals erschienen Werk »Die Metamorphose der sozialen Frage« (2000) (Castel/Dörre 2009, Castel 2011). Ein weiterer wichtiger Vertreter der regen Diskussion war Pierre Bourdieu (1998, 2000). 
erwirtschaftete finanzielle Ressourcen die Teilhabe am kulturellen und gesellschaftlichen Leben und zum anderen führen (Selbst-)Stigmatisierungs- und (Selbst-)Ausgrenzungsprozesse der Erwerbsfähigen-/Nichterwerbsfähigen-Dichotomie auch zu Beschränkungen sozialer Teilhabe. Zwar war eine gesteigerte Erwerbsbeteiligung im Diskurs intendiert und stellte sich auch ein, doch geht diese mit einer wachsenden Lohnungleichheit und vermehrten atypischen Beschäftigung einher (Klinger et al. 2013) ${ }^{29}$ In Kenntnis dieser Zusammenhänge haben CDU, CSU und SPD in ihren Koalitionsvereinbarungen auch Beschlüsse zu einem flächendeckenden gesetzlichen Mindestlohn getroffen, der nach dem Gesetzentwurf zumindest für Erwerbstätige greift. ${ }^{30}$ Im Koalitionsvertrag wird weiter Bezug genommen auf einen Diskurs um Gute Arbeit: „Gute Arbeit muss sich einerseits lohnen und existenzsichernd sein." (CDU Deutschlands/CSULandesleitung/SPD 2013: 48). Ob in den Aktivierungsdiskurs auch diese Aspekte eingehen und weitergeführt werden, indem Anforderungen an die Erwerbsverhältnisse gestellt werden wie eine gesteigerte Arbeitsqualität, Ermöglichung von individueller Zukunftsplanung weitgehend unabhängig von konjunkturellen Schwankungen, Chancen zur Vermögensbildung, weiteren Möglichkeiten des wirtschaftlichen und sozialen Aufstiegs und Mitbestimmung oder gar eine Koppelung von Erwerbsarbeit und würdevollem Leben ${ }^{31}$ bleibt abzuwarten.

29 Dies beginnt bereits mit Entwicklungen der Destandardisierung von Beschäftigungsverhältnissen sowie der Prekarisierung und Flexibilisierung von Arbeit über die Rainer Trinczek einen Überblick bietet (2011).

30 Seit 2015 gilt ein flächendeckender gesetzlicher Mindestlohn von 8,50 Euro pro Stunde in Deutschland. Zunächst mit Ausnahmeoptionen der tariflichen Abweichung, die ab 2017 entfallen (CDU Deutschlands/CSU-Landesleitung/SPD 2013: 48f.). Dieser soll nach dem aktuellen Gesetzentwurf jedoch zeitweise nicht bei der Einstellung von Langzeitarbeitslosen greifen (Dettmer/Gathmann/Wittrock 2014).

31 Eine die Menschenwürde berücksichtigende Lohnhöhe sehen etwa die UN-Menschenrechtskonvention von 1966 und der Art.23 III der Allgemeinen Erklärung der Menschenrechte von 1948 vor. Kantel führt an, dass es im Zuge der Gesetzesreform zur Absage an ein menschenwürdiges Leben kommt: Im alten BSHG wie im neuen SGB XII wird ein menschenwürdiges Leben »ermöglicht«, nicht garantiert. Darüber hinaus sieht das neue Gesetz nur mehr eine `Entsprechung`menschenwürdigen Lebens vor, denn das Ziel könne nur über ein Leben unabhängig von sozialstaatlichen Leistungen erreicht werden (Kantel 2004). 
Zusammengefasst nimmt im Aktivierungsdiskurs die Erwerbsintegration die zentrale Stellung ein, auch wenn die Erwerbstätigkeit die Funktion der Bereitstellung von Subsistenzmitteln nicht vollständig erreicht. Weiter müssen die zumutbaren Tätigkeiten für die Unternehmerin respektive den Unternehmer der eigenen Beschäftigungsfähigkeit nicht ausbildungsadäquat sein. Insgesamt weist die aktivierende Arbeitsmarktpolitik so eine Rekommodifizierung der Arbeitskraft auf, der eine dauerhafte Ausgrenzung von Personen zugeschrieben werden kann. Die Minderung finanzieller Ressourcen sowie (Selbst-)Stigmatisierungserfahrungen von Erwerbslosen können die kulturelle und gesellschaftliche Teilhabe beeinträchtigen.

\subsection{Hybride Momente des AKtivierungsdiskurses}

Wie in der bisherigen Darstellung des Diskurses ausgeführt, existieren diskursimmanente Widersprüche, die sich mit der Vereinnahmung widerstreitender Positionen durch den Diskurs erklären lassen und im Folgenden als Hybridisierungstendenzen bezeichnet werden. Wie im politischen Machtdiskurs, den Srubar beschreibt, handelt es sich um eine Sinnselektion zum Ausschluss alternativer Sinnbildung, doch nicht vornehmlich in Form der Exklusion abweichender Deutungsmuster, sondern

»in der Immunisierung der sinngenerativen Regeln gegen eine sozusagen feindliche Übernahme durch ein anderes sinngeneratives Regelwerk oder [...] gegen die Deterritorialisierung des eigenen Rhizoms (Deleuze/Guattari 1992), beziehungsweise gegen eine Übersetzung des eigenen Diskurssystems in die vergewaltigenden Termini eines anderen (Lyotard 1994).«(Srubar 2006: 9)

Zur Selbstreproduktion und -transformation des Machtdiskurses werden Mittel der Semantikbildung, der medialen Wirklichkeitskonstruktion und struktureller Kommunikationskonditionierung aktiviert. Die Verklammerung der Gegensätze führt zu einer Immunität gegenüber Möglichkeiten, Protest- und Weigerung zu artikulieren, »weil sie keine >rechten Worte〈 dafür mehr bietet.« (Srubar 2006: 10) Diese Betrachtung ist insofern relevant, als die Frage gestellt werden kann, ob die Hybridisierungstendenzen ebenfalls bei den diskurstragenden Institutionen im Sinne des Diskurses auftreten oder ob der Aktivierungsdiskurs nicht nur an seinen Enden >ausfranst<, sondern negiert wird. Die Hybridisierungstendenzen im politisch-rechtlichen Diskurs sollen an zwei Beispielen verfolgt werden. Im ersten Fall wird die traditionelle Form der Integration in Gesellschaft über 
Familie (Kapitel 4.5.1), inklusive ihrer Aufhebung, in den Diskurs einbezogen. Im zweiten Fall wird das Inklusionsprinzip der `klassischen Arbeit` (Kapitel 4.5.2), die über den Erwerb monetärer Mittel in die Gesellschaft führen soll, überformt durch eine Beschäftigungsform, die diese Ressourcen nicht erbringt. Stattdessen ist letzterer mit dem exkludierenden Stigma der selbstverschuldeten Hilfebedürftigkeit behaftet.

\subsubsection{Bedarfsgemeinschaft und Familie: Menschen sollen füreinander einstehen}

Gemäß dem SGB II gibt es eine Verpflichtung der Menschen, die miteinander leben, für den gegenseitigen Lebensunterhalt einzustehen. Während in der Arbeitslosenhilfe nur die Ehepartnerin und der Ehepartner als versorgungspflichtig galten, sind dies seit 2005 alle Personen, ${ }^{32}$ die in einem gemeinsamen Haushalt leben. So sollen sie »in eigener Verantwortung alle Möglichkeiten nutzen, ihren Lebensunterhalt aus eigenen Mitteln und Kräften zu bestreiten. Erwerbsfähige Hilfebedürftige müssen ihre Arbeitskraft zur Beschaffung des Lebensunterhalts für sich und die mit ihnen in einer Bedarfsgemeinschaft lebenden Personen einsetzen.« ( $\$ 2$ Abs. 2 SGB II) Diese Verpflichtung greift, wenn »[e]in wechselseitiger Wille, Verantwortung füreinander zu tragen und füreinander einzustehen « (\$7 Abs. 3a SGB II) besteht. Der wechselseitige Wille wird angenommen, sobald Partner länger als ein Jahr oder mit einem gemeinsamen Kind zusammenleben, Kinder oder Angehörige im Haushalt versorgen oder über Einkommen oder Vermögen des anderen verfügen können. ${ }^{33}$ Daneben sieht der Diskurs die gegenseitige Verantwortung und Stärkung familiärer Beziehungen als vereinbar mit dem Postulat der Flexibilität und Mobilität. Familiäre Bindungen, die nicht ausschließlich auf einer finanziellen Verantwortung gründen, benötigen Zeit für Gemeinsames und eine gewisse räumliche Nähe. Diese Konstellation lässt sich theoretisch mit den Überlegungen Berger und Luckmanns einholen und als Nihilierung einer konträren Wirklichkeitskonstruktion beschreiben (Berger/Luckmann 2004: 170f.), indem Widersprüche zur bestehenden Sinnwelt sich zu Axiomen derselben formen. Problematisch könnte sich diese Erwartung an ein »tra-

32 Also nicht-eheliche Partnerschaften, Kinder und Großeltern, die zusammenleben.

33 Die Praxis, zusammenlebende Paare zu einer eheähnlichen Einstandsgemeinschaft zu erklären, widerspricht höchstrichterlichem Recht, denn nach dem BGB besteht kein unterhaltsrechtlicher Anspruch bei unverheirateten Paaren (Wolf 2006: 1091). 
diertes Familienbild $\aleph^{34}$ durch die Lebenspraxis der vergangenen Dekaden gestalten: Während in der Feudalgesellschaft die Reproduktionsfunktion hauptsächlich in der Großfamilie mit mehreren Generationen erfolgte, fand in Zeiten der Industrialisierung eine Differenzierung der Produktions- und Reproduktionstätigkeiten statt. Die Kernfamilie, bestehend aus den Eltern und den Kindern, wird zur dominierenden Familienform. »Die Kernfamilie wurde von den anderen Einheiten der Verwandtschaft funktional isoliert, wodurch die Kernfamilien stärker von dem Erwerbeinkommen ihrer Mitglieder abhängig wurden.« (Hill/ Kopp 2013: 258) In der weiteren Pluralisierung der Familienformen, Lebensformen und sozialen Werte nimmt die Bedeutung der Familie als Wirtschaftsgemeinschaft weiterhin ab (Beck-Gernsheim 1986: 212 in Hill/Kopp 2013: 263). Über die Normarbeitsplätze mit gesetzlicher Absicherung und relativ gut bezahlter Arbeit für einen Großteil der Bevölkerung lösten sich die Personen immer mehr aus den traditionellen Familienkonstellationen (Individualisierung). Da grundlegende Sicherheitsstandards durch den Sozialstaat für alle gewährleistet wurden, traten diese an die Stelle traditioneller Netzwerke der Unterstützung aus Versorgungsgründen motivierte soziale Beziehungen werden gesamtgesellschaftlich rückläufig. Dementsprechend nehmen hier Leistungen der informellen Wohlfahrtsproduktion in der Familie tendenziell ab (J. Schmid 2012). Die Rückbesinnung auf die Bedarfsgemeinschaft als Wirtschaftsgemeinschaft, indem sie als sozial absicherndes Netzwerk fungiert, weist jedoch in die entgegengesetzte Richtung. Weiter widersprechen die Mobilitäts- und Flexibilitätsanforderungen den weiteren Rahmenbedingungen von Familienbeziehungen.

Zusammengefasst formt sich folgender faktischer Widerspruch zu Axiomen der Sinnwelt des Aktivierungsdiskurses: Während die Betonung von eigenverantwortlichen und selbstständigen Individuen die postmoderne Lebens- und Erwirtschaftungsform der Individualisierung adaptiert, wird gleichzeitig auf ein gegenläufiges traditionales Familienbild rekurriert, indem die Familienmitglieder eine Wirtschaftsgemeinschaft bilden.

34 Der BRH-Bericht mahnt an, dass bei zusammenlebenden Verwandten die gesetzliche Vermutung bestehe, der Hilfebedürftige beziehe einen Verwandtenunterhalt. Dies sei in den untersuchten Fällen unzureichend geprüft worden (BRH 2006: 24). 


\subsubsection{Aktivierende Arbeitsmarktpolitik versus subventionierter Arbeitsmarkt}

Obwohl die Annahme einer (nicht näher definierten) Vollbeschäftigung ${ }^{35} \mathrm{im} \mathrm{Ak-}$ tivierungsdiskurs dominiert, wird auch eine gegenläufige Entwicklung registriert: »Intensiver Unterstützung « zum Trotz werden nicht alle Arbeitslosen in den Arbeitsmarkt vermittelt (BA 2006: 66). Der Geschäftsführer der ARGE Düsseldorf erklärte 2006 im SGB-II-Jahresbericht, dass einige Erwerbslose die wheutigen Anforderungen des Arbeitsmarktes nicht in vollem Umfang erfüllen und im Wettbewerb um freie Arbeitsplätze nicht bestehen können.« (BA 2006: 66) Zusätzliche Beschäftigungsmöglichkeiten seien notwendig - der bereits angesprochene Übergangsarbeitsmarkt dürfte hier gemeint sein, eventuell aber auch ein staatlich geförderter Arbeitsmarkt, den der Aktivierungsdiskurs durch die Beschäftigungsförderungsmaßnahmen zwar zwingend etabliert, jedoch zugleich kategorisch ausschließt.

»Auch wenn in den einschlägigen Bestimmungen immer wieder darauf verwiesen wird, dass diese Maßnahmen die Arbeitslosen dazu befähigen sollen, in den ersten Arbeitsmarkt zu gelangen, stellen sie selbst doch einen eigenen, einen zweiten Arbeitsmarkt dar (vgl. Friedrich/Wiedemeyer 1998: 311 ff.).«(Kantel 2002b: 127)

Nun stellt sich die Frage, was mit denjenigen geschieht, die eventuell nie wieder eine Erwerbsarbeit aufnehmen können? Kantel konstatiert für das alte BSHG und Job-AQTIV-Gesetz: Statt einem vom damaligen Kanzler Schröder kritisierten »Recht auf Faulheit« nehmen mittels repressiver Instrumentarien der Arbeitsförderung die finanziellen Leistungen des Arbeitsamtes immer weiter ab. Gleichzeitig wird die Akzeptanz der Forderung zunehmen, jede Arbeit anzunehmen je länger die Arbeitslosigkeit dauert (Kantel 2002a). Genau diese von Kantel erwartete Entwicklung bestätigt sich in den hier analysierten Texten.

Die Gesellschaftsintegration wird im Aktivierungsdiskurs also als Integration in eine Erwerbsposition definiert, die nicht notwendig ausbildungsadäquat und die Lebenshaltungskosten deckend sein muss. Gleichzeitig wird die Problemlage

35 Ökonomen sprechen ab einer Arbeitslosenquote von drei bis fünf Prozent von Vollbeschäftigung. Das würde der angelsächsischen Beschreibung einer Vollbeschäftigung entsprechen und die Annahme stützen, dass »jeder, der einen Job will, einen findet«. Der Aktivierungsdiskurs negiert jedoch einen subventionierten Arbeitsmarkt auf Dauer und fordert gleichzeitig Beschäftigung für alle Staatsbürger im ersten Arbeitsmarkt. 
forciert individualisiert: Das Individuum steht unter dem Druck zur Aufnahme einer Erwerbstätigkeit trotz beziehungsweise gerade wegen eines strukturellen Mangels an unbefristeten Vollzeitstellen. Die Kriterien der autonomen Entscheidung für eine Tätigkeit und autonome Selbstversorgung können nur noch eingeschränkt angelegt werden, stehen jedoch unter dem Vorzeichen einer verstärkten Individualverantwortung. Im SGB-II-Jahresbericht für 2007 wird festgestellt, dass die Zahl der erwerbsfähigen Hilfebedürftigen nicht deutlich verringert werden konnte, sich allerdings die Zusammensetzung in erfreulicher Weise verändert habe: So seien mehr Personen geförderte Maßnahmenteilnehmende und Leistungsempfangende mit Erwerbseinkommen (BA 2008a: 17). Diese Entwicklung hat sich fortgesetzt, da nur zwei von fünf Arbeitslosengeld-II-Beziehenden arbeitslos sind (BA 2013e: 10). Auf individueller Ebene sei Letzteres positiv zu bewerten, denn »Erwerbstätigkeit mit SGB-II-Leistungsbezug ist besser als gar keine Arbeit.« (BA 2008a: 18) Auf der Ebene des SGB II habe die steigende Erwerbstätigkeit zur Ausgabensenkung für passive Leistungen bereits 2008 beigetragen (BA 2008a: 19). Gesunkene passive Leistungen von über 12 Prozent im Jahr 2012 im Vergleich zum Vorjahr werden ebenfalls auf eine günstige Arbeitsmarktentwicklung zurückgeführt (BA 2013e: 39f.). Demnach führt das Ziel der Integration in Erwerbsarbeit nicht gleichzeitig aus der Hilfebedürftigkeit heraus. Eine weitere damit verbundene Problematik in der Diskurslogik ist die Nachhaltigkeit der Erwerbsintegration. Es gelinge zwar schneller, erwerbsfähige Hilfebedürftige von staatlicher Unterstützung unabhängig zu machen, doch sei es bei vielen nicht möglich, den Leistungsbezug dauerhaft zu beenden. ${ }^{36}$

Die partielle Erwerbsintegration wird von einer erzwungenen Selbststigmatisierung der Arbeitslosen begleitet, die letztlich zur (Re-)Produktion von >undeserving poor führt. So fördert die positive Diskriminierung von Personen in sozialen Problemlagen eine Reaktivierung der Logik der herkömmlichen Fürsorge, die im Arbeitsrecht überwunden werden sollte: Wenn Hilfebedürftige unterstützt werden wollen, müssen sie ihre Unfähigkeit nachweisen und eine Benachteiligung hinsichtlich des >normalen` Arbeitsmarkts aufweisen. Mit solchen Feststel-

36 Mehr als die Hälfte der im Zeitraum von Dezember 2011 bis November 2012 zugegangenen erwerbsfähigen Leistungsberechtigten hat in den vorherigen zwölf Monaten bereits mindestens einmal die Grundsicherung erhalten (BA 2013e: 11). Ein ähnliches Bild ergab sich 2007, damals wurde die hohe Zahl der »Rückkehrer« als Ergebnis kurzzeitiger Einkommen vermutet: etwa Unterhaltszahlungen, die die Hilfebedürftigkeit unterbrechen, befristete Arbeitsverhältnisse und Scheitern der Arbeitsverhältnisse in der Probezeit (BA 2008a: 15f.). 
lungen der Nicht-Integrierbarkeit und Schwäche werden die sozialen Verhältnisse zementiert (Castel 2000: 411). So zeigt sich die komplexe Aufgabe der aktivierenden Arbeitsmarktpolitik, die einerseits Menschen hin zur Erwerbstätigkeit aktivieren möchte und andererseits durch die Anwendung von Beschäftigungsförderungsinstrumenten auch Personen stigmatisiert. Weiter beinhaltet ein arbeitsmarktnahes Agieren wenige niederschwellige Arbeitsmarktinstrumente vorzuhalten und damit die Bedeutung sozialer Aktivierung nicht wahrzunehmen.

»Der Sinn der Eingliederungspolitik lässt sich vielleicht folgendermaßen zusammenfassen: Er besteht darin, sich um die durch die Konjunktur arbeitsunfähig gewordenen Arbeitsfähigen zu kümmern. Darin besteht ihre Originalität im Vergleich zur klassischen Sozialhilfepolitik, die auf ein Defizit bei ihrer Klientel zugeschnitten ist, sowie zur Integrationspolitik, die sich unterschiedslos an alle wendet. Sie bewegt sich in diesen besonders verwundbaren Zonen des Gesellschaftslebens, in die sich die >nutzlosen Normalen abgesetzt haben oder gerade im Begriff sind, es zu tun.« (Castel 2000: 378)

Insgesamt zeigen die hybriden Momente im Aktivierungsdiskurs (Kapitel 4.5), dass widersprüchliche Annahmen zu Axiomen des Aktivierungsdiskurses verklammert werden, statt eine Neuorientierungen der konträren Annahmen zu bewirken. Während die Integration in Gesellschaft über die Familie weiter besteht, jedoch die Funktionen der Wirtschaftsgemeinschaft in der Postmoderne aufgeweicht werden, wird auf die Versorgung durch Familie und Partnerschaften appelliert. Weiter wird das Inklusionsprinzip des Normalarbeitsverhältnisses geschmälert, denn es zeigt sich, dass trotz einer wachsenden Beschäftigung bei einem steigenden Teil der Personen eine dauerhafte zum Teil phasenweise Hilfsbedürftigkeit von Transferleistungen bestehen bleibt. Das Erwerbsprinzip des Aktivierungsdiskurses führt insofern nicht zwingend zu einer umfassenden Selbstversorgung der Erwerbstätigen. Vor diesem Hintergrund erscheint eine Neujustierung der Arbeitsbedingungen sinnvoll sowie der besondere Fokus auf eine arbeitsmarktferne Klientel, um zu eruieren, welche Instrumente der Beschäftigungsförderung hier die soziale Teilhabe fördern, um eine Zementierung der Nicht-Integrierbarkeit dieser arbeitsmarktfernen Individuen zu verhindern.

\subsection{DisKURSTRAgENDE INSTITUTIONEN IN DER ARBEITSVERWALTUNG}

Der Aktivierungsdiskurs wird über Institutionen der Arbeitsverwaltung ausgeführt und (re-)produziert, deren Rolle in den Arbeitsmarktreformen neu definiert 
wurde. Da insbesondere die zentrale Vorgängerinstitution (Bundesanstalt für Arbeit) in der Öffentlichkeit und aus Sicht des Diskurses versagt hat, sollte eine Restrukturierung neue Bedingungen und neue Instrumentarien für die Verwaltung von und das Vorgehen gegen Arbeitslosigkeit bieten. »Markt und Staat bzw. öffentliche Verwaltung entwickeln sich mehr und mehr zu verknüpften Ordnungssystemen. « (Schedler/Proeller 2011) So sollen nach der Arbeitsmarktreform Marktmechanismen in der Arbeitsverwaltung Effizienz und Effektivität steigern sowie eine höhere Zufriedenheit bei den Erwerbslosen bewirken. Im Folgenden werden Eckpunkte dieses gewandelten Selbstverständnisses dargestellt: das Selbstbild als dynamische Verwaltung (Kapitel 4.6.1) sowie der als inhaltlicher Motor der Aktivierung fungierende Grundsatz »Fördern und Fordern« (Kapitel 4.6.2). Weiter werden die AkteurInnen der Beschäftigungsförderung skizziert (Kapitel 4.6.3), die im Rahmen der Entstehung von Maßnahmen Sozialer Aktivierung von Bedeutung sind.

\subsubsection{Dynamische Verwaltung in gelebten Organisationen}

Max Weber beschrieb in Wirtschaft und Gesellschaft 1921/22 die modernen Strukturen von Wirtschaft und Verwaltung, mit einem bürokratischen Verwaltungsstab der legalen Herrschaft im Idealtypus der Rationalen Herrschaft (Weber 2005): gesetzte Regeln und die Exekutive durch einen Verwaltungsapparat mit fachlich ausgebildetem Personal schaffen eine Legitimation der Ordnung über eine durch Rechtssicherheit und kommunizierbare Normen ausgeübte legale Herrschaft. ${ }^{37}$ Die New Public Management-Theorien der 1980er Jahre orientierten sich an diesem idealtypischen Ansatz, als sie die Verwaltungspraktiken weiterentwickelten, verbanden ihn jedoch grundlegend mit marktwirtschaftlichen Mechanismen und Denkweisen. ${ }^{38}$

Die Bundesagentur für Arbeit und lokale SGB-III- und SGB-II-Träger sollen Arbeitslose möglichst zeitnah in den ersten Arbeitsmarkt respektive in Beschäftigungsförderungsmaßnahmen integrieren. Es wird angenommen, dass sie diese Aufgabe umso effektiver bewältigen, je wirtschaftlicher sie handeln, indem sie sich zunehmend an unternehmerische Organisationsstrukturen anpassen (HartzKommission 2002: 13ff.). Die Bundesagentur für Arbeit, als Nachfolgeinstituti-

37 Vergleiche Kapitel 3.3.2 Die Arbeitsverwaltung als Schnittpunkt von Machtdiskurs und Subjektivität.

38 Aus der umfangreichen Literatur seien herausgegriffen: (Budäus 1994) sowie (Schedler/Proeller 2011). 
on der Bundesanstalt für Arbeit, wird so zu einem »Dienstleister mit privatwirtschaftlichen Führungsstrukturen« (Hartz-Kommission 2002: 13). Auch Konkurrenz unter den lokalen SGB-II-Trägern um das best practice-Modell ist erwünscht. Dabei besteht eine Aufgabenteilung zwischen den verschiedenen Instanzen (Hartz-Kommission 2002: 43): Während der Bundesagentur für Arbeit die Funktion zentraler Steuerung zukommt, sind lokale Einheiten (JobCenter laut dem Bericht der Hartz-Kommission) weitgehend eigenverantwortlich. JobCenter sollten Anlaufstelle für alle Leistungsberechtigten sein - »Hilfe aus einer Hand « (BMWA (ehemaliges), 2005a: 34) gewähren und »kundenorientiert« arbeiten. ${ }^{39}$ Allerdings wurde auf der verwaltungshorizontal niedrigsten Stufe nicht eine Institution geschaffen, sondern Arbeitsgemeinschaften/Jobcenter für den SGB-II-Bereich sowie Agenturen für Arbeit für den SGB-III-Bereich. Für Sozialhilfe-Leistungen nach dem SGB XII sind weiterhin die Kommunen zuständig. Statt der Verantwortungsteilung von Kommunalverwaltungen und Bundesagentur für Arbeit wurde eher eine Verantwortungsauslagerung von der Bundesagentur durchgeführt, mit der geschäftspolitischen Strategie einer Fokussierung auf Versicherungskunden (Sell 2006: 143).

Trotz dieser administrativen Teilung möchte die Bundesagentur über Rechtsvereinfachungsvorschläge mehr Transparenz, Akzeptanz und Kundenzufriedenheit herstellen, indem Bürokratie abgebaut wird (BA 2013e: 34). Die vermittlungsunterstützenden Leistungen und Fördermaßnahmen sollten mit dem Gesetz zur Neuausrichtung der arbeitsmarktpolitischen Instrumente Ende 2008 vereinfacht werden. Auch eine Ausrichtung auf die Kundenzufriedenheit zeigt die ab 2012 durchzuführende Qualitätssicherung über einen Index aus der Kundenzufriedenheit und der Prozessqualität, also der Rechtmäßigkeit, Wirksamkeit und Wirtschaftlichkeit der Aufgabenerledigung (BA 2013e: 20). Damit reagiert die BA auch im Interesse der Leistungsbeziehenden auf Untersuchungen, die beispielsweise ergaben, dass nur etwa die Hälfte der Teilnehmenden Aktivierungsmaßnahmen als Orientierungshilfen einschätzt und nur jede/r Vierte von einer unterstützenden Wirkung bei der Stellensuche berichtet (Schütz et al. 2011: 303). So ist es ein Anliegen, dass das "Vertrauen in die Arbeit der Jobcenter» zunimmt und jeder zweite Befragte »eine gute Meinung von seinem Jobcenter« hat (BA 2013e: 23). Ferner wird das Personal der SGB-II-Träger im Aktivierungsdiskurs nun als Erfolgsfaktor der Dienstleistungsunternehmen begriffen. Für eine effektive Bewältigung der Arbeit sei die Identifikation der Fachkräfte mit ihren Aufgaben unerlässlich, genauso wie die Zufriedenheit mit Arbeitsbe-

39 Vergleiche Kapitel 4.2.1 Arbeitsmarktdienstleistungen für Kundinnen und Kunden. 
dingungen und -umfeld in konstruktiv-kritischer Weise (Hartz-Kommission 2002: 178). Die Subjektivierung der Arbeit ist auch in dieser »gelebten Organisation« (Hartz-Kommission 2002: 178) Teil des Diskurses.

Kontrolliert wird der Erfolg des zentralen Arbeitsmarktdienstleisters über sogenannte Zielvereinbarungen: Sie beinhalten das angestrebte Ergebnis der Leistungserbringung und in ihr werden jährlich allgemeine Ziele festgelegt, die zwischen dem Bundesministerium für Arbeit und Soziales und der Bundesagentur für Arbeit im Einvernehmen mit dem Bundesfinanzministerium ausgehandelt werden ( $\$ 48$ Abs. 1 SGB II). Auf lokaler Ebene werden diese für den SGB-IIBereich durch Vereinbarungen zwischen der Trägerversammlung ${ }^{40}$ und der Geschäftsführung der SGB-II-Träger ergänzt. Diese beinhalten für die Qualitätssicherung genutzte Zieldefinitionen insbesondere zur Integrationsquote der zu aktivierenden Klientel und sind für die Maßnahmenplanung und Genese Sozialer Aktivierung von großer Bedeutung. Die allgemeine Zieldefinition der quantifizierbaren Vermittlungsquoten wird lokal in den Kontext der Arbeitsmarktstruktur und der Beschäftigungsförderung der vergangenen Jahre gestellt.

Auch eine regionale Verzahnung der Wirtschafts- und Arbeitsmarktpolitik wird angestrebt. Nach $\S 18$ SGB II sollen Agenturen für Arbeit und SGB-II-Träger durch örtliche Zusammenarbeit mit lokalen AkteurInnen (etwa Kommunen, Trägern der freien Wohlfahrtspflege, Vertretern der Arbeitgeber und -nehmer) das Angebot der Eingliederungsleistungen in Arbeit optimieren und Leistungsmissbrauch verhindern.

So wurden die SGB-II-Träger insofern an ökonomische Strukturen angepasst, als sie sich als »moderner Arbeitsmarktdienstleister« »auf die Kernbereiche der Arbeits- und Ausbildungsvermittlung, Auszahlung von Lohnersatzleistungen und aktive Arbeitsmarktpolitik konzentrieren« (Hartz-Kommission 2002: 15) sollen.

»Der Sozialstaat von heute ist immer weniger als simples Transfersystem zu begreifen. Nirgends zeigt sich dies so deutlich wie im Bereich der institutionellen Bearbeitung von Erwerbslosigkeit. Die Arbeits- und Beschäftigungsförderung ist ein Paradebeispiel für einen Paradigmenwechsel wenn nicht der modernen Sozialpolitik - die schon immer, um mit Franz-Xaver Kaufmann (2002: 96-104) zu sprechen, ökologische und sozialpädagogische Interventionsformen umfasste, so doch in der mit ihr befassten (Fach-)Öffentlichkeit: zu offenkundig ist der Trend weg von der Interpretation des Sozialstaats als >Geldumver-

40 Die Trägerversammlung besteht aus Vertreterinnen und Vertretern der Agentur für Arbeit und des kommunalen Trägers (nach $\S 44 \mathrm{c}$ SGB II). 
teilungsmaschine hin zur Betonung der in ihm ausgebildeten Elemente dienstleistungsbasierter Wohlfahrtsproduktion.« (Bode 2011: 317)

Instrumente der Mittelallokation und der Lenkung, die aus New Public Management-Theorien in die Arbeitsverwaltung überführt werden und letztlich aus der Betriebswirtschaft stammen, sind ausgerichtet an einer ergebnisorientierten Steuerung über quantifizierbare Ziele, (quasi-)wettbewerbliche Leistungserbringung sowie auf eine Erfolgsbewertung auf Basis (kurzfristig) zählbarer Outputs (Bode 2011: 319). Einerseits zielt die Verwaltungsorganisation durch die Instrumente des New Public Managements auf mehr Effizienz und Effektivität. Hierzu dienen aus Sicht der Initiatoren insbesondere das Neue Steuerungsmodell mittels Kundenorientierung sowie eine verstärkte Ergebniskontrolle der Arbeitsverwaltung, »die sich bereits in den Eingliederungsbilanzen des $\S 11$ SGB III abgezeichnet hat und die nun durch »leistungsorientierte Führung«, »übergreifendes Controlling« und »Innenrevision« (BA 1999, S.17f.) einen weiteren Grundgedanken des Neuen Steuerungsmodells aufgreift« (Schönig 2002: 10): die freie Förderung, die Profiling-Methodik bezüglich der Leistungsbeziehenden sowie die Kooperation mit den Trägern der Kommunalen Wirtschafts- und Sozialpolitik (Schönig 2002). Andererseits ermöglicht eine Eingriffs- und Durchgriffsverwaltung seitens des Bundes gegenüber der BA wie auch seitens der Zentrale und der Regionaldirektionen gegenüber den Arbeitsagenturen vor Ort nicht die notwendige Voraussetzung für eine wirkungsorientierte Zielsteuerung mit operativen Freiheitsgraden (Sell 2006: 140).

Zusammengefasst ermöglicht die bürokratische Strukturierung der Arbeitsverwaltung Rechtssicherheit und kommunizierbare Normen. Zugleich wird die Arbeitsverwaltung als Dienstleister mit privatwirtschaftlichen Führungsstrukturen verstanden, der durch eine ergebnisorientierte Steuerung quantifizierbare Ziele neben der Erwerbsintegration etwa Akzeptanz und Kundenzufriedenheit - herstellen soll. Doch die Top-down-Steuerung der jeweils hierarchisch höheren Institutionen $^{41}$ schafft nicht die notwendigen Voraussetzungen für eine wirkungsorientierte Zielsteuerung mit operativen Freiheitsgraden. Entsprechend sind die diskurstragenden Institutionen nicht uneingeschränkt handlungsfähig - finanzielle Barrieren ${ }^{42}$ prägen ihr Wirken: So ist die im folgenden Kapitel 4.6.2 angeführ-

41 Seitens des Bundes gegenüber der Bundesagentur für Arbeit, seitens der Zentrale und der Regionaldirektionen gegenüber den SGB III- und SGB II-Trägern.

42 Vergleiche Kapitel 5.2.1 Planung und finanzielle Mittel. 
te Zielvereinbarung an betriebswirtschaftlichem Kalkül (insbesondere der Haushaltslage) orientiert und wirkt sich auf die in der Beratung zur Verfügung stehenden Arbeitsmarktdienstleistungen aus. Somit hat die effektive und ökonomische Wirtschaftsweise, die sich an der Erwerbsintegration orientiert auch einen Einfluss auf die individuelle Förderung der Leistungsbeziehenden.

\subsection{2 »Fördern und Fordern« als Motor der Aktivierung}

Die explizit betonte Ausrichtung auf die Kundenorientierung (Hartz-Kommission 2002) sowie die Öffnung der Verwaltung gegenüber den Bedürfnissen und Anliegen der Bürgerinnen und Bürger, demonstrieren sicherlich den Wunsch nach einer Umsetzung der »Vision von der menschlichen Verwaltung « (Schedler/Proeller 2011). ${ }^{43}$ Auch die Dienstleistungsziele wie »Kundenservice« und »Kundenzufriedenheit« (Hartz-Kommission 2002: 58) dokumentieren dies. Leistungsbeziehende sollen jedoch auch eigeninitiativ und selbstständig an der Erwerbsintegration mitwirken. ${ }^{44}$ Den Antrieb zur Eigeninitiative soll dabei der mit der Gesetzesänderung im SGB II verankerte Grundsatz des »Förderns und Forderns « bieten. Dieser verpflichtet den Leistungsbeziehenden, »aktiv an allen Maßnahmen zu seiner Eingliederung in Arbeit mitzuwirken, insbesondere eine Eingliederungsvereinbarung abzuschließen.« (§ 2 Abs. 1 SGB II Grundsatz des Forderns) Das gesamte Maßnahmenpaket zielt darauf ab, die Sozialstaatlichkeit vom bisherigen alimentierenden Modell zum aktivierenden Staat umzuwandeln. Hilfe zur Selbsthilfe (Hartz-Kommission 2002: 97), aber auch Fördern und Fordern drücken dies aus:

»Der Grundsatz `Fördern und Fordern〈 sowie `keine Leistung ohne Gegenleistung`erhält im Sinne der Neuausrichtung der Arbeitsförderungspolitik zu einer aktivierenden Arbeitsmarktpolitik eine neue Akzentuierung, die im Begriff `Eigenaktivitäten auslösen - Sicherheit einlösen` zum Ausdruck kommt. `Fördern und Fordern` entspricht der Erwartungshaltung des Versicherers an den Versicherten, den materiellen und nicht-materiellen Leistungen des Arbeitsamtes [dies lässt sich auch auf den SGB-II-Bereich übertragen; Anm. CF] im Sinne der Schadensminderungspflicht durch ein angemessenes, zielführendes Verhalten zu begegnen (Bewerbungen, Angebote zu Vorstellungsgesprächen annehmen, tatsächliche Teilnahme an Maßnahmen, Annahme zumutbarer Stellenangebote). `Ei-

43 Vergleiche Kapitel 4.6.1 Dynamische Verwaltung in gelebten Organisationen.

44 Vergleiche Kapitel 4.2 »Arbeitslosigkeit >bekommt ein Gesicht«: Aktivieren eines erweiterten Personenkreises. 
genaktivitäten auslösen - Sicherheit einlösen` beinhaltet demgegenüber ein aktivierendes Angebot und Versprechen« (Hartz-Kommission 2002: 45).

Mit den SGB II-Leistungsbeziehenden wird ein Eingliederungsplan aufgestellt. In einer beiderseits verbindlichen Zielvereinbarung - der Eingliederungsvereinbarung $(\mathrm{EV})^{45}$ - verpflichtet sich der Leistungsbeziehende auf konkrete Schritte zur Vorbereitung einer Erwerbsaufnahme. Bei Verstoß gegen die Eingliederungsvereinbarung kommt es zu Sanktionen in Form von Sperrzeiten. Auf eine Weigerung der Unterzeichnung können 30 Prozent der Leistungen gekürzt werden (§ 31 Abs. 1 Nr.1a SGB II). Die Sanktionspraxis ist dabei abhängig von den Steuerungsvorgaben des jeweiligen SGB-II-Trägers (BMAS 2008) sowie von den jeweiligen Vermittlern (Ludwig-Mayerhofer et al. 2009, Ames 2008: 158ff.). So wird teilweise möglichst lange auf den Einsatz der verfügbaren Sanktionsmittel verzichtet (Ames 2008: 159, Baethge-Kinsky et al. 2007: 51f.). ${ }^{46}$ Die Eingliederungsvereinbarung hat einen ambivalenten Charakter: Sie ermöglicht in einem durch Abhängigkeit und Zwang gekennzeichneten Verhältnis zwischen erwerbsfähigen Hilfebedürftigen und Verwaltung, den SGB-II-Leistungsbeziehenden zu aktivieren und zur Kooperation zu bewegen, um die Arbeitsmarktintegration zu erreichen. Denn einerseits dient sie der rechtlichen Fixierung der zu unternehmenden Schritte der SGB-II-Leistungsbeziehenden. Sie wird in der Praxis von den SGB-II-Träger-Beschäftigten teilweise nicht eindeutig als rechtlichbindend dargestellt (Schütz et al. 2011: 145f.) oder nur allgemein mit dem Ziel der Arbeitsaufnahme erläutert (Schütz et al. 2011: 139). Andererseits bietet die Eingliederungsvereinbarung die - wenn bislang noch nicht ausschöpfend genutzte - Möglichkeit, das asymmetrische Verhältnis durch eine beidseitige Zieldefinition und -fixierung zu mildern und somit sozialen Rechten der Erwerbslosen eine Ausübungsform zu bieten (Bieback 2009). Dies wäre insbesondere der Fall, wenn eine gemeinsame Ausarbeitung der Zieldefinition zwischen SGB-II-Leistungsbeziehenden und SGB-II-Träger-Beschäftigten geschehen würde, da diese die individuelle Autonomie der SGB-II-Leistungsbeziehenden stärke. Doch die Praxis gestaltet sich meist als asymmetrische Gesprächssituation, in der die

45 Dagegen besteht im SGB-III-Bereich keine Notwendigkeit der Eingliederungsvereinbarung ( $\S 6$ Abs. 1 S. 2, 37 Abs. 2 SGB III) (Bieback 2009: 191) hier kann auch ein Verwaltungsakt angelegt werden.

46 Ebenso existiert sowohl bei den Fachkräften (Ames 2008: 130ff.) als auch in der Begleitevaluation (Eppel et al. 2012: 51f., 75, Hofmann et al. 2011) die Auffassung, dass Sanktionen die Aktivierung maßgeblich unterstützen. 
SGB-II-Träger-Fachkräfte dominieren (Schütz et al. 2011: 161). Trotzdem hat die Eingliederungsvereinbarung eine Konkretisierungs- und Sicherungsfunktion, da Rechte und Pflichten offen kommunizier- und kalkulierbar werden, insbesondere wenn sich im >schlüssigen Vermittlungskonzept` die Verhältnismäßigkeit (Erforderlichkeit und Geeignetheit) der Pflichten des SGB-II-Leistungsbeziehenden messen lassen (Bieback 2009). ${ }^{47}$ Eine gleichberechtigte Kommunikation ist zwischen Bürgern und Verwaltung jedoch nicht möglich (Promberger 2009). Auch erschwert die Informationsdichte der Verschränkung von Rechten, Pflichten und Rechtsfolgebelehrung das Verstehen der Eingliederungsvereinbarung (Schütz et al. 2011: 300f.), wodurch es nicht verwundert, dass nur ein Drittel von 41 befragten SGB-II-Leistungsbeziehenden bestätigt, dass die Eingliederungsvereinbarung hinsichtlich der eingegangenen Verpflichtungen und der zu tragenden Konsequenzen verstanden wurden (Schütz et al. 2011: 302).

Der Grundsatz des Förderns gemäß § 14 SGB II wird auf Seiten der Verwaltung dadurch gewährleistet, dass ein Ansprechpartner bei der Eingliederung in Arbeit unterstützen muss. Die »intensive Betreuung« (Hartz-Kommission 2002: 49), ${ }^{48}$ die als Kernstück zur Reintegration von Menschen ohne Gelderwerb gilt, wird durch persönliche Ansprechpartner ( $\S 4$ Abs. 1 Nr.1 und 14 Abs. 2 SGB II), Arbeitsvermittler oder Fallmanager geleistet und nicht nur im Diskurs, sondern auch gesetzlich normiert.

Der Integrationsprozess umfasst im SGB-II-Bereich in der Regel drei Aspekte: 1.) den Erstkontakt (Anliegensklärung, Kundensteuerung und Erstberatung), 2.) die Betreuung durch eine Integrationsfachkraft und 3.) im Idealfall eine erfolgreiche Integration (Schütz et al. 2011). Personen, die die Versicherungs- und Vermittlungsleistungen in Anspruch nehmen wollen, werden in der Regel zunächst im Rahmen eines längeren Erstgespräches auf ihre Bedarfe und Fähigkeitsfelder hin analysiert. Die Kategorisierung heterogener Personengruppen und eine damit einhergehende Klassifizierung von Maßnahmenmöglichkeiten sollen den Vermittlungsprozess handhabbar gestalten. Weiter soll sie die Effizienz der arbeitsmarktpolitischen Ressourcen garantieren und Fehlallokationen von Mitteln vermeiden. Dabei werden die SGB-II-Leistungsbeziehenden in spe-

47 Die Leistungen sollten jedoch die gesetzliche Verpflichtung zu Beratung und Vermittlung übersteigen.

48 Rechtlich als umfassende Unterstützung bei der Eingliederung in Arbeit definiert ( $\$ 14$ SGB II). 
zifische Zielgruppen klassifiziert, abhängig von ihrer Nähe ${ }^{49}$ zum Arbeitsmarkt. Diesen Personengruppen gehören je unterschiedliche Portfolios an Dienstleistungen und Intensitäten der Beratungsgespräche an. Die Zuschreibung erfolgt im sogenannten Profiling, in dem »neben den harten Kriterien (zum Beispiel Fakten zur Jobhistorie) insbesondere auch weiche Faktoren (zum Beispiel Motivation, Teamfähigkeit oder Flexibilität)« (Hartz-Kommission 2002: 71) berücksichtigt werden sollen. Die Kundenkategorisierungen wurden 2009 überarbeitet. ${ }^{50}$ Zuvor sollen marktnahen Informationskunden vorwiegend Selbstinformationseinrichtungen zur Verfügung gestellt, Beratungskunden passgenaue Angebote durch Vermittler unterbreitet und marktfernen, häufig langzeitarbeitslosen Betreuungskunden von speziell ausgebildeten Fallmanagern ein Eingliederungsplan erstellt werden (Hartz-Kommission 2002). Angestrebt wird damit insbesondere die Betreuungskunden, also die marktfernste Personengruppe, intensiver zu betreuen (BMAS 2002: 49). Doch hat die Kundendifferenzierung in der Verwaltungspraxis einen geradezu gegenteiligen Effekt einer Dienstleistungsunterversorgung der SGB-II-Leistungsbeziehenden mit dem höchsten Unterstützungs- und Beratungsbedarf, denn die Personen haben eine niedrigere Beratungszeit und Maßnahmenfrequenz (Behrend 2007). Ohnehin chancenreiche Marktkunden werden häufig privilegiert und soziale Ungleichheit in der Arbeitsverwaltung damit reproduziert (Ludwig-Mayerhofer/Sondermann 2012). Mit dem 4-Phasen-Modell der Integrationsarbeit ab 2009 sollen die Transferleistungsbeziehenden stärkenorientiert von den umsetzenden SGB-II-Trägern ${ }^{51}$ kategorisiert und sich ebenso das weitere Vorgehen der SGB-II-Träger-Fachkräfte entlang der jeweiligen Kategorien ausrichten (Oschmiansky 2011). ${ }^{52}$ In der ersten, der vier Phasen ${ }^{53}$ wird das Profiling durchgeführt.

49 Darunter versteht der Diskurs die Integrationswahrscheinlichkeit in den ersten Arbeitsmarkt, wie sie die jeweils zuständigen Agentur- beziehungsweise Jobcenterbeschäftigten definieren.

50 Bis zum Sommer 2009 wurde folgende Kategorisierung vorgenommen: Integrationsfern: Betreuungs- und Hilfebedarf (IF); Stabilisierungsbedarf: Heranführen an eine Erwerbstätigkeit; Förderbedarf: Heranführen an den ersten Arbeitsmarkt (IG); Integrationsfähig: Integration in den ersten Arbeitsmarkt (IN); Integriert, aber weiterhin hilfebedürftig (I) (Schütz et al. 2011: 36).

51 Sowie den SGB III-Trägern, da die Kategorisierung für beide Arbeitsverwaltungen gilt.

52 Die Kontakthäufigkeit zu den SGB II-Leistungsbeziehenden sowie das zur Verfügung stehende Portfolio an Dienstleistungs- und Vermittlungsangeboten orientiert sich da- 
Tabelle 2: Kundengruppen im 4-Phasen-Modell

\begin{tabular}{|l|l|}
\hline Profile & Beschreibung \\
\hline Marktprofile & $\begin{array}{l}\text { Personen ohne vermittlungsrelevante Handlungsbe- } \\
\text { darfe; bei diesen Personen ist eine Integration in den } \\
\text { Arbeitsmarkt innerhalb von sechs Monaten wahr- } \\
\text { scheinlich }\end{array}$ \\
\hline Aktivierungsprofile & $\begin{array}{l}\text { Personen mit vermittlungsrelevanten Handlungsbe- } \\
\text { darfen in der Kategorie »Motivation« }\end{array}$ \\
\hline Förderprofile & $\begin{array}{l}\text { Personen mit vermittlungsrelevanten Handlungsbe- } \\
\text { darfen in einer der drei Kategorien »Qualifikation«, } \\
\text { »Leistungsfähigkeit« oder »Rahmenbedingungen«; } \\
\text { diesen Personen soll in erster Linie eine Weiterbil- } \\
\text { dungsmaßnahme angeboten werden }\end{array}$ \\
\hline Entwicklungsprofile & $\begin{array}{l}\text { Personen mit Handlungsbedarfen in einer der Kate- } \\
\text { gorien der Förderprofile sowie einer weiteren Di- } \\
\text { mension }\end{array}$ \\
\hline Stabilisierungsprofile & $\begin{array}{l}\text { Personen mit Handlungsbedarfen in der Dimension } \\
\text { »eistungsfähigkeit« sowie 2 weiteren }\end{array}$ \\
\hline Unterstützungsprofile & $\begin{array}{l}\text { Personen mit Handlungsbedarfen in der Dimension } \\
\text { »Rahmenbedingungen« sowie in zwei weiteren } \\
\text { Schlüsselgruppen }\end{array}$ \\
\hline
\end{tabular}

Quelle: Eigene Darstellung nach (Oschmiansky 2011)

In der Praxis wird die Zielsetzung der Erwerbsintegration von Leistungsbeziehenden unter anderem über Unterstützung bei der Arbeitssuche und gezielter

bei an einer Kategorisierung der SGB II-Leistungsbeziehenden, die sich an der Arbeitsmarktnähe orientiert (Hielscher/Ochs 2009: 22-28). Dies waren vor dem 4Phasen-Modell Marktkunden, Beratungskunden Aktivieren, Beratungskunden Fördern, Betreuungskunden.

53 In der zweiten Phase wird eine Zielfestlegung, in der dritten die Wahl der Strategie und in der vierten Phase die Umsetzung vorgenommen (Oschmiansky 2011). 
Vermittlung positiv beeinflusst, insbesondere wenn die SGB-II-Beschäftigten eine geringe Fallbelastung haben und Zeit für eine intensive Kommunikation zur Verfügung steht, um die Bedürfnisse der Erwerbslosen zu erkunden (Eppel et al. 2012, Bieback 2009, Fromm/Sproß 2008, Konle-Seidl/Eichhorst 2008a, Deutscher Bundestag 2008a). Hier kommt es zu unterschiedlichen Bearbeitungsweisen der Lebenssituationen, etwa ob schwierige Ausgangssituationen wie Krankheit, Schulden oder fehlender Ausbildungsabschluss berücksichtigt werden (Kolbe 2012). Der Beratungsprozess selbst ist geprägt durch ein Übergewicht bürokratisch-verwaltender Handlungsmuster gegenüber kundenorientierten Dienstleistungsansätzen (Schütz et al. 2011: 160f.): das Profiling dient in der Praxis nicht als Anknüpfungspunkt für nachvollziehbare Zielbestimmungen, die Fachkräfte dominieren während des gesamten Prozesses ${ }^{54}$ und die Eingliederungsvereinbarung wird nicht interaktiv vereinbart, um als Instrument der Kundeneinbindung zu dienen, sondern häufig bürokratisch oktroyiert. Die thematisierten Anliegen der erwerbslosen Leistungsbeziehenden wurden im Prozess nicht immer wirksam (Schütz et al. 2011: 161). Auch sind Interaktionsprozesse mit jugendlichen Personen seitens der SGB-II-Träger-Beschäftigten zum Teil »sehr direktiv, nur bedingt dialogisch und tendenziell stigmatisierend angelegt « (Schütz et al. 2011: 262). Die Fachkräfte im Fallmanagement und in der Arbeitsvermittlung der SGB-II-Träger haben hier einerseits die Aufgabe der Durchsetzung der Aktivierung mittels Fördern und Fordern und üben damit auch Zwang aus, während sie andererseits mit den Leistungsempfangenden interagieren. Somit muss die Arbeitsverwaltung in der Interaktion mit den Leistungsbeziehenden insbesondere im SGB-II-Bereich die institutionellen und rechtlichen Vorgaben mit den Anforderungen und Wünschen der Leistungsbeziehenden abstimmen. Dass die Sozialverwaltung hier Freiräume nutzt, sowohl um den Interessen der Klientel nachzukommen, als auch die eigene Arbeitsweise zu vereinfachen, ist seit Lipskys Street Level Bureaucracy (Lipsky 1980) bekannt. ${ }^{55}$ Die hier teilweise widerstrei-

54 Dies äußert sich in der Themensetzung und dem Anteil der Redezeit. Es führt in vielen Fällen zu monologischen Kommunikationsstrukturen durch die Verwaltungsfachkräfte.

55 Da die Anforderungen der Leistungsbeziehenden häufig über den beschränkten Ressourcen der Street Level Bürokraten liegen, reagieren diese oftmals mit Manipulationsmöglichkeiten und Interpretationsspielräumen. Diese können einerseits mit einer stärkeren Gewichtung des KlientInneninteresses gegenüber den politischen Vorgaben verbunden sein, jedoch auch andererseits mit der Rationalisierung ihrer Arbeit etwa 
tenden Anforderungen werden insbesondere durch die Aktivierungspolitik verschärft und können bei den Fachkräften zu Handlungsdilemmata (LudwigMayerhofer et al. 2009: 292) führen. Bei den Leistungsbeziehenden können die konträren Anforderungen Krisen hervorrufen, da sie überwiegend eine hohe Erwerbsorientierung haben, jedoch ihre Vorstellung von einer sinnstiftenden Beschäftigung an die Prämissen der Arbeitsverwaltung anpassen müssen (Sondermann 2013).

Insgesamt wird beschrieben, dass sich die diskurstragenden Institutionen in der Arbeitsverwaltung (Kapitel 4.6) für Instrumente des New Public Management öffnen, wobei einerseits eine ergebnisorientierte Steuerung angestrebt wird, jedoch nicht die umfassenden Voraussetzungen für eine wirkungsorientierte Zielsteuerung durch die Top-down-Steuerung zur Verfügung stehen. Weiter dienen Fördern und Fordern als Motor der Aktivierung, indem einerseits über die Eingliederungsvereinbarung und andererseits über die Betreuung der SGB-IITräger-Fachkräfte in einer überwiegend asymmetrischen Beziehung Schritte zur Erwerbsintegration eruiert und festgehalten werden. Die innerinstitutionellen Vorgaben und das Handeln der Fachkräfte sind dabei an der Klassifikation der Leistungsbeziehenden ausgerichtet. Bemerkenswert ist, dass gerade die arbeitsmarktfernen Betreuungskunden in der Praxis durch eine niedrigere Maßnahmenfrequenz und Beratungszeit gekennzeichnet sind, obwohl diesen im Aktivierungsdiskurs eine besonders intensive Förderung zugesprochen wird.

\subsubsection{Die Akteurinnen und Akteure in der Beschäftigungsförderung}

Die AkteurInnen, die in der Beschäftigungsförderung tätig sind, werden hier skizziert. Der für die Maßnahmen Sozialer Aktivierung zentrale Eingliederungstitel, der unter anderem Mittel für Arbeitsmarktdienstleistungen bereithält, ${ }^{56}$ wird über die BA jährlich an die lokalen SGB-II-Träger vergeben. Mit diesen Mitteln können SGB-II-Träger von Maßnahmenträgern Maßnahmenpakete einkaufen

über beschränkte Informationsweitergabe, Verwendung von psychologischem Druck und Klassifizierung sowie Ungleichbehandlung von KlientInnen (Lipsky 1980).

56 Die Grundsicherung für Arbeitsuchende umfasst neben den Leistungen zur Beendigung oder Verringerung der Hilfebedürftigkeit solche zur Sicherung des Lebensunterhalts ( 1 Abs. 3 SGB II). Der Eingliederungstitel umfasst Bundesmittel für die Leistungen zur Eingliederung in Arbeit, die gemäß der Eingliederungsmittelverordnung des BMAS auf die jeweiligen Jobcenter aufgeteilt werden. 
oder die Leistungsempfangenden über Vermittlungsgutscheine selbst ein Angebot aus dem Katalog der Maßnahmenträger auswählen.

\subsubsection{Bundesagentur für Arbeit, Regionaldirektionen und Agenturen für Arbeit}

Die Bundesagentur für Arbeit ${ }^{57}$ ist Trägerin der Arbeitsförderung (§§ 19 SGB I, 1,3 SGB III) und der Grundsicherung für Arbeitsuchende (§ 6 Abs. 1 SGB II).

Sie

»soll dem Entstehen von Arbeitslosigkeit entgegenwirken, die Dauer der Arbeitslosigkeit verkürzen und den Ausgleich von Angebot und Nachfrage auf dem Ausbildungs- und Arbeitsmarkt unterstützen. Dabei ist insbesondere durch die Verbesserung der individuellen Beschäftigungsfähigkeit Langzeitarbeitslosigkeit zu vermeiden.«(§ 1 SGB III)

So zielt die Arbeitsförderung darauf, einen hohen Beschäftigungsstand zu erreichen und die Beschäftigungsstruktur ständig zu verbessern. Mit den §§ 3-5 SGB III wird der Vorrang der Arbeitsvermittlung vor allen anderen Leistungen der Beschäftigungsförderung installiert. Rahmenziele, die das Wirken der Bundesagentur für Arbeit zur Durchführung der Arbeitsförderung leiten sollen, werden mit der Bundesregierung vereinbart (§ 1 SGB III). Insofern können sich die Rahmenziele mit wechselnden bundespolitischen Konstellationen mehr oder minder kurzfristig ändern.

Bereits seit dem Entstehen der Bundesrepublik war die Arbeitsverwaltung Angelegenheit einer bundesunmittelbaren Körperschaft des öffentlichen Rechts mit Selbstverwaltung und dreistufiger Gliederung ( 367 Abs. 1 SGB III). Heutzutage dient der Verwaltungsrat der Bundesagentur für Arbeit als Aufsichts- und Legislativorgan, ${ }^{58}$ er überwacht die Arbeit des hauptamtlichen Vorstands und berät den Vorstand, beschließt den Haushaltsplan und genehmigt den jährlichen Geschäftsbericht (BA 2012a).

Auf der obersten Verwaltungsebene steht die Bundesagentur, ihr folgen Regionaldirektionen, die die Verantwortung für den Erfolg der regionalen Arbeitsmarktpolitik tragen und mit den Landesregierungen die Leistungen der Arbeitsförderung mit deren Arbeitsmarkt-, Struktur- und Wirtschaftspolitik abstimmen ( $\$ 367$ Abs. 3 SGB III). In einigen Fällen erstreckt sich eine Regionaldirektion über zwei Bundesländer, in einem über drei. Auf der lokalen Verwaltungsebene

57 Vor dem 01.01.2004 war die Bundesanstalt für Arbeit Trägerin der Arbeitsförderung.

58 Er kann etwa vom Vorstand Prüfungen durch die Interne Revision verlangen. 
befinden sich die Agenturen für Arbeit. Die Organisation der Bundesagentur für Arbeit umfasst die Zentrale in Nürnberg, zehn Regionaldirektionen, 156 Agenturen für Arbeit und circa 600 Dependancen, 304 Jobcenter (gemeinsame Einrichtungen), die von den Agenturen für Arbeit vor Ort mit kreisfreien Städten beziehungsweise Landkreisen gebildet worden sind (BA 2013f).

Als klassische Behörde entsprach ihr Aufbau und ihre Funktionsweise vom Arbeitsförderungsgesetz (AFG) bis zum SGB III (im SGB II hingegen ein wenig schwächer) lange den Prinzipien des traditionellen Bürokratiemodells mit hierarchischer, amtsförmiger Linienorganisation sowie Recht und Legalität als dominantem Steuerungsmodus - einer konditionalen Regelsteuerung (Schütz 2012). Mit den Arbeitsmarktreformen kam verstärkt eine quantitative Zielsteuerung zum Einsatz, unter anderem über die bundesweite und die lokale Integrationsquote. So wurden Elemente der Konditionalsteuerung und Elemente der wirkungsorientierten (Ziel-)Steuerung in eine neue Form der Regelsteuerung überführt (Schütz 2012: 245).

\subsubsection{Lokale SGB-II-Träger}

Lokale SGB-II-Träger sind mit der lokalen Umsetzung der Grundsicherung für Arbeitsuchende im SGB-II-Bereich betraut. Ihre Aufgabe ist es, Leistungen nach dem SGB II zu gewähren und mittels des Prinzips »Fördern und Fordern « ${ }^{59}$ Menschen in Erwerbstätigkeit zu integrieren. Die SGB-II-Träger operieren in zwei verschiedenen Organisationsformen: 1.) der ehemaligen Arbeitsgemeinschaft (ARGE) und 2.) dem zugelassenen kommunalen Träger (zkT, auch Optionskommune genannt). ARGEn waren Arbeitsgemeinschaften aus lokalen Agenturen für Arbeit (AA) und der Kommune, die mit der Umsetzung der Aufgaben des Sozialgesetzbuches II betraut waren. Da ein Bundesverfassungsgerichtsurteil 2007 die gemeinsame Aufgabenwahrnehmung von Langzeitarbeitslosen durch Kommunen und die Bundesagentur für Arbeit für verfassungswidrig erklärte (BVerfG 2007), wurde die Trägerschaft im Jahr 2010 neu geregelt. Die gemeinsamen Einrichtungen (von Kommune und Agentur für Arbeit) nach § 44b SGB II und die zugelassenen kommunalen Träger (Optionskommunen) nach § 6a SGB II führen nun einheitlich die Bezeichnung »Jobcenter « ${ }^{60}$ (nach § 6d SGB II). Dort werden weiterhin die Leistungen von einem Standort aus vergeben, die Bundesagentur für Arbeit ist jedoch zuständig für Bundesmittel, wie

59 Vergleiche Kapitel 4.6.2 »Fördern und Fordern« als Motor der Aktivierung.

60 Der Begriff Jobcenter geht auf JobCenter aus dem Abschlussbericht der Kommission »Moderne Dienstleistungen am Arbeitsmarkt« zurück (Hartz-Kommission 2002). 
Leistungen der Grundsicherung und arbeitsmarktpolitische Instrumente. Kommunen übernehmen gemäß $§ 22$ SGB II Kosten der Unterkunft und Heizung $(\mathrm{KdU})$, einmalige Sonderleistungen und soziale Dienstleistungen. Die Jobcenter wurden dabei in ihrem jeweiligen Modell der Trägerschaft (gemeinsame Einrichtung aus Kommune und Agentur für Arbeit respektive kommunale Träger) entfristet. Die frühere dritte Option der Organisation als SGB-II-Träger, Einrichtungen der getrennten Aufgabenwahrnehmung (gAW), wurde 2012 abgeschafft, war jedoch nicht Bestandteil der dieser Arbeit zugrunde liegenden Empirie. Da die heutigen Jobcenter zum Erhebungszeitpunkt teilweise noch als Arbeitsgemeinschaft galten beziehungsweise von den GesprächspartnerInnen so bezeichnet wurden, sind in dieser Untersuchung beide Begriffe zu finden.

Daher gewähren Jobcenter passive Leistungen zum Lebensunterhalt nach dem SGB II und aktive Eingliederungsleistungen, wie Weiterbildungen, Eingliederungszuschüsse oder Beschäftigungsmaßnahmen (teils nach dem SGB III). Die Betreuung von Langzeitarbeitslosen, die die Zielgruppe der hier als Maßnahmen Sozialer Aktivierung bezeichneten Maßnahmen sind, wird durch das Fallmanagement und die Arbeitsvermittlung vorgenommen. Die Vermittlungspraxis wurde im SGB-II-Bereich für arbeitsmarktferne Personen vielfach in einem Fallmanagement organisiert und dabei lokal in unterschiedlichen Organisationvarianten in den Vermittlungsbereich integriert oder aber von diesem entkoppelt (BMAS 2008), wobei die zugelassenen kommunalen Träger eigene Vermittlungsvarianten durchführen. Zwar gehen die Vermittlungskräfte mit unterschiedlichen Strategien an die Betreuung von Langzeitarbeitslosen und die Vergabe von Leistungen heran (Boockmann/Koch/Rosemann/Stops/Verbeek 2010), doch wird hier häufig thematisiert, dass niederschwellige Maßnahmen für eine sehr weit vom Arbeitsmarkt entfernte Klientel benötigt werden.

Für SGB-II-Träger waren langwierige (und auch gerichtlich ausgetragene) Einführungs- und Fortentwicklungsprozesse hinsichtlich einer kontinuierlichen Organisationsentwicklung belastend, da aufgrund der prozessbedingten Planungsunsicherheiten kaum stabile, entfristete Arbeitsverträge mit Fachkräften abgeschlossen werden konnten (Schütz et al. 2011: 323). Dies führte zu Ineffizienzen in der Personalsituation, da fachkundiges Personal nicht weiter beschäftigt werden konnte. So wurde festgestellt, dass Fachkräfte vereinzelt unzureichend über gesetzliche Bestimmungen oder Maßnahmen informiert waren (Schütz et al. 2011: 262). Zwar wird die Arbeit der SGB-II-Träger von den Alg II-Leistungsempfangenden als Dienstleistung wahrgenommen, jedoch wird das eigentliche Ziel der Arbeitsmarktintegration nicht immer durch das Wirken der Fachkräfte unterstützt (Tisch 2010). 


\subsubsection{Maßnahmenträger}

Die SGB-II-Träger und auch die Arbeitsagenturen verfügen über ein Budget aus dem Eingliederungstitel (Bundesmittel für die Leistungen zur Eingliederung in Arbeit) anhand dessen sie Arbeitsmarktdienstleistungen von Maßnahmenträgern einkaufen.

Maßnahmenträger kommen in unterschiedlichen Organisationsformen und inhaltlichen Ausrichtungen vor: Im Sample existieren etwa ein christlicher Jugendträger, ein sozialbetrieblicher, familienorientierter und frauenbezogener Maßnahmenträger sowie Bildungsträger. So haben die Maßnahmenträger zum Teil bereits eine spezifische Zielklientel oder spezifische Werte, die sie im Rahmen der Beschäftigungsförderung vermitteln möchten. Die Maßnahmenträger im Sample treten privatwirtschaftlich oder in Form eingetragener Vereine auf und sind teils regional und teils überregional vertreten. Mit Bode (Bode 2011: 323f.) differenziere ich das Akteursfeld der Maßnahmenträger in klassische Sozialorganisationen, Träger direkter Beschäftigungsförderungsmaßnahmen und gewerbliche Anbieter: Maßnahmenträger selbst können klassische Sozialorganisationen sein, die in Trägerschaft der Kommune sind oder der freien Wohlfahrtspflege angehören. Sie erhalten meist Fallpauschalen und erbringen Schulungs-, Trainings- und Weiterbildungsdienstleistungen, wie beispielsweise auch Schuldnerberatung und psychosoziale Betreuung. Arbeitsmarktbezogene soziale Dienstleistungen werden von Trägern direkter Beschäftigungsförderungsmaßnahmen (Bode 2005) erbracht, wie gemeinnützige Einrichtungen (Vereine oder gGmbHs) und kommunale Eigenbetriebe. Des Weiteren agieren gewerbliche Anbieter wie Zeitarbeitsfirmen, spezialisierte Agenturen sowie gewerbliche Bildungsträger in diesem Bereich.

Maßnahmenträger, die beispielsweise Maßnahmen der beruflichen Weiterbildung und Maßnahmen zur Aktivierung und beruflichen Eingliederung ( $\$ 45$ SGB III) anbieten möchten, ${ }^{61}$ müssen eine Trägerzulassung besitzen. Künftig sollen alle Maßnahmenträger sowie Maßnahmen ${ }^{62}$ einem Zulassungsprozess unterzogen werden (BA 2013a). Derzeit bewerben sich Maßnahmenträger im

61 Wenn sie mit einem Aktivierungs- und Vermittlungsgutschein gefördert werden. Dies gilt nicht für Vergabemaßnahmen und die Unterstützung der Vermittlung in versicherungspflichtige Beschäftigung nach $\S 45$ Abs. 1 Satz 1 Nr. 3 SGB (BA 2013a).

62 Auch von den Maßnahmenträgern angebotene Maßnahmen der beruflichen Weiterbildung und künftig auch Maßnahmen zur Aktivierung und beruflichen Eingliederung nach § 45 SGB III, die im Rahmen des Aktivierungs- und Vermittlungsgutscheins gefördert werden, benötigen eine Zulassung. 
Rahmen von Ausschreibungen mit ihren Maßnahmenangeboten in einem rechtlich-geregelten Vergabeverfahren. ${ }^{63}$ Für die unmittelbar mit den bewilligten Maßnahmen entstehenden Aufwendungen werden den Maßnahmenträgern Kosten erstattet. Meist wird neben einem Grundbetrag eine Vermittlungsprämie ausgezahlt. Die Finanzierung sowie die Maßnahmenvergabe der jeweiligen Maßnahmen differiert jedoch nach Mittelgeber (SGB-II-Träger, Bildungsgutschein, ESF-Fonds, Perspektive 50plus etc.).

Mit der Hartz-Gesetzgebung verstehen sich auch zahlreiche Maßnahmenträger als arbeitsmarktpolitischer Dienstleister und richten Beschäftigungsförderungsmaßnahmen auf eine schnellstmögliche Vermittlung in Erwerbstätigkeit aus, oftmals zu Ungunsten zivilgesellschaftlicher Verwurzelung (Bode 2011: 328). Doch trotz eingeschränkter Handlungsspielräume sind zahlreiche Maßnahmenträger in vielfältiger Weise wirtschaftlich tätig sowie mit dem lokalpolitischen wie zivilgesellschaftlichen Umfeld verbunden, um in Projekten gerade auch Armut zu bekämpfen und das lokale soziale und kulturelle Dienstleistungsangebot sowie die regionale infrastrukturelle Entwicklung $\mathrm{zu}$ fördern (Bode 2011: 328). ${ }^{64}$

Insgesamt ersetzt verstärkt eine quantitative Zielsteuerung die frühere konditionale Regelsteuerung der Bundesagentur für Arbeit und der ihr untergebenen Institutionen - den Regionaldirektionen, Agenturen für Arbeit und SGB-II-Trägern. Die lokalen SGB-II-Träger kaufen zum Zweck der Beschäftigungsförderung über den Eingliederungstitel unter anderem Maßnahmen Sozialer Aktivierung von einem breiten Angebotsfeld diverser Maßnahmenträger ein.

63 Vergleiche Kapitel 5.2 Rechtlicher und finanzieller Rahmen für die Schaffung sozial aktivierender Maßnahmen.

64 Für Zielsetzungen, die von Maßnahmenträgern mit Maßnahmen Sozialer Aktivierung verbunden werden, siehe Kapitel 5.5.1 Institutionenspezifische Funktionen. 



\section{Maßnahmen Sozialer Aktivierung: Ziele, Genese und Funktionen}

Nachdem der Kontext der Formation des Aktivierungsdiskurses sowie das Akteursfeld der Arbeitsverwaltung und Maßnahmenträger dargestellt wurden, sollen nun die auf eine sehr arbeitsmarktferne Klientel zielenden Maßnahmen Sozialer Aktivierung beschrieben werden. Zu diesem Zweck werden die neuartigen Instrumente der Beschäftigungsförderung im Kapitel 5.1 zunächst definiert und in Fallbeispielen charakteristischer Maßnahmeninhalte dargestellt. Im Kapitel 5.2 gilt es ihre rechtlichen und finanziellen Rahmenbedingungen sowie Entstehungswege aufzuzeigen, die sich von typischen Instrumenten der Beschäftigungsförderung unterscheiden. Dieser institutionelle und normative Rahmen verweist auf eine Besonderheit in der Maßnahmengenese. In Kapitel 5.3 wird rekonstruiert, inwiefern sich trotz dieses Kontextes eine institutionalisierte Rechtsform für die Maßnahmen Sozialer Aktivierung etabliert hat. Gleichzeitig bestehen jedoch Spannungsfelder zwischen den normativen Anforderungen des Aktivierungsdiskurses und der Praxis der Arbeitsverwaltung, die auch durch die Installierung von Maßnahmen Sozialer Aktivierung zu Tage treten und Gegenstand der Analyse in Kapitel 5.4 sind. Im Kapitel 5.5 werden die Funktionen des neuartigen ganzheitlichen Beschäftigungsförderungsinstruments rekonstruiert.

\subsection{Massnahmen Sozialer Aktivierung: EINE DEFINIERENDE DARSTELLUNG}

Wie so häufig, verdanken sich die im Zentrum stehenden Forschungsfragen auch bei dieser Studie einer glücklichen Koinzidenz virulenter gesellschaftlicher Anliegen und eines Zufallsfundes in Gestalt positiver Äußerungen über einen Kampfsport-Kurs von >bekennenden Arbeitslosen` in einem Online-Chat. Mit 
diesem Kurs als Teil einer öffentlich finanzierten Maßnahme, tritt ein Aspekt der Beschäftigungsförderung zutage, der allein aus den zentral-administrativen Daten der Bundesagentur für Arbeit nicht ersichtlich wäre. Begeistert tauschten sich im besagten Chat Langzeitarbeitslose über die individuellen Fortschritte im Training aus und besprachen, wie (selbst-)gesteckte Ziele erreicht wurden, so dass sie nun >viel aufrechter durchs Leben` gehen könnten. An dieser Stelle lässt sich aus der Beobachterperspektive die Überlegung anschließen, ob dieses positive Grundgefühl wiederum ein Ansporn für die Bewerbungsbemühungen auf dem Arbeitsmarkt liefern könnte. Die Rezeption dieser Korrespondenz führte die Autorin zu der Annahme, dass neben den üblichen Maßnahmen des Bewerbungstrainings, der schulischen und beruflichen Qualifizierung etc. eine Maßnahmenform existieren könnte, die zunächst nicht arbeitsmarktqualifizierend erscheint und doch von der Arbeitsverwaltung eingesetzt wird, als neuartiges Instrument der Beschäftigungsförderung, um Personen an den Arbeitsmarkt heranzuführen. Im Laufe der Akquise nach solchen nicht-arbeitsähnlichen, im Feld häufig »ganzheitlich« oder »niederschwellig« genannten Maßnahmen, sollte sich herausstellen, dass eine höchst facettenreiche Maßnahmenpraxis an entsprechenden niederschwelligen Angeboten existiert. Dieser Maßnahmentyp soll unter der im Zusammenhang dieser Abhandlung gewonnenen Bezeichnung Maßnahmen Sozialer Aktivierung dargestellt werden.

Maßnahmen Sozialer Aktivierung sind eine hier konzeptualisierte Kategorie. Sie umfassen Maßnahmen der Beschäftigungsförderung, denen im Feld persönlichkeitsstabilisierende und sozial integrierende Effekte zugeschrieben werden und die nur in einer langfristigen Perspektive als arbeitsmarktbezogene Förderung angesehen werden können. Sie liegen damit jenseits der expressis verbis im SGB II und SGB III genannten Ziele von Arbeitsmarktintegration und Verbesserung der Beschäftigungsförderung. Dieser Maßnahmentyp setzt demnach weniger auf die unmittelbare Vermittlung in den ersten Arbeitsmarkt, sondern bietet vielmehr sehr niederschwellige Angebote zur Unterstützung der Alltagskompetenz (wie die Pflege des Äußeren, Mobilität im nahen Wohnraum, Zeitstruktur) und der Selbstwirksamkeit (wie das Stimmtraining, Auftreten, eigene Hürden überwinden). Formell werden solche Maßnahmen als Vorstufe oder flankierendes Modul zur Arbeitsmarktintegration angesehen. Der verwendete Begriff orientiert sich an einem 1996-2003 durchgeführten niederländischen Arbeitsmarktprogramm (»Social Activation«), in dem Langzeitarbeitslosen mit wenig Aussicht auf eine Erwerbstätigkeit, Alternativen gesellschaftlicher Partizipation ermöglicht werden sollten: soziale Kontakte, Tagesstruktur und Förderung des Selbstbewusstseins. Befristet von der Jobsuche freigestellt, erhielten die Teil- 
nehmenden für ehrenamtliches Engagement ein Entgelt (Europäische Kommission 2007).

Der Maßnahmentyp Sozialer Aktivierung ist insofern von anderen Maßnahmen abzugrenzen, als er unabhängig von der rechtlichen Realisierungsform seinen Fokus auf die Aktivitäten der Maßnahmenteilnehmenden und das Verständnis der professionellen AkteurInnen und der beteiligten Institutionen von diesen $\mathrm{Ma}$ ßnahmen richtet. Die Förderstrukturen der Bundesagentur für Arbeit sowie das Controlling ergeben sich in der Regel aus der Finanzierungslogik der Arbeitsmarktdienstleistungen und spiegeln sich auch in den BA-Statistiken zur Beschäftigungsförderung (zum Beispiel BA 2013e) sowie in den Veröffentlichungen und dem Forschungsprogramm des Instituts für Arbeitsmarkt- und Berufsforschung der Bundesagentur für Arbeit (zum Beispiel IAB 2013) wieder. Hier werden Maßnahmen häufig im Rahmen ihrer Gesetzesform untersucht wie beispielsweise Arbeitsgelegenheiten nach § 16d SGB II oder der Gründungszuschuss nach $\S 93$ SGB II. Die Kategorie der Maßnahmen Sozialer Aktivierung erstreckt sich hingegen über mehrere Gesetzes- beziehungsweise finanzielle Realisierungsformen. ${ }^{1}$ Maßnahmen Sozialer Aktivierung sind so durch Maßnahmeninhalte charakterisiert, wie die Tätigkeiten der Teilnehmenden in der Maßnahme sowie die damit verbundene Zielsetzung durch SGB-II- und Maßnahmenträgerbeschäftigte. Gemeinsam ist den Maßnahmeninhalten, dass diese sich mit (unmittelbar) arbeitsmarktfernen Aspekten beschäftigen, die zunächst aus Sicht der Arbeitsverwaltung ${ }^{2}$ auf soziale Aktivierung zielen und via mitteloder langfristiger Perspektive eine Arbeitsmarktintegration bewirken sollen. Die Tätigkeiten umfassen ein Spektrum arbeitsmarktferner Inhalte (wie Kurse zur Rückengymnastik, Ernährung, Stressbewältigung oder Haushaltsführung), kulturelle Maßnahmen (wie Tanzkurse und Theatergruppen), aber auch Leistungen Dritter (wie Schuldner- und Suchtberatung, sozialpädagogische Betreuung) sowie die Förderung von basalen Alltagskompetenzen. Im Folgenden werden fünf Fallbeispiele $^{3}$ von Maßnahmen Sozialer Aktivierung en détail dargestellt.

1 Vergleiche Kapitel 5.2.3 Rechtliche Realisierungsform und lokale Umsetzungen der Maßnahmen Sozialer Aktivierung.

2 D.h. Jobcenter, ehemalige Arbeitsgemeinschaften und Maßnahmenträger.

3 Die Fallbeispiele basieren auf Fallstudien eines IAB-internen Berichtes der Autorin für das BMAS (Freier 2012). 


\subsubsection{Fallbeispiel einer sozialarbeiterischen U25-Maßnahme in sozialem Brennpunkt}

Die Maßnahme zur Aktivierung besonders benachteiligter Jugendlicher (nach § 16f. Abs. 1 SGB II, Freie Förderung) hat eine zwölfmonatige Laufzeit und bietet in zwei Maßnahmenphasen maximal 100 unter 25Jährigen eine Teilnahme: 1.) die geschlossene Form mit 60 Plätzen und täglicher Anwesenheitspflicht sowie 2.) die flexiblere Form mit 40 Plätzen bei Abwesenheitszeiten der Jugendlichen entsprechend ihrem Bedarf. Daneben gibt es einen offenen Begegnungsbereich, in dem Jugendliche aus dem Stadtteil sowie frühere, aktuelle oder potentielle Maßnahmenteilnehmende sich sozialpädagogisch begleitet austauschen können.

Die Einrichtung, die nicht nur darauf ausgerichtet ist, ein familiäres Umfeld und Zeit für Begegnung zu bieten, geht über Aufgaben üblicher Maßnahmenträger insofern hinaus, als hier eine 24 Stunden nutzbare Anlauf- und Beratungsstelle mit Notunterkunft für wohnungslose Jugendliche angeboten und bedarfsweise auch Kontakt mit den Eltern herstellt.

Wenn Jugendliche, die an der anwesenheitspflichtigen Maßnahmenvariante teilnehmen, einer Einladung zum Maßnahmenträger nicht nachkommen, führen die Maßnahmenträgerbeschäftigten aufsuchende Dienste durch. Im Selbstverständnis der Beschäftigten ist damit eine Form persönlicher Interessensbekundung und Wertschätzung gegenüber den Jugendlichen intendiert (Interview03Regio04). Eine Geste der Wertschätzung sei zum Beispiel, dass die Teilnehmenden für die Fahrzeuge des Maßnahmenträgers ohne weitere Kontrolle den Zündschlüssel zur Übernahme kleinerer Erledigungsfahrten erhalten. Entgegengebrachter Respekt sowie die zugesprochene Verantwortung führten dazu, dass die Autos bisher nie zweckentfremdet worden seien, sich die Vertrauensbasis festige und das Selbstbewusstsein der Teilnehmenden gestärkt werde.

Nach einem Erstgespräch werden die Jugendlichen im offenen Treff eingeführt, der eher an eine soziale Jugendeinrichtung erinnert. Maßnahmeninhalte sind ein sozialpädagogisch begleitetes, tägliches Frühstück und Mittagessen mit allen DozentInnen sowie der Trägerleitung in familiär-anmutender Atmosphäre. Kreative Angebote (Zirkusgruppe und Rap-Studio) schließen sich nachmittags an die Bildungsangebote an. In Holz-, Metall- und Malerwerkstätten können Jugendliche vormittags für den Eigenbedarf produzieren und neue Arbeitsfelder erproben. Politische Bildung ist ebenfalls Gegenstand der Maßnahme. Darüber hinaus werden Ausflüge sowie Arbeitseinsätze im Stadtgebiet und jenseits der Landes- 
grenzen unternommen. Die Ausflüge werden eingesetzt um, die häufig starke Fixierung der Jugendlichen auf ihren unmittelbaren Wohnraum zu lösen - die psychisch-räumlichen Grenzen zu überwinden. Ferner können Schulabschlüsse bei einer Trägerniederlassung in einem anderen Bundesland nachgeholt werden.

Die Maßnahme richtet sich an erwerbslose, sehr beeinträchtigte und sozial benachteiligte Menschen unter 25 Jahren, die Angebote der Berufsorientierung, der Ausbildung oder Beschäftigung aufgrund persönlicher Gründe nicht in Anspruch nehmen können. Charakteristisch seien wohnungslose, suchterkrankte Jugendliche ohne Schulabschluss mit traumatischen Erfahrungen in der Kindheit sowie Problemen im Elternhaus.

Ziel der Maßnahme sei es, diese Klientel zu erreichen, die sich üblicherweise dem Jobcenter zu entziehen suche. Ferner gelte es, Arbeitstugenden und Sozialkompetenzen zu kultivieren sowie das Selbstbewusstsein der Teilnehmenden zu stärken und Zukunftsoptionen zu eruieren. »Da fängt soziale Integration an, denke ich. Also nicht nur so lang zu laufen und sich selbst als Hartz IVer oder als Nichtschulabgänger oder als Looser zu denken. Sondern mal wieder ganz mutig geradeaus zu gucken.« (Maßnahmenträgerleitung, Interview03Regio04)

In äquivalenten Maßnahmen wird betont Leistungen zu gewähren, während die Unterstützungsnehmenden unabhängig von den individuellen Problemlösungsfähigkeiten weiterhin einen Status der Autonomie innehaben. Das in der Sozialen Arbeit vielfach rezipierte Konzept des Empowerments folgt dieser Logik ebenfalls. Mit den Worten Julian Rappaports:

»Unter >empowerment< verstehe ich, daß es unser Ziel sein sollte, für Menschen die Möglichkeiten zu erweitern, ihr Leben zu bestimmen. [...] Mit dem Konzept >empowerment< können wir nicht länger Menschen einfach als `Kinder in Notく oder als `Bürger mit Rechten sehen, sondern vielmehr als vollwertige Wesen, die sowohl Rechte als auch Bedürfnisse haben.« (Rappaport 1985: 289)

Das Empowerment wird insbesondere darüber angestrebt, der Angst vor Neuem entgegenzutreten (etwa über Ausflüge, neue Fähigkeiten erkennen). Dies wirke sich positiv auf das Selbstwertgefühl aus, und sei zugleich Voraussetzung für eine aussichtsreiche Stellensuche. In einer aus 50plus-Mitteln finanzierten Maß- 
nahme mit »Empowerment-Ansatz« ${ }^{4}$ (Maßnahmenträgerleiterin, Interview01Regio02) werden Gruppenkonzepte gewählt, um Kompetenzen zu entwickeln, die auch den Kontakt mit dem ersten Arbeitsmarkt betreffen können, wie telefonisches Organisieren von Bewerbungsterminen für andere Gruppenmitglieder und gegenseitiges Einüben von Bewerbungsgesprächen (Interview01Regio02). Auch Fähigkeitsstände werden eruiert, etwa Analphabetismus, Sprachbarrieren, mangelnde Problemlösungskompetenzen. Derartige Defizite können eine zielgerichtete Arbeitsvermittlung behindern und werden bisweilen in der üblichen Interaktion mit dem SGB-II-Träger nicht erkannt. Die zum Teil schambesetzten und sozial stigmatisierenden Themen sollen im intensiven Austausch zwischen Teilnehmenden und Maßnahmenträgerbeschäftigten zu Tage treten.

\subsubsection{Fallbeispiel einer sozial aktivierenden Maßnahme mit Sportelementen für Jugendliche}

Erstmals nutzte ein überregional agierender Bildungsträger Elemente der Sozialen Aktivierung, als er Sportelemente in zweijährige ESF-finanzierte Maßnahmen integrierte. Seitdem besuchen 25 Personen unter 25 Jahren mit Migrationshintergrund dreimal wöchentlich neunzigminütige Kung-Fu-Einheiten als Pflichtveranstaltung neben berufsqualifizierenden Bildungsangeboten (wie EDV- und kaufmännischen Kursen).

Die Kung-Fu-Kurse sind auf ein Austesten der persönlichen Leistungsgrenzen angelegt. Sie sollen individuell sportlich leistbar und zugleich herausfordernd sein. Die Trainingseinheiten übernimmt ein KungFu-Meister, der über Zusatzqualifikationen für Gesundheitsgymnastik verfügt. Der Kursleiter arbeitet auch für andere Träger, die in Kooperationsprojekten das Maßnahmenkonzept kennen lernten. ${ }^{5}$

4 Nach einer Kennenlernphase werden in Kleingruppen notwendige Schritte zur Stellensuche aufgeteilt (Bewerbung schreiben, Kaltakquise, Internetrecherche etc.) und für alle Teilnehmenden ausgeführt, aber auch Qualifizierungseinheiten in Workshops von Teilnehmenden für Teilnehmende durchgeführt (Interview01Regio02).

5 Auch bei der Ausdehnung des Sportangebotes (Tanzkurse für über 50-Jährige) arbeitet der Maßnahmenträger mit einem externen Anbieter zusammen, einer Tanzschule. Die erleichterte Einbeziehung von Sporteinheiten könnte mit dem »Kampagnenjahr« 2009 des Bundesprogramms »Perspektive 50plus« zusammenhängen, in dem unter anderem ein Schwerpunkt auf der Gesundheitsförderung mit Partnern aus dem Gesundheitssektor lag (BMAS 2010). 
In den Trainingseinheiten erlernte soziale und personale Kompetenzen werden als wesentliche Voraussetzung für den Eintritt in das Erwerbsleben betrachtet: »Der Mensch muss erst bereit sein, sich auf etwas Neues einzulassen und das auch wollen. Und das alles ihm bewusst $\mathrm{zu}$ machen, ey Du kannst, Du kannst viel, schau hin, was Du alles kannst.« (Maßnahmenträgerleiterin, Interview01Regio02) ${ }^{6}$

Darüber hinaus sei für spezifische Personengruppen (insbesondere Jugendliche) die Herstellung einer Vertrauens- und der grundlegenden Kommunikationsbasis erst möglich, wenn sie auf einem Gebiet angesprochen werden können, das sie berührt, das für sie mit Spaß verbunden sei.

Die physische Konstitution der Personen im SGB-II-Bezug, die an Maßnahmen Sozialer Aktivierung teilnehmen, kann durch verschiedenste Erkrankungen beschränkt sein: beispielsweise Beeinträchtigungen im Muskel-Skelett-System, Ernährungs- und Stoffwechselstörungen oder Suchtkrankheiten. Ähnlichen Maßnahmen des gesundheitsbezogenen Verhaltens wird seitens der SGB-II- und Maßnahmenträger die Funktion zugeschrieben, diesen Beeinträchtigungen entgegenzuwirken, respektive den Teilnehmenden zu ermöglichen, die eigenen Grenzen und Stärken zu erkennen und für mögliche Beschäftigungsformen trotz der gesundheitlichen Einschränkung zu sensibilisieren. Ferner bewirke die Aktivierung über Sportangebote eine Offenheit für die Stellensuche und die Fähigkeit, eigene Fertigkeiten realistisch einzuschätzen. Diese entstünden erst dann, wenn ein bestimmtes soziales Klima und eine bestimmte Motivation vorhanden seien. Die Freisetzung beziehungsweise Entstehung eines solchen eigenen Antriebes wird dem Kanalisieren der Energie im Training, dem Spüren körperlicher Grenzen und den individuellen Erfolgen durch die Überwindung dieser eigenen Limits zugeschrieben, genauso das Erlernen von Disziplin und die damit verbundenen positiven Effekte auf das soziale Miteinander.

6 Hier und im Folgenden wurden die Zitate aus den Interviews aus Gründen der besseren Lesbarkeit sprachlich geglättet, indem Verzögerungslaute wie »ähm«, »mhm« entfernt wurden. 


\subsubsection{Fallbeispiel einer Maßnahme zum Heranführen an geordnete Familienstrukturen und -prozesse}

Im Rahmen ESF-geförderter Kleinprojekte mit einer Fördersumme von bis zu 10.000 Euro bietet ein an eine Frauenvereinigung angegliederter Maßnahmenträger Veranstaltungen hauptsächlich für erwerbslose Frauen an. Die zwei bis drei wöchentlichen Treffen respektive Vortragsveranstaltungen sind stark auf den Gruppenaustausch und eine individuelle Gestaltung der Maßnahme ausgerichtet. Übergeordnete Themenbereiche werden im Voraus festgelegt, doch die konkrete Ausgestaltung übernimmt die Teilnehmerinnen-Gruppe meist in Eigenregie. Themen sind dabei Kindererziehung, Haushaltsführung, Gesundheitsorientierung und Fragen der alltäglichen Lebensführung.

Im Bereich des Heranführens an eine höhere Erziehungskompetenz werden Vorträge zur spielerischen Beschäftigung mit Vorschulkindern organisiert, Basteltätigkeiten und kreative Betätigungen für Mutter und Kind mit den Müttern eingeübt (Quillingtechnik, Laternen basteln etc.). Auch Optionen für die Freizeitgestaltung der Kinder mit einfachen respektive kostengünstigen Mitteln zu Hause und spielerisches Lernen werden aufgezeigt. Ein Büchereibesuch ist inbegriffen und soll neben der Vermittlung, wo Sachbücher und arbeitsmarktbezogene Literatur zu finden sind, ebenso an Kinderbücher heranführen. Neben Erziehungsthemen werden auch Eheverhältnisse diskutiert sowie die Frage, inwiefern sich Frauen von ihren Partnern emanzipieren können.

Im Bereich der Haushaltsführung wird regelmäßig ein Besuch der lokalen Tafel und des Sozialkaufhauses unternommen, um über alternative Einkaufsoptionen aufzuklären. Eine Gruppe, die den Wunsch hatte, das Körpergewicht zu reduzieren, wurde über gesunde Ernährung durch eine Ernährungsberaterin einer Krankenkasse und planendes, preisbewusstes Einkaufen informiert.

Regelmäßig finden in den sonst für den Unterricht oder Veranstaltungen genutzten Räumen soziale Zusammenkünfte statt, bei denen teils auch eigenständig gehäkelt, gestrickt, gezeichnet oder gebastelt wird.

Effekte, die mit diesem Projekt vom Maßnahmenträger bezweckt werden, sind eine gesteigerte Kommunikationskompetenz, die Stärkung des Selbstbewusstseins und Persönlichkeitsförderung durch das Herausfinden eigener vielfach ungeahnter lebenspraktischer Kompetenzen sowie das Feedback in der Gruppeninteraktion. Insbesondere die sozialen Kompetenzen in den Interaktionen beim Frauenfrühstück oder in der ge- 
selligen Kaffeerunde werden als Mittel verstanden, um eher sozial isolierte Personen einzubinden und diese auch längerfristig nach dem Projekt an die Institution zu binden. Darüber hinaus möchte der Maßnahmenträger Qualifikationen, wie gute Kindererziehung, gesunde Ernährung und ordentliche Haushaltsführung vermitteln.

In solchen Maßnahmen, die soziale und familiale Verhältnisse sichten, wird bei der Suche von Kinderbetreuungseinrichtungen sowie im alltäglichen Familienleben unterstützt, etwa Erziehungsprobleme besprochen, Zeitstrukturen für das Familienleben aufgezeigt oder Reparaturen respektive Herstellen von Kinderkleidung eingeübt. ${ }^{7}$ Traumatisierungen wie (sexuelle) Gewalterfahrungen als TäterIn respektive Opfer und Suchtprobleme im Elternhaus beziehungsweise im sozialen Umfeld sollen beim Maßnahmenträger zur Sprache kommen und nach ihrer Identifizierung in Kooperation mit dem SGB-II-Träger bearbeitet werden. Der Maßnahmenträger verweist gegebenenfalls an die existierenden sozialen und therapeutischen Einrichtungen und unterstützt bei Einstieg und Fortsetzung dieser Hilfsangebote. ${ }^{8}$

\subsubsection{Fallbeispiel einer Maßnahme zur Bereitstellung psychisch- physischer Unterstützungsangebote sowie gesundheitsorientierter Beschäftigungsvermittlung}

Im Rahmen einer Arbeitsgelegenheit (nach $§ 16 d$ SGB II) wird eine individuelle zielorientierte Problemberatung in einer Maßnahme kombiniert mit Tätigkeiten, die am psychosozialen und physischen Gesundheitszustand orientiert sind. Die Beratungsstruktur realisieren ein Sozialmediziner und eine Psychologin. Der Sozialmediziner wertet hauptsächlich ärztliche Gutachten der Agentur für Arbeit aus und gibt Empfehlungen für am gesundheitlichen Zustand orientierte individuelle Arbeitsmöglichkeiten oder zur Aufsuche weiterer (Fach-)Ärzte. Die Psychologin unterstützt bei der Aufnahme einer Therapie oder anderer Angebote Dritter und

7 Derartige Ansätze finden sich beispielsweise bei dem frauenbezogenen Maßnahmenträger in Regio11, dem familienorientierten Maßnahmenträger (Interview06Regio06) und einem regionalen Maßnahmenträger (Interview03Regio10).

8 Die Bedeutung der Identifizierung von Problemlagen durch die Maßnahmenteilnahme in Maßnahmenträgern wird insbesondere im U25-Bereich in Regio12 herausgestellt (Teamleiterin U25 Interview01Regio12). 
führt Eignungstests durch. Im Fallmanagement wird eine am Gesundheitszustand der Person orientierte Maßnahme oder Beschäftigungsmöglichkeit ermittelt.

Sozial aktivierende Elemente sind Sportangebote sowie eine Theaterwerkstatt. Die durch die beteiligten Krankenkassen finanzierten Module der Ernährungsberatung, Rücken- und Präventionsgymnastik und Yoga sind primär gesundheitlich ausgerichtet. Daneben werden sie eingesetzt, um den Gruppenzusammenhalt und das individuelle Selbstbewusstsein zu stärken. Das einwöchige Kommunikationstraining einer Theaterdramaturgin orientiert sich mittelbar am Arbeitsmarkt und ist zur Verbesserung der Selbstwahrnehmung, des Entdeckens eigener Stärken und der Entwicklung kommunikativer Fähigkeiten eingeführt worden.

Die Klientel dieser Maßnahme hat aus Sicht der SGB-II-Träger- und Maßnahmenträgerbeschäftigten, physische und/oder psychische Einschränkungen, die häufig bereits vor der Arbeitslosigkeit existierten und sich während der Erwerbslosigkeit intensivierten. Häufig seien diese Teilnehmenden suchterkrankt. Das häufigste Thema in der Beratung ist der Umgang mit der Arbeitslosigkeit. Für die Psychologin ist deshalb klar: »Es tut ihm gut, einfach morgens wieder eine Arbeitsstruktur zu haben. Raus zu gehen, körperliche Arbeit zu haben. Er hatte Schlafprobleme [...] und seit er in diesem Projekt drin ist, sagt er: ich schlafe wunderbar.« (Interview01Regio05)

Aus Sicht der Psychologin sei gerade bei weiblichen Erwerbslosen die Steigerung der Selbstwirksamkeit durch die Vergegenwärtigung eigener Fähigkeiten bedeutend. Bei Älteren sei das Leben- und ArbeitenLernen mit körperlichen Einschränkungen oder chronischen Krankheiten, nebst dem Aufzeigen alternativer Fähigkeiten, von Bedeutung. Weitere Ziele der Maßnahme seien, die für dieselbe Person zuständigen Beschäftigten der Krankenkassen und SGB-II-Träger zu vernetzen, die Erwerbsfähigkeit zu testen sowie gegebenenfalls an Rentenversicherungsträger oder den Reha-Bereich zu vermitteln (Clearingfunktion).

In vergleichbaren Maßnahmen soll vermittelt werden, wie Struktur und Ordnung im Privathaushalt der Maßnahmenteilnehmenden gelingen. Der Ist-Zustand behinderte sowohl die Teilnehmenden als auch die SGB-II-Träger-Verwaltung. Zwar werden nur selten Wohnungen >entmüllt` (zum Beispiel Interview07Regio13), aber beispielsweise benötigte Unterlagen für die Leistungsabteilung gesucht und abgegeben. Aus Sicht der Arbeitsverwaltung ist dieser Zustand in einigen Fällen nur mit Unterstützung von Maßnahmenträgern zu verbessern. Ins- 
gesamt charakterisieren die Befragten die Teilnehmenden als massiv beeinträchtigt in den sozialen, alltagsstrukturierenden Kompetenzen.

\subsubsection{Fallbeispiel einer Maßnahme für Wohnungslose}

Im Aktivcenter nach $\S 46$ SGB III sollen Personen erreicht werden, die meist bei Bekannten, Verwandten oder in Notunterkünften gemeldet sind und Termine beim SGB-II-Träger nicht wahrnehmen. Eine Vermittlung in Arbeit, wie auch eine Verstetigung der Wohnsituation sind intendiert. Für 50 Teilnehmende in drei Gruppen besteht eine offene Maßnahmenstruktur. Dabei verfügt der Maßnahmenträger über weitreichende Handlungsspielräume: Eine Vollzeitteilnahme der Wohnungslosen wird angestrebt, ist jedoch nicht obligatorisch, um die sozial stark isolierte Klientel überhaupt zu erreichen. Insbesondere kreative Angebote sollen die Attraktivität des Maßnahmenbesuchs steigern. Die kreativen Nachmittagsangebote können nur bei Anwesenheit am Vormittag besucht werden.

In der Maßnahme werden an zwei Tagen Frühstückseinheiten, unter fachlicher Begleitung von SozialpädagogInnen, mit einem alltäglichen und lockeren Tagesthema angeboten, um das Gruppenkennlernen und eine Vertrauensbasis zu den Angestellten des Maßnahmenträgers zu entwickeln. Da die Teilnehmenden außerhalb der Maßnahme meist keine regelmäßigen Mahlzeiten einnehmen, erhält hier die angebotene Nahrung eine andere Qualität als dies in anderen Maßnahmen der Fall ist. Bei längerer Maßnahmenteilnahme werden an den Vormittagen Bildungseinheiten durchgeführt, etwa Qualifizierungen zu EDV und Bewerbungsverfahren. Insbesondere zu Maßnahmenbeginn sind die Hauptbestandteile gemeinsames Kochen, gesunde Ernährung mit kleinem Budget, Sporteinheiten (etwa Fußball oder Nordic Walking) und Körperpflege. Sensible Themen wie Hygiene werden zunächst mit einem allgemeinen Vortrag vor der Gruppe eröffnet. Wo Bedarf besteht, finden individuelle Gespräche durch die SozialpädagogInnen statt. Auf Initiative von Maßnahmenteilnehmenden erschien monatlich eine Teilnehmenden-Zeitung, in der Gedichte und kleine Artikel veröffentlicht wurden. Darüber hinaus gibt es die kreativen Nachmittagsangebote (zum Beispiel Malen, CollagenErstellen, Basteln in Serviettentechnik) sowie Theaterprojekte. Die sozialen und kreativen Aspekte der Maßnahme werden vom Sozialpädagogen im Maßnahmenträger als »Das Sprungbrett ins Leben zurück« (Interview03Regio06) bezeichnet. 
Die Klientel ist obdachlos beziehungsweise ohne festen Wohnsitz, jedoch in der Alters-, Geschlechts- und Bildungsstruktur heterogen: Die Spanne reicht von Teilnehmenden ohne Schulabschluss bis zu Personen mit abgebrochenem oder abgeschlossenem Studium. Suchterkrankungen kommen im Laufe der Maßnahme immer wieder zum Vorschein, woraufhin im Rahmen eines Einzelgespräches die Aufnahme einer Therapie angeregt und diese gegebenenfalls begleitend unterstützt wird, um dann nach dem Therapieabschluss die Maßnahme fortzusetzen. Die befragten Maßnahmenträgerbeschäftigten berichten durchgängig, dass viele Personen »schwere Schicksalsschläge« erlebt haben und sich nur bedingt mit ihrer eigenen Lebenssituation auseinandersetzen können. Psychisch Labile seien ebenso vertreten wie Menschen, die sich für ihren Lebensstil bewusst entschieden haben. Der Sozialpädagoge erklärt, dass Teilnehmende teils in stabile soziale Netzwerke einbezogen sind, es gebe jedoch auch Personen, die sich sozial abgrenzen. Dies sei vor dem Hintergrund der Lebensrealität sehr rational: »Es gibt Wohnheime, in denen Gewalt vorherrscht.« (Sozialpädagoge, Interview03Regio06)

Die Maßnahme ziele darauf, Alltagsstrukturen herzustellen und die Personen mit sozialen Basis-Kompetenzen auszustatten, um eine spätere Arbeit mit ihnen in der Bezirkssozialhilfe beziehungsweise im Jobcenter überhaupt zu ermöglichen.

Wohnungslose sind insbesondere durch die fehlende Postadresse für Behörden schwer erreichbar. Darüber hinaus gibt es Personengruppen, die trotz der formal erfolgreichen Zustellung der Einladungen und sogar angesichts der höchsten Sanktionsstufe nicht zu den Terminen im SGB-II-Träger erscheinen. Um diese Personengruppe zu erreichen, wurden Maßnahmen mit aufsuchenden Diensten und rahmenden Angeboten geschaffen, die zur Maßnahmenteilnahme motivieren sollen (Theater-, Tanz- und Musikprojekte). ${ }^{9}$ Teilnehmende mit schwindenden sozialen Kontakten, Rückzugstendenzen bis hin zu sozialer Isolation werden vom SGB-II- und Maßnahmenträgerpersonal beschrieben, doch auch die Angst vor gesellschaftlicher Stigmatisierung durch fehlende Erwerbstätigkeit und der Verdacht, sich in der Erwerbslosigkeit einzurichten, lässt sich den Beschreibungen entnehmen.

9 Ein Beispiel wäre die zunächst als Arbeitsgelegenheit, dann als Aktivcenter angebotene Maßnahme für unter 25-Jährige in Regio12 (Interview01Regio12). 
In einigen Regionen reagiert das Fallmanagement der SGB-II-Träger im Verbund mit den Maßnahmenträgern auf Suchtkrankheiten, indem spezifische Maßnahmen geschaffen werden, etwa für Alkoholabhängige, bei denen eine Problemlagen-homogene Gruppe als Voraussetzung für eine funktionierende Maßnahme angesehen wird. Für Alkoholabhängige solle eine Maßnahme folgendermaßen beschaffen sein:

»Auch Institutionen, Einrichtungen zu haben, wo, wenn der morgens Angst hat, wo er morgens trinken muss, damit er sich wieder beruhigen kann, ruhig mal ne kleine Fahne haben kann. Das darf nicht zur ständigen Einrichtung werden, aber die Ängste zu nehmen. Wenn einer jeden Tag trinkt, der kann gar nicht mehr ohne, der muss langsam sich wieder dran gewöhnen an das normale Leben.« (Fallmanager, Interview02Regio10) ${ }^{10}$

In einer Studie geben 53 Prozent der Grundsicherungsstellen an, dass Bedürfnisse und Problemlagen von Menschen mit Suchttendenzen allgemein berücksichtigt werden, jedoch keine gezielte Förderung in die für diese Personengruppe konzipierten Maßnahmen erfolgt (Henkel/Henke/Nägele/ Pagels/Wagner 2009: 118). Faktisch sind Suchterkrankte in der Gruppe der Arbeitslosen jedoch überproportional häufig vertreten (Oschmiansky 2010).

Insgesamt zeigt sich, dass die aufgefundenen niederschwelligen Maßnahmen Sozialer Aktivierung vielgestaltig, innovativ und bislang kaum standardisiert sind. Überdies entstehen die Maßnahmen meist nicht auf dem Wege der Standardinstrumente, sondern über diverse innovativ-kreative Realisierungswege. Daher werden im Folgenden die Genese und die rechtliche Realisierungsform der Maßnahmen Sozialer Aktivierung im Sample exploriert. Die Rekonstruktion der Entstehung bietet Aufschluss über die institutionellen und arbeitsmarktpolitischen Kontexte der niederschwelligen Maßnahmen.

10 Eine recht ähnliche Position vertritt eine Teamleiterin in Interview02Regio05: Auch ihr geht es vorerst nicht um vollkommene Abstinenz, sie vertritt die Vereinbarkeit von Sucht und Jobcenter- beziehungsweise Integrations-Erfordernissen. 


\subsection{RECHTLICHER UND FINANZIELLER RAHMEN FÜR DIE SCHAFFUNG SOZIAL AKTIVIERENDER MASSNAHMEN}

Um das Phänomen der Maßnahmen Sozialer Aktivierung umfassend verstehen und beschreiben zu können, ist die überblicksartige Darlegung der rechtlichen Rahmenbedingungen von Bedeutung. Für die Exploration der Genese dieser innovativen Maßnahmen sind finanzielle Aspekte sowie institutionelle Bedingungen der Entstehung maßgeblich. Die Rekonstruktion dieses institutionellen und normativen Rahmens fundiert die anschließende Analyse der Maßnahmenfunktionen.

Das gesamte Verfahren des Einkaufs beschäftigungsfördernder Maßnahmen unterliegt europäischen sowie bundesweiten gesetzlichen Regelungen und Anweisungen der Bundesagentur für Arbeit. Bei jeder öffentlichen Auftragsvergabe ist zu prüfen, ob ein europaweites Vergabeverfahren im Sinne der $\S \S 97$ VI, 127 GWB, § 4 I VgV oder das deutsche Vergaberecht nach $\S \S 55$ Bundes- und Landeshaushaltsordnungen $\mathrm{BHO} / \mathrm{LHO}$ angelegt werden muss (Koenig/Haratsch 2003: 2637). ${ }^{11}$ Ferner muss der Bedarf geprüft werden, woraufhin Ausschreibung, Vergabe und rechtliche Realisierungsform der Maßnahme, die Vergabeund Vertragsordnung für Leistungen (VOL) ${ }^{12}$ erfüllen müssen und eine Dokumentation des Vergabeprozesses erfolgen. Weiter sieht $\S 97$ Abs. 1 des Gesetzes gegen Wettbewerbsbeschränkungen (GWB) vor, dass öffentliche Auftraggeber wie die SGB-II-Träger Arbeitsmarktleistungen unter anderem im Rahmen von transparenten und wettbewerblichen Vergabeverfahren vergeben. Dem liegt die Annahme zugrunde, dass durch den Wettbewerb Dienstleistungsaufträge zu den günstigsten Konditionen vergeben werden und die öffentlichen Auftraggeber damit ihrer Pflicht zur sparsamen Haushaltsführung ${ }^{13}$ genügen (Koenig/Haratsch 2003: 2638).

11 Die Vergabeverordnung $(\mathrm{VgV})$ trifft nähere Bestimmungen über das einzuhaltende Verfahren bei der Vergabe öffentlicher Aufträge, die in den Anwendungsbereich nach $\S 2$ dieser Verordnung fallen. Bei einem Auftrag unterhalb 200.000 Euro ist das deutsche Haushaltsrecht mit den Verdingungsordnungen (VOB/A, VOL/A) bindend, oberhalb gilt das EU-Kartellvergaberecht nach GWB, VgV, VOB/A, VOL/A, VOF.

12 Die VOL/A enthält dabei den Kern der materiellen Regelungen zum Vergabeverfahren, unter anderem bezüglich der Prüfung und Wertung von Angeboten sowie der Inhalte der Vergabeunterlagen.

13 Zur Wirtschaftlichkeit und Sparsamkeit sind öffentliche Haushalte unter anderem gemäß Haushaltsrecht nach $\S 30 \mathrm{HGrG}$ verpflichtet. Umgesetzt wird dieser Grundsatz 
Die Maßnahmenplanung muss an Zielvorgaben des Bundesministeriums für Arbeit und Soziales und der Bundesagentur für Arbeit orientiert werden, die je nach Legislaturperiode und politischer Regie des Bundesministeriums unterschiedlich akzentuiert sein können. Die Förderung von Älteren und Jugendlichen sowie arbeitsmarktnahe Förderinstrumente liegen während des Befragungszeitraumes im Fokus der Beschäftigungsförderung (Interview03Regio07, Eppel et al. 2012). Im SGB II sind »Leistungen zur Beendigung oder Verringerung der Hilfebedürftigkeit insbesondere durch Eingliederung in Arbeit« ( $§ 1$ Abs. 3 SGB II) ebenso wie den Lebensunterhalt sichernde Leistungen verankert. Um die Einhaltung der zentralen Zielvorgaben zu gewährleisten, werden mit den Geschäftsführungen der SGB-II-Träger regionale Zielvorgaben ausgearbeitet und zum Beispiel die erreichte Integrationsquote gemessen.

Inwiefern die niederschwelligen Maßnahmen Sozialer Aktivierung in den Zielvorgaben bemessen werden, nach welchen Kriterien solche Maßnahmen in SGB-II- und Maßnahmenträgern konzipiert und realisiert werden und welche weiteren Rahmenbedingungen diesen Prozess begleiten, wird diskutiert, indem Grundzüge der Planung von Maßnahmen der Beschäftigungsförderung dargelegt (Kapitel 5.2.1), typische Entstehungswege Sozialer Aktivierung aufgezeigt (Kapitel 5.2.2) sowie rechtliche Realisierungsformen der Maßnahmen im Sample herausgearbeitet (Kapitel 5.2.3) werden.

\subsubsection{Planung und finanzielle Mittel}

Die Beschaffung der sogenannten Arbeitsmarktdienstleistungen erfolgt innerhalb eines komplexen juristischen Kontextes. Daher unterliegt das Verfahren institutionellen Standardisierungsprozessen und häufig arbeitsteiligem Vorgehen. Von den lokalen SGB-II-Trägern werden die Budgets für beschäftigungsfördernde Maßnahmen geplant und gesteuert. ${ }^{14}$ Auf Ebene der Maßnahmenkoordination werden die von der Bundesagentur für Arbeit zur Verfügung gestellten finanziellen Mittel zu den Maßnahmenbedarfen ins Verhältnis gesetzt. Dieser Prozess

durch das Vergaberecht $\S \S 55 \mathrm{BHO} / \mathrm{LHO}$ und das Zuwendungsrecht $\S \S 23,44 \mathrm{BHO} /$ LHO. Diese Bestimmungen müssen bei der Verausgabung der Mittel durch SGB IITräger, dem sogenannten Eingliederungstitel SGB II für die Beschäftigungsförderung, berücksichtigt werden.

14 Neben den durch die Jobcenter verwalteten Leistungen zur Eingliederung in Arbeit existieren auch EU- und Bundeprogramme wie zum Beispiel 50plus. 
kann von einem dafür zuständigen Team oder einzelnen Beschäftigten durchgeführt werden.

Im Team der Maßnahmenkoordination in Regio02 werden Maßnahmen aus dem SGB III (etwa Eingliederungszuschüsse nach § 46 SGB III Trainingsmaßnahmen) und dem SGB II (etwa Arbeitsgelegenheiten nach § 16d SGB II) eingekauft und administriert. Bewirtschaftet werden auch Eingliederungstitel (Bundesmittel für die Leistungen zur Eingliederung in Arbeit, die gemäß der Eingliederungsmittelverordnung des Bundesministeriums für Arbeit und Soziales auf die jeweiligen Jobcenter aufgeteilt werden):

»Gucken, haben wir noch Geld, haben wir kein Geld, gucken, wo muss man's hinschieben, können wir uns noch was kaufen, können wir noch Maßnahmen fahren, können wir Einstiegsgeld gewähren und solche Sachen. [...] Insofern laufen bei uns auch die Fäden der ARGE bisschen zusammen. Also was Sie vorhin angesprochen haben zu niederschwelligen Maßnahmen, das läuft dann auch bei uns zusammen, weil das muss ja gepflegt werden, das muss ja betreut werden, das muss ja auch fürs Controlling sichtbar gemacht werden« (Teamleiterin, Interview04Regio02)

Das jährliche Budget für Arbeitsmarktdienstleistungen ist deutlichen mittel- bis kurzfristigen Schwankungen ausgesetzt, insbesondere durch unvorhersehbare Etatabhängigkeiten, Gesetzesänderungen und die Erfüllung von Integrationsquoten in den ersten Arbeitsmarkt. Daneben beeinflussen auch die regionale Konjunktur und Arbeitsmarktstruktur die laufenden Maßnahmenbudgets. Diese Unwägbarkeiten werden auf der anderen Seite bezüglich der Maßnahmenauslastung durch Teilnehmende komplettiert. In diesem Kontext vollzieht sich die konkrete Maßnahmenplanung. Bedarfsanalyse und Erfahrungen aus vorherigen Maßnahmen werden etwa über folgende Kommunikationswege der Arbeitsvermittlung und des Fallmanagements berücksichtigt (Interview02Regio04):

1.) Vermittlung und Fallmanagement können sich initiativ mit Maßnahmenideen an ihre Teamleitung und Maßnahmenkoordination wenden. Laut Teamleitung wird diese Möglichkeit auch genutzt: »Also da gibt es durchaus keine Berührungsängste und da sagen die dann auch mal, also das, was ihr da gekauft habt [...], also das brauchen wir nicht mehr.« (Interview02Regio04)

2.) Vermittlung und Fallmanagement sind in den regelmäßigen Planungs- und Kaufprozess der Maßnahmen einbezogen. Die Teams sammeln Maßnahmenideen auf Basis der Kundenstruktur, zunächst unabhängig von der rechtlichen Realisierbarkeit. Diese Vorschläge werden dann bei der Maßnahmenkoordination- und -planung analysiert und Umsetzungsoptionen entwickelt. 
Letztlich wird die Maßnahmenplanung über die Teamleitung mit der entsprechenden Jobcenterleitung abgestimmt. Sich verändernde finanzielle Ressourcen ${ }^{15}$ können ein Nachjustieren der Maßnahmenplanung im laufenden Haushaltsjahr zur Folge haben. In der dünnbesiedelten Regio11 wird die Maßnahmenplanung von einer Teamleiterin, die die Maßnahmen mehrerer SGB-II-Träger-Standorte koordiniert in zwei Phasen eingeteilt (Interview03Regio11): Eine orientierende Planungsphase, in der eine grobe Vorplanung des kommenden Rechnungsjahres erfolgt und eine Phase der Detailplanung. Bei den Kalkulationen werden neben Mittelverwendungsvorgaben auch regionale Faktoren berücksichtigt, zum Beispiel ein erhöhter Fahrtkostenbedarf für Teilnehmende in dünn besiedelten Regionen. Das Maßnahmenbudget in der Regio11 wurde zwei Mal an geänderte Vorgaben angepasst. Diesen Wechsel der Zuweisungen durch die Bundesregierung, der für Verwaltungskosten (Gehälter, Dienstleistungen, Mieten, Nebenkosten) und Eingliederungsleistungen getrennt erfolgt, erklärt die Teamleiterin insbesondere durch Schwankungen bei den Gehaltsaufwendungen der JobcenterAngestellten. Denn die Leistungen zur Eingliederung in Arbeit (Eingliederungstitel Egt2) und die Verwaltungskosten in der Grundsicherung (Verwaltungskostenbudget) sind gegenseitig deckungsfähig ( $\$ 46$ Abs. 1 S.5 SGB II). Das jährliche Austarieren, welche Kosten die Kommune und welche das Jobcenter übernimmt, erkläre die Rückflüsse vom Eingliederungs- in den Verwaltungstopf. ${ }^{16}$ Insgesamt bedarf es hier einer hohen Planungskompetenz. Nachdem die Teamleitung, die mit der Budgetierung betraut ist, den Teamleitern der SGB-IITräger-Standorte die Maßnahmen-Budgets, Teilnahmezahlen, Verweildauer etc. zugewiesen hat, besprechen die lokalen Teamleitungen in ihren Teams, welche Maßnahmen durchgeführt werden sollen (Interview01Regio11).

Ähnlich institutionalisierte Abläufe der Maßnahmenplanung und Budgetierung gibt es auch in den Optionskommunen, wie beispielsweise im Projektmanagement in Regio13. Die dortige Abteilung des Projektmanagers verstehe sich als »Dienstleistungsabteilung fürs Fallmanagement." (Projektmanager, Interview06Regio13): Das Projektmanagement betreut die Maßnahmen und unterrichtet das Fallmanagement über den Verlauf der einzelnen Maßnahmen. Das

15 Etwa verursacht durch die Kooperation mit Kommunen, Haushaltssperren oder das Erreichen der Integrationsquote.

16 Anfang 2011 sah sich die Teamleiterin dazu gezwungen, Gelder aus dem Eingliederungstopf in den Verwaltungstopf zu transferieren, doch sollten diese im Juni 2011 wieder in den Eingliederungstopf zurückfließen. Der Rückfluss wird durch den Einbehalt des Bildungs- und Teilhabepaketes ermöglicht. 
Projektmanagement gleicht die in Gesprächsrunden mit dem Fallmanagement, von Letzterem kommunizierten inhaltlichen Maßnahmenanregungen mit den Gesetzesgrundlagen ab. Nach Klärung potentieller Maßnahmeninhalte wird eruiert, ob der quantitative Bedarf ausreichend vorhanden ist (Interview06Regio13). Optionskommunen, die über entsprechende kommunale Mittel verfügen, installieren teilweise Sonderprogramme zur Beschäftigungsförderung. Die Zuweisung der Bundesmittel zur Beschäftigungsförderung an optierende Kommunen entspricht den Standards für die SGB-II-Träger. In Regio13 wird betont, dass insbesondere durch die Sonderprogramme soziale Schieflagen in diesem SGB-IITräger-Modell stärker entschärft werden könnten (Interview01Regio13). Im Folgenden werden zwei Optionskommunen dargestellt, die erste (Regio13) ohne kommunal finanziertes Beschäftigungsförderungsprogramm und die zweite (Regio06) mit einem Förderprogramm.

In Regio13 gibt es keinerlei zusätzliche kommunale Gelder für die Beschäftigungsförderung. Einzige Ausnahme seien Kooperationsprojekte mit dem Jugendamt. Vom Gesamtbudget (ohne das Grundsicherungs-Budget), das der Leitung Projektmanagement für die Eingliederung zur Verfügung steht, werden Pflichtaufgaben bestritten; so muss im Bildungsbereich für jeden erwerbslosen Jugendlichen eine Maßnahme, Erstausbildung oder Qualifizierung finanzierbar sein. Im von der Interviewten umrissenen Beispiel entfallen die Hälfte des 20 Millionen Euro umfassenden Eingliederungstitels auf den Bildungsbereich, ein Viertel entfallen auf Mobilitätskosten (Umzug, Fahrtkostenzuschüsse) und das übrige Viertel wird dem Beschäftigungsbereich zugewiesen. Aus dem Budget des Beschäftigungsbereiches werden die hier relevanten sozial aktivierenden Maßnahmen finanziert. 2011 stellte die Optionskommune aufgrund des knappen Budgets alle Arbeitsgelegenheiten (AGH) in Entgeltvariante auf die günstigere Mehraufwandsentschädigung um. Der gesamte Landratsamtshaushalt wurde nicht freigegeben, wegen einer überschuldungsbedingten Prüfung. Die gesamte Optionskommune inklusive dem von der Befragten geleiteten Projektmanagement unterliegen zum Zeitpunkt der Erhebung einem Haushaltsstopp.

Demgegenüber wird in der Optionskommune Regio06 ein eigenes Beschäftigungsförderungsprogramm durch die Kommune betrieben. Kommunale Mittel werden insbesondere für die Förderung von SGB-II-Leistungsbeziehenden aus den unteren drei Profillagen verwendet und damit das bestehende Angebot der Arbeitsgemeinschaften ergänzt. Die kommunale Beschäftigungsförderung existiert seit knapp dreißig Jahren, um sozial benachteiligte Gruppen wieder in den Arbeitsmarkt zu integrieren. Früher zielte sie auf Sozialhilfe-, heute auf Alg IIEmpfangende. Die kommunale Beschäftigungsförderung ist untergliedert sich in fünf Teilprogramme mit rund 25 Millionen Euro Gesamtvolumen (Interview02- 
Regio06). Die Steuerung und Auswahl der Projekte sowie die Mittelverwaltung wird vom Kommunalreferat vorgenommen (Interview04Regio06). Für die konkrete Umsetzung der Teilprogramme sind kommunale Beratungsstellen zuständig, die die Jobcenter betreuen. Die Förderung erfolgt nach folgendem Schema: Die Arbeitsvermittlung schickt Arbeitsuchende mit einem Formblatt zur kommunalen Beratungsstelle. Nach einem zwei- oder dreiwöchigen Profiling, ${ }^{17}$ dessen Kosten dem jeweiligen Jobcenter in Rechnung gestellt werden, sendet die Beratungsstelle den Profilingbogen zurück an das Jobcenter. Insofern fließen kommunale Gelder an andere kommunale Einrichtungen. Nach der Ermittlung einer geeigneten Maßnahme und in Absprache mit den Fachkräften im Jobcenter werden die SGB-II-Leistungsbeziehenden dann Maßnahmen des kommunalen Förderprogramms zugewiesen: »Und dann macht er [der SGB-II-Leistungsbeziehende; CF] halt die Maßnahme. Und am Ende, wenn es gut geht, ist er halt in Arbeit vermittelt.« (Kommunaler Angestellter, Interview02Regio06) Andernfalls gelangen die SGB-II-Beziehenden wieder in die Zuständigkeit der Arbeitsvermittlung des SGB-II-Trägers.

Sowohl rein kommunale als auch mit der Bundesagentur gemeinsam verwaltete SGB-II-Träger können Maßnahmen in reglementierten und standardisierten Prozessen ausschreiben. Dem gehen die Bedarfsermittlung und Budgetplanung voraus. Insgesamt besteht ein Vorrang der öffentlichen Ausschreibung ${ }^{18}$ für Dienstleistungen durch Dritte (Maßnahmenträger), doch in begründeten Ausnahmefällen ist ein nicht offenes Verfahren, ein Verhandlungsverfahren oder ein wettbewerblicher Dialog möglich. Es existiert somit ein Interpretations- und Handlungsspielraum, etwa wenn die einzukaufende Dienstleistung eine außergewöhnliche Eignung benötigt und nur von einem beschränkten Kreis an Organisationen ausgeführt werden kann oder der Aufwand eines offenen Verfahrens in einem Missverhältnis zum Wert der Leistung stehen würde (Abschnitt 2, § 3 EG VOL/A). Beschränkten Ausschreibungen und Freihändigen Vergaben ist ein Teilnahmewettbewerb vorzuschalten, was ebenfalls in begründeten Ausnahmen unterlassen werden kann. Seit 2009 konkurrieren im Ausschreibungsverfahren

17 Im sogenannten Profiling werden Fähigkeiten sowie Vermittlungshemmnisse der Arbeitsuchenden beschrieben und kategorisiert (vergleiche Kapitel 4.6.2 »Fördern und Fordern« als Motor der Aktivierung).

18 Maßnahmen, die in der Regel über 500 Euro kosten, können über eine Öffentliche Ausschreibung ( 3 Nr.2 VOL/A), eine Beschränkte Ausschreibung ( 3 Nr.3,4 VOL/A) oder eine Freihändige Vergabe ( $\$ 3$ Nr.5 VOL/A) gekauft werden. 
gemeinnützige Einrichtungen gleichgestellt mit gewerblichen Bietern. ${ }^{19}$ Abweichungen vom standardisierten Ausschreibungsverfahren sind also möglich, jedoch aufwändiger zu realisieren und zu begründen (unter Berufung auf Sondertatbestände oder Ermessensspielräume).

Zusammenfassend ist festzuhalten, dass der Einkauf beschäftigungsfördernder Maßnahmen in einem komplexen juristischen und institutionellen Rahmen erfolgt. Standardisierungen des Vergabeprozesses oder der Maßnahmeninhalte vermögen die Komplexität zu reduzieren, doch sind innovative Vorgehensweisen wie Maßnahmen Sozialer Aktivierung weiterhin möglich. Voraussetzung ist ein zum Teil weitreichender innerinstitutioneller Abstimmungsprozess, um nicht-standardisierte Maßnahmen zu realisieren. Denn es gilt, innovative Ideen in die umfassenden rechtlichen und institutionellen Regelungen einzubetten. Einzukaufende Beschäftigungsförderungsinstrumente durch Dritte unterliegen grundsätzlich dem Wettbewerbsprinzip und können nur in begründeten Ausnahmen frei vergeben werden. Zudem unterliegen sie Vorgaben der Bundesagentur für Arbeit beziehungsweise arbeitsmarktpolitischen Weisungen, die sowohl die finanziellen Ressourcen der Beschäftigungsförderung prägen als auch deren Verwendung durch Zielvorgaben steuern. Kommunal verwaltete SGB-II-Träger wenden unter Umständen zusätzliche finanzielle Ressourcen für eigene Beschäftigungsförderungsinstrumente auf. Bedingt durch den zusätzlichen Charakter können jedoch auch kommunale Einsparungsmaßnahmen reduzierend oder gänzlich unterbindend wirken. Maßgeblich für die Mittelvergabe im sogenannten Eingliederungstitel sind in der Regel Eingliederungsquoten.

\subsubsection{Entstehungswege der Maßnahmen Sozialer Aktivierung}

Aufgrund des komplexen rechtlichen und institutionellen Kontextes, ist die Genese innovativer Maßnahmen erkenntnisträchtig, insbesondere da keine standardisierten Formalia zu Beginn der Entstehungsphase bestanden. Im Feld lassen sich zwei grundsätzliche Entstehungswege für Maßnahmen Sozialer Aktivierung feststellen: Die Entstehung auf Initiative 1.) des Maßnahmenträgers oder 2.) des SGB-II-Trägers. Wünsche und Anregungen von Erwerbslosen für Maßnahmen können über die Beschäftigten der SGB-II- und Maßnahmenträger in die Maßnahmengenese einfließen, haben hier jedoch einen marginalen Stellenwert, da sie

19 Dem ging die Änderung der VOL/A von 2006 in der Fassung von 2009 voraus, indem $\S 7$ Nr.6 gestrichen wurde. 
allenfalls indirekt über die vorgenannten Institutionenvertretungen wirken können. Im Sample wurde keine Maßnahmenentstehung ausgehend von (potentiellen) Maßnahmenteilnehmenden gefunden, was ein Fallmanager in Regio10 (Interview01) unter anderem mit einer wenig initiativen und wenig motivierten Klientel begründet. Allerdings werden etwaige Wünsche der Erwerbslosen zu neuen Maßnahmen nicht standardisiert erhoben und in die Maßnahmenplanung einbezogen. In Ausnahmefällen kann soziale Aktivierung auf Initiative Dritter über SGB II- und Maßnahmenträger eingeführt werden. Im Sample findet sich beispielsweise eine Tanzmaßnahme, zu der ein Choreograph einen bundesweiten Maßnahmenträger in Regio12 innervierte. ${ }^{20}$ Die Initiative des SGB-II-Trägers kann beinhalten, dass dieser die Maßnahmenimplementierung selbstständig durchführt oder die Ausschreibung und Prüfung über das Regionale Einkaufszentrum der Bundesagentur für Arbeit vorgenommen wird. Eine Sonderrolle nehmen dabei SGB-II-Träger ein, die als Optionskommune aufgestellt sind. Typische Entstehungswege sozialer Aktivierung sind von Bedeutung, da es sich um eine neuartige Maßnahmenform handelt und hier zum Teil zahlreiche Umwege und innerinstitutionelle Hürden überwunden werden müssen. Möchte ein Jobcenter eine Maßnahme Sozialer Aktivierung umsetzen und verfügt über die benötigten Mittel, werden Ideen für Maßnahmeninhalte und/oder die Zielgruppe identifiziert. Dies geschieht meist zunächst intern in Teamsitzungen oder in Abstimmung mit eigenen Projektmanagements. Nach interner Bedarfsfeststellung beginnt der Prozess der Maßnahmenbeschaffung. Dieser differiert jedoch im Sample. Wie in Abbildung 3: Verhältnis in der Maßnahmendurchführung dargestellt, trägt die Verantwortung für Inhalt und Wirtschaftlichkeit einer Maßnahme immer der jeweilige SGB-II-Träger (Jobcenter/Arbeitsgemeinschaft oder Optionskommune). Wird das zuständige Regionale Einkaufszentrum genutzt, ist dieses für die bedarfsgerechte, vergaberechtskonforme und wirtschaftliche Beschaffung im Rahmen von wettbewerblichen und damit transparenten Vergabeverfahren $^{21}$ zuständig und übernimmt, wie in den nachfolgenden Ausführungen dargestellt, damit eine Dienstleistungs- sowie Controllingfunktion.

20 Vergleiche Kapitel 5.2.2.1 Maßnahmenträger als Initiatoren.

21 Dies beinhaltet Öffentliche Ausschreibungen, Beschränkte Ausschreibungen und Freihändige Vergaben. 
Abbildung 3: Verhältnis in der Maßnahmendurchführung

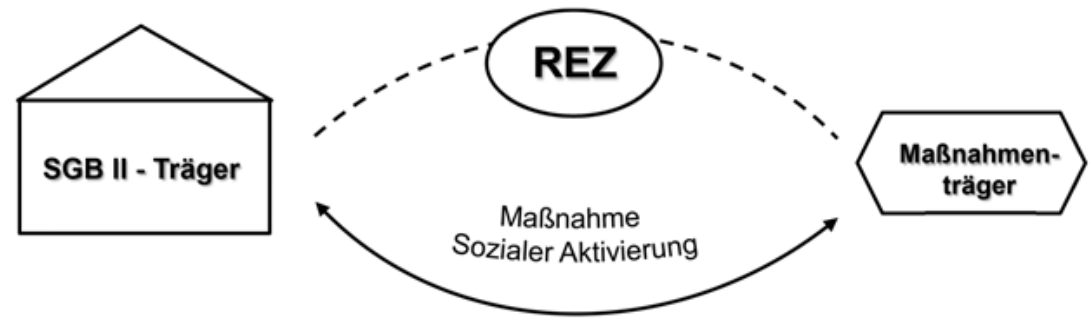

Quelle: Eigene Darstellung

Unabhängig davon, ob die Preisverhandlungen, Zielvorgaben, Einkauf und Administration in den Jobcentern respektive Optionskommunen geschehen oder auf das Regionale Einkaufszentrum übertragen werden, können die eingekauften Maßnahmen von der lokalen Arbeitsvermittlung und dem Fallmanagement mit erwerbslosen SGB-II-Leistungsbeziehenden besetzt werden.

\subsubsection{Maßnahmenträger als Initiatoren}

»Wir sind ja tatsächlich in Regio12 die Initiatoren [für die musisch-sportlichen Maßnahmeninhalte; $\mathrm{CF}$ ] und das haben echt viele [andere Bildungsträger; $\mathrm{CF}$ ] abgekupfert.« (Maßnahmenträgerleitung, Interview04Regio12)

Um die Sonderrolle eines Dritten einfließen zu lassen, wird im Folgenden die Genese einer Tanzmaßnahme dargestellt. Abgesehen vom anfänglichen Wirken eines Choreografen, entspricht diese Maßnahme dem üblichen Weg für die Maßnahmenträgerinitiative. Der überregionale Bildungsträger, der in seiner Selbstbeschreibung zunächst mit herkömmlichen Bildungsmaßnahmen ${ }^{22}$ erfolgreich in Regio12 war, begann 2005 mit neuartigen Maßnahmen, die Tanzkurse für Laien mit abschließender Aufführung beinhalteten. Das musisch-sportliche Angebot wurde daraufhin sukzessive ausgebaut. Begünstigend für die Entstehung war, dass die Kommunalverwaltung in Regio12 sich 2005 verstärkt Jugendlichen als Hauptgruppe zuwandte und vergleichsweise viele Mittel für die

22 Ziel der Standardmaßnahmen waren das Nachholen des Haupt- respektive des Realschulabschlusses (teilweise in Verbindung mit einer AGH), Deutschkurse im Rahmen einer AGH und eine Hinführung zur Erstausbildung. Seit 2005 ist die von der Befragten geleitete $\mathrm{gGmbH}$ in Regio12 der größte Anbieter von Jugend-AGHs. Von den etwa 1000 Teilnehmenden in 2011 sind etwa zwei Drittel Jugendliche. 
Beschäftigungsförderung zur Verfügung stellte. ${ }^{23}$ Auf Initiative eines Dritten wurde eine Maßnahme mit Tanzvorführung geschaffen. Ein berühmter Choreograf wandte sich an einen Maßnahmenträger, da er junge Männer ohne Schulabschluss erreichen wollte, um mit diesen eine Choreografie einzustudieren. $\mathrm{Ab}$ 2007 wurde der von der Interviewten geleitete Träger zum Veranstalter dieses Performance-Projektes in Regio12. Von den aus 200 Personen ausgewählten 40 interessierten männlichen Jugendlichen blieben 2005 circa 25 Projektteilnehmer. Drei Wochen probten die Jugendlichen, um dann im Schauspielhaus von Regio12 die Tanzvorführung gemeinsam mit anderen Benachteiligten-Gruppen zu präsentieren. Das Konzept wird seither unter Regie des Maßnahmenträgers mit ein oder zwei Choreografien jährlich fortgesetzt. Künstlerisch werden Themen wie das schnelle Vergehen der Zeit, Vereinsamung, männliche Geschlechterstereotype oder Liebe bearbeitet. Ab 2008 etablierte der bundesweite Träger regelrechte Tanzklassen und verankerte ein breites Spektrum an sportlich-gesundheitlichen oder künstlerischen Angeboten: Nordic Walking, Laufen, Workout, Rückenschule, Boxen, Fitness, Klettern, Karate, zeitgenössischer und Ausdrucks-Tanz, Jiu-Jitsu, Pilates, Hip-Hop, Musik-Workshops, Theater (in Kooperation mit einer Musicalschule). Wenn also die Tanzangebote den Nukleus bilden, so ist inzwischen eine Bandbreite anderer sportlich-künstlerischer Angebote hinzugetreten. Ferner sind die Maßnahmen auf Personen mit Suchthintergrund, psychischen und physischen Handicaps und Haftentlassene ausgedehnt worden. Es zeigt sich deutlich, dass in Regio12 ein heftiger Konkurrenzkampf der Maßnahmenträger um erwerbslose Jugendliche besteht, die in der Regio12 besonders intensiv gefördert werden. ${ }^{24}$ Der bundesweite Bildungsträger hat ein musikalisch-sportliches Profil entwickelt und bewirbt sich auf Ausschreibungen des Regionalen Einkaufszentrum und sämtlicher in Regio12 relevanten Jobcenter.

23 Vergleiche auch Interview03Regio12: Für die häufige Vergabe der Aktivcenter für Jugendliche war eine Vorgabe der Regio12-übergreifenden Jobcenter-Geschäftsführung für 2010 ursächlich. Aufgrund der guten regionalen Finanzlage war es erklärtes Ziel, alle arbeitslosen Jugendlichen binnen Quartalsfrist in Beschäftigung oder Ausbildung zu bringen. Demnach durfte keine Jugendliche und kein Jugendlicher länger als drei Monate arbeitslos sein, wobei Personen in Maßnahmen weiterhin dem Status arbeitslos innehatten.

24 Der hier interviewte Maßnahmenträger bewirbt massiv und teilweise manipulativ seine Angebote im Bereich U25. So wird in Flyern etwa der Sport- und Tanzanteil besonders stark in den Vordergrund gerückt. Dieses Vorgehen erklärt sich wohl auch damit, dass hier eine schwer zu erreichende Klientel motiviert werden soll. 
Gleichzeitig werden auch initiativ Maßnahmenkonzepte an die Kostenträger herangetragen und eruiert, ob ein neuartiges Maßnahmenangebot tragfähig erscheint. Denn letztlich konkurrieren meist die von einem Maßnahmenträger angeregten Ideen zur sozialen Aktivierung mit Maßnahmenkonzepten anderer Maßnahmenträger in Ausschreibungen des SGB-II-Trägers respektive des jeweiligen REZ. Ein Austausch zwischen Maßnahmenträger und SGB-II-Träger kann so den Gestaltungsrahmen ausloten. Auch in Regio04 kommt es zu einem intensiven Austausch zwischen Maßnahmenträgern und den SGB-II-Trägern: »Der Anstoß kommt von uns selbst, kommt aber auch von [den Maßnahme-; CF] Trägern.« (Bereichsleiter, Interview01Regio04) Die Geschäftsführerin eines christlichen Trägers (Interview03Regio04) sowie weitere Maßnahmenträger treffen sich des Öfteren mit dem Bereichsleiter des SGB-II-Trägers, um über neue Maßnahmen nachzudenken.

Abbildung 4: Initiative zu Sozialer Aktivierung durch den Maßnahmenträger im Maßnahmeneinkauf

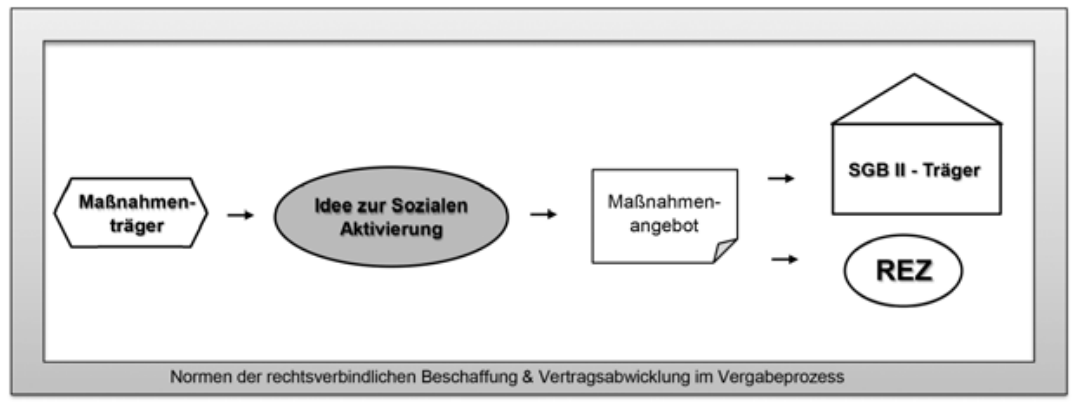

Quelle: Eigene Darstellung

Zusammenfassend bleibt festzuhalten, dass innovative Instrumente, die von den Standardmaßnahmen abweichen, kommunikative Prozesse benötigen. Denn innovative Instrumente können dem innovierenden Maßnahmenträger Wettbewerbsvorteile einbringen oder durch ihre langfristige Dimension der Erwerbsintegration stattdessen den Zuschlag verhindern. Auch der Prozess des Austausches über Angebotsideen zwischen Maßnahmen- und SGB-II-Trägern bewegt sich in einem rechtlichen Rahmen, der die Bedingungen einer rechtsverbindlichen Beschaffung und Vertragsabwicklung garantieren muss. In den meisten hier untersuchten Maßnahmen Sozialer Aktivierung, die dem Typ des Maßnahmenträgers als Initiator entsprechen, war neben der Ausschreibung auch ein direkter Austausch zwischen Maßnahmen- und SGB-II-Träger möglich. Dies wur- 
de durch die Realisierungsform der Arbeitsgelegenheit gefördert, in der die Vergabe in Eigenregie des SGB-II-Trägers erfolgen kann. Auskünften der SGBII- und Maßnahmenträger-Fachkräfte folgend, hat sich die Klientel der Beschäftigungsförderung gewandelt: sie ist arbeitsmarktferner geworden. Dies mache eine Anpassung der beschäftigungsfördernden Instrumente an die arbeitsmarktferne Klientel notwendig, wofür eine Abstimmung zwischen den Maßnahmenanbietern und den SGB-II-Trägern bedeutsam sei, um die Instrumentenanpassung auch im bestehenden rechtlichen Rahmen zu gewährleisten.

\subsubsection{SGB-II-Träger als Initiatoren}

Ein Jobcenter kann unter bestimmten Voraussetzungen (reglementiert durch VOL und weitere rechtliche Auflagen) Maßnahmen autonom vom zuständigen Regionalen Einkaufszentrum ausschreiben und einkaufen. Bisweilen wurde in solchen Fällen geschildert, dass vor oder nach der Ausschreibung ein Informationsaustausch mit Maßnahmenträgern stattfand, in dem bereits Ideensammlungen und Konkretionen gewünschter Maßnahmen vorgenommen wurden. Die Vorbesprechung mit mehreren Trägern könne zur Präzision der Maßnahmengestaltung und stärkeren Passgenauigkeit der Angebote beitragen. Die Angebote würden dann intern geprüft und schließlich dem Angebot der Zuschlag erteilt, das die meisten Punkte in der Gesamtbewertung eines hierfür etablierten Vergabesystems erreicht. Das komplexe und in seiner Durchführung auf Objektivität bedachte Beurteilungssystem ${ }^{25}$ orientiert sich dabei an den Zielvorgaben und dem Bedarf des SGB-II-Trägers. Letztlich lässt jedoch auch dieses Beurteilungssystem einen gewissen Interpretationsspielraum zu und benötigt schon vor der Beauftragung der REZ eine sichere und detailreiche Vorstellung des SGB-IITrägers, wie eine passgenaue Maßnahme aussehen könnte.

25 Laut den REZ-Informationen werden zunächst die Konzepte im Rahmen vorgegebener Detailpunkte in vier Bewertungsstufen mit 0-3 Punkten im Verhältnis zur Passung auf die Anforderungen des bietenden SGB II-Trägers beurteilt. Die Gesamtsumme der sogenannten Leistungspunkte wird durch den Angebotspreis für das Eingliederungshonorar geteilt und mal 100 genommen. Im zweiten Schritt wird ein Kennzahlkorridor auf Basis des führenden Angebotes entwickelt und die Kennzahl minus 10 Prozent errechnet. Im dritten Schritt werden alle in diesem Korridor befindlichen Angebote als gleichwertig betrachtet. Entscheidungskriterium seien die höchste Leistungspunktzahl sowie spezifische Wertungskriterien. Der nach dieser Vorgehensweise wirtschaftlichste Maßnahmenträger erhält dann den Zuschlag. 
In Regio03 wurde beispielsweise, ausgehend von der Beobachtung eines Fallmanagement-Teamleiters, dass eine gewisse Klientel nicht in den Arbeitsmarkt zu vermitteln sei und auch nicht in arbeitsmarktnahe Maßnahmen passe, eine Maßnahme entwickelt, die verschiedene Ebenen der Persönlichkeitsentwicklung adressiert (Interview03Regio07).

»Einer, dem die zwei Schneidezähne fehlen, der kann sich zwar vorstellen, den kannst du auch Bewerbungstraining machen lassen und IT-Systeme erlernen lassen, das bringt nichts. Das war so der Aufhänger und dann haben wir gesagt, ok, wir versuchen eine Maßnahme zu machen, es gibt ja nicht nur zahnlose Leute, es gibt nicht nur Leute, die sich nicht waschen, sondern es gibt Leute, die einfach vereinsamt sind und die es schwer haben und wo man sieht, übergewichtig, also adipös, oder irgendwie schon von außen hin schon Hemmnisse aufzeigen, wo der Fallmanager sagt, wer kommt denn da, da werde ich ja die nächsten 20 Jahre gar nicht mehr quitt.« (Zentraler Projektmanager; Interview03Regio07)

Die Maßnahme entstand in einer Phase, in der noch vergleichbar viele Haushaltsmittel, relativ viele arbeitsmarktnahe KlientInnen gemeldet und die Vermittlungsquoten sehr gut waren. Um weiterhin erfolgreiche Quoten generieren zu können und nicht nur die arbeitsmarktnahe Klientel >abzuschöpfen` (Creaming), wurde die Strategie verfolgt, »Personen von unten nach oben zu holen« (zentraler Projektmanager, Interview03Regio07). Für diesen Zweck wurden zuerst die vorhandenen Arbeitsgelegenheiten genutzt, die überwiegend durch praktische Tätigkeiten geprägt waren und in denen keine gezielte Bearbeitung bestehender Hemmnisse notwendig war. Die Initiatoren verzichten aufgrund des äußeren Erscheinungsbildes der Teilnehmenden bewusst auf den Einsatz in sensiblen Bereichen wie Kindertagesstätten oder Seniorenheimen.

»Da haben wir erkannt, eine [arbeitsmarktnahe; CF] AGH löst die Probleme nicht, sondern wir müssen an die Persönlichkeit heran und nicht an das Berufsfeld und dann haben wir das erst einmal ausgeschrieben und gesagt, da gehört jetzt zuerst einmal eine Persönlichkeitsentwicklung dazu und was haben die Leute denn, und dann haben wir eine Liste gemacht. Also, die haben im Regelfall Schulden, die haben eine Suchtmittel-Problematik, die haben eine Übergewichts-Problematik, die haben eine Magersucht, die haben Probleme überhaupt im zwischenmenschlichen Bereich, die haben Probleme der Gruppenfindung, die Probleme der Teamfähigkeit, so Teamfähigkeit in sehr weitem Sinne, das sind Vereinsamungstendenzen, Sozialisationsprobleme und neben anderen Geschichten, die kamen aus dem Knast, und konnten deshalb sich vielleicht nicht zurecht finden, die hatten Sprachprobleme und und und. So, und die meisten hatten vieles und keine Ahnung von Beruf und keine Ahnung von Theorie und Schule und jahrelang arbeitslos, und das haben 
wir zusammengefasst und dann haben wir die Maßnahme ausgeschrieben« (zentraler Projektmanager, Interview03Regio07).

Bereits in der Ausschreibung wurden sozial aktivierende Eckpunkte der gewünschten Maßnahme seitens des SGB-II-Trägers thematisiert, doch auch nachdem der Maßnahmenträger mit der stärksten Passung den Zuschlag erhalten hatte, wurde ein intensiver Austausch betrieben, um die benötigten Leistungen zu garantieren: »Das ist der Standard, den müsst ihr machen, wir verlangen von Euch aber eigentlich mehr.« (Zentraler Projektmanager, Interview03Regio07) Der Maßnahmenträger stimmte der Erweiterung zu, wollte zunächst mit den Grundmodulen starten und die Angebote schrittweise erweitern. Diese direkte Kommunikation mit dem Maßnahmenträger während der laufenden Maßnahme tritt insbesondere dann auf, wenn, wie in Abbildung 5: Maßnahmeneinkauf durch den SGB-II-Träger dargestellt, der Einkaufsprozess in der Verantwortung des SGB-II-Trägers liegt.

\section{Abbildung 5: Maßnahmeneinkauf durch den SGB-II-Träger}

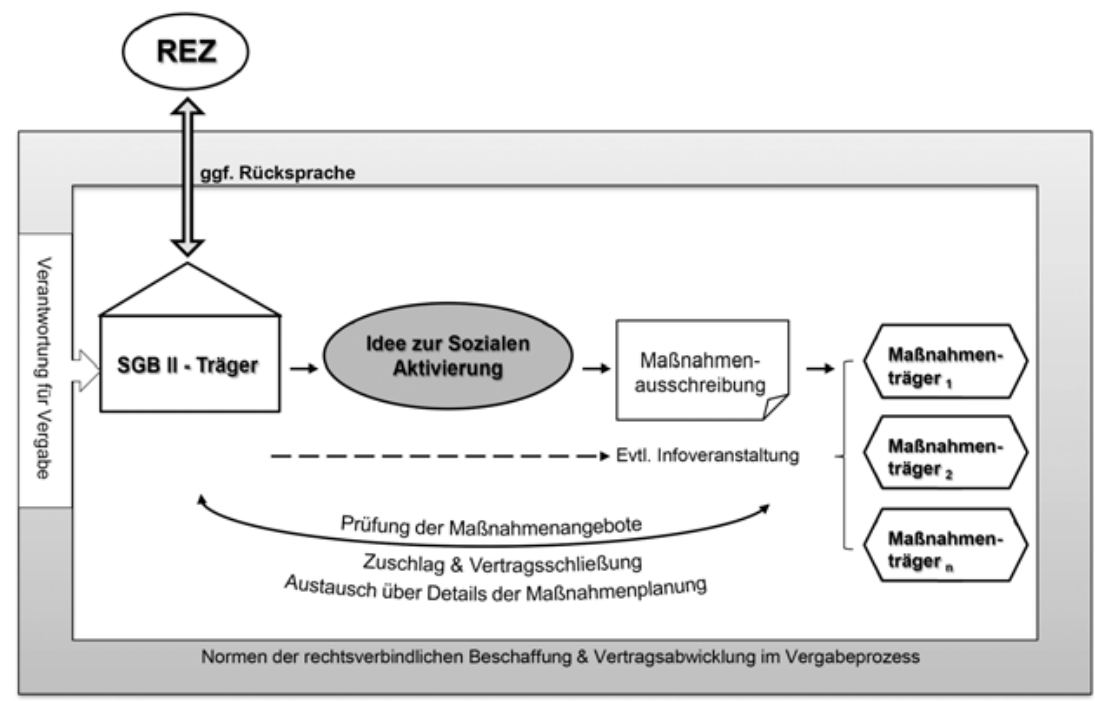

Quelle: Eigene Darstellung

Der SGB-II-Träger in Regio07 ist ein prägnantes Beispiel dafür, dass viele SGBII-Träger vor allem spezifische Zielgruppen im Blick haben, die über eine niederschwellige Maßnahme erreicht werden sollen. Die Idee Sozialer Aktivierung muss in eine konkrete Maßnahmenausschreibung einfließen, die auch Rahmen- 
vorgaben über die inhaltlichen Ziele der Maßnahmengestaltung ermöglicht. Hier kann entweder im Alleingang eine Maßnahmenausschreibung erfolgen oder eine vorkoordinierende Informationsveranstaltung für Maßnahmenträger angesetzt werden. Im Rahmen von Arbeitsgelegenheiten, zertifizierten Bildungsmaßnahmen (FBB), Pilotprojekten und dem Vermittlungsbudget kann der SGB-IITräger in Eigenregie ausschreiben und verantworten. In solchen Fällen kann der SGB-II-Träger allerdings auch eine Rechtsberatung durch das zuständige REZ einholen. Diese Bedingungen sind für das Einbringen von innovativen Elementen wie der sozialen Aktivierung besonders günstig. Weiter sind Möglichkeiten einer direkten Kommunikation zwischen SGB-II- und Maßnahmenträger auch im Maßnahmenverlauf hilfreich, um auf zuvor unbekannte Voraussetzungen der Teilnehmenden reagieren zu können.

Die Maßnahme in Regio07 wurde 2006 über $\S 37$ SGB III (Beauftragung Dritter mit der Vermittlung) mit einem Bewerbungscenter und der Heranführung an die Beschäftigungsfähigkeit mit $90 \mathrm{Maßnahmenplätzen} \mathrm{eingeführt.} \mathrm{Mittler-}$ weile läuft die Maßnahme als Aktivcenter im Rahmen des § 46 SGB III. Für den Maßnahmenträger sind die Bedingungen seit der Wandlung von der Beauftragung Dritter hin zu einem Aktivcenter mit einer größeren Konkurrenz anderer Maßnahmenträger verbunden, da sich die Maßnahme etabliert hat: »Dann müssen sie automatisch [...] einen anderen Preis anbieten, um weiter im Rennen zu bleiben« (Maßnahmenträger-Standortleiterin, Interview04Regio07). Außer einer knapperen Mittelausstattung hat sich durch die geänderte Rechtsgrundlage der $\mathrm{Ma}$ ßnahme nichts geändert. Dabei seien die individuellen Zuweisungszahlen je Fallmanager sehr hoch, die Maßnahme gilt als »Grundrüstzeug des Fallmanagements« (zentraler Projektmanager, Interview03Regio07).

Mit dieser Maßnahme ist die Einführung eines innovativen Instruments geglückt, gemessen an der Akzeptanz durch die SGB-II-Träger-Fachkräfte. Doch der zentrale Projektmanager erklärt, dass mittlerweile aufgrund der geringeren Mittel Kofinanzierungen zunehmend an Bedeutung gewinnen. In von der Kommune kofinanzierten Maßnahmen könnten kommunale Eingliederungsleistungen nach $\S 16 a$ SGB II ${ }^{26}$ eingesetzt, und damit Leistungen angeboten werden, die die zentrale Steuerung in Nürnberg blockiere. Geplant sind (weitere) kofinanzierte

26 Zur Verwirklichung einer umfassenden Betreuung und Unterstützung können folgende Leistungen, die für die Eingliederung der oder des erwerbsfähigen Leistungsberechtigten in das Erwerbsleben erforderlich sind, erbracht werden: die Betreuung minderjähriger oder behinderter Kinder oder die häusliche Pflege von Angehörigen, Schuldnerberatung, psychosoziale Betreuung, Suchtberatung. 
Maßnahmen mit psychosozialer Betreuung und Schuldnerberatung unter einem Dach, die über Mittel der Bundesagentur, des Europäischen Sozialfonds und der Kommune finanziert werden. Der zentrale Projektmanager würde eine solche Maßnahme nur mit der Rückendeckung des Regionalen Einkaufszentrums durchführen, da andernfalls der verantwortlichen SGB-II-Träger-Fachkraft ein Haftungsverfahren drohen könnte. ${ }^{27}$ Doch in der Praxiserfahrung zeige sich, dass Schuldner-, Suchtberatung, psychosoziale Betreuung, und die Betreuung minderjähriger oder behinderter Kinder sowie die häusliche Pflege von Angehörigen insbesondere im arbeitsmarktfernen SGB-II-Klientel, bedeutsam seien und die Maßnahmendurchführung erleichtern würden.

Das Modell des Maßnahmeneinkaufs weist in Optionskommunen die gleichen Mechanismen auf, wie der Einkauf der Jobcenter/Arbeitsgemeinschaften. Die Optionskommunen sind jedoch, im Unterschied zu Jobcentern, direkt der Landrätin beziehungsweise dem Landrat unterstellt. Allerdings verfügen Optionskommunen über einen stärkeren Ermessensspielraum bei der Frage, welche Maßnahmen über das Regionale Einkaufszentrum eingekauft oder intern abgewickelt werden können (Interview06Regio13). Höhere Handlungsspielräume im Vergleich zu Jobcentern aus lokalen Kooperationen von Arbeitsagenturen und Kommunen $\mathrm{zu}$ erreichen, ist jedoch von den kommunalen Mittelzuweisungen beeinflusst. Der Teamleiter des SGB-II-Trägers in der finanziell gut ausgestatteten Regio06 kauft hauptsächlich Standardmaßnahmen über das REZ nach dem üblichen Verfahren ein. Die kommunal finanzierten Maßnahmen beinhalten jedoch eine Bandbreite an innovativen Elementen, die zusätzlich zu arbeitsmarktnäheren Elementen wie Bewerbungstraining, EDV- und Englischkurse. Folgende von der Kommune finanzierte Maßnahmen bietet die kommunale Beschäftigungsförderung:

27 Die Geschäftsführung in Regio07 ist der Kofinanzierung gegenüber äußerst aufgeschlossen, und auch weitere regionale Jobcenter würden solche Maßnahmen eventuell als gemeinsames Projekt anbieten wollen. Doch die Rückmeldung ist: »Nürnberg ist noch nicht so weit und wir sind da noch in der Erprobung« (zentraler Projektmanager, Interview07Regio03). Die Standortleiterin des Maßnahmenträgers erklärt, dass sich der Träger bereits für eine solche aus ESF-Mitteln und aus Mitteln der Bundesagentur kofinanzierte Maßnahme beworben habe, diese jedoch aufgrund fehlender Mittel der Bundesagentur nicht realisiert werden konnte (Interview04Regio07). 
1.) Arbeitsgelegenheiten im Kunstbereich (Entwicklung eines Kunstprojektes)

2.) Qualifizierungs- und Bewerbungstrainings

3.) Kurse für Migrantinnen (zum Beispiel Sprachtraining, Buchhaltung)

4.) Maßnahme für Alkoholkranke und Planung einer Maßnahme für Drogenabhängige

5.) Arbeit an der Hygiene

Es existieren diverse kommunale Projekte für eine Klientel mit mehreren Vermittlungshemmnissen, die in einer Bedarfsgemeinschaft oder in einem Singlehaushalt leben, zum Beispiel eine Maßnahme für Wohnungslose. ${ }^{28}$ Niederschwellige Bestandteile in anderen Maßnahmen sind zudem Gesundheitsberatung und ein Theaterworkshop im Rahmen eines Alleinerziehenden-Projektes. In der wissenschaftlichen Zusammenschau wird deutlich, dass in Regio06 ein im Vergleich zur Optionskommune in Regio13 sehr weitreichendes zusätzliches Maßnahmenportfolio angeboten wird. In der regionalen Kooperation einer Optionskommune mit einer Arbeitsgemeinschaft ist es jedoch problematisch, so die kommunale Fachbereichsleiterin (Interview04Regio06), dass die Kommune von den Zuweisungen durch die ARGE abhängig ist. So könnten Großmaßnahmen anderer Maßnahmenträger der Arbeitsgemeinschaft primär beschickt werden, zu Ungunsten der Maßnahmenauslastung der kommunalen Beschäftigungsförderung. Dem ließe sich in analytischer Perspektive die Sicht der Bundesagentur für Arbeit ergänzen, wonach die Maßnahmengestaltung der Optionskommune nicht die Zuweisungspraxis des Jobcenters bestimmen darf.

Zusammenfassend ist festzuhalten, dass bei Entstehung, Durchführung und Nachlese einer Maßnahme ein intensiverer Austausch zwischen SGB-II- und Maßnahmenträger besteht, wenn der SGB-II-Träger die Maßnahme in Eigenregie vergibt. Dieses Alleinstellungsmerkmal der SGB-II-Träger-initiierten Maßnahmen kann die Entstehung von Maßnahmen Sozialer Aktivierung begünstigen, wenn eine arbeitsmarktferne oder noch nie im SGB-II-Träger vorstellige Klientel angesprochen werden soll. Hier ist eine stärkere `Durchdringungstiefe wahrscheinlich. Solche Maßnahmen dienen auch der Strategie, diese Klientel »von unten nach oben zu holen« (zentraler Projektmanager, Interview03Regio07). Die Analyse der Experteninterviews verdeutlicht den wenig überraschenden Befund, dass eine bereits weitreichend erfüllte Integrationsquote und ausreichende Mit-

28 So zum Beispiel in Interview01Regio06 und Interview03Regio06. 
telkapazitäten sich positiv auf den Einsatz von niederschwelligen Maßnahmen auswirken. Dennoch werden in Phasen knapper Mittel Kooperationen zwischen den Kommunen, Jobcentern und Anbietern sozialer Leistungen ebenfalls notwendig sein, um effektiv bearbeiten zu können.

\subsubsection{Einkauf von Arbeitsmarktdienstleistungen durch das REZ}

Die Regionalen Einkaufszentren (REZ) wickeln im jeweiligen Bezirk als Stützpunkt-Regionaldirektion (RD) die Einkaufsprozesse für Arbeitsmarkt-Dienstleistungen der Agenturen und SGB-II-Träger ab. Die REZ beraten SGB-II-Träger bei der Bedarfsanalyse, erarbeiten mit den SGB-II-Trägern individuelle Leistungsbeschreibungen und Vergabeunterlagen, führen die Vergabeverfahren durch, begleiten den fachlichen Bewertungsprozess der SGB-II-Träger, erteilen Zuschläge, schließen Verträge ab und managen die Vertragseinhaltung. Bundesweit existieren im Erhebungszeitraum fünf Regionale Einkaufszentren. Dem Einsatz der REZ geht eine Beauftragung durch die lokalen Agenturen für Arbeit und SGB-II-Träger voraus. Die Einkaufsorganisation ist in das Service-Haus der Bundesagentur für Arbeit Nürnberg eingebunden. Die fachliche Steuerung der Regionalen Einkaufzentren legt Standards fest, damit alle Verfahren bundesweit einheitlich verlaufen und das Vergaberecht ordnungsgemäß eingehalten wird. In der Steuerung werden die inhaltlichen Vorgaben der Zentrale mit den von den Regionalen Einkaufszentren formulierten Vergabeunterlagen abgestimmt. Hier werden etwa einheitliche Bewertungsschemata und Vertragsmodalitäten erarbeitet. Im Selbstverständnis des für Regio02 zuständigen REZ ist das Ziel des Regionaleinkaufs

»ein wissenschaftlicher und qualitativ hochwertiger Einkauf von Arbeitsmarktdienstleistungen unter Beachtung der bedarfsträgerspezifischen Anforderungen, vergaberechtskonformer Durchführung und Beschaffungsverfahren und bedarfsträgerorientierter Vertragsabwicklung und -betreuung.« (REZ Regio02 o.J.)

Für die Bearbeitung des Maßnahmeneinkaufs durch das REZ kann 1.) die Auswahl des durchführenden Maßnahmenträgers über das Jobcenter oder 2.) die Ausschreibung und Vergabe über das REZ erfolgen. 
Abbildung 6: Maßnahmeneinkauf über das REZ mit Auswahl des SGB-IITrägers

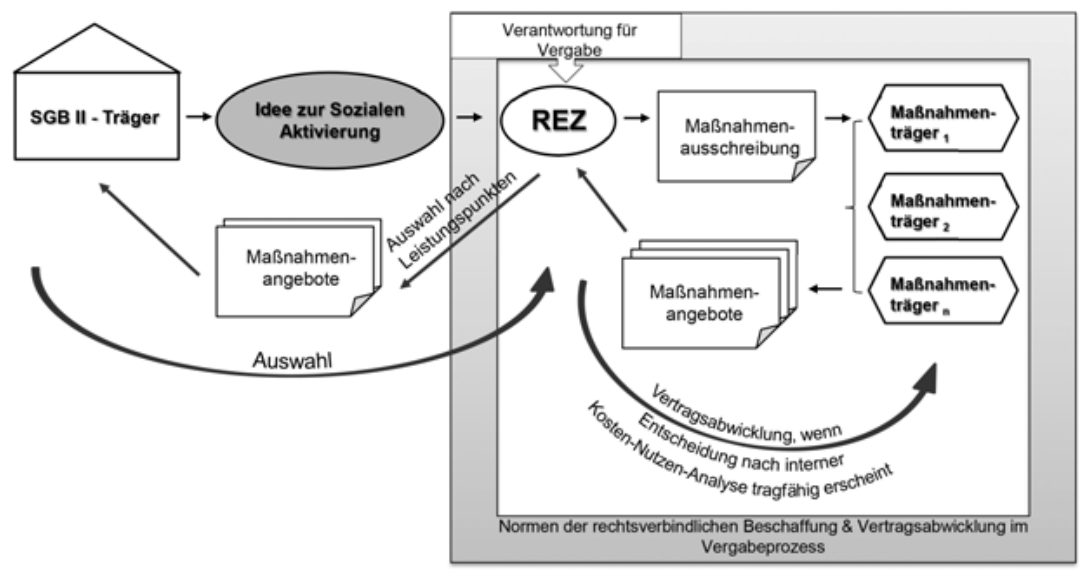

Quelle: Eigene Darstellung

1.) Das Jobcenter beauftragt das zuständige Regionale Einkaufszentrum mit der Maßnahmenausschreibung und gibt dabei Inhalte, Starttermin, geplante Teilnahmezahl sowie Personalbedarf an. Die Ausschreibung über das zuständige REZ wählen die befragten Fachkräfte des SGB-II-Trägers, insbesondere wenn es sich um arbeitsmarktnahe Standardprodukte ${ }^{29}$ handelt oder wenn die internen rechtlichen Fachkenntnisse oder Kapazitäten nicht ausreichen, um die Maßnahmenausschreibung korrekt durchzuführen. ${ }^{30}$ Nach der Ausschreibung durch das REZ kann das Jobcenter die von Maßnahmenträgern eingereichten Angebote selbst prüfen. Die Vergabe wird jedoch in Abstimmung mit dem REZ und damit im Einklang mit den der Bundesagentur für Arbeit inhärenten Vorgaben zur Maßnahmenfinanzierung vergeben, wie etwa die Ausrichtung an hohen Leistungspunkten verbunden mit Wirtschaftlichkeit. Denn wenn nach interner Kosten-Nutzen-Analyse der vom SGB-II-Träger gewünschte Maßnahmenträger ausscheidet, kann diesem nicht der Zuschlag gegeben werden, was durch das zu-

29 Standardprodukte sind in einem Maßnahmenkatalog der REZ gelistet, aus dem Agenturen und Jobcenter gängige Maßnahmen auswählen können.

30 Die Einführung von Arbeitsgelegenheiten und Antragsmaßnahmen wie dem Eingliederungszuschuss werden hingegen meist in Eigenregie der Jobcenter vorgenommen. 
ständige REZ geprüft wird. Entsprechend trägt das REZ die Verantwortung für die rechtsverbindliche Beschaffung und Vertragsabwicklung.

2.) Es kann jedoch auch der gesamte Beschaffungsprozess vom REZ vorgenommen werden, also die Ausschreibung, Auswahl und Beauftragung.

Abbildung 7: Maßnahmeneinkauf über das zuständige REZ

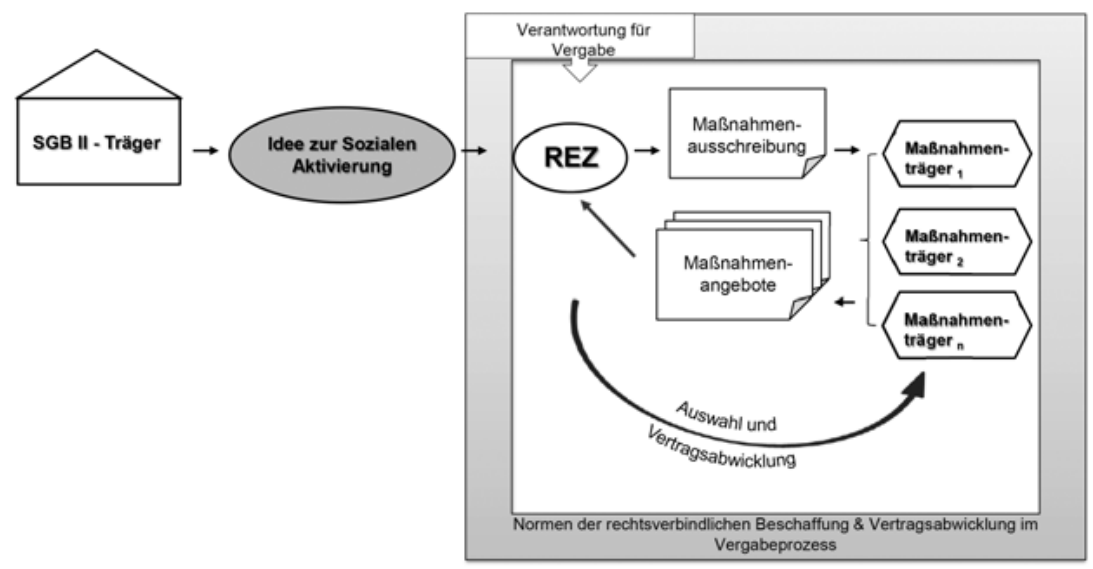

Quelle: Eigene Darstellung

Im Gegensatz zum oben gezeigten Modell (Abbildung 6), tritt der SGB-II-Träger erst nach der Vertragsabwicklung in Kontakt mit dem Maßnahmenträger. Diese Option wird insbesondere gewählt, wenn Standardprodukte eingekauft werden. Der Beschaffungsprozess von Maßnahmen bewegt sich nicht nur in einem rechtlichen, sondern auch in einem komplexen institutionellen Reglement. Aufgaben im Beschaffungsprozess sind seitens der SGB-II-Träger und der REZ äußerst kleinschrittig von der Information und Einschaltung der REZ bis zur Vertragsabwicklung festgelegt (BA 2010a). Die Abwicklung der Maßnahmenbeschaffung für die SGB-II-Träger muss das lokale REZ gegebenenfalls mit dem Fachbereich der BA-Zentrale abstimmen und auch die Vorstellungen der Regierung berücksichtigen:

»Wobei wir da natürlich auch ein bisschen dem politischen Druck folgen müssen. [...] wir brauchen eigentlich gar nicht so viele Arbeitsgelegenheiten, aber es ist gewollt, [...] dann müssen wir an diesem Programm noch ein bisschen herumbasteln.« (Markt- und Integrationsexpertin, Interview03Regio12)

Darüber hinaus würden Rückmeldungen von Jobcenter-Fachkräften berücksichtigt zum Beispiel mit bisher durchgeführten Trainingsmaßnahmen, um auch neue 
Ideen aufnehmen. Hier können die einzelnen Jobcenter neuartige Maßnahmen in Listen angeben. »So und darüber kommen in der Regel die Ideen.« (Markt- und Integrationsexpertin, Interview03Regio12) Dieses Verfahren wurde im Sample jedoch seitens der SGB-II-Träger-Fachkräfte nicht genannt. Doch auch die Abteilung der Markt- und Integrationsexpertin wird von ihrer Leiterin als Maßnahmen-innovatorisch dargestellt, beispielsweise wenn es gilt besondere Weisungen der Jobcenterverbund-Geschäftsführung umzusetzen. Hier trägt die Expertin ihre Ziele auch direkt einzelnen Maßnahmenträgern an.

Zusammenfassend gibt es bei dem Typ der Maßnahmenentstehung über das REZ die Option, 1.) den Einkaufsprozess größtenteils an das REZ zu übergeben, jedoch die Angebotsauswahl anhand der Kosten-Nutzen-Analyse bei den SGB-IITräger zu belassen oder 2.) den gesamten Einkaufsprozess inklusive der Vergabeentscheidung an das REZ auszulagern. SGB-II-Träger nutzen den Maßnahmeneinkauf über das REZ insbesondere dann, wenn es sich um konventionelle Maßnahmen handelt oder die internen Kenntnisse über die ordnungsgemäße Vergabe nicht genügen. Politische Vorgaben des BMAS, die eine Schwerpunktsetzung bei der Verausgabung der Mittel für die Beschäftigungsförderung setzen, prägen das Vergabeverfahren durch das REZ tendenziell stärker. Grundsätzlich sind jedoch auch Innovationen in der Beschäftigungsförderung durch das REZ ausdrücklich erwünscht. So werden potentielle Wege der Einführung neuartiger Instrumente eruiert und versucht, auf die Bedarfe der lokalen SGB-IITräger einzugehen. Im Zuge der Einführung von innovativen Elementen kann es auch zu einer verstärkten Kommunikation und Beratung zwischen dem REZ und einzelnen Maßnahmenträgern kommen, um konkrete Maßnahmenkonzepte verfahrensgemäß umsetzen zu können. Darin nähert sich das REZ einer eher lokal üblichen Maßnahmengenese an.

Insgesamt wurden im Kapitel 5.2.2 die unterschiedlichen Kontexte von Maßnahmen- und SGB-II-Trägern als Initiatoren von innovativen Maßnahmen aufgezeigt, wobei besonders die Kommunikationsoptionen vor der Maßnahmenplanung sowie während des -verlaufs eine passgenaue Förderung von arbeitsmarktferner und unbekannter Klientel positiv beeinflussen. Wenn die Arbeitsmarktdienstleistungen unter Mitwirkung der zuständigen REZ eingekauft werden, kann dies einerseits eine überaus verfahrensgetreue Abwicklung garantieren, jedoch andererseits auch die Implementierung von innovativen Maßnahmen, die auf eine arbeitsmarktferne Klientel zielen, beeinträchtigen. 


\subsubsection{Rechtliche Realisierungsform und lokale Umsetzungen der Maßnahmen Sozialer Aktivierung}

Die Maßnahmen Sozialer Aktivierung können der Intention einer kurzfristigen Arbeitsmarktintegration kaum gerecht werden und dennoch entstehen sie, eingebettet in das SGB II und versehen mit der Maßgabe die Hilfebedürftigkeit zu beenden oder zu verringern. So werden Maßnahmen der Sozialen Aktivierung von den AkteurInnen im Feld meist als Vorstufe, Zwischenschritt oder flankierendes Modul zur Arbeitsmarktintegration und Beendigung der (wirtschaftlichen) Hilfebedürftigkeit angesehen. Obwohl es anfänglich keinerlei formalisierte rechtliche Realisierungsformen für die Maßnahmen Sozialer Aktivierung gab, wurden sie in mehreren divergierenden Rechtsformen vorgefunden:

Tabelle 3: Rechtliche Realisierungsform von Maßnahmen Sozialer Aktivierung

\begin{tabular}{|l|l|}
\hline Maßnahme & Rechtsform \\
\hline Arbeitsgelegenheit & $\S 16$ d SGB II \\
\hline Freie Förderung & $\S 16$ f SGB II \\
\hline Vermittlungsbudget & $\S 45$ SGB III \\
\hline Bildungsgutschein & FbW-Maßnahme § 81 SGB III \\
\hline $\begin{array}{l}\text { Berufsvorbereitenden } \\
\text { Bildungsmaßnahmen } \\
\text { (BvB) }\end{array}$ & SGB III (§ 61) \\
\hline Aktivcenter & $\begin{array}{l}\text { §16 Abs. 1 SGB II i.V.m. § 45 SGB III (vor dem } \\
\text { SGB III) }\end{array}$ \\
\hline ESF & EU-Verordnungen \\
\hline $50+-M a ß n a h m e$ & $\begin{array}{l}\text { Perspektive 50plus - Beschäftigungspakte für Ältere } \\
\text { in den Regionen (Bundesprogramm) }\end{array}$ \\
\hline
\end{tabular}




\begin{tabular}{|l|l|}
\hline $\begin{array}{l}\text { Mischfinanzierung: } \\
\text { Mikroprojekte }\end{array}$ & $\begin{array}{l}\text { ESF-Förderung im Rahmen des Programms: »STÄR- } \\
\text { KEN vor Ort« nach EU-Verordnungen; Öffentliche } \\
\text { Körperschaften (z.B. Kommunen) tragen 15 Prozent } \\
\text { der Finanzierung, 85 Prozent werden über ESF-Mittel } \\
\text { und BMFSJ-Mittel durchgeführt }\end{array}$ \\
\hline Kommunale Mittel & \\
\hline Kirchliche Mittel & \\
\hline
\end{tabular}

Quelle: Eigene Darstellung

Da es sich bei Arbeitsgelegenheiten und Aktivcenter um die beiden zentralen rechtlichen Realisierungsformen der Maßnahmen Sozialer Aktivierung handelt, werden sie im Folgenden dargestellt.

\subsubsection{Arbeitsgelegenheiten (AGH)}

In dieser Studie war die Realisierungsform von Maßnahmen Sozialer Aktivierung als Arbeitsgelegenheit in der Mehraufwandsvariante nach § 16d Satz2 SGB II (AGH MAE, auch bekannt als »Ein-Euro-Job«, »Aktivjob« oder »Zusatzjob«) eine der beiden häufigsten. ${ }^{31}$ Diese Maßnahmen zielen auf arbeitsmarktferne erwerbsfähige Leistungsempfangende im Rechtskreis des SGB II sowie Jugendliche, die nach dem Eintritt in den Leistungsbezug kurzfristig keine Stelle gefunden haben. AGH MAE gelten als »ultima ratio« (BA 2011: 5), sollen also eingesetzt werden, wenn mit keinem anderen Instrument eine Integration in den ersten Arbeitsmarkt erwartet wird. Arbeitsgelegenheiten sind hinsichtlich

31 Zum Beispiel eine reine sportliche Betätigung in einem Verein in Verbindung mit AGH (Interview01Regio04); AGH für U25-Jährige Spiel- und Mediensüchtige (Interview02Regio04); AGH für drogen-und alkoholsüchtige Jugendliche (Interview02Regio04); AGH für trockene Alkoholiker (Interview02Regio05); Musikmaßnahme (Interview04Regio04); AGH für lebenspraktische Probleme (Interview02Regio05); Hundemaßnahme (Interview04Regio04), Gesundheitsbezogene Maßnahme (Interview02Regio05); diverse AGH im Sozialbetrieb (Interview05Regio06); diverse AGH im Familienbezogenen Maßnahmenträger; Praktische Lebenshilfe für Alleinerziehende (Interview02Regio11), Jugend-AGH (Interview01Regio12); Musisch-Sportliche AGH (Interview04Regio12) Tanzen, Gymnastik, Klettern, Kampfkunst, MusikWorkshops, Theater in AGH; AGH mit Strafgefangenen (Interview04Regio12); Theater-AGH (Interview02Regio13); Medienbezogene AGH (Interview02Regio13). 
der Zuweisung von arbeitsmarktfernen respektive -nahen Teilnehmenden, der Wirkung auf die Beschäftigungsfähigkeit sowie der Eintrittschancen in den Arbeitsmarkt breit erforscht (zum Beispiel Dengler 2013, Sowa/Klemm/Freier 2012, Achatz/Fehr/Schels/Wolff 2012, Freier 2011, Hohendanner/Klemm/Promberger/Sowa 2010, Hohmeyer/Wolff 2010). ${ }^{32}$ Für diese Studie sind AGH von besonderer Bedeutung, da Maßnahmen Sozialer Aktivierung primär über diese Rechtsform eingeführt und tendenziell anschließend in Aktivcentern ${ }^{33}$ weitergeführt wurden. Zwar ist die Verteilung der Realisierungsformen von Maßnahmen Sozialer Aktivierung in dieser qualitativ angelegten Studie nicht repräsentativ, doch wird die hier vorgefundene Dominanz der Arbeitsgelegenheiten durch das Faktum unterstützt, dass Arbeitsgelegenheiten, insbesondere in der Mehraufwandsvariante, bundesweit eines der am intensivsten genutzten Instrumente der Leistungen zur Eingliederung sind (BA 2011: 3, BA 2013e: 41). Arbeitsgelegenheiten können dazu dienen, Maßnahmen ohne eine Ausschreibung zu realisieren und mit dem Maßnahmenträger >des Vertrauens` kooperieren zu können (zum Beispiel Regio05, Interview04Regio06). AGH können als Ausschreibungsfreie Projektförderung gestaltet werden (Interview04Regio06).

Arbeitsgelegenheiten haben schon seit ihrer Einführung ein breites Spektrum an Einsatzbereichen und Wirkmöglichkeiten (Sowa et al. 2012). Die Offenheit des Instruments bietet auch der Einführung von Innovationen eine hinreichende Grundlage, wie auch diese Erhebung zeigt. Zudem ist der Ansatz, erste, meist einfache Tätigkeiten wie zum Beispiel Fahrradreparaturen, Ausbessern von Kleidungsstücken und Kochen für den Eigenbedarf in der Maßnahme zu verankern ein motivierendes Element für Klientel-Gruppen, die für die staatlichen Institutionen nicht einfach zu erreichen sind (wie zum Beispiel Wohnungslose).

Zusammengefasst entstehen Maßnahmen Sozialer Aktivierung häufig in Arbeitsgelegenheiten, die als flexibles Instrument gelten und dadurch innovative Elemente ermöglichen. Hier können Maßnahmen für arbeitsmarktferne Zielgruppen konzipiert, getestet und weiterentwickelt werden.

32 Regionalspezifische Studien zu Arbeitsgelegenheiten gibt es beispielsweise für München (Koch/Fertig 2012) und zu Hamburg (Helmut/Fertig/Koch/Osiander 2011).

33 Vergleiche Kapitel 5.3 Das Aktivcenter: Professionalisierung und Zeichen einer Bottom-up-Entwicklung. 


\subsubsection{Aktivcenter nach § 16 Abs. 1 SGB II i.V.m. § 45 SGB III}

Das Aktivcenter nach $\S 16$ Abs. 1 SGB II i.V.m. $\S 45$ SGB III $^{34}$ zeichnet sich durch sogenannte »Schlüsselqualifikationen« und »Gesundheitsorientierung« als zentrale Bestandteile sowie eine bemerkenswerte zeitliche Flexibilität aus: eine Maßnahme dieser Rechtsform kann als Teilzeitmaßnahme zugewiesen werden und ermöglicht auch unterweisungsfreie Zeiten. ${ }^{35}$ Das Aktivcenter wurde im September 2010 von der zentralen Maßnahmenentwicklung der Bundesagentur für Arbeit im § 16 I SGB II i.V.m. § 46 I S. 1 SGB III geschaffen. ${ }^{36}$ Genutzt wurde es häufig als Vehikel zur Einführung von Maßnahmen Sozialer Aktivierung. Zuvor wurden ähnliche Maßnahmeninhalte sozialer Aktivierung in einem anderen Maßnahmentyp (meist als Arbeitsgelegenheit in der Mehraufwandsvariante) eingeführt und als Maßnahmenkonzept in etwas gewandelter Form mit der Instrumenteneinführung des Aktivcenters weitergeführt. Aktivcenter-Maßnahmen kümmerten sich um die Teilnehmenden mehr »in der Gesamtheit« (Marktund Integrationsexpertin, Interview03Regio12). Zudem sei es ein sehr niederschwelliges Angebot vorrangig für über 25-jährige erwerbsfähigen Hilfebedürftigen des SGB-II-Regelkreises

»mit ausgeprägtem Unterstützungsbedarf, die

1.) auf andere Weise nicht erreicht werden können, um sie für eine berufliche Qualifizierung oder eine Beschäftigungsaufnahme zu motivieren und schrittweise heranzuführen und

2.) wegen der in ihrer Person liegenden Gründe ohne diese Förderung nicht bzw. noch nicht eingegliedert werden können.« (BA Regionaldirektion Regionales Einkaufszentrum 2010: 11)

Unter dem Aspekt Gesundheitsorientierung werden Stressbewältigung, Bewegung, gesunde Ernährung und der Umgang mit Suchtproblematiken realisiert. Die Maßnahmenteilnehmenden sollen sensibilisiert und motiviert werden »für eine gesundheitsbewusste Lebensführung, die ihre Eingliederungsfähigkeit er-

34 Vor dem 01.04.2012 war das Aktivcenter verankert in § 16 I SGB II i.V.m. § 46 I S. 1 SGB III.

35 Es besteht ein Anspruch auf zwei unterweisungsfreie Arbeitstage für jeden vollen Kalendermonat der Teilnahme.

36 Das Aktivcenter wird 2012 in seinen Grundsätzen in die rechtliche Fassung des $\S 45$ SGB III überführt und bietet seitdem zusätzlich die Option, einen Vermittlungsgutschein für die Maßnahme auszustellen (Kassel 2010). 
höht« (BA Regionaldirektion Regionales Einkaufszentrum 2010: 23). Die Stärkung des Selbstbewusstseins und auch im Bereich persönlicher und sozialer Kompetenzen wurden Elemente der sozialen Aktivierung verankert.

So werden beispielsweise ein Tanzkurs (Interview03Regio06), Sportelemente für Jugendliche (Interview01Regio02) und eine Maßnahme für Wohnungslose (in Regio06 ${ }^{37}$ ) im Rahmen eines Aktivcenters etabliert. In einem Jobcenter in Regio11 wird das Aktivcenter dagegen primär genutzt, um die Verfügbarkeit für den Arbeitsmarkt zu prüfen: Die niederschwellige Maßnahme bietet Bewerbungstraining, ein Modul zur Gesundheitsorientierung und gewährleistet bei Bedarf eine Kinderbetreuung. Für die Teamleiterin ist jedoch die Anwesenheitspflicht von zentraler Bedeutung, denn diese soll dazu dienen, Personen mit häufigen Krankschreibungen zu erreichen (Interview03Regio11). Die anwesenheitspflichtige Maßnahme besitzt einen sechsmonatigen Zuweisungskorridor in dem die Teilnehmenden ab einem flexiblen Startzeitpunkt an einer Maßnahme teilnehmen müssen. Wer eine Krankschreibung einreicht, scheidet nicht aus, sondern tritt die Maßnahme zum Zeitpunkt seiner Genesung erneut an.

Insgesamt wurden 2012 durchschnittlich 473.000 Personen mit arbeitsmarktpolitischen Instrumenten in Kostenträgerschaft des SGB II gefördert (BA 2013e: 17). In Aktivcentern wurden 2012 dagegen 16.659 Personen zugewiesen. Im Vergleich zu dem Gesamtangebot der Maßnahmen zur Aktivierung und beruflichen Eingliederung nehmen Maßnahmen Sozialer Aktivierung, wie die folgende Tabelle 4 zeigt, quantitativ betrachtet, einen sehr geringen Stellenwert ein. Hier bestätigt sich erneut, dass es sich um eine sehr spezifische Klientel handelt.

Tabelle 4: Teilnahmen in Maßnahmen zur Aktivierung und beruflichen Eingliederung (MAbE)

\begin{tabular}{|l|c|c|c|c|c|}
\hline Maßnahme & \multicolumn{1}{c|}{$\mathbf{2 0 1 0}$} & $\mathbf{2 0 1 1}$ & \multicolumn{1}{c|}{$\mathbf{2 0 1 2}$} & \multicolumn{1}{c|}{$\mathbf{2 0 1 3}$} & \multicolumn{1}{c|}{$\mathbf{2 0 1 4}$} \\
\hline MAbE gesamt & 1.620 .652 & 1.201 .251 & 1.112 .588 & 1.184 .903 & 1.246 .081 \\
\hline Aktivcenter SGB II & 26.410 & 18.450 & 16.659 & 14.036 & 13.202 \\
\hline
\end{tabular}

Quelle: Statistische Auswertung der Bundesagentur für Arbeit (BA 2016)

Darüber hinaus wurde das Aktivcenter in seinem Entstehungsjahr 2010 über 26.000 Mal mit Teilnehmenden besetzt, 2014 dagegen nur noch mit über 13.000.

37 Vergleiche Kapitel 5.1.5 Fallbeispiel einer Maßnahme für Wohnungslose. 
Die interviewten SGB-II-Träger-Fachkräfte schrieben eine abnehmende Nutzungstendenz dabei insbesondere immer knapperer Finanzressourcen zu. Diese Aussagen lassen sich bis zu einem gewissen Grad durch den deutlichen Rückgang der Maßnahmenzuweisungen insgesamt bestätigen. Von 2010 auf 2011 ging die Zahl nahezu um ein Viertel zurück. Doch die Maßnahmenpraxis ist sehr vielfältig und auch die Zielvorstellungen. Da das Aktivcenter eine Besonderheit in der hierarchisch und zentral gesteuerten Bundesagentur für Arbeit darstellt, wird dessen Entstehung im Kapitel 5.3 genauer betrachtet.

Zusammengefasst wurde in Kapitel 5.2.3 deutlich, dass für die Erreichbarkeit einer spezifischen Klientel äußerst niederschwellige und zum Teil auch ansprechende Instrumente als notwendig erachtet werden. Für diese soziale Aktivierung wurden (rechtlich-finanzielle) Maßnahmeninstrumente gefunden, die innovative Elemente zulassen. Dabei bieten diverse Maßnahmeninstrumente unterschiedliche Freiräume und Entstehungskontexte für Maßnahmen Sozialer Aktivierung. Die untersuchten Maßnahmen entstanden meist als Arbeitsgelegenheit in der Mehraufwandsvariante, einem bundesweit häufig und vielfältig genutzten Beschäftigungsförderungsmittel. Eine Fortführung wurde meist im neu geschaffenen Aktivcenter vorgenommen. ESF-Mittel und Kofinanzierungen können darüber hinaus Aspekte für niederschwellige Maßnahmen beitragen, jedoch in begrenztem Umfang. Der Kontext der rechtlichen und politischen Reglementierung stimuliert die Nutzung sozialer Aktivierung, wenn diese niederschwelligen Maßnahmen als Standardinstrument eingekauft werden können.

\subsection{Das Aktivcenter: Professionalisierung und ZEICHEN EINER BOTTOM-UP-ENTWICKLUNG}

Die Praktiken des Einkaufs niederschwelliger Maßnahmen bewirkten eine Dynamik: Es lässt sich eine Bottom-up-Entwicklung beobachten, da zentrale Inhalte sozialer Aktivierung aus dem Praxisfeld in die überregionale Maßnahmengestaltung Eingang gefunden haben: In Reaktion auf fortgesetzte Anfragen der SGB-II-Träger-Fachkräfte wurde seit September $2010^{38}$ ein bundesweit einheitliches Maßnahmenangebot geschaffen, das dezidiert Maßnahmen mit niederschwelligen Anforderungen beziehungsweise für eine arbeitsmarktferne Klientel

38 Zunächst verankert als Aktivcenter im § 16 I SGB II i.V.m. § 46 I S. 1 SGB III und ab dem 01.04.2013 im $§ 16$ Abs. 1 SGB II i.V.m. § 45 SGB III. 
auflegt und als zentrale Bausteine sogenannte Schlüsselqualifikationen und Gesundheitsorientierung ${ }^{39}$ beinhaltet.

»Wir haben erkannt, vor einigen Jahren, dass wir niederschwellig anfangen müssen, also dass, das es Kunden gibt, die ja, einfach, dieses gewaschen kommen, einfach dieses halbwegs ordentlich kommen und ich sag mal, wenn ich das schon nicht schaff zu einer Behörde zu gehen, wie soll ich das dann schaffen, wenn ich zum Arbeitgeber gehe?« (Teamleiterin, Interview04Regio02)

Doch die Einführung dieser niederschwelligen Maßnahmen gestaltete sich zunächst als »schwierig, sehr, sehr schwierig« (Teamleiterin, Interview04Regio02), da solcherlei Maßnahmen nicht bekannt waren bei den REZ. »Die waren auf ihrer SGB-III-Schiene und jetzt kamen dann ARGEn daher mit, ja wir müssen da was machen mit die Leute in der Früh abholen und solche Sachen und das war ganz schwer.« (Teamleiterin, Interview04Regio02) Aus Sicht der AkteurInnen vollzog sich ein langwieriger Entstehungsprozess, bis die niederschwelligen Maßnahmenformen im wechselseitigen Austausch der verschiedenen vertikalen Ebenen so gestaltet wurden, dass sie auf Akzeptanz stießen, denn

»wir [das Fallmanagement; CF] haben also eine sehr lange Zeit gebraucht, um diese Maßnahme der sozialen Aktivierung zu realisieren. Wir haben immer wieder mit dem REZ zusammengesessen und Modelle entwickelt und erst mit der Maßnahme [...], erst da haben wir einen Ansatz gefunden unsere Ideen in Ansätzen umzusetzen.« (Fallmanager, Interview01Regio10)

Der Versuch, niederschwellige Maßnahmen standardisiert einzurichten wurde erst über die Modernisierung der Arbeitsmarktinstrumente ermöglicht. Seitdem das Aktivcenter im Rahmen des $§ 46$ SGB III geschaffen wurde haben diese Instrumente einen festen Platz im Portfolio. Sowohl aus Interviews mit SGB-IITrägern, wie auch aus einem Interview mit einem lokalen REZ sowie einer Mitarbeiterin der fachlichen Steuerung der REZ geht hervor, dass die Nachfrage mehrerer Arbeitsgemeinschaften (etwa in Regio06, Regio01 oder Regio02) und der zunehmende Aktivierungsdruck einer weit vom Arbeitsmarkt entfernten Kli-

39 Die frühere Bezeichnung »Gesundheitsförderung« wurde in der BA durch »Gesundheitsorientierung« ersetzt, da Gesundheitsförderung ein feststehender Begriff im SGB V ist und in Verbindung gebracht wird mit dem § 20 SGB V zur Prävention sowie Selbsthilfe und dem Aufgabenbereich der Krankenkassen (Interview07Regio02). 
entel eine Ausweitung des Maßnahmenportfolios befördert haben. Die Produktbetreuerin schildert die Schaffung des Aktivcenters nach $\S 16$ I SGB II i.V.m. $\S 46$ I S.1 SGB III als Reaktion auf Maßnahmennachfragen von SGB-II-Trägern für Personen mit gesundheitlichen und sozialen Einschränkungen:

»Wir [VertreterInnen eines lokalen REZ; CF] brauchen was für Leute die weit weg sind, wir brauchen was für Leute, die nicht den ganzen Tag anwesend sein können, wir brauchen was für Leute, die gesundheitliche Einschränkungen haben, wir brauchen was für Leute die sich in der Arbeitslosigkeit eingemuggelt haben. Wir brauchen irgendwas, das interessant ist, damit die Mal was Anderes machen und nicht zu Hause auf der Couch liegen. Das waren die Grundansätze.« (Produktbetreuerin, Interview06Regio02)

So ist auch das Aktivcenter gemäß der Vergabeunterlagen vorrangig darauf ausgerichtet, über 25-jährige, erwerbsfähige Hilfebedürftige »mit ausgeprägtem Unterstützungsbedarf, die

»auf andere Weise nicht erreicht werden können, um sie für eine berufliche Qualifizierung oder eine Beschäftigungsaufnahme zu motivieren und schrittweise heranzuführen und wegen der in ihrer Person liegenden Gründe ohne diese Förderung nicht bzw. noch nicht eingegliedert werden können.« (BA Regionaldirektion Regionales Einkaufszentrum 2010: 11)

Dieses »noch nicht« thematisiert auch die befragte Produktbetreuerin: »Das war auch der Ansatz bei dem Grundgedanken, dass Aktivcenter zu kreieren, zu gestalten, das wir Leute haben, die sehr weit weg sind und das wir die erst Mal aktivieren und an den Arbeitsmarkt heranführen müssen.« (Produktbetreuerin, Interview06Regio02)

Das Aktivcenter wird als bundesweites Standardprodukt durch die jeweiligen lokalen REZ angeboten, kann jedoch auch individualisiert oder in Eigenregie des SGB-II-Trägers eingekauft werden. Abwechslungsreich sollten die Maßnahmen sein, aber auch für den Anfang nur mit drei (sukzessive bis zu acht) täglichen Stunden Anwesenheitspflicht, denn dies ist eine Definition der Erwerbsfähigkeit und müsste daher auch von weit vom Arbeitsmarkt entfernten Personen geleistet werden können. An dieser Stundenzahl zeigt sich besonders deutlich die Orientierung an den Minimalanforderungen. ${ }^{40}$ Das Aktivcenter wurde so ein offenes

40 Diese Anregungen kommen auch aus den persönlichen Gesprächen der Produktbetreuerin mit SGB II-Leistungsempfangenden (Interview06Regio02). 
Standardmaßnahmenprodukt, als Bindeglied zwischen dem Fernziel Arbeitsmarkt und der Betreuung einer sehr arbeitsmarktfernen Klientel: »Vom Gesetzgeber her ist der 46 unglaublich weit ausgerichtet, na also unter Aktivierung, Heranführung an den Arbeitsmarkt kann ich alles fassen.« (Produktbetreuerin, Interview06Regio02)

Der Blick auf die spezifischen Fähigkeiten und angenommenen Bedürfnisse Langzeitarbeitsloser und ein passgenaues Maßnahmenportfolio wird von einer Gesundheitsexpertin in der Bundesagentur für Arbeit als Erleichterung und Effektivierung der Arbeit des Fallmanagements betrachtet:

»Es werden laufend neue Zahlen veröffentlicht, dass die psychische Belastbarkeit doch immer schlechter wird, unabhängig jetzt davon ob mit Beschäftigung oder ohne Beschäftigung. Wir gucken uns ja nur die Arbeitslosen an und das ist auch etwas, was vor allem die Fallmanager, die ich ja auch qualifiziere, sehr beschäftigt. Der Umgang mit diesen Kunden ist nicht einfach und eine Integration ist oftmals noch schwieriger beziehungsweise kommt es häufig zu diesem Drehtüreffekt, kurzfristige Arbeitsaufnahme, dann erneute Arbeitslosmeldung.« (Expertin für Gesundheitsorientierung, Interview07Regio02)

Gesundheitsorientierung kommt in der Maßnahmengestaltung des SGB-IIRechtskreises erst in jüngerer Zeit Bedeutung zu, insbesondere mit der Schaffung des Aktivcenters. Dem ging ein weiterer verwaltungsinterner Entwicklungsprozess voraus: Durch ein Sachverständigengutachten aus dem Jahr 2007 wurde die Gesundheit prominentes Thema. Denn das Gutachten beobachtete einen Zusammenhang von Arbeitslosigkeit und Krankheit: Gesundheitliche Einschränkungen begünstigen Arbeitslosigkeit, führen zu einer weniger wirksamen Arbeitsvermittlung und die Arbeitslosigkeit selbst beeinträchtige den gesundheitlichen Zustand noch weiter. Die Berichtergebnisse wurden innerhalb des BMAS diskutiert. Darüber hinaus wurde die Beschaffenheit der Gesundheits- beziehungsweise Einschränkungsstruktur der SGB-II-Bezieher eruiert, um Aufschluss über angemessene Maßnahmeninhalte zu erlangen. Schließlich fanden Gesundheitsorientierung Stressbewältigung, Bewegung, gesunde Ernährung und der Umgang mit Suchtproblematiken Eingang in das Maßnahmenangebot, um Maßnahmenteilnehmende zu sensibilisieren und zu motivieren »für eine gesundheitsbewusste Lebensführung, die ihre Eingliederungsfähigkeit erhöht« (BA Regionales Einkaufszentrum 2010: 23). Daneben werden persönliche und soziale Kompetenzen als Elemente sozialer Aktivierung gesetzlich verankert: 
»Insbesondere sollen gefördert werden:

- Persönliche Kompetenzen (z.B. Motivation, Leistungsfähigkeit, aber auch Selbstbild, Selbsteinschätzung)

- Soziale Kompetenzen (z.B. Kommunikation, Kooperation/Teamfähigkeit, Konfliktfähigkeit, Aufbau von Tagesstrukturen über einen längeren Zeitraum)

- Methodische Kompetenzen (z.B. Problemlösung, Arbeitsorganisation, Lerntechniken, Einordnung und Bewertung von Wissen)« (BA Regionaldirektion Regionales Einkaufszentrum 2010: 21)

Mit der Neuausrichtung im Januar 2009 wurden Trainingsmaßnahmen und die Beauftragung Dritter mit der Vermittlung ( $\$ 37$ SGB III) in einem Paragraphen zusammengefasst dem $\S 46$ SGB III. ${ }^{41}$ Die Produkte für Personen im Rechtskreis SGB II werden seit 2010 entwickelt und ausgebaut, so dass auch hier die Gesundheitsorientierung aufgenommen wird, anhand der 20 Prozent einer Maßnahme der Verbesserung der Gesundheit dienen können (Produktbetreuerin, Interview06Regio02). Die in der Maßnahme erbrachten Leistungen dürfen weder die Krankenkassenleistungen zur primären Prävention nach $\S 20$ SGB V noch die kommunalen Eingliederungsleistungen nach § 16a SGB II (psychosoziale Betreuung sowie Suchberatung) ersetzen und die Maßnahmenträger sollen lokal mit Krankenkassen und Kommunen kooperieren, um dies zu gewährleisten. Trotz dieser komplexen Abgrenzungsbedürfnisse ist die Gesundheitsorientierung als regelmäßiges Angebot über die gesamte Laufzeit vorzuhalten.

So beinhaltet die Beantragung von sozial aktivierenden Elementen immer die Klärung von Zuständigkeiten für gewünschte Leistungen, die häufig auch Interpretations- und Auslegungsprozesse beinhalten. Angestellte des REZ in Regio02 verfahren hier im Zeitverlauf unterschiedlich:

»Vor drei, vier Jahren konnten wir keine sozialpädagogische Betreuung in einer Maßnahme kaufen, das das das gab es einfach nicht und dann gab es so viele ARGEn, die das immer wieder nachgefragt haben und auch so viele ich denke auch Agenturen, die das nachgefragt haben, bis das sich dann geöffnet hat und also alle sich bewegt haben und jetzt kann man's kaufen. Ja, also das kommt bei uns eigentlich alles von unten nach oben, diese ganze Planung, diese ganzen Ideen [...] Es gibt einfach auch viele Fallmanager oder Vermittler mit verschiedener Vorerfahrung, also wir haben Leute bei uns beschäftigt, die wa-

41 Seit dem 01.04.2012 im $\S 45$ SGB III. 
ren früher in der Jugendarbeit oder die waren früher in der Drogenhilfe tätig und deren Erfahrungen und Ideen, die muss man doch nutzen.« (Teamleiterin, Interview04Regio02)

Durch die Einführung des Aktivcenters ist es auch Beschäftigten des REZ möglich, niederschwellige Maßnahmenpakete zeitnah einzukaufen. Diese Veränderung wird auf Ebene der SGB-II-Träger teilweise wohlwollend registriert und genutzt:

»Wenn wir jetzt heutzutage mit so etwas daher kommen, dann rollen sich bei denen nicht mehr die Fingernägel nach oben, sondern dann sagen die ja, schauen wir wie wir das hinkriegen [...], weil dieser Paragraph 46 hat ja auch viele Facetten, die man da mitbenutzen kann, wo kriegen wir denn rein, was ihr da haben wollt« (Teamleiterin, Interview04Regio02)

Besonderheit des Aktivcenters ist, dass hier keine Eingliederungsquoten von den Regionalen Einkaufszentren oder den SGB-II-Trägern vorgegeben werden. Dieser Umstand mache die Qualitätssicherung schwieriger, so die Produktbetreuerin (Interview06Regio02). Doch die Unabhängigkeit von Integrationsquoten kann auch zu einer sozialen Aktivierung führen, deren Integrationserfolg vielleicht nicht in einer Anstellung in den ersten Arbeitsmarkt zu messen ist.

Zusammenfassend haben im Instrument des Aktivcenters Schlüsselqualifikationen und Gesundheitsorientierung einen Anteil in einem bundesweit einsetzbaren Standardinstrument der Beschäftigungsförderung gefunden. Diese Aspekte wurden in den hier konzeptionell erarbeiteten Maßnahmen Sozialer Aktivierung gefasst. Eine herausragende Rolle nimmt die Einführung des Aktivcenters ein, da es flankiert durch einen Prozess der wissenschaftlichen Begleituntersuchung der Gesundheit von Langzeitarbeitslosen insbesondere durch die intensive Nachfrage von SGB-II-Trägern erklärbar ist. Die Steuerung der BA und die politischen Richtlinien des BMAS haben hier eine Entwicklung der niederschwelligen Maßnahmengestaltung getragen, in dessen Rahmen die Qualitätssicherung nicht allein über Eingliederungsquoten durchführbar ist. Die Einführung des Aktivcenters verweist auf eine von den SGB-II-Trägern wahrgenommene Notwendigkeit von niederschwelligen Maßnahmen, die Personen sozial aktiviert, mit dem Ziel, sie langfristig in Erwerbstätigkeit zu integrieren. 


\subsection{SPANNUNGSFELDER IN DER INSTALLIERUNG VON MASSNAHMEn SOZIALER AKTIVIERUNG}

Spannungsfelder entstehen zwischen dem Aktivierungsdiskurs und seiner Praxis innerhalb der Arbeitsverwaltung, wenn eine sehr arbeitsmarktferne Klientel von den AkteurInnen der Arbeitsverwaltung saktiviert marktdienstleistungen stehen in einem Rechtsrahmen, der Standardprodukte vorhält, aber auch modifizierte Standardprodukte und die Etablierung neuer Elemente unter bestimmten Kriterien zulässt. Die den Zielsetzungen des Aktivierungsdiskurses entsprechenden Standardprodukte zielen auf eine rasche Arbeitsvermittlung bei kurzzeitiger Maßnahmendauer. Am anderen Ende des Spektrums sind die Maßnahmen Sozialer Aktivierung einzuordnen. Im Folgenden werden Spannungsfelder aufgezeigt, die die Installierung von Maßnahmen Sozialer Aktivierung mitbedingen. In der Kommunikation mit den Maßnahmenträgern muss zwischen Aktivierung und Wettbewerbsprinzip abgewogen werden (Kapitel 5.4.1). In der Unterstützung durch die Regionalen Einkaufszentren muss die Garantie der Rechtskonformität versus Bürokratisierung ins Verhältnis gesetzt werden (Kapitel 5.4.2). Hinsichtlich der institutionellen Rahmenbedingungen stehen die SGB-II-Träger vor Begrenzungen durch die vermittlungsorientierte Budgetierung (Kapitel 5.4.3) sowie Implikationen der Eingliederungsquote (Kapitel 5.4.4). Insgesamt begünstigen diese Rahmenbedingungen die Vermittlung einer arbeitsmarktnahen Klientel. Insofern ist gerade der Anspruch der AkteurInnen der Arbeitsverwaltung erschwert, die »verfestigte Arbeitslosigkeit« zu bearbeiten (Kapitel 5.4.5). Die Spannungsfelder manifestieren sich in einer verstärkten Nachfrage an niederschwelligen Maßnahmen durch SGB-II-Träger-Fachkräfte, die die Entwicklung eines neuen Standardinstruments bottom-up mitbedingt (Kapitel 5.4.6).

\subsubsection{Abwägen zwischen Aktivierung und Wettbewerbsprinzip}

Von SGB-II- und Maßnahmenträgerbeschäftigten wird insbesondere das Ziel formuliert, eine arbeitsmarktferne und häufig schwer erreichbare Klientel zu aktivieren. In verschiedenen Bereichen kann es zu einem schmalen Weg zwischen Aktivierung und Rechtskonformität kommen, da sich die niederschwellige Angebotsstruktur für diese spezifische Klientel oftmals an Grenzen verschiedener Förderinstitutionen befindet, wie im Folgenden zu zeigen sein wird. 


\subsubsection{Autonomie im Entstehungsprozess der Maßnahme}

Die Gestaltbarkeit von Maßnahmen, insbesondere nach ihrem Beginn, um diese an die Anforderungen einer arbeitsmarktfernen Klientel anpassen zu können, benötigt in der Vorstellung der AkteurInnen ein hohes Maß an Autonomie der SGB-II-Träger- und teilweise auch der Maßnahmenträger-Fachkräfte. AGHs bieten mehr Gestaltungs- und Kommunikationsfreiräume zwischen SGB-II- und Maßnahmenträgern. Seitens der SGB-II-Träger kann eine freihändige Vergabe sehr viel mehr Autonomie bedeuten:

»Weil da können wir wenigstens den Träger aussuchen. Ja da haben wir mehr Mitspracherecht und mehr Entscheidungsrecht, als das wir uns über das Einkaufszentrum dafür auch bewerben und dann das Problem haben, dass wir schon beim Lesen der Konzepte wissen, dass es schief laufen wird.« (Teamleiterin, Interview02Regio05)

Anschließend folgt dann ein Verfahren der »kleinen Ausschreibung « (Teamleiterin, Interview02Regio05), indem die Leistungsbeschreibung an drei bis vier Träger gesandt und nach einem formlosen Austausch die Umsetzung des geeignetsten Konzeptes begonnen wird.

In der Zusammenschau zeigt sich, dass diese bereits im Vergabeverfahren relevanten Abstimmungs- und Kommunikationsmöglichkeiten zwischen SGB-IIund Maßnahmenträgern im Umsetzungsprozess eine entscheidende Rolle für die Entstehung niederschwelliger Maßnahmen besitzen. Dass Informationsveranstaltungen einberufen werden, weist auf spezifische Maßnahmenanforderungen und Unwägbarkeiten, ob der dann erscheinenden Klientel hin. Denn hier beginnt ein Entwicklungsprozess mit der Frage, wie neuartige, regional abgestimmte Maßnahmen gestaltet werden müssten. Dafür werden bestehende Erfahrungen der SGB-II- sowie der Maßnahmenträger herangezogen. Weiter zeigt sich, dass diese Maßnahmen häufig in Maßnahmenträgern durchgeführt werden, die den SGB-II-Trägern bereits als besonders positiv bekannt sind. Dagegen gehen Vorerfahrungen mit den Maßnahmenträgern nicht in die Bewertungsschemata der Vergabeverfahren des REZ ein. ${ }^{42}$ Zudem kann bei einer eigenständigen Vergabe durch den SGB-II-Träger über Absprachen auch nach der Ausschreibung noch Einfluss auf den Leistungskatalog genommen werden. Eine Möglichkeit, die die

42 So führt die Produktbetreuerin eines REZ aus, dass sich teilweise über Jahre hinweg eine vertrauensvolle Zusammenarbeit zwischen Maßnahmen- und SGB II-Trägern gebildet habe, was durch das zentrale Vergabeverfahren gehemmt werden könne (Interview06Regio02). 
REZ mit ihren juristisch standardisierten Verfahren nicht vorsehen können: »So und da kann sich der Bildungsträger dann auch auf seinen Vertrag zurückziehen und sagen, das steht hier ja nicht drin.« (Fallmanager, Interview01Regio10)

»Wir haben das früher [als Arbeitsberater in Zeiten seiner Arbeitsamtstätigkeit; CF] [...] beispielsweise so gehabt, wenn da ein Bildungsträger etwas machen wollte, haben wir den eingeladen und gesagt und dann bring deine Dozenten mit und dann befragen wir die und sagen, wie stellen sie sich das denn so vor und dann haben wir anschließend gesagt, da musst du dir einen neuen suchen, der passt nicht. Das was wir damit wollen ist mit dieser Denk- und Arbeitsstruktur nicht zu erreichen« (Fallmanager, Interview01Regio10).

Bei einem Einkauf über ein REZ ist der Zugriff auf die konkreten Maßnahmeninhalte und Regelungen erschwert, da hier im Vorfeld einer Ausschreibung nicht mit den Trägern kommuniziert werden kann (Interview02Regio05). Häufig beabsichtigen Maßnahmenträger und die SGB-II-Träger-Fachkräfte einen Austausch gerade auch während der Maßnahmendurchführung. Das Fallmanagement erhofft insbesondere zur Erreichung einer sehr arbeitsmarktfernen Klientel einen stärkeren Kontakt zu der Maßnahme und dem Maßnahmenträger, um einen besseren Zugang zu den Teilnehmenden zu erreichen. Einzelgespräche des Fallmanagements mit Maßnahmenteilnehmenden werden beispielsweise in Regio10 schon in der Ausschreibung verankert, um vor Ort im Maßnahmenträger mit den Maßnahmenteilnehmenden zu sprechen, zum Beispiel zu möglichen Qualifizierungsangeboten zu beraten und die Eingliederungsvereinbarung zu aktualisieren »und dann ist das ja eine ganz andere Gesprächsatmosphäre." (Fallmanager, Interview01Regio10) Denn das in der Maßnahme gewonnene Vertrauen führe auch zu einer größeren Offenheit gegenüber dem Fallmanagement. ${ }^{43}$ Daneben wird der Kontakt für einen Austausch zwischen dem Fallmanagement und den Maßnahmenträgerbeschäftigten genutzt und auftretende Fragen gleich zu Beginn behandelt (Interview01Regio10).

\subsubsection{Flexibilität im Maßnahmendesign für eine zum Teil unbekannte Klientel}

Wenn Jobcenter/Arbeitsgemeinschaften nicht die Dienstleistungen des REZ nutzen, kann dies unter anderem darin begründet sein, dass sich die SGB-II-Träger

43 Auch in einer Jugendmaßnahme gehen Maßnahmenträgerbeschäftigte mit den Teilnehmenden in das Jobcenter, um die Klientel für die Arbeitsvermittlung zugänglich zu machen und das aufgebaute Vertrauen zu nutzen (Interview02Regio12). 
Zeit und Ressourcen sparen möchten. Dann konkretisieren sie die Maßnahmenziele und -inhalte nicht zu Beginn, sondern treten zunächst mit Maßnahmenträgern in Kontakt, um allgemeine Maßnahmeninhalte oder die Zielgruppe zu thematisieren und die Erfahrung der Maßnahmenträger zu nutzen. Wenn etwa eine Maßnahme für Personen gesucht wird, über die dem SGB-II-Träger keine Informationen vorliegen, da Termine im SGB-II-Träger nicht wahrgenommen wurden, ist eine Zieldefinition problematisch. So führt eine Abstimmung zwischen Maßnahmen- und SGB-II-Träger dazu, flexibel auf die Bedarfe der Maßnahmenteilnehmenden reagieren zu können. Denn die Konkretisierung der Ziele vor Maßnahmenbeginn benötigt eine intensive Planung und Vorwissen, denn ein langfristig sinnvolles Arbeitsmarktprogramm muss der regionalen Wirtschaftsstruktur angepasst werden (Interview06Regio02). Darüber hinaus müssen die Maßnahmen über Haushaltsmittel finanzierbar sein und die gekauften Maßnahmenplätze auch bei längeren Planungszeiten mit der passenden Klientel besetzt werden. So ist Flexibilität in vielen Facetten für die Maßnahmenplanung von Bedeutung: »Und da sind die ARGEn sehr kreativ im Umgang mit dem Vergaberecht.« (Produktbetreuerin, Interview06Regio02)

Flexibilität in der späteren Maßnahmengestaltung kann auch auf die Teilnehmendenstruktur und deren neu auftretende oder bekanntwerdende Bedarfe reagieren. So wird bei der Maßnahmenplanung zunächst die Aktivierung vorrangig und überlegt, wie spezifische Personengruppen aktiviert werden können, bevor in Vermittlung und Fallmanagement abgewogen wird, wie die gesetzliche Realisierungsform für eine innovative aktivierende Maßnahme aussehen müsste. Häufig ist das grundsätzliche Vorgehen: »Erst die Konzepte und dann den Paragrafen, genau. Anders herum geht es gar nicht mehr.« (Fallmanagerin, Interview02Regio04) So ist für die AkteurInnen vor Ort nebensächlich, über welche gesetzliche Grundlage die Aktivierungsmaßnahme implementiert wird, solange die Aktivierung stattfinden kann (zum Beispiel Interview02Regio04).

Zusammenfassend ist im Bestreben, eine sehr arbeitsmarktferne Klientel zu aktivieren, die über die Standardinstrumente nicht erreicht werden kann, ein Abwägen zwischen dem Aktivierungsprinzip und der Rechtskonformität nötig. Denn in der Gestaltung und Erprobung von Maßnahmenelementen für diese zum Teil unbekannte Klientel kann, beispielsweise über eine eigenständige Vergabe von Arbeitsgelegenheiten durch den SGB-II-Träger, eine größere Autonomie erreicht werden. Darüber hinaus erhöht ein intensiver Austausch zwischen SGB-IIund Maßnahmenträger die Flexibilität und Anpassungsfähigkeit. Jedoch verfügen diese Instrumente oftmals nicht über den rechtlichen respektive institutionel- 
len Grad an Absicherung, wie ihn eine REZ-gesteuerte Ausschreibung im Normalfall bedeutet.

\subsubsection{Garantie der Rechtskonformität versus Bürokratisierung}

Die Zusammenarbeit mit den Regionalen Einkaufszentren wird häufig als Unterstützung seitens der SGB-II-Träger wahrgenommen, um rechtskonforme Vergaben in einer komplizierten Rechtslandschaft vorzunehmen. Gleichzeitig führt die Vergabe über das REZ jedoch auch zu einer Bürokratisierung und vermindert die Flexibilität in der Aktivierung von arbeitsmarktfernen Langzeitarbeitslosen, wie im Folgenden dargestellt wird.

\subsubsection{Rechtskonforme Vergaben}

Das REZ sieht seine wichtigste Bedeutung in der »Sicherstellung eines rechtsund verfahrenssicheren Vergabeverfahren[s; CF] durch Überwachung der einheitlichen Rechtsanwendung und Erteilung von Rechtsauskünften bei Vergabeverfahren sowie durch Koordinierung, Steuerung und Durchführung von Vergabeverfahren gemäß VOL/VOF \& VgV und GWB« (REZ Regio02 2010).

Eine bedarfsgerechte, vergaberechtskonforme und zugleich wirtschaftliche Beschaffung im Rahmen von wettbewerblichen und transparenten Vergabeverfahren wird damit in öffentlichen Ausschreibungen, Beschränkten Ausschreibungen und Freihändigen Vergaben bezweckt. Infolgedessen beziehen sich die REZ eindeutig auf die zugrunde liegenden gesetzlichen Auflagen. Weiter intendiert die zentrale Vergabe eine standardisierte Kontrolle der Maßnahmenqualität, der Wirtschaftlichkeit, der Beteiligungschance kleinerer oder nicht-ortsansässiger Maßnahmenträger und der Transparenz, um somit dem Gesetz gegen Wettbewerbsbeschränkung nachzukommen. Insofern werden Standardmaßnahmen und -verfahren zentral entwickelt. Insbesondere Standardmaßnahmen für arbeitsmarktnahe Personen im SGB-III-Bereich werden als hilfreiche Unterstützung von den SGB-II-Trägern wahrgenommen. Doch auch bei der eigenständigen Maßnahmenvergabe durch die SGB-II-Träger kann mit dem REZ die Passung des Maßnahmenwunsches mit gesetzlichen und institutionellen Vorgaben abgestimmt werden. Indessen stellen sich dem Innovationspotential jedoch bürokratische Hemmnisse, wenn etwa im Falle der sozialen Aktivierung, niederschwellige Maßnahmen geschaffen werden. Als Beispiel wird ein Projekt genannt, das in der Bewilligungsphase direkt von der BA geprüft und genehmigt wurde. Nach der Ausschreibung erging jedoch die interne Weisung, dieses Maßnahmenkonzept nicht erneut auszuschreiben (Interview04Regio04). 
»Das macht es uns umso schwerer, genau für den Personenkreis, der eben nicht unbedingt gleich möchte, der der wirklich einen ganz anderen Ansatz und nicht den Integrationsansatz hat, für die [Jugendlichen; $\mathrm{CF}$ ] entsprechende Maßnahmen vorzuhalten. Bloß, wir müssen so niedrigschwellig anfangen, ob wir wollen oder nicht.« (Bereichsleiterin, Interview04Regio04) $)^{44}$

Die Standardprodukte werden als vergleichsweise starr empfunden (zum Beispiel Interview02Regio04). Eingeschränkte Handlungsoptionen für die Angestellten der SGB-II-Träger durch Standardinstrumente und begrenzte Ressourcen, führen im Beratungsgespräch mit potentiellen Teilnehmenden häufig zu defensiver Konfliktvermeidung, doch nicht unbedingt zu zielgerichteter Vermittlung (Kolbe 2012).

Positiv bewertet wird von SGB-II-Trägern, wenn Standardprodukte überarbeitet werden und Erfahrungen aus der Praxis einfließen. ${ }^{45}$ Problematisch sei in diesem Überarbeitungsprozess dagegen der relativ hohe Zeitaufwand, der sich insbesondere durch die Einhaltung von Einkaufswellen ergebe, anstatt den Einkauf ab einem Stichtag zu ermöglichen (Interview02Regio04). Mangelnde Flexibilität durch eine notwendige langfristige Planung wird auch in einer anderen Studie kritisiert. Häufig würden Maßnahmenteilnehmende, für die eine Maßnahme beantragt wurde, zum Zeitpunkt der Maßnahmenrealisierung nicht mehr zur Verfügung stehen (Steinke et al. 2012: 29f.). ${ }^{46}$ Insgesamt erhalten SGB-IITräger über das REZ bei der Vertragsgestaltung "so eine Art Rückendeckung, um rechtssicher agieren zu können, denn wenn ich in so einer ARGE bin, die ja auch kommunal gesteuert ist, dann brauche ich irgendjemanden der mir sagt, wo es lang gehen kann, wie weit ich gehen kann.« (Zentraler Projektmanager, Interview03Regio07) Denn häufig steht der Bedarf für niedrigschwellige, nicht standardisierte Maßnahmen gemessen am Inhalt und den Bedürfnissen der Zielgruppe in einem zum Teil mehrere Rechtsbereiche tendierenden Kontext.

44 Dieser arbeitsmarktferne Personenkreis habe nach Aussage der Bereichsleiterin eine ausreichende Größe, um berücksichtigt zu werden. Als Beispiel werden Jugendliche angeführt, die teilweise vier bis sechs Jahre von Jobcentern betreut werden.

45 Vergleiche Kapitel 5.2.3 Rechtliche und finanzielle Rahmenbedingungen für die Schaffung der Maßnahmen Sozialer Aktivierung.

46 Um den Zeitaufwand zu minimieren, könnten die Jobcenter entweder versuchen, die Maßnahme in Form von Modellprojekten einzurichten oder in Abstimmung mit dem REZ verkürzte Fristen für Einzelfälle vorzusehen (Interview02Regio04). 
Eine flexible Handhabung des Vergaberechts kann Planungsprozesse verkürzen. Nebstdem können in die Maßnahmengplanung auch kommunale Belange einfließen, etwa die Unterstützung des kommunalen Maßnahmenträgers oder der kreiseigenen Volkshochschule. Die kommunalen Kosten reduzieren sich, wenn Ausgaben über die SGB-II-Eingliederungsmittel gedeckt werden. ${ }^{47}$

Gleichzeitig nutzen die Regionalen Einkaufszentren auch den verfügbaren Spielraum, um die SGB-II-Träger zu unterstützen und etwa in begründeten Einzelfällen, Maßnahmen zeitlich schneller zu vergeben. Insgesamt wird jedoch eine Tendenz der Bürokratisierung wahrgenommen:

»Ich merke aber jetzt auch, dass das REZ in den letzten Jahren bürokratisiert worden ist, mit entsprechenden eigenen Zielvorgaben, bestimmte Sachen nicht mehr so durchgegangen sind. Das merke ich schon, aber wir haben bei unseren Ansprechpartnern im REZ und ich bin auch selber öfters mal mit dem Leiter des REZes im Gespräch, da merke ich schon sehr viel Verständnis für unsere Situation und der bemüht sich auch alles zu erreichen, dem mache ich in keinster Weise irgendeinen Vorwurf.« (Zentraler Projektmanager, Interview03Regio07)

Eine rechtskonforme Vergabe, in der die SGB-II-Träger ausgeschriebene befristete Aufträge vergeben, soll die Effizienz der Beschäftigungsförderung erhöhen. Doch engere Zielvorgaben bedeuten vornehmlich eine stärkere Orientierung am arbeitsmarktnahen SGB-III-Klientel: ${ }^{48}$

»Aus Sicht kritischer Beobachter riskiert dies allerdings eine Engführung des Eingliederungsauftrags der Arbeitsmarktpolitik sowie Nachteile für diejenigen, die die größte Entfernung zum Ersten Arbeitsmarkt aufweisen (vgl. etwa Buestrich/Wohlfahrt 2004 oder Trube 2006). Im Hinblick auf die Differenzierung der Zielgruppen wird überdies eine »Zwei-Klassen-Behandlung« moniert (vgl. Burghardt 2005: 35), die gegenüber der vorher bestehenden institutionellen Trennung von Sozial- und Arbeitsverwaltung nur wenig Fortschritt bringe« (Bode 2011: 325f.)

47 Daher versuchen, aus Sicht des REZ in Regio02, die SGB II-Träger, das Vergaberecht zu umgehen, wählen dabei zertifizierte Bildungsmaßnahmen (FBB) oder Arbeitsgelegenheiten mit Qualifizierungsanteil um kommunale Maßnahmenträger auszulasten. Daneben können Pilotprojekte oder das Vermittlungsbudget nach § 45 SGB III dazu dienen, neue Elemente auszutesten und das Vergaberecht zu umgehen.

48 Vergleiche Kapitel 5.4.5 Verfestigte Arbeitslosigkeit. 


\subsubsection{Wirtschaftlichkeit und Wettbewerb}

Aus Sicht der SGB-II-Träger-Fachkräfte ist beim Maßnahmeneinkauf die wirtschaftlichste Möglichkeit nicht die sinnvollste, wenn Abstriche bei den eingekauften Leistungen gemachten werden müssen. Dabei sind SGB-II-Träger, wie alle öffentlichen Haushalte, zur Ermittlung des wirtschaftlichsten Angebotes verpflichtet.

Die Qualitätskontrolle eines Maßnahmenangebotes erfolgt über das schriftlich eingereichte Konzept. Die Bietereignung wird in verschiedenen Bereichen ermittelt: fachliche Vorerfahrungen, bisherige Integrationserfolge in den ersten Arbeitsmarkt, Schwierigkeiten die der regionale Einkauf mit diesem Maßnahmenträger hatte (vertragliche Sanktionen, Vertragskündigung etc.), das regionale Netzwerk des Maßnahmenträgers und die Ergebnisse der Teilnehmendenbefragungen. Die durch das REZ kommunizierten Maßgaben zur Angebotsbewertung scheinen dabei nicht allein an der Wirtschaftlichkeit und der Papierlage des Konzeptes ausgerichtet zu sein, wie in Vergabeunterlagen und Gesprächen geäußert, sondern auch Kennzahlen der früheren Zusammenarbeit einzubeziehen. Dennoch entsteht in den Reihen der SGB-II-Träger eher der Eindruck, dass eine vorherige erfolgreiche Zusammenarbeit und Warnungen vor Maßnahmenträgern, die sehr günstig sind und dafür erfahrungsgemäß weniger leistungsfähig sind, nicht in die Bewertung einfließen.

\subsubsection{Wirtschaftlicher Preisdruck schafft nicht-intendierte Nebeneffekte}

Bemängelt wird dementsprechend der hohe Preisdruck bei der Maßnahmenvergabe. Dies nicht nur von Maßnahmenträgern, sondern sehr häufig von den Jobcentern. Gemäß der fachlichen Steuerung (Interview06Regio02) seien nicht die Kosten, sondern das Verhältnis von Preis und Leistung entscheidend, denn die Ausgaben würden erst nach der Orientierung an den höchsten Leistungspunkten in die Prüfung einbezogen. Insofern wird auf das Wettbewerbsprinzip und den Angebotsvergleich weiterer Bieter im Vergabeverfahren verwiesen. In Bezug darauf dürfte einerseits die detaillierte Kenntnis der Zielsetzung und somit der Bemessungsgrundlage ausschlaggebend sein, für die Zufriedenheit der SGB-II-Träger. Allerdings wurde sehr häufig angesprochen, dass der Preis (abstrahierend von Güte und Umfang der Leistungen) das wichtigste Kriterium der REZ sei. Bereits in einer anderen qualitativen Befragung gaben SGB-II-TrägerBeschäftigte dies an (Steinke et al. 2012: 30). Denn wenn das jeweilige Jobcenter die Qualität der sich bewerbenden Träger beurteile, werde der vom Jobcenter favorisierte Träger nicht genommen, wenn er über einem bestimmten Preisniveau liege (so zum Beispiel Interview04Regio04). Hier könnte in der Angebots- 
prüfung des REZ ein Interpretationsspielraum vorliegen, der eine Verschiebung zu günstigeren Maßnahmenangeboten bewirkt, um öffentliche Mittel mit größtmöglicher Sparsamkeit zu verwenden. Insbesondere Jobcenter-Fachkräfte unterstellen indessen den Regionalen Einkaufszentren einen kurzfristigen Planungshorizont: Maßnahmen seien häufig zu Lasten von Ausstattung und Gehältern kostengünstiger. Durch niedrig kalkulierte Gehälter seien die vergebenen Anstellungen nur für Berufseinsteiger interessant, die in ein besseres Arbeitsverhältnis wechseln, sobald sich die Option ergebe. ${ }^{49}$ Häufige Personalfluktuationen aufgrund niedriger Bezahlung seien dagegen den Zielen des Jobcenters nicht zuträglich, denn die Maßnahmenqualität leide (besonders deutlich im Interview04Regio04).

\subsubsection{Trennung von Einkauf und SGB-II-Träger}

Weiter schränkt das Wettbewerbsprinzip die Schaffung und Umsetzung gemeinsamer Maßnahmenkonzeptionen von SGB-II- und Maßnahmenträgern ein. Erschwerend tritt hier die explizite Korruptionsprävention der Regionalen Einkaufszentren hinzu (BA 2010b), die im Beschaffungsprozess von Arbeitsmarktdienstleistungen auf eine konsequente Trennung von Einkauf und Bedarfsträger achtet. Wie in Kapitel 5.4.1 dargestellt, ist gerade für die Aktivierung einer arbeitsmarktfernen und häufig in seinen Bedarfen für die Erwerbsintegration unbekannten Klientel, der Austausch zwischen SGB-II- und Maßnahmenträgern vielfach erwünscht. Dies scheint der zentrale Scheideweg für innovative Elemente in der Maßnahmengestaltung zu sein, denn die Zusammenarbeit muss nicht unbedingt der ungewollten Aufrechterhaltung kommunaler Dienstleistungsgeflechte dienen, sondern kann neben den Standardmaßnahmen stärker die regionalen Bedürfnisse und Gegebenheiten fokussieren. In einem solchen Kontext entstehen nicht-standardisierte Formen, wie die soziale Aktivierung.

\subsubsection{Zuständigkeiten verschiedener Kostenträger geraten ins Blickfeld}

Ausschreibungen über die REZ bieten eine Trennung der Aufgabenbereiche zwischen SGB-II-Trägern und anderen Kostenträgern, die ebenfalls die SGB-IIKlientel betreuen. Zahlreiche SGB-II-Träger-Fachkräfte äußern, dass die heute zur Verfügung stehenden Maßnahmenmittel immer weniger ausreichen, für eine gleichbleibende Qualität und Quantität. Dagegen hätten die in 2005/2006 üppig

49 Rechtlich ist auch ein Ausschluss von Dumpingpreisen geboten (§ 16 Abs. 6 VOL/A 2009). 
zur Verfügung stehenden Mittel zu Begehrlichkeiten und einem Rückzug anderer Kostenträger geführt. Dies gelte für die Kommunen mit Leistungen nach dem SGB VIII (Kinder- und Jugendhilfe), SGB IX (Rehabilitation und Teilhabe behinderter Menschen), SGB XII (Sozialhilfe), aber auch weitere Kostenträger nach dem SGB V (Gesetzliche Krankenversicherung) und SGB VI (Gesetzliche Rentenversicherung). So wurden in diesen Jahren Maßnahmen »am Rande der Zuständigkeit - nicht der Legalität, aber der Zuständigkeit« (zentraler Projektmanager, Interview03Regio07) gefördert, etwa eine tagesstrukturierende Maßnahme oder teilstationäre Einrichtungen für Suchterkrankte.

»Das sind alles am Rande der Erwerbsfähigkeit operierende Maßnahmen wo man wirklich darüber streiten kann, ist das noch SGB II oder sind da nicht ganz andere Träger zuständig. Wohnungslosen-Projekte haben wir gesagt, wir nehmen was auf, wenn TingelTangel-Brüder, die mal hier in der ARGE sind mal da in der ARGE sind, den wollen wir ein Projekt anbieten, damit sie nicht nur Leistungen abziehen, sondern dann auch erstens mehr Geld haben, aber dann auch dafür Gegenleistung bringen müssen. Wenn man da genau hinguckt kann man da sagen, was ist da psychosoziale Hilfestellung, was ist kommunale Leistungsnotwendigkeit, da müssen wir jetzt seit zwei Jahren viel mehr hingucken.« (Zentraler Projektmanager, Interview03Regio07)

Viele für diese Klientel zuständige Kostenträger müssten sich über Kofinanzierungen zusammenschließen, um niederschwellige Maßnahmen im Rechtsbereich von mehreren Kostenträgern zu verwirklichen.

Darüber hinaus wird angeregt, umfassende Problemlagen der sehr arbeitsmarktfernen Klientel dadurch effektiver zu bearbeiten, dass in den Jobcentern Angebote diverser Kostenträger gebündelt werden, etwa die sogenannten Leistungen Dritter wie Schulden-, Sucht- und psychosoziale Beratung, ${ }^{50}$ aber auch Rehabilitation, Berufsberatung, Bewährungshilfe, Kinder- und Jugendhilfe, Sozialhilfe, Gesetzliche Rentenversicherung, denn »Wir wissen vielfach nichts voneinander.« (Fallmanager, Interview01Regio10). Eine qualitative Studie zu Dienstleistungsprozessen am Arbeitsmarkt ermittelte, dass zwei Fünftel der SGB-II-Leistungsbeziehenden Kinderbetreuungs-, Ehe-/Beziehungs-, Geld/Schulden-, Sucht-, psychosoziale und/oder sonstige soziale Probleme haben (Schütz et al. 2011: 314). Um effektiv an eine Beratungsinstanz vermitteln zu können, benötige auch das Fallmanagement Kenntnisse über die Abläufe. So

50 Die Identifizierung von notwendigen kommunalen Eingliederungsleistungen nach $\S 16 a$ SGB II ist ebenso Aufgabe der SGB II-Träger-Fachkräfte. 
»ist es nicht damit getan, dass ich sage, oh das könnte ein Reha-Problem sein, ich gebe das jetzt schon mal ab und dann sollen die sich darum kümmern. Ich muss schon Vorarbeiten leisten. [...] angefangen von der medizinischen Untersuchung gegebenenfalls ergänzt um eine psychologische Untersuchung und und und.« (Fallmanager, Interview01Regio10)

Die Beschäftigungsförderung hat sich dahingehend verändert, dass soziale Dienstleistungen im Rahmen der Fallbearbeitung und Abstimmungsprozeduren nun signifikant häufiger auftreten (Bode 2011: 319, Hielscher/Ochs 2009, Magnin 2006):

»Ich stelle aber schon seit langer Zeit fest, dass der Ansatz der BA falsch ist. Es geht hier nicht um Integration, es geht zunächst um soziale Begleitung und Entwicklung von Hilfsangeboten. Das heißt ich müsste eigentlich entweder von Haus aus Sozialpädagoge sein oder ich müsste schwerpunktmäßig in dieser Richtung mehr qualifiziert werden, um das zu tun, was ich hier tun muss.« (Fallmanager, Interview01Regio10)

Im Feld findet sich also entweder der Wunsch nach gezielter Schulung oder eine räumliche Bündelung kooperierender Dienste. Diese Aussagen decken sich mit Studienergebnissen zu den Dienstleistungsprozessen am Arbeitsmarkt, wonach soziale Problemlagen im Beratungsgespräch zwischen SGB-II- und SGB-IIIFachkräften und den Transferleistungsbeziehenden zwar besprochen wurden, aber die Vermittlungs- und Integrationsfachkräfte »nicht immer hinreichend als vorhandene Bedarfe an Unterstützungsleistungen Dritter weiterverarbeitet haben« (Schütz et al. 2011: 323). Dies wird von Schütz et al. und weiteren Studien mit Blick auf die Vermittlungsinteraktion auf professionelle Fehler im Sinne der unterlassenen Nachfragen zu Beratungs- und Unterstützungsbedarfen sowie teilweise auf mangelnde Angebotsstrukturen von Dritten zurückgeführt: Insgesamt gäbe es hier Verbesserungsbedarf für die Beratungsleistungen (Schütz et al. 2011: 323, Tisch, 2010, Baethge-Kinsky et al. 2007).

Zusammenfassend führt die Zusammenarbeit von SGB-II-Trägern und Regionalen Einkaufszentren im Vergabeprozess einerseits zur Unterstützung durch die REZ in einer komplizierten Rechtslandschaft. Andererseits wird der Einkaufsvorgang bürokratisiert und die Flexibilität in der Aktivierung von arbeitsmarktfernen Langzeitarbeitslosen erschwert. Eine Vergabe über das zuständige REZ soll die Effizienz der Beschäftigungsförderung insgesamt erhöhen. Dagegen bedeuten die hiermit verbundenen Zielvorgaben vornehmlich eine Orientierung an der arbeitsmarktnahen SGB-III-Klientel und damit einem kurzfristig effizienten 
Vorgehen. Im Sinne des Wirtschaftlichkeits- und Wettbewerbsprinzips handeln REZ und SGB-II-Träger. Allerdings fordern und praktizieren Letztere eine stärkere Berücksichtigung der angebotenen Leistung im Abgleich des Preis-Leistungs-Verhältnisses. Die Trennung des SGB-II-Trägers vom Beschaffungsprozess, als zentrale Maßgabe des REZ, zielt auf eine Korruptionsprävention. Doch eine Kommunikationsmöglichkeit zwischen SGB-II- und Maßnahmenträgern kann innovative Elemente für eine arbeitsmarktferne Klientel flexibel und passgenau gestalten. Ferner konfligieren Klientel-Zuständigkeiten mehrerer Kostenträger - etwa der Kommunen, der gesetzlichen Rentenversicherung oder der gesetzlichen Krankenversicherung. Diese unterschiedlichen Zuständigkeiten geraten durch die Regionalen Einkaufszentren stärker ins Blickfeld. Zur besseren Betreuung sollten Kooperationen gefördert werden. Bilanzierend wird die Zusammenarbeit mit den Regionalen Einkaufszentren von den Befragten als positiv betrachtet, gerade hinsichtlich der Entlastung in den Vergabeprozessen. Für die Entstehung von niederschwelligen und neuartigen Maßnahmen, die auch sozial aktivieren sollen, können sich die Ausschreibungs- und Vergabemodalitäten jedoch als Innovationssperren erweisen. Insofern ist es umso interessanter, dass die zentrale Steuerung der REZ, in Reaktion auf die von der Basis angefragten niederschwelligen Maßnahmen, im Laufe der Zeit ein institutionalisiertes Instrument für niederschwellige Maßnahmen etablierte.

\subsubsection{Begrenzung durch die vermittlungsorientierte Budgetierung}

Neben den gesetzlichen Regelungen begrenzt auch die Budgetierung die Schaffung niederschwelliger Maßnahmen. Zwar haben SGB-II-Träger formal Freiheiten und Handlungsspielräume in der Maßnahmenplanung, doch die Mittelfokussierung auf hohe Integrationsquoten kann eine Beschäftigungsförderung von Personen, die weiter vom Arbeitsmarkt entfernt sind, hemmen.

»Das Problem ist, wir haben keine Mittel mehr für diese Leute [dies meint weit vom Arbeitsmarkt entfernte Personen; CF]. [...] das erledigt der Gesetzgeber ganz elegant indem er einfach uns den Hahn zudreht und sagt, natürlich könnt ihr AGH machen, natürlich könnt ihr BEZ-Förderung machen, natürlich könnt ihr $16 \mathrm{f}$ machen, ihr müsst nur darauf achten, dass die alle sehr integrativ wirksam sind und ihr die Maßnahmen daraufhin prüft und den Rest könnt ihr ganz alleine machen.« (Zentraler Projektmanager, Interview03Regio07)

Denn seit 2007 sind die Zielvorgaben enger an Vermittlungserfolge in den ersten Arbeitsmarkt gebunden und beeinflussen damit auch die Verausgabung der Mit- 
tel. Hingegen war 2005/06 der Auftrag »den Menschen zu helfen« (zentraler Projektmanager, Interview03Regio07).

»Die Agentur guckt schon ganz genau, ob wir die Mittel vernünftig einsetzen und nach den Zielvorgaben des BMAS und der BA und da gibt es Zielvorgaben, da heißt es Ältere, Jugendliche, arbeitsmarktnahe Geschichten zu machen, das sind die Forderungen, und das sind Zielvorgaben die das BMAS über die BA umsetzt und die bis vor Ort in die einzelnen Agenturen reinkommen und die Agentur, schließt mit uns Zielvereinbarungen ab, mit unserer Geschäftsführung und sagt, ihr wisst ja die Zielvorgaben, so, dann kann sich kein Geschäftsführer leisten, wenn er diese Zielvorgaben nicht verfehlen will, hinzugehen ach das interessiert uns alles gar nicht, wir tun jetzt trotzdem sehr viele Mittel, die notwendig sind, weil 90 Prozent unserer Kunden eben nicht zielnah und integrationsnah sind, einfach für marktfernere Maßnahmen rein. Das ist quasi ausgeschlossen, das ist nicht verboten, das ist das Schöne an dem System. Du verbietest nichts, aber gibst Zielvorgaben so an, dass das andere sich automatisch erledigt.« (Zentraler Projektmanager, Interview03Regio07)

Wenn das gesellschaftspolitische Ziel eine Aktivierung der arbeitsmarktfernen Klientel beinhalte, so müssten auch in der Durchführung die Weichen für eine kontinuierliche, langfristige Arbeit mit nicht immer unmittelbar messbaren Erfolgen und größerem Zeitaufwand gestellt werden:

»Also steht der Staat vor der Frage, will ich das gesellschaftspolitisch anpacken, mit welchen Mitteln [...] Dieser ganze Krux: Verrechnung passive Leistungen, aktive Leistungen. [...] wenn man hingeht und sagt wir verrechnen eingesparte passive Leistungen durch passiven Leistungsbezug und wandeln die Hälfte um in aktive. Wären Millionen gespart und wir hätten riesen viel Geld für Arbeitsmarktpolitik. Das packt keiner an.« (Zentraler Projektmanager, Interview03Regio07)

Der Beschäftigungszuschuss wäre ein gutes Beispiel für die Verschiebung von passiven auf aktive Leistungen, da während der befristeten sozialversicherungspflichtigen Beschäftigung keine Lebensunterhaltskosten oder ähnliches ausgezahlt wurden. Auch die am Kalenderjahr orientierten Finanzierungszeiträume erweisen sich in der Praxis als negativ, da nicht ausgeschöpfte Mittel eines Haushaltsjahres zu Mittelkürzungen im folgenden Jahr führen. Wenn die Mittel ins nächste Jahr übertragen werden könnten, würden nicht kurzfristig zum Jahresende Maßnahmen aufgelegt werden, um die verbleibenden Gelder auszuschöpfen und Budgetkürzungen zu vermeiden. Überwiegend wurde in den befragten Regionen betont, dass mehr finanzielle Mittel für die Einrichtung niederschwelliger 
Maßnahmen notwendig wären. Weiter sei der Handlungsspielraum durch die geringe Bereitstellung der Verpflichtungsermächtigung eingeschränkt (zum Beispiel Interview02Regio04). Eine Verpflichtungsermächtigung ist nach $§ 75$ SGB II notwendig, wenn Maßnahmen den SGB-II-Träger zu Ausgaben in künftigen Haushaltsjahren verpflichten und ist dann zulässig, wenn der Haushaltsplan dazu ermächtigt.

Zusammenfassend kann das Maßnahmenbudget nur für eine sehr arbeitsmarktferne Klientel genutzt werden, wenn das Budget durch die internen Zielvorgaben nicht allein an die arbeitsmarktnahe Klientel gebunden ist.

\subsubsection{Die Eingliederungsquote begünstigt arbeitsmarktnahe Maßnahmen}

In enger Verbindung zur Budgetierung steht auch die Eingliederungsquote ${ }^{51}$ in den ersten Arbeitsmarkt, denn die SGB-II-Träger müssen ihre Mittel vornehmlich für die Erfüllung der Integrationsquoten aufwenden. Über sogenannte Zielvereinbarungen, zwischen dem Bundesministerium für Arbeit und Soziales und der Bundesagentur für Arbeit, im Einvernehmen mit dem Bundesfinanzministerium, werden die angestrebten Ergebnisse der Leistungserbringung jährlich ausgehandelt ( $§ 48$ Abs. 1 SGB II) und festgeschrieben. Auf lokaler Ebene werden diese durch Zielvereinbarungen zwischen der Trägerversammlung ${ }^{52}$ und den Geschäftsführungen der SGB-II-Träger auf die lokalen SGB-II-Träger übertragen. Hier festgehaltene Eingliederungsquoten sollen die SGB-II-Träger erfüllen und werden als Bemessungsgrundlage für das interne Controlling herangezogen. Eine Ausrichtung der arbeitsmarktpolitischen Instrumente an der Integrationsquote und das darauf ausgerichtete Controlling befördern dabei einen Creaming-Effekt, was eine Auslese derjenigen Personen bedeutet, die kurzfristig erfolgsversprechend in den ersten Arbeitsmarkt vermittelt werden können.

»Dann versucht man - weil es werden ja trotzdem immer Integrationen von uns gefordert und ne gewisse Erfüllung der Statistik wird gefordert, da muss man natürlich schauen, dass man die Leute irgendwo unterbringt die a können, $b$ auch wollen, dass man die ir-

51 Die Eingliederungsquote beschreibt den Anteil der Personen, die spätestens sechs Monate nach Maßnahmenende sozialversicherungspflichtig beschäftigt sind.

52 Die Trägerversammlung besteht aus Vertreterinnen und Vertretern der Agentur für Arbeit und des kommunalen Trägers (nach $§ 44 \mathrm{c}$ SGB II). 
gendwo priorisiert anstatt sich mit dem sogenannten, will nicht immer Bodensatz sagen, aber sich da abzuarbeiten, wo uns eigentlich klar ist, dass da eh nichts mehr geht, was unheimlich viel Zeit braucht, die wir eigentlich angesichts der steigenden Fallzahlen, was sich nächstes Jahr nochmal verschärfen wird, so nicht haben.« (persönliche Ansprechpartnerin, Interview02Regio06)

Die vorhandenen Zeit- und Mittelressourcen für die Beratung werden in der Praxis für einen Teil der Klientel verstärkt genutzt und dabei die Personen mit den geringsten Arbeitsmarktchancen im Verhältnis benachteiligt (Baethge-Kinsky et al. 2007: 62f.).

Zwar wird die Qualität laufender Maßnahmen über den Prüfdienst der Bundesagentur in Nürnberg, Prüfungen durch das zuständige REZ sowie die lokalen SGB-II-Träger gemessen. Doch im Feld wurde die Qualitätsmessung über die erreichten Eingliederungsquoten in den ersten Arbeitsmarkt häufiger vorgebracht. Da Maßnahmen Sozialer Aktivierung vordergründig auf eine weit vom Arbeitsmarkt entfernte Klientel zielen und die Arbeitsmarktintegration eher ein langfristiges Ziel darstellt, ist die Eingliederung in Arbeit nicht unbedingt in der ersten Maßnahme möglich. Deswegen erreichen niederschwellige Maßnahmen strukturell eine schlechtere Qualitätsbewertung, gemessen an den Eingliederungsquoten. Hier wurde bei der Erhebung häufig der Wunsch geäußert, auch andere objektivierbare Daten für die Qualitätsmessung einzubeziehen und inhaltliche Aspekte in den Vordergrund zu stellen. Denn aktuell muss der SGB-II-Träger in seiner Maßnahmenplanung eruieren, ob eine ausreichende Integrationsquote in anderen Maßnahmen erzielt werden kann, um die niedrigen Eingliederungsquoten der niederschwelligen Maßnahmen auszugleichen und diese dadurch anbieten zu können.

Als Beispiel für den Umgang mit den Integrationsquoten dient Regio11. Hier sei die regionale Arbeitsmarktlage insofern bedrückend, als Ausbildungsplätze und Stellen in Bäckereien, Metallbetrieben etc. zur Verfügung stehen, doch zu wenige Personen diese Stellen besetzen könnten. Insbesondere junge Erwerbsfähige sind in den vergangenen Jahren abgewandert, da es damals im Landkreis kaum vakante Stellen gab. Wegweisend für die Maßnahmenplanung ist auch im Jobcenter der Regio11 die zentral vorgegebene Eingliederungsquote. Nach Einschätzung der Teamleiterin (Interview03Regio11) seien die zu erfüllenden Quo- 
ten nicht realistisch. ${ }^{53}$ Im Vorjahr wurde die Integrationsquote nicht erreicht, obwohl gezielt auf Maßnahmen zurückgegriffen wurde, die die Integrationsquote vorübergehend verbessern. Wenn ein SGB-II-Träger im Vorjahr eine gute Integrationsquote erreicht hat, wird die anvisierte Quote für das laufende Haushaltsjahr beispielsweise um ein halbes Prozent erhöht. Die Regionaldirektion reagiere darauf mit einer Erhöhung um ein weiteres Prozent. Bei Nicht-Erreichen der Eingliederungsquote werden Erklärungen und Nachweise verlangt, die bei den vorgesetzten Ebenen meist auf Unverständnis stoßen. Dies wird auch von anderen SGB-II-Träger-Fachkräften thematisiert, in aller Deutlichkeit in folgender Sequenz:

»Und dann kommt aus diesem System immer wieder der große Daumen, warum, warum, warum [...] und das das funktioniert einfach nicht. [...] Ich habe den Eindruck, dass die Politik über die Regionaldirektion sehr viel stärker in die Fläche runter drückt und da dort häufig Leute sitzen, die nie im Schützengraben gelegen haben, nie Frontkämpfer gewesen sind, wissen die auch gar nicht, wie sich das anfühlt hier immer vor der Front zu stehen.« (Fallmanager, Interview01Regio10)

Auf der anderen Seite betont die Teamleiterin in Regio11 die Notwendigkeit einer Arbeitsmarktintegration, die, wenn nicht lokal, so doch im Bundesgebiet erreicht werden sollte. Dies ist eine klare Vorgabe der Institution, in der sie sich bewegt und obwohl sie in der Interviewpause Kritik an dieser Vorstellung äußert, setzt sie die >unrealistischen` Vorgaben um und hält diese in ihrer Grundintention für zielführend.

Ferner steht die Orientierung an der Integrationsquote auch dem Ziel der Bundesagentur für Arbeit entgegen, »Langzeitbezieher zu aktivieren und deren Integrationschancen zu erhöhen« (BA 2013e: 23).

»Der alte Mechanismus des >creaming the poor`, der für die Armutsverwaltung insgesamt (WZB \& infas 2005: 188), wie für die Arbeitsvermittlung galt (Eberwein \& Tholen 1986: 134 ff., 387 ff.), wird so durch Regime der Aktivierung nicht - wie es möglich wäre und gesetzlich vorgeschrieben ist ( $\S 1$ Abs. 1 S. 4 SGB II und 1 Abs. 2 und 6-8b SGB III )beseitigt, sondern verstärkt (Stafford 2002: 57, 66; Dahl \& Pedersen 2002: 69, 72).« (Bieback 2009: 202)

53 Das für die Leistungsmessung der Erwerbsintegration auch regionale Rahmenbedingungen einbezogen werden müssen, wurde bereits von Blien und KollegInnen konstatiert (Blien/Hirschenauer/Kaufmann/Moritz/Vosseler 2011). 
So hat die Bildung von Kundensegmenten mit einem daran orientierten Handeln negative Auswirkungen auf den Integrationserfolg, auch da die Segmentierung eine individuelle Wahrnehmung und Betreuung erschwert (Bieback 2009: 202). $\mathrm{Ob}$ und inwiefern sich die Qualitätsmessung durch die Einführung einer Aktivierungsquote für Langzeitarbeitslose in der Praxis sowie die weiteren Indikatoren der Verwirklichung der Steuerungsziele ändern wird, liegt außerhalb des Zeithorizontes dieser Studie. Die im Bund-Länder-Ausschuss 2011 verabschiedeten "Gemeinsame(n) Grundlage(n) der Zielsteuerung SGB II« (BA 2013e: 19f.) für SGB-II-Träger, die eine Erweiterung der Steuerungs- und Qualitätsziele vornehmen ${ }^{54}$ wurden im Erhebungszeitraum noch nicht von den SGB-II-TrägerBeschäftigten thematisiert.

Zusammengefasst befördert eine überwiegend an Integrationsquoten orientierte Beschäftigungsförderung den Creaming-Effekt, indem vor allem arbeitsmarktnahe Personen mit einem geringeren Unterstützungsbedarf beraten und vermittelt werden. Denn Maßnahmen für eine arbeitsmarktferne Klientel haben strukturell schlechtere Integrationsquoten, da hier vielfach nur eine Erwerbsintegration in langfristiger Perspektive möglich ist.

\subsubsection{Verfestigte Arbeitslosigkeit: Eine arbeitsmarktferne Klientel}

Zwischen den auf Erwerbsintegration ausgerichteten Zielvereinbarungen und den daraus folgenden Parametern für Aktivierungsinstrumente, die eine sehr arbeitsmarktferne Klientel unterstützen sollen, besteht ein Spannungsfeld. In Folge der ausgeweiteten Erwerbsfähigkeitsdefinition sind 2005 mehr als 90 Prozent der ehemaligen Sozialhilfeempfangenden als erwerbsfähig eingestuft worden, was zu einer Ausdehnung der zu aktivierenden Klientel auf fast eine Million führte (Konle-Seidl/Lang 2006: 20). »Der Markt ist erschöpft, so. Die treten dann auch in Konkurrenz ja zu den anderen, die also diese Hemmnisse nicht haben, die aber den gleichen Arbeitsmarkt nachfragen und das ist ein ganz großes Problem dabei.« (Fallmanager, Interview01Regio10) Bezogen auf den SGB-II-Rechtskreis berichten die Befragten in der vorliegenden Studie überwiegend von einer sehr weit vom Arbeitsmarkt entfernten Klientel, mit unterschiedlichsten finanziellen, gesundheitlichen und sozialen Problemlagen. Beschrieben werden Personen mit

54 Steuerungsziele sind - neben der Integrationsquote - die Summe der Leistungen zum Lebensunterhalt, der Bestand an Langzeitleistungsbeziehern sowie Qualitätsziele über einen Index aus der Kundenzufriedenheit und Prozessqualität (BA 2013e: 20). 
einer geringen schulischen oder beruflichen Qualifikation, Schulden, Suchttendenzen, die psychische und physische Einschränkungen aufweisen und insbesondere in Hilfstätigkeiten einsetzbar sind. ${ }^{55}$ Nicht nur dieses Forschungsunterfangen, sondern auch weitere wissenschaftliche Arbeiten haben ähnliche Problemlagen ermittelt (Kaltenborn/Kaps 2013 sowie Henkel et al. 2009: 59ff., Schubert et al. 2013), zudem führen Henkel et al. und Schubert et al. auch psychische Belastungen wie Angstzustände und Depressionen unter den Leistungsbeziehenden an. Eine Teamleiterin aus dem empirischen Material der vorliegenden Studie, charakterisiert SGB-II-Leistungsbeziehende mit psychischen Problemlagen und sozialen Aktivierungsbedarf wie folgt:

»Wenn ich hier so manche über den Flur schleichen sehe, auch die Leute die besonders frech sind, das ist nur eine Wand hinter der sie sich verstecken, weil sie Angst haben. Wenn man die genau anguckt: die zucken so in sich zusammen, die werden so klein mit Hut. Was einem eigentlich schon wieder leidtun kann.« (Teamleiterin, Interview03Regio11)

In eine ähnliche Richtung tendiert eine Forschungsaussage, die besagt, »dass die Dienstleistungen am Arbeitsmarkt für Menschen erbracht werden, die manchmal mehr als ein Qualifikations- oder Altersproblem auf dem Arbeitsmarkt haben.« (Schütz et al. 2011: 307f.). Vermittlungs- und Beratungsfachkräfte zeichnen insgesamt das Bild einer wachsenden Kluft zwischen den Arbeitsmarktanforderungen und der Qualifikation sowie körperlichen Leistungsfähigkeit der Leistungsbeziehenden (Ames 2008: 29ff., Schubert et al. 2013). Auch in den Erstgesprächen mit als arbeitsmarktfern klassifizierten Leistungsbeziehergruppen, den integrationsfernen Kunden (IF) und Personen mit festgestelltem Stabilisierungsbedarf (IG) werden Themen der Leistungsfähigkeit und Gesundheit anteilig häufiger besprochen, wohingegen Stellensuche-Thematiken mit IG-Personen mit Abstand am wenigsten besprochen wurden (Schütz et al. 2011: 71). Gut jede vierte Person im SGB-II-Bezug der Schütz et al.-Studie berichtet von eigenen schweren Krankheiten: einige Transferleistungsbeziehende bekennen sich zu Erfah-

55 Es ist eine deutlich häufigere Nutzung von vermittlungsunterstützenden Leistungen nach $\S 16 \mathrm{a}$ ff. SGB II durch Leistungsbeziehende im SGB II-Rechtskreis als im SGB II zu verzeichnen: jede/r Siebte hatte eine Schuldnerberatung, jede/r Sechste eine ärztliche oder psychologische Eignungsbegutachtung und auch Suchtberatung beziehungsweise eine psycho-soziale Beratung wurden in Anspruch genommen (Schütz et al. 2011: 306). 
rungen mit Wohnungslosigkeit, Alkohol- und Drogenproblemen sowie Haftstrafen (Schütz et al. 2011: 305). ${ }^{56}$ Ein ähnliches Bild der Problemlagen liefert die »Erhebung von Ansätzen guter Praxis zur Integration Suchterkrankter ins Erwerbsleben im Rahmen des SGB II« (Henkel et al 2009: 59). Daneben ist Schubert et al. zufolge ein Großteil der Erwerbslosen mit teils chronischer psychischer Erkrankung dazu fähig, drei Stunden täglich erwerbstätig zu sein und entspricht damit der Klientel des SGB-II-Rechtskreises. Doch gleichzeitig begründen psychische Erkrankungen immer häufiger Erwerbsausstiege der gesundheitsbedingten Frühverrentung (Schubert et al. 2013: 79).

»Wir haben nach meiner Erfahrung hier einen Personenkreis der bis zu 60 bis 70 Prozent aus der früheren Sozialhilfe gekommen ist, der eigentlich von Ausnahmen abgesehen nie einen Gedanken an Arbeit verschwenden musste, der Teil der vom alten Arbeitsamt gekommen ist, nämlich die Arbeitslosenhilfe-Personen, das sind ja auch schon zum Teil stark verfestigte Arbeitslosigkeiten gewesen, die sich nicht sehr stark von dieser Personengruppe der früheren Sozialhilfebezieher unterschieden hat und von daher sind Ansätze so wie sie im SGB III definiert sind, als Hilfen nur sehr schwer bis gar nicht brauchbar« (Fallmanager, Interview01Regio10).

Insgesamt zeigt sich eine Orientierung der beschäftigungsfördernden Instrumente im SGB II an dem SGB III. Dies wird auch an dem $\S 16$ Abs. 1 SGB II deutlich, der auf die Eingliederungsleistungen im SGB III verweist, die in der Maßnahmenausgestaltung auch für SGB-II-Leistungsbeziehende verwendet werden können und sollen. »Das SGB III ist letztendlich die Bibel für die Arbeitsförderung.« (Produktbetreuerin, Interview06Regio02). Darüber hinaus werden jedoch auch SGB-II-spezifische Beschäftigungsförderungen wie die AGH nach $\S 16 \mathrm{~d}$ SGB II ermöglicht. Durch den Verweis der Beschäftigungsförderung auf die Instrumente im SGB III ergeben sich nach Aussage der Produktbetreuerin keine negativen Folgen hinsichtlich des Handlungsspielraums oder der Finanzierungsoptionen (Interview06Regio02). Allerdings wird seitens der SGB-II-TrägerFachkräfte deutlich, dass damit auch die Zielvorgaben und Handlungsorientierungen aus dem SGB III auf die SGB-II-Klientel übertragen werden:

56 Knapp jede fünfte Person berichtet über weitere Faktoren, die die Erwerbsaufnahme behindern: Familien- beziehungsweise Partnerschaftsprobleme, Geld- und Schuldenproblematiken sowie schwere Erkrankungen naher Familienangehöriger (Schütz et al. 2011: 305). 
»Denn das Regelwerk [des SGB II; CF] und da würde ich also auch Kritikern der BA recht geben, hängt immer noch sehr lastig an SGB-III-Kunden dran, man sieht das auch an der Zielvorgabe, alles integrationsnah, möglichst kurze Förderdauer, möglichst viele Menschen mit wenig Geld in eine Integration zu führen. Das passt aber nur teilweise auf unseren Kundenbereich.« (Zentraler Projektmanager, Interview03Regio07)

Zwar sei eine hohe Integrationsquote im SGB II bei entsprechender Konjunktur und dem Vorhandensein marktnaher Personen in der zu aktivierenden Klientel möglich, doch gerade die letztere Randbedingung sei primär im SGB III vorzufinden. Für den SGB II-Bereich werden daher auch längere Maßnahmen benötigt (über neun bis zwölf Monate), für die sich die Maßnahmenplanung rechtfertigen müsse, da die Arbeitsmarktintegration auf kurze Maßnahmenverweildauern ausgelegt ist. Doch dies sei notwendig,

»weil die Leute, die hole ich ganz von unten ab und die möchte ich in einer Maßnahme durchführen, wegen der Kontinuität und nicht in sechs verschiedenen und mich nicht immer wieder rechtfertigen, dass das jedes Mal eine höhere Maßnahme ist, weil der muss ja in drei Monaten weitergekommen sein, wenn nicht, hat irgendeiner einen Fehler gemacht. Da hat keiner einen Fehler gemacht, das ist nämlich die Zeitdauer, die dafür da ist um das vernünftig durchzuführen.« (Zentraler Projektmanager, Interview03Regio07)

Insofern sind die Finanzierungsbedingungen sowie eine auf das marktnahe SGBIII-Klientel ausgerichtete Qualitätssicherung die handlungseinschränkenden Komponenten, durch die die Anbindung der Arbeitsmarktinstrumente des $\mathrm{SGB} \mathrm{II}^{57}$ an das SGB III für die Arbeitsvermittlung und Fallmanagement in den SGB-II-Trägern teilweise problematisch ist. Schönig stellt fest, dass ein durch die Aktivierungspolitik gestiegener Rechtfertigungsdruck als langfristig-konzeptionelles Phänomen besteht und diese Politik auch eine monetäre Bewertung der Ergebnisse Sozialer Arbeit einfordert (Schönig 2006: 34). Demgemäß wurden 2012 zunehmend Instrumente mit arbeitsmarktnäheren Ausrichtungen durch die Jobcenter eingesetzt (BA 2013e: 17). Die stark an Integrationszahlen gemessene Qualitätssicherung beeinträchtigt daher den Einkauf von arbeitsmarktfernen Maßnahmen und unterläuft so eine nachhaltige Aktivierung Langzeitarbeitsloser:

57 Das SGB II ist durch den $\S 16$ SGB II an das SGB III gebunden, indem es erwerbsfähigen Hilfebedürftigen den Zugang zu einem Großteil der SGB III-Instrumente als Ermessensleistungen ohne Rechtsanspruch eröffnet. 
»man muss uns mehr Zeit zum Atmen geben und darf nicht mit Qualitätsmanagement und Controlling zu sehr hinterher sein, in einer sehr kurzen Zeit Erfolge zu dokumentieren, sondern einfach Lust zu geben mit den Leuten zu arbeiten und unsere Kunden, die interessiert keine Statistik und auch kein Controlling, denen die Zeit zu geben das vernünftig zu machen. Das bekommen wir hin, aber es ist schwer und wir bekommen es in zunehmenden Maße nicht mehr hin, weil uns die Mittel fehlen.« (Zentraler Projektmanager, Interview06Regio10)

Die durch institutionelle Barrieren bedingte, geringe Nutzung von niederschwelligen Maßnahmen dürfte auch in einem Zusammenhang stehen, mit der Dienstleistungsunterversorgung der SGB-II-Leistungsbeziehenden, die den höchsten Unterstützungs- und Beratungsbedarf haben. Allerdings stellt eine wissenschaftliche Untersuchung überzeugend dar, dass gerade der arbeitsmarktfernsten Klientel eine niedrigere Beratungszeit und Maßnahmenfrequenz zukommt, was wiederum auf die Kundendifferenzierung zurückgeführt werden kann (Behrend 2007). Die Förderung der sozialen Aktivierung hat das Potential, dem von Baethge-Kinsky et al. konstatierten Umstand entgegenzuwirken, dass sich der Zeit- und Mitteleinsatz in der Vermittlungspraxis zu Gunsten der arbeitsmarktnahen und zu Lasten der arbeitsmarktfernen Klientel verteilt (Baethge-Kinsky et al. 2007: 62f.). Insofern wünschen sich die SGB-II-Träger-Fachkräfte, dass die politisch motivierten BA-Zielvorgaben langfristiger ausgelegt werden, denn eine kurzfristige Steuerung führe auch im Fallmanagement zu Verwirrung, da bewährte Projekte aufgrund neuer Zielsetzungen und Mittelkürzungen nicht weitergeführt werden könnten:

»Wir haben auch Handlungsspielräume, $[\ldots]$ wir haben Gesetzesvorgaben, die wir nutzen können, wir haben alles, aber wir brauchen eine langatmigere Politik, keine ständigen Veränderungen, keine immer neuen Projektorientierungen, nicht immer neue Zielvorgaben, die sich immer wieder ändern, sondern wir brauchen eine Langfristigkeit in der Arbeitsmarktpolitik und man muss den Leuten vor Ort einfach die Zeit geben.« (Zentraler Projektmanager, Interview03Regio07)

Dem stehen andere Entwicklungen angeblich entgegen: Eine Bürokratisierung der Regionalen Einkaufszentren und die stärkere Orientierung an der Integrationsquote sei insbesondere in den vergangenen Jahren spürbar:

»Mittlerweile hat sich die Schraube genau anders herum gedreht und ich habe den Eindruck die Politik geht jetzt und sagt, das Fass, dass wir da aufgemacht haben im SGB II, das hätten wir eigentlich gar nicht aufmachen sollen, jetzt sind wir irgendwo bis an den 
Grund rangekommen und fischen da unten im Trüben rum und merken wie schwierig das ist und mit wie viel Geldmitteln wir da reinbuttern, bis wir die Leute auffangen müssen, da machen wir das Fass besser wieder zu und schöpfen da oben das einigermaßen Frischwasser ab und unten, das bleibt drin. Damit ist das SGB II aber aus meiner Sicht ad absurdum geführt, denn dann hätte man auch die alten Systeme belassen können SGB III und SGB XII, mit Sozialhilfe und Arbeitslosenhilfe.« (Zentraler Projektmanager, Interview03Regio07)

Zusammengefasst kommt die Praxis der Arbeitsverwaltung mit einer sehr arbeitsmarktfernen Klientel in Kontakt, die es gemäß Aktivierungsdiskurs zu aktivieren gilt. Diese Klientel ist zwar durch physische, psychische, soziale und finanzielle Problemlagen geprägt, doch eine berufliche Leistungsfähigkeit von drei stündiger Arbeit täglich, kann diese Klientel erbringen. Gleichzeitig erweisen sich Arbeitsmarktinstrumente, die kurzfristig in Erwerbstätigkeit integrieren möchten, als unpassend für diese Personen. So ist es bedeutsam, politisch zu definieren, ob sich die Aktivierung auf alle erwerbsfähigen Sozialleistungsbeziehenden erstrecken soll und welche Maßnahmeninstrumente für die sehr arbeitsferne Klientel eingesetzt werden sollen. Dementsprechend ist die Wirksamkeit der Instrumente von Bedeutung, jedoch vor allem die Aufnahme dieser Zieldefinition in die Zielvereinbarungen, eine entsprechende Mittelausstattung sowie eine Berücksichtigung im Controlling.

\subsubsection{Bottom-up-Entwicklung Sozialer Aktivierung versus Top-down-Steuerung der Beschäftigungsförderung}

Seit 2004 wird die Arbeitsverwaltung in Deutschland im Rahmen einer wirkungsorientierten Zielsteuerung reguliert, das heißt einerseits über die gesetzlichen Grundlagen im Zweiten und Dritten Sozialgesetzbuch sowie andererseits über innerinstitutionelle Zieldefinitionen (Zielvereinbarungen), deren Einhaltung über das Controlling geprüft wird. Die Top-down-Steuerung vom Bundesministerium für Arbeit und Soziales über die Bundesagentur für Arbeit bis hin zu den örtlichen SGB-II-Trägern setzt besonders auf die arbeitsmarktnahen Instrumente der Erwerbsintegration. Denn die Rahmenbedingungen erschweren die Implementierung von Maßnahmen für sehr arbeitsmarktferne Personen. Dennoch waren im Sample dieser Studie zahlreiche Geschäftsführungen und Teamleitungen von SGB-II-Trägern von der Notwendigkeit geeigneter niederschwelliger Maßnahmen für eine spezifische Klientel im SGB II überzeugt. So konnten in offe- 
nen Maßnahmenformen ${ }^{58}$ bereits Maßnahmen Sozialer Aktivierung trotz hinderlichen Rahmenbedingungen durchgeführt werden. Wobei den übergeordneten Instanzen eine zumindest tolerierende Rolle zukommt. Doch die Entstehungsgeschichte des Aktivcenters ${ }^{59}$ zeigt, dass zahlreiche Maßnahmen trotz guter $\mathrm{Zu}$ sammenarbeit mit den zuständigen REZ erst mit der Schaffung des Aktivcenters nach § 46 SGB III im Jahr 2010 realisiert werden konnten.

Die Bundesagentur für Arbeit zeichnet sich durch eine sehr ausgeprägte Topdown-Steuerung aus, in der zentrale Vorgaben über standardisierte Verfahren und Zielvorgaben ausgegeben und in einer Qualitätssicherung deren Einbringung abgesichert wird. Zwar ist formal eine Konditionalsteuerung erreicht, da sich die einzelnen Jobcenter an individuellen Zieldefinitionen ausrichten müssen, doch werden diese meist über quantifizierbare Eingliederungsquoten und nicht qualitative Zieldefinitionen erreicht, anhand derer die Qualität von niederschwelligen Maßnahmen, die sozial aktivieren sollen, bemessen werden könnten. Denn die zentrale Steuerung der Bundesagentur für Arbeit und deren auf eine marktnahe SGB-III-Klientel ausgerichteten Vorgaben in der Maßnahmengestaltung schränken die Jobcenter in der Gestaltungsfreiheit und Zielausrichtung auf Langzeitarbeitslose und somit deren Vermittlungserfolg ein. So sind Fachkräfte in der Vermittlungsberatung überwiegend institutionell gebunden, unterliegen also rechtlichen, organisatorischen und auch zeitlichen Grenzen, so dass ihr Handlungsraum in Vielem vorstrukturiert ist (Schütz et al. 2011: 316).

Ein denkbarer Schritt zur Ausweitung innovativer Elemente, die durch die Praxis der Vermittlungs- und Fallmanagement-Fachkräfte geschaffen werden könnten, wären erweiterte Handlungsspielräume in der Vermittlungspraxis. Ein solches Vorgehen wird auch in dieser Studie von einem Fallmanager gewünscht, der in der Vergangenheit als Arbeitsberater über ein bestimmtes Maßnahmenbudget aus dem Eingliederungshaushalt für Bildungsmaßnahmen verfügte (Interview01Regio10). Ein solches Instrumentarium wurde bereits geschaffen mit dem Vermittlungsbudget nach § 45 SGB III, § 16 Abs. 1 SGB II i.V.m. § 45 SGB III, das den lokalen SGB-II-Trägern mehr Entscheidungsspielräume verleiht, um nicht nur standardisierte Förderleistungen zu vergeben (Steinke et al. 2012: 8). Das Vermittlungsbudget wurde jedoch kaum abgerufen und stattdessen formalisiert nach »ermessenslenkenden Weisungen« eingesetzt, an denen sich

58 Dargestellt im Kapitel 5.2 Rechtlicher und finanzieller Rahmen für die Schaffung sozial aktivierender Maßnahmen.

59 Dargestellt im Kapitel 5.3 Das Aktivcenter: Professionalisierung und Zeichen einer Bottom-up-Entwicklung. 
die Geschäftsführungen der Agenturen für Arbeit und SGB-II-Träger orientierten (Steinke et al. 2012). Folglich hemmt insbesondere die Top-down-Kultur innovierende Elemente in ihrer Realisierung. Weiterhin weichen die umsetzenden AkteurInnen von den institutionellen Vorgaben in ihren Handlungen ab: Dies mag durchaus im Eigeninteresse liegen, aber auch im Interesse der Leistungsbeziehenden (Schütz et al. 2011: 316). Darin bestätigen die Befunde die Untersuchung zur Street-level-Bürokratie: Markant ist die hohe Einzelfallorientierung der Vermittlungsfachkräfte verbunden mit der Bereitschaft, gesetzliche und institutionelle Vorgaben auch pragmatisch zu nutzen, um auf individuelle Problemlagen flexibel reagieren zu können (Schütz et al. 2011: 316).

Insgesamt wurden im Kapitel 5.4 Spannungsfelder in der Arbeitsverwaltung aufgezeigt, die durch das Zusammentreffen der niederschwelligen Klientel mit den Prämissen des stark arbeitsmarktorientierten Aktivierungsdiskurses entstehen. So ist es für SGB-II-Träger notwendig, zwischen dem Aktivierungsprinzip und der Rechtskonformität abzuwägen, wenn passgenaue und flexible Instrumente für eine sehr arbeitsmarktferne oder nahezu unbekannte Klientel geschaffen werden. Den Vergabeprozess über die Regionalen Einkaufszentren durchzuführen, bedeutet einerseits eine Unterstützung im komplexen Rechtsfeld. Andererseits werden die Instrumente dann an einer zeitnahen Erwerbsintegration ausgerichtet, die eher bei einer arbeitsmarktnahen Klientel realisierbar ist. Erschwerend für die Anwendung innovativer Maßnahmeninstrumente (für eine arbeitsmarktferne Klientel) ist ein Budget, das durch interne Zielvorgaben an die arbeitsmarktnahe Klientel gebunden ist. Somit befördert eine überwiegend an Integrationsquoten orientierte Arbeitsverwaltung, dass arbeitsmarktnahe Personen bevorzugt betreut werden. Während arbeitsmarktferne Personen durch die Aussicht auf eine unumgänglich scheinende schlechtere Integrationsquote weniger intensiv betreut werden.

So wird deutlich, dass SGB-II- und Maßnahmenträger mit einer teilweise sehr arbeitsmarktfernen Klientel in Kontakt treten. Diese gilt trotz vielfältiger Problemlagen jedoch als dazu fähig, mindestens drei Stunden täglich erwerbstätig und integrationsfähig zu sein. Die SGB-II- und Maßnahmenträger-Fachkräfte dieser Studie berichten allerdings, dass arbeitsmarktnahe Beschäftigungsförderungsansätze für diese arbeitsmarktferne Klientel nicht geeignet sind. Vielmehr wird eine langfristige arbeitsmarktpolitische Definition gefordert, die expliziert, inwiefern eine arbeitsmarktferne Klientel aktiviert werden soll. Dementsprechend wird vielfach formuliert die Zielvereinbarungen (von dem Bundesministerium für Arbeit und Soziales bis hin zu den SGB-II-Trägern), die Kernpunkte des Controllings sowie die Budgetierung an diese Definition anzupassen. 
Seit 2004 reguliert die wirkungsorientierte Steuerung top-down, vom Bundesministerium für Arbeit und Soziales, über die Bundesagentur für Arbeit bis hin zu den SGB-II-Trägern. Es dominieren auf zeitnahe Erwerbsintegration zielende Instrumente und entsprechende Eckpunkte der Qualitätssicherung. Dennoch konnten sich bottom-up Maßnahmen Sozialer Aktivierung etablieren. Die zunächst in offenen Maßnahmenformen angelegten Maßnahmenelemente, konnten ab 2010 in einem standardisierten und erleichterten Verfahren als Aktivcenter beschafft werden. Die Entstehung des Aktivcenters weist auf die Bedeutung der arbeitsmarktfernen Klientel hin. Die Praxis der Arbeitsverwaltung hat das hierarchisch darüberstehende Aktivierungskonzept dahingehend erweitert, was in den nachfolgenden Ausführungen zu zeigen sein wird.

\section{5 „WIR MÜSSEN AN DIE PERSÖNLICHKEIT HERAN «: MASSNAHMENFUNKTIONEN}

»Da haben wir erkannt, eine AGH löst die Probleme nicht, sondern wir müssen an die Persönlichkeit heran und nicht an das Berufsfeld « (Interview03Regio07). Mit dieser Aussage stellt der zentrale Projektmanager eines SGB-II-Trägers heraus, dass soziale Aktivierung wesentlich mehr umfasst als eine Erwerbsintegration. Um im Rahmen der unter den Leitlinien des Aktivierungsdiskurses wirkenden Beschäftigungsförderung eine besondere Form niederschwelliger Maßnahmen für meist langzeitarbeitslose Personen mit Problemlagen - die Maßnahmen Sozialer Aktivierung - zu beschreiben, wurde aufgezeigt, dass insbesondere die Nachfrage durch die lokalen SGB-II-Träger-Fachkräfte und deren Wunsch, nicht nur arbeitsmarktnahe Personen zu vermitteln, sondern insbesondere arbeitsmarktferne erwerbslose Leistungsbeziehende anzusprechen, dazu führte, dass trotz innovationshemmender Rahmenbedingungen die neue Maßnahmenform bottom-up entstand. Die Maßnahmen erfüllen Funktionen, die über die bloße Ansprache und Hinführung zum Arbeitsmarkt hinausweisen. Diese Funktionen werden auf drei verschiedenen Ebenen rekonstruiert: 1.) wird die Ebene der institutionenspezifischen Funktionen dargestellt, die sich aus den Organisationszielen und -strukturen der Bundesagentur für Arbeit, der SGB-II- und Maßnahmenträger ergibt (Kapitel 5.5.1). Anschließend werden 2.) Typen der Aktivierung rekonstruiert, um die je spezifische Aktivierungsform der Maßnahmen Sozialer Aktivierung abzubilden. Mit diesen Typen gehen jeweils besondere soziale Integrationsformen einher. Somit erfüllen Maßnahmen Sozialer Aktivierung auch unterschiedliche soziale Funktionen (Kapitel 5.5.2). Diese Funktionen können einerseits alltagsstabilisierend, persönlichkeits- oder sozialkompetenz- 
fördernd gestaltet sein. Sie können jedoch auch sehr niederschwellig Arbeitstugenden und -strukturen sowie den Kontakt zur Arbeitsverwaltung für eine sehr arbeitsmarktferne Klientel befördern. Diese Funktionen müssen sich nicht mit den Leitlinien des Aktivierungsdiskurses und den Vorgaben der Bundesagentur für Arbeit oder den Funktionen für die Maßnahmenteilnehmenden decken, denn die Beschaffung erfolgt in Kontexten, die mehrere Interpretations- und Handlungsspielräume eröffnen. Daher werden in diesem Kapitel die Funktionstypen untereinander verglichen und auf den Aktivierungsdiskurs rückbezogen. In toto wird der Aktivierungsbegriff um die soziale Aktivierung erweitert, die Problemlagen einer sehr arbeitsmarktfernen Klientel wahrnimmt und diesen über Unterstützungsangebote entgegenwirken will. Maßnahmen Sozialer Aktivierung werden dabei auch selbst als Integrationselement wahrgenommen. So nehmen die Maßnahmen insgesamt übergeordnete sozialstaatliche Funktionen ein, die unter den Begriffen Ganzheitlichkeit, Förderung der Beschäftigungsfähigkeit und Dekommodifizierung gefasst werden (Kapitel 5.5.3).

\subsubsection{Institutionenspezifische Funktionen}

\subsubsection{Funktionen für die Bundesagentur für Arbeit}

Für die Bundesagentur für Arbeit haben Maßnahmen Sozialer Aktivierung insbesondere eine Clearing-Funktion, denn hier kann eruiert werden, ob Personen, die aktuell in der Arbeitsverwaltung betreut werden, wirklich erwerbsfähig sind oder in anderen Rechtsbereichen stehen müssten. Weiter ist insbesondere durch die Etablierung des Instruments Aktivcenter die sichtbare Möglichkeit gegeben, eine sehr arbeitsmarktferne Klientel zu aktivieren und die Beschäftigungsfähigkeit zu fördern, statt sie im, durch den Aktivierungsdiskurs negativ konnotierten, Passivleistungsbezug zu belassen. Über Maßnahmenketten oder zeitlich umfangreichere Maßnahmen entstehe eine Perspektive auf eine Erwerbstätigkeitsaufnahme für diese Klientel. Eine auch durch schrittweise und letztlich strikte Sanktionen nicht erreichbare Zielgruppe kann durch Maßnahmen Sozialer Aktivierung erreicht und zur Maßnahmenteilnahme motiviert werden. Dabei wird speziell arbeitsmarktfernen Jugendlichen in Maßnahmen, die Sport- und Kreativangebote mit Bildungsanteilen kombinieren, eine besonders hohe Motivation und eine höhere Erwerbsintegrationsquote zugeschrieben.

\subsubsection{Funktionen für den SGB-II-Träger}

Die Clearing-Funktion der Maßnahmen Sozialer Aktivierung ist ebenfalls für die SGB-II-Träger von Bedeutung: Denn Maßnahmenträger-Fachkräfte nehmen sich hier intensiv einer schwer erreichbaren Klientel an und klären, ob die Maßnah- 
menteilnehmenden überhaupt dem Rechtskreis des SGB II zugehören oder ob sie eine Therapie, Rehabilitation oder Verrentung anstreben sollten. Damit scheiden sie kurz- oder langfristig aus der zu aktivierenden Klientel aus. Auch wenn kurzfristig keine hohen Erwerbsintegrationen (im Sinne des Controlling) durch Maßnahmen Sozialer Aktivierung erzielt werden, so wird doch eine Aktivierung über Kontaktaufnahmen erfasst sowie Kennzahlen für Maßnahmenabbrüche respektive Absenzen vermindert. Darüber hinaus wird insbesondere von den Fachkräften, die direkt mit den SGB-II-Leistungsempfangenden in Kontakt stehen, vorgebracht, dass durch Maßnahmen Sozialer Aktivierung für eine sehr arbeitsmarktferne Klientel das langfristige Ziel einer Erwerbstätigkeit erreichbar wird, indem die Beschäftigungsfähigkeit gefördert wird. Dafür sollten jedoch stärker an der arbeitsmarktfernen Klientel orientierte Organisationsstrukturen aufgebaut werden, wie beispielweise die Bündelung mehrerer Angebote um eine Zielgruppe unter dem Dach eines SGB-II-Trägers. In Regio06 können zum Beispiel von Wohnungslosen die Themen Wohnen, Betreuung, SGB-II-Leistungen und Wirtschaftliche Hilfen (SGB XII) in einem Haus geklärt werden, da dort verschiedene Institutionen vertreten sind. Die durch die Wohnungslosigkeit schwer zu kontaktierende Klientel kann so beim ersten Behördengang die weiteren Schritte der Leistungsbeantragung und Förderung gemeinsam erledigen. Wenn die zuständigen Behörden nicht in einem Haus versammelt sind, können Maßnahmen Sozialer Aktivierung vielfach die Funktion erfüllen, ein soziales Netzwerk um die Teilnehmenden aufzubauen und die Bearbeitung von Problemlagen zu beginnen. So sollen häufig die Maßnahmenträger eine Vernetzung der sozialen und gesundheitlichen Integrationshilfen gewährleisten (Jobcenter, Gesundheits- und Jugendamt, Krankenversicherung, Schuldnerberatung etc.) und auf eine psychosoziale Stabilisierung der Maßnahmenteilnehmenden hinwirken. Häufig fungiert der Maßnahmenträger hier sogar nicht nur als Netzwerkelement, sondern als Mittler zwischen den Institutionen: Vorausgesetzt, es gelingt einem Maßnahmenträger Drittmittel einzuwerben, können Angebote, die nicht im Aufgabenbereich des SGB II liegen, jedoch die SGB-II-Klientel betreffen, mitfinanziert werden. Dies ermöglicht eine Kooperation von verschiedenen Institutionen rund um die arbeitsmarktferne SGB-II-Klientel, in der der Maßnahmenträger die Kooperation organisiert und mitgestaltet. Die SGB-II-Träger selbst sind derzeit überwiegend nicht darauf ausgerichtet, solche Kooperationsstrukturen in Eigenregie aufzubauen und vorzuhalten. Desgleichen ist es für die VertreterInnen der SGB-II-Träger deutlich schwieriger, eine Vertrauens- und Kommunikationsbasis herzustellen, als für die des Maßnahmenträgers. So nehmen Maßnahmenträger hier eine Mittlerrolle zwischen dem SGB-II-Träger und dem SGB-II-Leistungsempfangenden ein. 
»ARGE wird in erster Linie als Druck empfunden, ARGE ist diejenigen, die sagen, die machen mir Auflagen, da habe ich Druck, da muss ich das und das machen und mit der Haltung gehen natürlich auch die Klienten zur ARGE und wenn nicht nur dieses Fordern im Vordergrund stehen würde, sondern diese Förderkomponente auch spürbarer wäre, ich könnte mir schon vorstellen, aber das ist ein längerer Prozess. Also dieses Image wieder abzubauen ist sicherlich nicht einfach und das geht nicht in zwei Wochen." (Leiterin eines Sozialpsychiatrischen Dienstes, Interview05Regio02)

So dienen Maßnahmen Sozialer Aktivierung auch der Herstellung einer Kommunikationsbasis für sensible Themen und für schwer erreichbare Gruppen im SGB-II-Träger. Damit kann zum Teil erst die arbeitsverwaltende Bearbeitung einer zuvor bei Vorladung nicht erschienen Klientel erreicht werden. Weiter führen Maßnahmen Sozialer Aktivierung erweiterte Lernformen ein, etwa über sportliche Betätigung. Manche Maßnahmenträger machen die in Sporteinheiten erlernten sozialen und personalen Kompetenzen für einen Eintritt in das Erwerbsleben verantwortlich. Hier werden nicht nur auf körperlichen und geistigen Ebenen Lernziele gesetzt, sondern auch Frontalvorträge durch Lernen in Gruppenkonzepten und zum Teil aus der Peer-Gruppe heraus ergänzt oder sogar ersetzt. Letztlich haben die Maßnahmen damit einen auf mentaler und körperlicher Ebene disziplinierenden Charakter. Daneben können Maßnahmen Sozialer Aktivierung als Durchführung einer Gegenleistung für die SGB-II-Leistungen dienen, die im Aktivierungsdiskurs als notwendig hervorgehoben wird. Teilnehmende, die sich in solchen Maßnahmen im Sinne der Arbeitsverwaltung einbringen, können dann auch in der institutionsinternen Personenklassifikation aufsteigen. Zudem verbinden viele Fachkräfte aus der täglichen Interaktion mit den sehr arbeitsmarktfernen SGB-II-Leistungsbeziehenden auch ein Unterstützungsanliegen. Maßnahmen Sozialer Aktivierung sind dabei ein geeignetes Instrument, um Personen Unterstützungsangebote zu unterbreiten, die ihre Lebenssituation ändern möchten, sich jedoch nicht selbst helfen können. Insofern sind Maßnahmen Sozialer Aktivierung auch sozialpolitisch als Chance für und Schnittstelle zu Personen zu sehen, die von anderen sozialstaatlichen Institutionen nicht erreicht werden. Durch die finanzielle Abhängigkeit kommen in die SGB-II-Träger auch Menschen, die Hilfe benötigen würden und zum Beispiel nicht freiwillig den Sozialpsychiatrischen Dienst aufsuchen:

»und zur ARGE kommen alle, also das ist so das, wo ich so denke, also im Grunde genommen ist das die Stelle, die unheimlich viel mitkriegt, also die kriegen Klienten zu sehen, die wir nie zu Gesicht bekommen, weil die zu uns nämlich gar nicht erst kommen, 
weil die überhaupt nicht auf die Idee kommen, dass sie da vielleicht auch einmal hingehen sollten.« (Leiterin eines Sozialpsychiatrischen Dienstes, Interview05Regio02)

\subsubsection{Funktionen für den Maßnahmenträger}

Beschäftigungsförderungen dienen neben der Arbeitsmarkt- und Sozialintegration häufig auch dem Erwerb von Eigenmitteln für die Maßnahmenträger (Sowa et al. 2012, Bode 2011). Maßnahmen Sozialer Aktivierung werden über eine große Bandbreite von Finanzierungsoptionen umgesetzt. ${ }^{60}$ Für Maßnahmenträger ist hier einerseits relevant, dass zusätzliche Mittelgeber zu den regionalen Jobcentern hinzutreten können. Zum Beispiel geben der Europäische Sozialfond oder das Bundesministerium für Arbeit und Soziales Mittel aus dem Pakt 50plus. Die Ausschreibungen unterschiedlicher Institutionen erhöhen die Bandbreite möglicher Maßnahmen, um die einzelne Maßnahmenträger konkurrieren. Daneben kann die Verbindung aus mehreren Drittmittelprojekten auch eine Vielfalt des Angebots herstellen, die für die SGB-II-Träger und ihre Klientel relevant ist. Ein Beispiel wäre hier das sozialarbeiterische Rahmenprogramm der kurzfristigen Wohnungslosenunterkunft und der Bildungs- sowie Ferienreisen in dem christlichen Maßnahmenträger in Regio04, das nicht durch den Eingliederungstitel des SGB II oder sonstige Beschäftigungsförderungsprogramme finanziert werden könnte. ${ }^{61}$ Solche Angebote gehören nicht zu dem eigentlichen Portfolio des SGB II, sind jedoch für die Betreuung der sehr arbeitsmarktfernen Klientel relevant, da die Betreuungsleistung umfassender gestaltet werden kann. Wenn diese Angebotsvielfalt den Fachkräften des SGB-II-Trägers bekannt ist, wird ein solch vielseitiger Maßnahmenträger attraktiver, in der Vergabefrage weiterer Maßnahmen Sozialer Aktivierung. Auch die von Maßnahmenträger-Fachkräften diagnostizierte motivationssteigernde Wirkung für die Maßnahmenteilnehmenden ist nicht unerheblich für den Maßnahmenträger, denn geringere Abbruchquoten und mehr erfolgreich abgeschlossene Maßnahmenteilnahmen bringen eine höhere Bewertung im Controlling. Darüber hinaus sind auch die internen Verwaltungsabläufe entlastet, wenn die zugewiesenen Personen durchgehend an der Maßnahme teilnehmen. Hinzu tritt, dass einmal genutzte Ressourcen und Fachkräfte gerne weiter eingesetzt werden, um eine genügende Auslastung zu generieren. Ein Beispiel wären hier die Räumlichkeiten und Ressourcen für

60 Vergleiche Kapitel 5.2.3 Rechtliche Realisierungsform und lokale Umsetzungen der Maßnahmen Sozialer Aktivierung.

61 Vergleiche Kapitel 5.1.3 Fallbeispiel einer Maßnahme zum Heranführen an geordnete Familienstrukturen und -prozesse. 
Sportangebote eines Bildungsträgers in Regio02, ${ }^{62}$ der im Rahmen der Beantragung weiterer Maßnahmen, die Elemente einer laufenden Maßnahme Sozialer Aktivierung mit einbezog, um diese weiter auszubauen respektive fortführen $\mathrm{zu}$ können. Auch führt der den Maßnahmen Sozialer Aktivierung inhärente Ansatz, hin zu einer mittel- bis langfristigen Perspektive bei der Erwerbsintegration da$\mathrm{zu}$, dass Maßnahmenträger so selbst langfristiger planen, Ressourcen nutzen und Personal beschäftigen können. Diese betriebswirtschaftlichen, aber teilweise auch sozialpädagogischen Effekte zeichnen sowohl Maßnahmen Sozialer Aktivierung, als auch alle mittel- bis langfristigen Maßnahmen aus. Daneben kann die Aufnahme von Maßnahmen Sozialer Aktivierung in das Portfolio der Maßnahmenträger eine Nischenposition bieten, in der sie Konkurrenzvorteile erwerben. Allerdings äußern Maßnahmenträger-Fachkräfte durchaus auch ein Interesse, an der intensiveren Unterstützung einer arbeitsmarktfernen Klientel und einer Orientierung in diesen Maßnahmen an der Vermittlung von Grundkompetenzen, um Personen weiter zu fördern, die in anderen Maßnahmen bereits ausgeschieden wären. Ferner ist zu berücksichtigen, dass Maßnahmenträger aus bisweilen sehr unterschiedlichen Organisationskulturen entstanden. Variierende Organisationskulturen, etwa von rein gewerblichen Bildungsträgern, kommunalen Maßnahmenträgern oder Maßnahmenträgern in klassischen Sozialorganisationen finden sich nicht nur im organisationalen Selbstverständnis wieder. So bietet die Erhebung ein Bild, in dem sich zahlreiche Maßnahmenträger gezielt aus ihrem Selbstverständnis heraus den Maßnahmen Sozialer Aktivierung öffnen, um benachteiligte Personen unterstützen zu können. Bode betont die Verknüpfung der unterschiedlichen, ebenfalls in der vorliegenden Studie anzutreffenden, innerorganisationalen Motive: »Von ihrem Selbstverständnis her zielen die Projekte vieler Träger darauf ab, verschiedene Zwecke (ökonomische Betätigung, lokale Teilhabe, Engagement für das Gemeinwesen etc.) zu verknüpfen und dadurch auch die Sozialintegration der Maßnahmenteilnehmer zu fördern (vgl. Vomberg 2005; Bode et al. 2005).« (Bode 2011: 324)

Insgesamt zeigen sich für die Bundesagentur für Arbeit, die SGB-II-Träger und die Maßnahmenträger vielfältige Funktionen der Maßnahmen Sozialer Aktivierung auf institutioneller Ebene. Im Folgenden werden, über die Rekonstruktion der Typen der Aktivierung, die sozialen Funktionen aufgezeigt.

62 Vergleiche Kapitel 5.1.1 Fallbeispiel einer sozialarbeiterischen U25-Maßnahme in sozialem Brennpunkt. 


\subsubsection{Typen der Aktivierung: Soziale Funktionen}

Um die Frage zu klären, welche Funktionen die Maßnahmen Sozialer Aktivierung innerhalb des Aktivierungsdiskurses einnehmen, wird das Praxisfeld in Bezug zur herausgearbeiteten Aktivierungsdiskursformation ${ }^{63}$ gesetzt: Durch einen systematischen Vergleich zwischen den im qualitativen Material enthaltenen Deutungsmustern und den Analysekategorien wird eine methodische Restrukturierung vorgenommen - in Typen der Aktivierung, die den Aktivierungsdiskurs in je spezifischer Weise ausführen. Um die Maßnahmenfunktionen zu verstehen, wird ein typenbildendes Verfahren aus Sicht der SGB-II- und Maßnahmenträger $^{64}$ mit einer analytischen Konzentration auf spezifische Funktionen orientiert an Udo Kelle und Susann Kluge (Kelle/Kluge 2010b) vorgenommen. Die Kategorisierung basiert auf folgenden theoretischen Annahmen: ${ }^{65}$

Ein Sozialstaat ermöglicht seiner Bürgerschaft über politische und soziale Rechte (Marshall 1992) eine Integration in Gesellschaft durch soziale und berufliche Teilhabe (Kaufmann 2003). Da sich soziale Teilhabe in der Arbeitsgesellschaft (Opielka 2004) überwiegend über das Erwerbsprinzip vollzieht, ist die arbeitsmarktpolitische Gestaltung der Beschäftigungsförderung und damit die Ausgestaltung der Integrationsform von Bedeutung. Der Aktivierungsdiskurs zeichnet sich hier durch eine Verlagerung auf rekommodifizierende Instrumente aus, durch die Arbeitskraft rekapitalisiert und einer höheren Marktabhängigkeit unterzogen wird (Dingeldey 2007, 2010). Die Arbeitsverwaltung ist Teil des Regierungshandelns und kann einerseits strategische Machtbeziehungen und Herrschaftszustände offenlegen, vermittelt jedoch auch zwischen Macht und Subjektivität (Lemke et al. 2000: 8). Da die bürokratische Verwaltung Herrschaft kraft Wissen legitimiert, ist das Dienstwissen der Fachkräfte in der Arbeitsverwaltung von Bedeutung (Weber 2005). Ebenso die Alltagspraxis der Arbeitsverwaltung in Bezug auf die Ausgestaltung der Normen und Vorgaben des Aktivierungsdiskurses. Denn in dem Moment, da die Aktivierungspolitik auf eine arbeitsmarktferne Klientel stößt, die sich weder eigeninitiativ noch institutionell diszipliniert in Erwerb integriert (oder integrieren kann), entstehen Widersprüche, die neu in den Aktivierungsdiskurs und die Arbeitsverwaltung integriert werden müssen. Gleichzeitig treffen in der Arbeitsverwaltung politische Steuerung, sozialstaatli-

63 Vergleiche Kapitel 1 Aktivierungsdiskurs: Wandel der Arbeitsmarktpolitik.

64 Die hier vorliegende Typologie basiert auf dem Vergleich und der Kontrastierung der 45 in Deutschland erhobenen qualitativen Experteninterviews.

65 Vergleiche Kapitel 3.3 Forschungsleitende Annahmen. 
che Institutionen und Subjekte in einem Kontext von Politik, Recht und Ökonomie aufeinander, die unterschiedliche Interpretationen und Gestaltungsräume ermöglichen - Subjekte sind auch SprecherInnen des Diskurses. Daraus folgt, dass die Sicht auf das zu aktivierende Subjekt entscheidend ist, da sich im Prozess der Tätigkeiten der Arbeitsverwaltung Subjektivierungen vollziehen - über die Unterwerfung unter ein allgemeines Gesetz und spezifische Normen, gewinnt das Individuum Autonomie (Butler 2001).

Um die Funktionen zu beschreiben, wurden folgende Kategorien untersucht:

1.) Das Maßnahmenziel beschreibt, welche Maßnahmeninhalte mit welchen Intentionen verbunden sind.

2.) Die Sicht der Akteurinnen und Akteure der Arbeitsverwaltung beinhaltet, mit welchen Deutungen die Fachkräfte in SGB-II- und Maßnahmenträgern ihrer Klientel im SGB-II-Leistungsbezug begegnen und verweist somit auf die im Prozess der Arbeitsverwaltung stattfindende Subjektivierung.

3.) Die Elemente des Förderns und Forderns zeigen die Ausgestaltung der allgemeinen Zieldimension des Aktivierungsdiskurses in der jeweiligen Maßnahme auf. Damit werden Aspekte des Enablings und der Rekommodifizierung auf die Maßnahmenebene bezogen.

4.) Die Integrationsform beschreibt, welche Möglichkeiten der Teilhabe durch Beschäftigungen in Tätigkeiten, des Aufbaus von sozialen Beziehungen und Interaktionsformen, Strukturierungen des Alltags etc. bestehen. Da im bisherigen Verlauf der Arbeitsverwaltung eine Integration der Teilnehmenden der Maßnahmen Sozialer Aktivierung in Erwerbstätigkeit nicht gelang, werden die Maßnahmen bereitgestellt. Mit diesem Maßnahmenangebot fördert die Arbeitsverwaltung, nolens volens, bereits die Teilhabeoptionen, die dann in den einzelnen Maßnahmen recht unterschiedlich gewichtet werden.

5.) Die Aktivierungsform beschreibt die Umsetzung beziehungsweise Anwendung des Aktivierungsparadigmas als Reaktion der lokalen AkteurInnen der Arbeitsverwaltung auf Widersprüche, die bei bislang erfolgloser Erwerbsintegration von Leistungsbeziehenden entstehen. Da die Fachkräfte der SGBII- und Maßnahmenträger Deutungs- und Handlungsspielräume in der Maßnahmengestaltung haben, können sie diese Deutungsmacht nutzen und die Aktivierungsvorgaben des Aktivierungsdiskurses in der Praxis erweitern.

Die Kategorien wurden aus den oben dargestellten Vorannahmen als theoretischem Dach generiert und im Rahmen dieser Bezugspunkte komparativ verglichen bis eine größtmögliche Homogenität auf der Ebene der Typen erreicht wur- 
de (Kelle/Kluge 2010a). Zwar lassen sich im Feld bisweilen Maßnahmen mit der Zentrierung auf einzelne Maßnahmenfunktionen finden, doch meistens handelt es sich um Kombinationen mehrerer Funktionen, die in der Typologie systematisch und daher singulär gefasst wurden. So können einzelne untersuchte Maßnahmen mehrere Funktionen erfüllen, weisen jedoch eine analytisch trennscharfe Schwerpunktsetzung auf. Mit den im Folgenden dargestellten fünf Typen werden über schematische Maßnahmenstrukturen, aber auch mittels der dahinterliegenden Deutungsmuster von Aktivierung und Integration die Funktionen im Akteursfeld von SGB-II- und Maßnahmenträgern offengelegt. Während der Aktivierungsdiskurs vordergründig auf die Integration in Erwerbstätigkeit fokussiert, diffundieren die Funktionen in der Praxis in spezifische Teilschritte, die Integration in die Ordnung: 1.) der Tätigkeit, 2.) einer erwerbstätigen Familie, 3.) der Arbeitsverwaltung, 4.) des gesellschaftlichen Miteinanders und 5.) des aktiven Subjekts.

\section{Abbildung 8: Typen der Funktionen von Maßnahmen Sozialer Aktivierung}

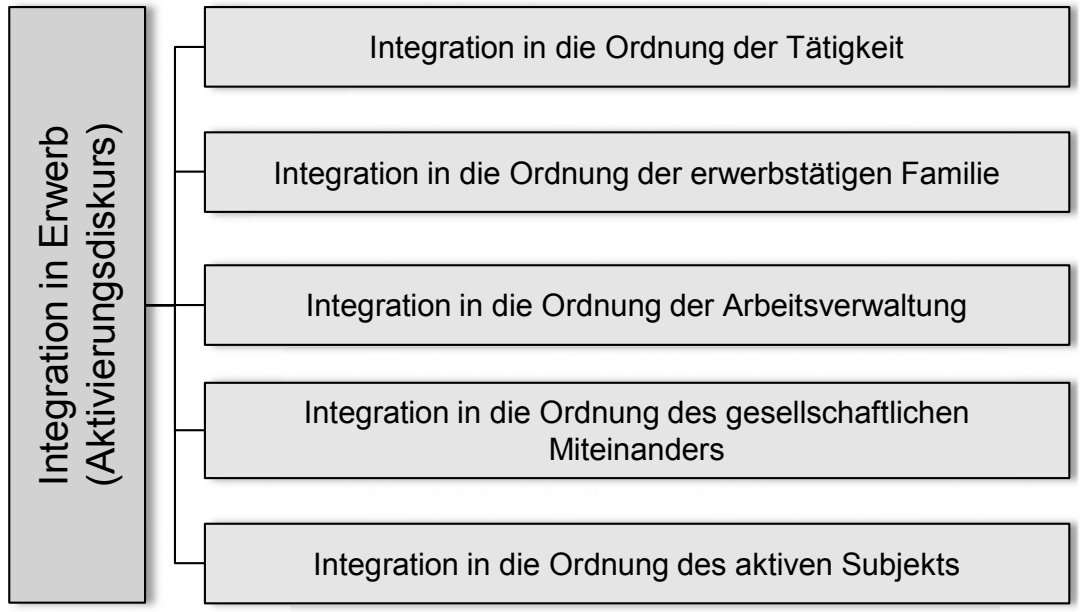

Quelle: Eigene Darstellung

Der Begriff Ordnung soll an dieser Stelle für normative Strukturen stehen, die im Praxisfeld als Ausführungsort des Aktivierungsdiskurses vorzufinden sind. In diese normativen Strukturen sind einerseits legitime Wissensformen eingebettet, die »zu Netzen angeordnet und durch rationale Schemata erfasst werden können « (Foucault 1974: 644). Andererseits sind auch AkteurInnen und Institutionen wie die Arbeitsverwaltung, die Sozialwirtschaft oder Schulden- und Suchtberatung integriert. Die Ordnungen, in dem oben dargestellten Sinne, wirken 
sich auf die Alltagsstrukturen der Maßnahmenteilnehmenden aus, indem Tagesabläufe simuliert und Voraussetzungen vorbereitet werden, die auf dem Arbeitsmarkt notwendig sind. Denn in einer langfristigen Perspektive intendiert auch die Integration in die fünf Ordnungen eine Erwerbsintegration. Doch die Typen stehen in ihrer unmittelbaren Ausrichtung konträr zum Aktivierungsdiskurs, da keine kurzfristige Erwerbsintegration, die im Sinne des Controllings messbar wäre, erfolgen kann. Es wird jedoch eine erweiterte Aktivierungsform gebildet, die eine teilweise Umdeutung des Diskurses ausdrückt. Denn selbst wenn die Erwerbsintegration nicht gelingt, kann doch eine erfolgreiche Aktivierung verzeichnet werden. Die in diesem Kapitel dargelegten Funktionen verweisen insbesondere auf die sozial aktivierenden Elemente in den Maßnahmen. Die weiteren häufig in den Maßnahmen der Beschäftigungsförderung enthaltenen Elemente werden in diesem Rahmen nicht explizit berücksichtigt.

\subsubsection{Typ Ordnung der Tätigkeit}

\section{Maßnahmenziel}

Dieser Typ der Maßnahmen Sozialer Aktivierung ist durch seinen Fokus auf die Tätigkeit den klassischen Instrumenten der Arbeitsmarktdienstleistungen am nächsten. Der Typ Tätigkeit ist primär dadurch charakterisiert, dass in den normativen Strukturen des Praxisfeldes Tugenden der Arbeit gefördert werden sollen. Unter diesen Arbeitstugenden wird hauptsächlich Folgendes (in nicht hierarchischer Reihenfolge) verstanden: Pünktlichkeit, Sauberkeit, Verlässlichkeit, Geduld, Sorgfalt, Mitdenken während des Arbeitsprozesses, geistige, körperliche und räumliche Mobilität und die Interaktion im Kontext einer arbeitsähnlichen Umgebung, wie situationsangemessenes Verhalten in hierarchischen Strukturen. ${ }^{66}$ Dabei wird auch der Aufbau eines geregelten Tagesablaufs als Voraussetzung angesehen, um eine Ausbildung oder Erwerbstätigkeit durchführen zu können. Über die Tätigkeit in der Maßnahme selbst sollen die Wirkungen der Intervention erreicht werden. Durchgeführt werden hier jedoch überwiegend nicht arbeitsmarktnahe oder ausbildungsinadäquate Tätigkeiten. Insgesamt zeichnet sich der Typ durch das Lernen über körperliche Erfahrungen aus: Hier »muss man viel, viel mit [physischer; $\mathrm{CF}$ ] Arbeit machen. Oder heute habe ich das fertig gekriegt, ich hab einen Erfolg.« (Teamleiterin, Interview04Regio02) So ist bei die-

66 In einem Maßnahmenträger der Regio03 wird berichtet, dass es zum Lehrinhalt gehört, vor dem Eintreten zu klopfen, zu grüßen und respektvoll miteinander umzugehen (Interview03Regio10). 
sem Typus eine häufig auftretende Rechtsform des Maßnahmeneinsatzes die Arbeitsgelegenheit nach $\S 16 \mathrm{~d}$ SGB II.

\section{Sicht der Akteurinnen und Akteure}

Die Klientel wird auf Basis fehlender Arbeitstugenden und -strukturen als weit vom Arbeitsmarkt entfernt beschrieben. In der Altersstruktur dominieren Jugendliche und über 50-Jährige. Dargestellt werden Individuen mit einer Ferne zu Theorie und schulischen Lernformen, schlechten Schulabschlüssen, niedriger Frustrationstoleranz und fehlenden Arbeitsstrukturen aufgrund (langjährig) fehlender Arbeitserfahrung. Bei über 50-Jährigen müssten insbesondere Frustrationen auf Basis zahlreicher erfolgloser Bewerbungen abgebaut werden. Die unter 25-Jährigen werden als die »ganz ganz komplizierten Jugendlichen« (Bereichsleiter, Interview01Regio04) beschrieben, bei denen es auch darum gehe, sie aus der peer-group herauszunehmen: »Einfach hier mal raus zu reißen aus der Clique, was ja gerade in solchen Ballungsgebieten ein großes Problem ist. Das ist nicht der einzige [im Sinne von: Es sind nicht die einzelnen; $\mathrm{CF}$ ] Personen, sondern die Gruppendynamik." (Bereichsleiter, Interview01Regio04). Denn in einem entsprechenden Umfeld würden die Individuen ihre fehlenden Arbeitstugenden legitimieren und damit perpetuieren. Daneben zeichne die Jugendlichen eine unrealistische Berufsorientierung aus. Genauer, es gibt auf dem Arbeitsmarkt keine Nachfrage nach ihren Wunschberufen oder die Jugendlichen verfügen laut Zuschreibung nicht über die notwendigen Fähigkeiten. Zudem belaste eine gänzlich fehlende oder über lange Jahre zurückliegende Arbeitserfahrung (insbesondere bei unter 25-Jährigen) die Tauglichkeit für den Arbeitsmarkt.

»Die [unter 25-Jährigen; CF] haben ja ganz oft schon vom Elternhaus her einfach überhaupt nicht gehört oder miterlebt, wie es ist, wenn man früh aufsteht und es geht jemand in die Arbeit und da versuchen wir, diese Formen aufzubrechen. Also mit ganz niederschwelligen Angeboten aber auch Arbeitsgelegenheit, weil der Jugendliche häufig die Schule satt hat, der war 8,9,10 Jahre in der Schule und der hat keinen Bock mehr sich in eine Maßnahme zu setzen, wo er wieder 5,6,7,8 Stunden nur bespaßt wird, sondern da ist unser Erfolg eigentlich der, dass die auch was arbeiten. Das sie also praktisch, auch mit den Händen etwas tun.« (Teamleiterin, Interview04Regio02)

In den Maßnahmen soll die Notwendigkeit der häufig im Feld genannten Arbeitstugenden vermittelt und erprobt werden. 


\section{Elemente des Förderns und Forderns}

Im Funktionstyp der Tätigkeit wird insofern eine Förderung vorgenommen, als Arbeitstugenden und -strukturen über die Tätigkeit (wieder-)erlernt werden sollen. Hier sollen Stärken durch die ausgeübte Tätigkeit sichtbar gemacht und die Maßnahmenteilnehmenden dazu motiviert werden, eine qualifizierende oder berufsvorbereitende Maßnahme anzuschließen sowie neue Berufs- oder Ausbildungsziele zu formulieren. Insbesondere die Erfahrung der körperlichen Tätigkeit soll zu einer Einheit von Geist und Körper führen. Das arbeitende Tätigsein wecke die jeweilige Motivation für eine arbeitsmarktliche Integration. Die Kehrseite der Medaille im Element des Forderns ist, dass die Arbeitstugenden und strukturen auch eingehalten werden müssen oder Sanktionen erfolgen. Denn die Erkenntnis der persönlichen Stärken und Interessen soll demnach zu einer Zielfokussierung führen, die die Richtung der weiteren Betreuung seitens der Arbeitsverwaltung auf einen spezifischen beruflichen Bereich konkretisiert. Ferner dient die Maßnahme der Arbeitserprobung und soll die Erreichbarkeit und Arbeitswilligkeit testen. Sanktionen erfolgen, wenn die Arbeitstugenden und strukturen fehlen beziehungsweise deren Bereitschaft zur Aneignung nicht gezeigt wird. Teilweise entstand bei der Erhebung und Analyse der Eindruck, als wenn Arbeitstugenden, die als Maßnahmenziel formuliert waren, bereits mit Beginn der Maßnahme vorausgesetzt wurden.

\section{Integrationsform}

Über weitgehend voraussetzungslose Arbeitstätigkeiten in den Bereichen wie Fahrradreparatur, Näharbeiten, Möbelaufbereitung, aber auch unterstützende Tätigkeiten in Holz- und Metallwerkstätten, Hausmeistertätigkeiten, Malerarbeit, Grünflächenarbeit, helfende Tätigkeiten auf einem Kinderbauernhof und Tierheim oder die Arbeit in Maßnahmen Sozialer Aktivierung soll soziale Teilhabe ermöglicht werden. ${ }^{67}$ Gegebenenfalls vollzieht sich in den Maßnahmen auch eine Berufsausbildung in den Branchen Küche und Hauswirtschaft. Die in den Werkstätten gefertigten Produkte (zum Beispiel Untersetzer, Regale, Dekorationen) können meist für den Eigenbedarf mit nach Hause genommen werden. Nähwerkstätten werden in den Maßnahmen dazu genutzt, zu lernen, wie die eigene Kleidung ausgebessert werden kann oder Spielzeuge für die Kinder der Teilnehmenden hergestellt werden können. Der Subsistenz-Gedanke findet sich auch im häuslichen Bereich: hier sollen kleinere handwerkliche Verrichtungen (zum

67 Vergleiche Interview01Regio04, Interview03Regio04, Interview04Regio04, Interview04Regio07, Interview07Regio13, Interview08Regio13. 
Beispiel Wandmontagen) erlernt werden. ${ }^{68}$ Die Tätigkeiten können auch Hilfebedürftigen, die nicht an der jeweiligen Maßnahme teilnehmen, zur Verfügung gestellt werden, wie etwa Fahrradreparaturen ${ }^{69}$ für andere SGB-II-Leistungsbeziehende. Auch die Produkte der sozial aktivierenden Küche können die Maßnahmenteilnehmenden meist selbst verzehren. ${ }^{70}$

\section{Aktivierungsform}

In diesem Typ liegt der Schwerpunkt der Ausgestaltung des Aktivierungsdiskuses bei Tätigkeiten für die Subsistenz, da die Produkte sowie die Erkenntnisse aus der Arbeit meist für den individuellen Gebrauch der Teilnehmenden von Bedeutung sind und nur in einer langfristigen Perspektive die hier vermittelten Fähigkeiten (nach einer weiteren Ausbildung, Schulung oder Arbeitserfahrung über Praktika) einen Erwerbseinstieg ermöglichen. Wenn in der Maßnahme bereits eine Ausbildung für helfende Tätigkeiten im Bereich der Hauswirtschaft oder der Küche stattfindet, lässt sich dies als außergewöhnlich starke Fokussierung auf den Arbeitsmarkt deuten. ${ }^{71}$

Zusammengefasst wird die Integration in die Ordnung der Tätigkeit über eine Teilnahme an unterstützenden und einfachen Aufgabenbereichen ermöglicht, die Dinge und Dienstleistungen für den Eigenbedarf erstellen. Aktiviert wird demnach zu einer Tätigkeit für die Subsistenz, um Tugenden der Arbeit zu erlernen und zu prüfen.

68 Vergleiche Interview04Regio04, Interview05Regio06, Interview04Regio10, Interview02Regio11.

69 Vergleiche Interview04Regio10.

70 Bei einigen Maßnahmenträgern versorgen die Küchen das gesamte Publikum eines Maßnahmenträgers zum Beispiel Mütter, die freiwillig und teilweise gegen Bezahlung Kurse im Familienorientierten Maßnahmenträger besuchen oder Personen, die handwerkliche Kurse im Sozialbetrieblichen Maßnahmenträger belegen.

71 Allerdings ist dieses Vorgehen nur in Regio04 und Regio07 zu finden. 
Tabelle 5: Typ Ordnung der Tätigkeit

\begin{tabular}{|l|l|}
\hline Kategorien/Typen & Ordnung der Tätigkeit \\
\hline Maßnahmenziel & Tugenden der Arbeit (wieder-)herstellen \\
\hline $\begin{array}{l}\text { Sicht der Arbeitsverwal- } \\
\text { tung auf die Maßnahmen- } \\
\text { teilnehmenden }\end{array}$ & $\begin{array}{l}\text { Individuen, mit Ferne zu Theorie und schuli- } \\
\text { schem Lernen, Frustration, fehlende grundle- } \\
\text { gende Arbeitsstrukturen aufgrund (langjährig) } \\
\text { fehlender Arbeitserfahrung }\end{array}$ \\
\hline Elemente des Förderns & $\begin{array}{l}\text { Arbeitsstrukturen herstellen über Tätigkeit, Auf- } \\
\text { zeigen von Motivationsressourcen, Lernen über } \\
\text { körperliche Erfahrung }\end{array}$ \\
\hline Elemente des Forderns & $\begin{array}{l}\text { Tugenden der Arbeit sollen angeeignet werden, } \\
\text { Überprüfung der Erreichbarkeit, Arbeitswillig- } \\
\text { keit und Arbeitserprobung }\end{array}$ \\
\hline Integrationsform & $\begin{array}{l}\text { Tätigkeiten, die für den Arbeitsmarkt mittelbar } \\
\text { und zum Teil direkt verwertbar sein können } \\
\text { (Fahrradwerkstatt, Holz- und Metallwerkstätten, } \\
\text { Maler-, Grünflächenarbeit etc.); Arbeitsgelegen- } \\
\text { heit in der Entgeltvariante }\end{array}$ \\
\hline Tätigkeit für Subsistenz \\
\hline
\end{tabular}

Quelle: Eigene Darstellung

\subsubsection{Typ Ordnung der erwerbstätigen Familie}

\section{Maßnahmenziel}

In Maßnahmen des Typs Ordnung der erwerbstätigen Familie ${ }^{72}$ (Bedarfsgemeinschaften mit ein bis drei Kindern bis 14 Jahren) sollen alle relevanten Institutionen (Jugendamt, Sozialdienste, Strafvollzug, ARGEN/Jobcenter etc.) ge-

72 Auch Maßnahmen für Alleinerziehende sind teilweise ähnlich ausgerichtet und sollen Bedingung für eine spätere Erwerbstätigkeit herstellen (Interview04Regio02, Interview02Regio11). 
meinsam mit den Familien ${ }^{73}$ Bedingungen schaffen, um zukünftig die Erwerbstätigkeit einer oder mehrerer Personen der Familie zu ermöglichen. Hemmnisse für einen Berufseinstieg sollen beseitigt werden. Die Vernetzung der Institutionen, die für diese Personen zuständig sind, fällt meist dem Maßnahmenträger zu. Hier sollen Hemmnisse übergreifend ermittelt und bearbeitet werden: »wir haben die Aufgabe, die Familie mal als Ganzes zu betrachten und zu versuchen, dass Probleme aus dem Weg geräumt werden, also die gesamte Familie zu aktivieren.« (Maßnahmenträgerleiterin, Interview01Regio02) Zu diesem Zweck wird beispielsweise in Regio02 die gesamte Kernfamilie (Eltern und Kinder) in den Träger eingeladen, um gemeinsam mit SozialpädagogInnen und einer Psychologin mögliche Schritte zu planen, um Sucht-, Schulden- oder sonstige Problemlagen bearbeiten zu können.

»Und das ist eine ganz tolle Sache und dort steht die Arbeit, erst an letzter Stelle und durch die Arbeit dann, soll versucht werden auch, die Leute dann langfristig in Arbeit zu bringen. Ne, so dass die Familie dann irgendwann mal weg kommt von der ARGE.« (Maßnahmenträgerleiterin, Interview01Regio02)

Die Maßnahme ist zunächst als Ein-Ernährer-Modell gedacht, wobei bei Interesse der zweiten erwachsenen Person im Haushalt eine Teilzeitbeschäftigung oder Maßnahme aufgenommen werden kann. Allerdings wird dies erst angestrebt, wenn das jüngste Kind älter als drei Jahre ist. Prägnant wird das Ziel der Maßnahmen diesen Typs von einer Maßnahmenträgerleiterin (Interview01Regio02) formuliert: Es geht um eine »Vorbereitung zur Arbeitsfähigkeit, nicht um die Arbeit selbst, also die ARGE hat auch dazugelernt.« (Maßnahmenträgerleiterin, Interview01Regio02)

\section{Sicht der Akteurinnen und Akteure}

Die Teilnehmenden werden als Menschen mit familiären, ${ }^{74}$ psychischen, physischen und/oder finanziellen Problemlagen von den AkteurInnen der Beschäfti-

73 Familie steht in dieser Perspektive für eine soziale Lebensform, die mindestens ein Kind und ein Elternteil umfasst und neben einem verwandtschaftlichen Verhältnis respektive Adoption durch Solidarität und persönliche Verbundenheit charakterisiert sein kann (Fuchs-Heinritz, 1995). Diese Definition sieht bewusst von biologischen oder politisch propagierten Dimensionen der Familie ab.

74 Da es häufig innerhäusliche Probleme in diesen Familien gebe, sei die Zusammenarbeit mit dem Jugendamt von großer Bedeutung, so die AkteurInnensicht. 
gungsförderung beschrieben. Zum Teil erstrecke sich die Erwerbslosigkeit in diesen Familien über mehrere Generationen - für viele befragte Personen eine Form negativer Tradierung. Argumente wie fehlende Kinderbetreuung, die dazu führe, dass etwa Schichtarbeit nicht angenommen werden könne, werden von SGB-II- und Maßnahmenträgerbeschäftigten häufig als Vorwand gedeutet. ${ }^{75}$ Durch die Unterstützung in der Organisation der notwendigen Infrastruktur, sollte die Klientel keine Hemmnisse der Erwerbsaufnahme mehr anführen können.

»Das lebt auch ein bisschen so davon, ja einfach, dass die nicht auskönnen, also ne? Es ist praktisch immer jemand da. [...] Diese ganzen Ausreden, die man vorher hatte, ja ich finde ja keinen Kindergartenplatz, ja nach der Schule habe ich ja keinen Hortplatz für mein Kind und solche Sachen, also das ist praktisch da erledigt.« (Teamleiterin, Interview04Regio02)

Angenommen wird, dass diese Klientel für die Notwendigkeit der Erwerbstätigkeit eines Elternteils sensibilisiert und motiviert werden muss:

»Das wir schauen, das die wirklich in der Zeit, in der sie in der Maßnahme sind für die Familie etwas tun, auch so dieses wecken dieses ich tue das für meine Kinder, ich tue das für meine Familie. Es ist nicht normal, dass Mama, Papa jahrelang daheim sitzen und den ganzen Tag Fernsehschauen, die Kinder im Kinderzimmer Fernsehschauen und das ist Familienleben.« (Teamleiterin, Interview04Regio02)

Es wird als notwendig erachtet, dass mindestens ein Elternteil erwerbstätig wird, um eine Vorbildfunktion zu erfüllen. Denn Kinder würden sonst nicht die notwendigen Alltagsstrukturen und Rahmenbedingungen für die spätere eigene Erwerbstätigkeit erlernen:

»Es soll eigentlich in die Richtung gehen [...] die Kinder sollen lernen, dass es normal ist, dass die Eltern arbeiten und die Eltern sollen auch lernen, dass es, dass ihr Alltag trotzdem strukturierbar ist, auch wenn man arbeiten geht und das eben in enger Zusammenarbeit

75 In einer Studie zu Dienstleistungsprozessen am Arbeitsmarkt hatten zwei Fünftel der SGB II-Leistungsbeziehenden neben den oben genannten Beschwernissen Kinderbetreuungs-, Ehe-/Beziehungs-, Sucht-, psychosoziale und sonstige soziale Probleme (sowie Pflegeverantwortung), teilweise auch mehrere gleichzeitig, wohingegen diese Problematiken in der Beratungspraxis wenig Raum einnehmen (Schütz et al. 2011: $314)$. 
mit dem Jugendamt und dem Allgemeinen Sozialdienst, dass wenn da Probleme auftreten egal in welcher Richtung, dass dann immer jemand da ist und fängt die auf. « (Teamleiterin, Interview04Regio02)

So wird angenommen, dass sich die Individuen andernfalls an die Arbeitslosigkeit »gewöhnen« könnten und sich über mehrere Generationen hinweg die Arbeitslosigkeit verfestige:

»Die muss ich aktivieren, dass die sich nicht gewöhnen an ihre Bedingungen. Dann haben wir ja auch hier das Problem der zweiten, dritten Generation die da reinrutschen. Also diese Prägung durch das soziale Umfeld ist sehr, also ist ein sehr großes Problem und da müssen wir die herausholen und deshalb sagen wir hier: möglichst viel aktivieren.« (Bereichsleiter, Interview01Regio04)

Die Aktivierung soll in langfristiger Perspektive $\mathrm{zu}$ einer Erwerbsintegration führen. Ähnliches konstatiert der Armuts- und Reichtumsbericht des Bundesministeriums für Arbeit und Soziales von 2013. Am stärksten von Armut betroffen sind die Kinder von Arbeitslosen. Demnach übernehme die Erwerbstätigkeit mindestens einer Person je Haushalt eine Schutzfunktion vor Armut (BMAS 2013b: 48). Spezifisch für die Sichtweise der Interviewten auf das KlientelSegment der Jugendlichen in diesen Familien ist, dass der Vorbildcharakter langzeitarbeitsloser Eltern für eine mangelnde Motivation der Kinder zur Erwerbsarbeitsaufnahme angeführt wird.

»Dass man eigentlich bei den Kindern anfangen muss, dass es nichts hilft, wenn der dann aus der Schule herauskommt, ist 18, 19 Jahre alt, da ist der Zug abgefahren, den motivierst du nicht mehr, auf Dauer ne. Das es ganz wichtig ist, grad so Kinder noch zu motivieren, eine Ausbildung zu machen, in die Arbeit zu gehen, zu zeigen wie das ist.« (Teamleiterin, Interview04Regio02)

\section{Elemente des Förderns und Forderns}

Die Förderung in der Maßnahme lässt sich als Bearbeitung von Hemmnissen interpretieren. Die Bereitstellung notwendiger Infrastruktur und die Persönlichkeits- sowie Familienförderung sollen langfristig zu einer Erwerbstätigkeit führen. Beispielsweise wird in Kooperation mit dem Jugendamt und Allgemeinen Sozialen Dienst in Regio02 eine Betreuung von Familien realisiert. Der Allgemeine Sozialdienst begleitet die Kinder zur Schule, SozialpädagogInnen sind über aufsuchende Dienste regelmäßig in der Familie präsent und auch der Kon- 
takt zum Jugendamt wird intensiviert. Gefördert werden die Familienstrukturen und sozialen Beziehungen dahingehend, dass Erwerbstätigkeit ermöglicht werden kann. Dies kann die Unterstützung durch SozialarbeiterInnen bei der Suche nach einer Kinderbetreuung sein, der Bearbeitung von Suchtproblematiken oder Schulden. Von Seiten der Arbeitsverwaltung wird die Teilnahme an einer Arbeitsgelegenheit gefordert. Darüber hinaus Bewerbungsbemühungen im Rahmen einer intensiven bewerbungsorientierten Betreuung durch die SGB-II-Träger, um während der Maßnahmendauer eine Anschlussbeschäftigung zu finden. In der Maßnahme erhalten die sozialstaatlichen Einrichtungen auch einen sehr tiefgehenden Einblick in die Privatsphäre der Familien und der über die verstärkte Unterstützungsleistung laufende Druck zur Bearbeitung von Problemlagen wird deutlich spürbarer. Durch die Vernetzung der Institutionen über den Maßnahmenträger wird eine stärkere Gesamtschau der Hemmnisse und der unternommenen Bearbeitungsschritte für die Arbeitsverwaltung ermöglicht.

\section{Integrationsform}

Sind alle Vorbedingungen erfüllt, sollte mindestens eine Person im Rahmen einer AGH (Mehraufwands- oder Entgeltvariante) erste Tätigkeiten aufnehmen. Die soziale Teilhabe erfolgt über unterstützende Tätigkeiten, die in der Entgeltvariante in einer gehaltsähnlichen Form monatlich anerkannt werden:

»Wir versuchen das zu simulieren in der Maßnahme, Arbeit, und das ist so, dass ein Elternteil dann eine Arbeitsgelegenheit-Entgeltvariante-Maßnahme macht, also wo die ganz normal bezahlt werden auch, wo Lohn aufs Konto kommt und so versucht man dieses >Arbeit` zu simulieren.« (Teamleiterin, Interview04Regio02)

Die Tätigkeiten können dabei in den unterschiedlichsten Einsatzfeldern vorgenommen werden, die sich gegebenenfalls an unterstützenden Tätigkeiten in Berufen orientieren, die die Teilnehmenden erlernt haben. Die Arbeitsgelegenheiten sind arbeitsähnlich gestaltet, müssen jedoch zusätzlich und gemeinnützig sein, den allgemeinen Kriterien der AGH entsprechend.

\section{Aktivierungsform}

Der Aktivierungsdiskurs wird hier in der Form ausgeführt, dass in einer simulierten Erwerbstätigkeit aktiviert wird, da die Maßnahme mit arbeitsähnlichen Tätigkeiten sowie Randbedingungen auf eine Erwerbstätigkeit vorbereiten soll. 
Tabelle 6: Typ Ordnung der erwerbstätigen Familie

\begin{tabular}{|l|l|}
\hline Kategorien/Typen & Ordnung der erwerbstätigen Familie \\
\hline Maßnahmenziel & $\begin{array}{l}\text { Rahmenbedingungen für die Ermöglichung der } \\
\text { Berufstätigkeit einer oder mehr Personen in der } \\
\text { Familie (wieder-)herstellen }\end{array}$ \\
\hline $\begin{array}{l}\text { Sicht der Arbeitsverwal- } \\
\text { tung auf die Maßnahmen- } \\
\text { teilnehmenden }\end{array}$ & $\begin{array}{l}\text { Individuen, die »Ausreden« wie unvereinbare } \\
\text { Arbeitsanforderungen mit dem Familienleben } \\
\text { gegenüber dem SGB-II-Träger nennen }\end{array}$ \\
\hline Elemente des Förderns & $\begin{array}{l}\text { Begleitete Organisation von Bedingungen, die } \\
\text { eine Erwerbsaufnahme ermöglichen: z.B. Kin- } \\
\text { derbetreuung, Bearbeitung von o.g. Problemen }\end{array}$ \\
\hline Elemente des Forderns & $\begin{array}{l}\text { Offenheit und Unterstützung bei der intensiven } \\
\text { Begleitung von Dritten, um Problemlagen der } \\
\text { Familie zu erkennen und bearbeiten }\end{array}$ \\
\hline Integrationsform & $\begin{array}{l}\text { Tätigkeiten für einen Familienangehörigen im } \\
\text { Rahmen einer Arbeitsgelegenheit }\end{array}$ \\
\hline Aktivierungsform & Simulation von Erwerbstätigkeit \\
\hline
\end{tabular}

Quelle: Eigene Darstellung

Zusammengefasst werden in diesem Typus mindestens eine Person und ihr familiäres Umfeld auf die Erwerbsaufnahme direkt vorbereitet, indem über eine Arbeitsgelegenheit in der Mehraufwands- oder Entgeltvariante eine Erwerbstätigkeit simuliert wird. Mithilfe dieser Betreuung sollen tägliche Familienproblematiken sowie externe Hemmnisse bearbeitet werden, die dem Erwerbsleben entgegenstehen. Ziel der Maßnahme ist, dass im Anschluss eine sozialversicherungspflichtige Beschäftigung für mindestens ein Elternteil ermöglicht wird.

\subsubsection{Typ Ordnung der Arbeitsverwaltung}

\section{Maßnahmenziel}

Ziel der Maßnahmen Sozialer Aktivierung ist es in diesem Typ, die Teilnehmenden in die normativen Strukturen der Arbeitsverwaltung zu integrieren. Denn die 
hier visierte Zielgruppe ist für die Arbeitsverwaltung kaum zugänglich, ${ }^{76}$ erscheint auf Einladungen und selbst nach schrittweisen und zum Teil starken Sanktionen nicht zu Gesprächsterminen im SGB-II- oder Maßnahmenträger: »Die kommen auf unsere Einladung nicht. « (Markt- und Integrationsexpertin, Interview03Regio12) Doch gemäß der Aktivierungsnorm müssen SGB-II-Leistungsbeziehende in regelmäßigen Abständen kontaktiert werden und zum Stand der Bemühungen um Arbeit befragt respektive unterstützende Maßnahmen eingeleitet werden, die im Rahmen von Kennzahlen für das Controlling erfasst und eingehalten werden müssen. Konventionelle Qualifizierungsmaßnahmen könnten dieses Ziel allerdings nicht erreichen, weil »ich am Ende nicht weiß, ob die [Teilnehmenden; CF] da wirklich landen.« (Fallmanager, Interview02Regio10) So wäre es erforderlich, bestimmte Teilnehmende von zu Hause abzuholen und in ihre laufende Maßnahme zu bringen. Genau dies leisten Maßnahmen Sozialer Aktivierung in diesem Typ. Sie dienen ebenso zur Aufklärung von konkreten Vermittlungshemmnissen respektive der Offenlegung, warum Personen nicht zu Gesprächsterminen in den SGB-II-Trägern erscheinen:

»Bis ich dann auch mal jemand [Maßnahmenträger; $\mathrm{CF}$ ] hatte, wo ich sag, besuch den doch mal zu Hause, was ist denn da passiert, der Briefkasten ist voll. Es ist gar kein Namensschild dran. Der wohnt da vielleicht gar nicht mehr. Das sind ja alles Ergebnisse, wo wir als Behörde einfach viel zu weit weg sind.«(Fallmanager, Interview02Regio10)

\section{Sicht der Akteurinnen und Akteure}

Die erst durch die Kontaktierung in Maßnahmen Sozialer Aktivierung zugänglichen Individuen werden als äußerst arbeitsmarktfern beschrieben. Sie weisen demzufolge starke soziale und physische Beeinträchtigungen auf: belastende soziale Hintergründe in Familien, traumatische Erfahrungen, Obdachlosigkeit, Suchttendenzen oder -krankheiten, Handicaps, geringe schulische und berufliche Qualifikation. Dabei seien die unter 25-Jährigen weit überrepräsentiert:

»Bei uns hat, bis auf wenigen Ausnahmen, niemand einen Schulabschluss [...] Weil sie wirklich mit so großen Schwierigkeiten und auch mit Lernbehinderungen oder psychischen Verstörtheiten [...] oder seelischen Behinderungen hierher kommen, sie kommen ei-

76 Die Nicht-Erreichbarkeit wird zum Beispiel thematisiert in Interview03Regio02, Interview02Regio05, Interview03Regio08, Interview03Regio11, Interview01Regio12 und Interview03Regio12. 
nem ja nicht vor. Man empfindet sie nicht so, als wären sie tatsächlich in diesem Alter 23, 24.« (Maßnahmenträgerleiterin, Interview03Regio04)

Eine Vielzahl wird als »Theoretisch richtig schwach, sozial, vom Verhalten her, ganz eher noch ziemlich verstört und in sich gekehrt.« (Maßnahmenträgerleiterin, Interview03Regio04) dargestellt.

»Wir haben den Kaffeesatz hier, die wirklich am sozial beeinträchtigtsten Jugendlichen sind hier bei uns, oder speziell jetzt in meiner Betreuung. Wo eben die Hintergründe mit Schulden, Drogen und Obdachlosigkeit. Also die ganze kriminelle Karriere da auch hinter steht [...]. Und die aufzufangen und wieder aktiv zu machen, ist natürlich auch sehr intensiv.« (Fallmanagerin, Interview02Regio04)

In der Gesamtheit der SGB-II-Leistungsempfangenden tritt diese Klientel quantitativ eher randständig in Erscheinung, doch wird die Auseinandersetzung mit diesen Individuen von AkteurInnen der Arbeitsverwaltung als eminent notwendig erachtet, da die Personen sonst unter Umständen unbemerkt im dauerhaften Sozialleistungsbezug stehen würden: »Sicher finden wir in den Ergebnissen der Statistik des Jobcenters nicht so den Stellenwert. Aber wir bereiten sie [die Teilnehmenden; CF] mit unseren Maßnahmen vor, weil ansonsten würden sie mit Sicherheit in irgendeinem Raster landen« (Fallmanagerin, Interview04Regio04).

\section{Elemente des Förderns und Forderns}

Die Aktivierungsnorm und die in den SGB-II- und Maßnahmenträgern Beschäftigten stoßen jedoch an ihre Grenzen, wenn die zu aktivierenden Personen trotz Sanktionen nicht erscheinen. Um der Aktivierungsnorm zu genügen, werden im Rahmen dieses Maßnahmentyps daher weitere Wege gesucht, um die nichterscheinenden Personen kontaktieren und aktivieren zu können. Elemente des Förderns und Forderns sind in diesem Rahmen 1.) aufsuchende Dienste, 2.) der direkte Besuch einer peer-group und 3.) Maßnahmen, die eine stärkere Attraktivität für die jeweiligen Zielgruppen aufweisen sollen.

1.) Die aufsuchenden Dienste ${ }^{77}$ können in Kooperation mit dem Allgemeinen Sozialdienst, doch auch über den Maßnahmenträger direkt durchgeführt werden.

77 Vergleiche Interview03Regio12, Interview01Regio04, Interview02Regio04, Interview03Regio04. 
Die Maßnahmenträger-Fachkräfte begeben sich zum Wohnort der zur Maßnahmenteilnahme verpflichteten Personen und suchen das Gespräch, wenn diese fernbleiben. »Mal bei ihm zu klingeln und zu sagen: wo liegen denn die Probleme? Warum kommst Du nicht? « (Markt- und Integrationsexpertin, Interview03Regio12) Diese Besuche werden als sehr effektives und »liebstes« (Sozialpädagogin, Interview03Regio04) Mittel beschrieben, die vorrangig Jugendliche zur Mitarbeit anregen sollen. Die Kontaktaufnahme werde eher genutzt als die reguläre Sanktionskette. Denn die Erfolge, definiert als das Erreichen der Jugendlichen, seien größer und erhöhten die Bereitschaft zur Maßnahmenteilnahme (Arbeitsvermittlerin, Interview02Regio04). Zugleich werden die Hausbesuche als Möglichkeit für die Maßnahmenträgerbeschäftigten gesehen, etwas über die Jugendlichen, deren soziales Umfeld und Lebensbedingungen zu erfahren. Durch die Rückmeldung der Maßnahmenträger zeige sich, dass es trotz der Möglichkeit aufsuchender Dienste schwer sei, die Klientel zu erreichen, doch:

»Wenn man den Kunden denn darüber gepackt hat, ist er dann in der Regel auch regelmäBiger da. Weil [...] die Träger auch nicht nur einmal, sondern auch ein zweites und ein drittes Mal und immer wieder nachhaken. Und irgendwann fühlen sich dann die Jugendlichen auch verstanden und also: da kümmert sich wirklich einer um mich. Die Problematik liegt ja meistens nicht zwingend am Jugendlichen selber, sondern auch durch das familiäre Umfeld und die sind das zum Teil einfach nicht mehr gewöhnt, dass man einmal sich so um sie kümmert und auch die Art und Weise wie man mit ihnen redet, was man versucht mit ihnen zu machen.« (Markt- und Integrationsexpertin, Interview03Regio12)

Das hier angeführte »Kümmern« hat neben dem fördernden Effekt eines Interesses an dem zu aktivierenden Individuum jedoch auch durch die Präsenz im unmittelbaren Lebensumfeld der SGB-II-Leistungsbeziehenden den fordernden Effekt einer stärkeren Vergegenwärtigung der disziplinarischen Handlungsmöglichkeiten der Beschäftigungsförderung.

2.) Auch werden zur Kontaktaufnahme mit einer spezifischen Personengruppe für den SGB-II-Bereich ungewöhnliche Wege beschritten. So suchte das Fallmanagement in Regio04 einen lokalen Treffpunkt von Punks auf, um mit der Klientel überhaupt in Kontakt zu treten:

»und haben gesagt: Na passt auf Jungs, Sozialhilfe ist nicht mehr - Hängematte - jetzt haben wir Arbeitslosengeld II und unter 25 doch etwas härtere Sanktionsmöglichkeiten. Wie können wir euch denn helfen, dass ihr irgendwann doch den Weg in Arbeit, Ausbildung, Schule wie auch immer findet? Wir sind alle obdachlos, wir haben alle Hunde und Arbeiten gehen, Hund an der U-Bahn lassen, geht nicht.« (Fallmanagerin, Interview04Regio04) 
Die Fallmanagerin sieht eine nicht zu unterschätzende Rolle der Hunde auf die psychische Befindlichkeit der Maßnahmenteilnehmenden, was sie anhand der guten Erziehung der Hunde und der Beobachtung illustriert, dass selbst wenn die Besitzer nichts zu essen haben, die Hunde noch stets gut versorgt sind. So wurde über die Rücksprache mit der zu aktivierend Klientel herausgefunden, dass als Maßnahmenrahmenbedingung eine Unterbringungsmöglichkeit für diese Tiere geschaffen werden müsse. Es wurde ein Maßnahmenträger gefunden, der mit Tierheimen kooperierte, so dass die Hunde während der Maßnahmenzeit betreut wurden. Daraufhin wurde »groß mit einem >Punker-Frühstückı eröffnet. [...] die waren so Feuer und Flamme dabei, das erste halbe Jahr lief ganz ganz toll. [...] Was wir nicht beachtet haben, dass ja alle eigentlich eine Suchtproblematik mitgebracht haben.« (Fallmanagerin, Interview04Regio04) So wurde im Maßnahmenverlauf zusätzlich mit einem Verein kooperiert, der Suchtkranke unterstützt. Der wechselseitige Austausch zwischen SGB-II-Leistungsbeziehenden und träger ließ eine Maßnahme entstehen, die einerseits die Förderung der Arbeitsmarktfähigkeit ermöglicht und andererseits die nun geschaffene Erreichbarkeit der Klientel und die Bearbeitung von Hemmnissen einfordern kann. Die Entdeckung der Suchtproblematiken und die flexible Reaktion durch eine weitere Kooperation veranschaulichen zwei wesentliche Elemente der Maßnahmen Sozialer Aktivierung: 1.) die gegenseitige Bedingung unterschiedlicher Arbeitsmarkthemmnisse und 2.) die flexibel-innovative Vorgehensweise der Maßnahmen in Planung und Durchführung.

3.) Wird über Teilnehmende ansprechende Angebote und kostenlose Essensausgaben versucht, die Personen zur Maßnahmenteilnahme zu bewegen. Über attraktive Angebote könne eine Kommunikationsbasis mit Personen gefunden werden, die sonst nicht für SGB-II- und Maßnahmenträger zugänglich sind:

»denn die Workshops müssen ja denn, die kitzeln ne, und das ist klar. Und das ist einmal der Sport, das kann die Musik sein, irgendwo zu komponieren, was zusammenzustellen, Musik-Gruppen zu bilden, irgendwie so in der Richtung, schneiden, was zusammen tun. Es muss den Jugendlichen ansprechen, ansonsten kriegen sie den nicht in die Hand.« (Maßnahmenträgerleiterin, Interview01Regio02)

Im christlichen Jugendträger existiert beispielsweise ein Bereich mit Kicker und Sitzgelegenheiten, der für alle Interessierten zugänglich ist und an einen Jugendclub erinnert: »Ohne Zuweisungskorridor, ohne Zwang, ohne Termin können die da einfach erscheinen und werden ebenso auch aufgefangen.« (Fallmanagerin, Interview02Regio04). Dieser Bereich fungiert als offene Anlaufstelle für Bedürf- 
tige des gesamten Wohnviertels. Wohnungslosen, unabhängig davon, ob dem SGB-II-Regelkreis zugehörig, werden täglich geöffnete Notunterkünfte $^{78}$ in Regio04 bereitgestellt:

»Wenn die [Jugendlichen im sozialen Brennpunkt; $\mathrm{CF}$ ] mal gekifft haben und völlig von der Rolle sind, können dort [in der Einrichtung des christlichen Jugendträgers; CF] auch mal ausnahmsweise übernachten. Also sie schaffen dort solche Möglichkeiten.« (Bereichsleiter, Interview01Regio04)

Frühstück, Mittagessen und Abendbrot, die von den Maßnahmenteilnehmenden im Küchen-Bereich vorbereitet werden, können gemeinsam eingenommen werden. Gefördert werde hier mittels offener Strukturen, einer offenen Kommunikation für die Interessen der SGB-II-Leistungsbeziehenden (zum Beispiel über Musik, Comics) und einer auf gegenseitigen Respekt ausgerichtete Interaktion. Mit einem Prinzip der permanenten Irritation, sollen die Teilnehmenden mit Situationen, Ansprachen, Haltungen und Handlungen konfrontiert werden, die sie aus ihrer Behördenerfahrung nicht kennen. Damit sollen eingefahrene Handlungsmuster sowie Vorstellungen auf Seiten der Teilnehmenden außer Kraft gesetzt werden, um sie zu motivieren. Gleichzeitig dienen die im offenen Kontext erfahrenen Informationen über die SGB-II-Leistungsbeziehenden jedoch auch der Personenanalyse für den SGB-II-Träger und bilden so eine allgegenwärtige Präsenz der Aktivierungsnorm. Auch wenn diese Angebote eigentlich ohne Verbindung zum Jobcenter stattfinden, kommuniziert zum Beispiel der christliche Jugendträger in Regio04 dem SGB-II-Träger, welche Jugendlichen wie oft anwesend sind. Exemplarisch wird hier das sprichwörtliche Vehikel des Beratungsbusses aus Regio04 vorgestellt: Der Beratungsbus als ein Angebot des christlichen Jugendträgers ist bunt und ansprechend gestaltet und steht vor einem Jobcenter-Gebäude in der Regio04 als »Außensensor« (Interview02Regio04). In dem Bus sind Beschäftigte des Maßnahmenträgers, die alle eintretenden Jugendlichen beraten, unabhängig davon, ob sich diese im SGB-II-Regelkreis befinden. Dabei reicht die Beratung von allgemeinen Fragen bis zum Prüfen von Bewerbungsunterlagen. Der Bus, der als offenes Angebot vor dem SGB-II-Träger (und

78 Diese Notunterkunft wird nicht über den Eingliederungstitel finanziert. Während das Jobcenter 30 Prozent für die Maßnahme aufwendet, stammen 70 Prozent aus Bundesund ESF-Mitteln, aber auch ehrenamtliche Arbeit (Handwerker, Ordensmitglieder) und Sponsorengelder werden in den Projekten genutzt (Interview01Regio04, Interview03Regio04). 
in Rücksprache mit diesem) angesiedelt ist, fungiert jedoch auch als Steuerungselement. Denn der Maßnahmenträger fragt beim Jobcenter an, ob die Jugendlichen, die den Beratungsbus wie auch den offenen Bereich nutzen, dem SGB-IITräger bereits bekannt sind und gegebenenfalls welche SGB-II-Träger-Fachkraft ihn betreut, um das weitere Vorgehen abzustimmen. In dieser Regio etabliert sich im Bereich der Beschäftigungsförderung ein sozialarbeiterisches Netzwerk: »Es funktioniert total unkompliziert und automatisch ist schon ein kleines Netzwerk um den Kunden, ohne dass der das merkt. Was ja sonst immer schwierig ist, dieses Netzwerk um ihn aufzubauen.«(Fallmanagerin, Interview02Regio04)

Gefordert wird, wenn die Personen an der Maßnahme teilnehmen, dass bestehende Hemmnisse zur Kooperation mit der Arbeitsverwaltung abgebaut sowie das Aufsuchen von unterstützenden Leistungen externer Dienstleister (Sucht-, Schuldner-, Psychosoziale Beratung, Wohnraumbeschaffung, Jugendgerichts-, Bewährungshilfe etc.) vorgenommen werden. Zum Teil werden die Teilnehmenden auch durch SozialarbeiterInnen oder SozialpädagogInnen aus den Maßnahmenträgern zu den unterstützenden Stellen gebracht, um sicherzustellen, dass diese Leistungen in Anspruch genommen werden. Hier gibt es teilweise Konfliktpotentiale, wenn sich die Personen etwa bei bestehenden Suchterkrankungen gegen solche Angebote wehren, der Maßnahmenträger jedoch seitens des SGBII-Trägers dazu angehalten wird, diesen Schritt zu gehen (Interview08Regio13). Suchtberatungsstellen haben hier meist den Grundsatz, nur freiwillig erscheinende Personen zu beraten und therapieren, da sonst eine zu hohe Rückfallquote erwartet wird. Die Bemühungen wider die Süchte sind nicht so erfolgreich: »Entgiftung anfangen abbrechen (»Ja ja« (Fallmanagerin, Interview04Regio04)) anfangen abbrechen. (Sozialpädagoge, Interview04Regio04)

Es zeigt sich in allen drei Interventionsoptionen das fördernde Element in der Herausbildung eines sozialarbeiterischen Netzwerkes bis in die unmittelbare Lebenssphäre der SGB-II-Leistungsbeziehenden hinein, um eine Erreichbarkeit herzustellen. Das Fordernde drückt sich in der, dann vehement und in Face-toFace-Interaktion, eingeforderten Erreichbarkeit für die Arbeitsverwaltung und den Maßnahmenträger aus. Daneben sollen die Teilnehmenden offen über Problemlagen sprechen und diese im wechselseitigen Austausch, soweit möglich, beseitigt werden. 


\section{Integrationsform}

Die soziale Teilhabe soll hier insbesondere über eine stärkere Kooperation mit der Arbeitsverwaltung hergestellt werden, zu der vertrauensfördernde Maßnahmenelemente ${ }^{79}$ motivieren sollen:

»da diente ja das gemeinsame Essen, die gemeinsame Mahlzeit auch den sozialen, den sozialen Kontakten, nee. Beim Essen öffnet man sich, man ist kommunikativer, ja, da kann man das eine oder andere Problem besprechen, das man normalerweise unter normalen Umständen nicht besprechen würde« (Maßnahmenträger-Standortleiterin, Interview04Regio07).

Quasi-offene Elemente, die einerseits als Nebengespräche und -schauplätze eine vertrauensfördernde Komponente besitzen, dienen gleichzeitig dem Öffnen der Teilnehmenden und der Personenanalyse. Das Fallmanagement sowie der Maßnahmenträger haben die Aufgabe, die Hintergründe zu ermitteln, um bei der Problemlösung möglichst zielführend zu unterstützen. »Das ganze Sozialverhalten wird da abgeklärt und daran wird ja dann gemessen, ist das jetzt ein sehr schwer beeinträchtigter Jugendlicher oder nur ein >Leichter«.« (Fallmanagerin, Interview02Regio04) Von Interesse ist beispielsweise die Schuldenhöhe, wer sind die Gläubiger, wie ist die Wohnsituation, wie ist das familiäre/soziale Umfeld beschaffen? Diese Informationen werden jedoch eher in einer vertrauensvollen Umgebung von den SGB-II-Leistungsbeziehenden ausgesprochen. »Wenn man so ein sensibles Klientel hat, da kann man nicht mit Forderungen und Holzhackermethodik vorgehen, da muss man schon ein bisschen sensibler mit umgehen.« (Fallmanagerin, Interview02Regio04) Die Arbeitsvermittlerin (Interview02Regio04) führt illustrierend den Rechenschaftsbericht des christlichen Jugendträgers an, der das »Herstellen von Vertrauen und Selbstvertrauen» bezweckt. Dabei vertreten die Maßnahmenträger-Beschäftigten das Ideal, die Jugendlichen voraussetzungslos anzunehmen, um ihnen nahezubringen, sich selbst und ihr Gegenüber ebenso zu akzeptieren.

»Man muss sich immer wieder, als Mitarbeiter hier im Haus, in den Sinn rufen, dass sie, dass sie erwachsen sind und dass man sie nicht behandeln darf wie Kinder. Weil das haben sie in ihrem Leben so oft und immer wieder erlebt und das man sie auch wirklich halt aufbaut. Und ihnen auch sagt: ihr seid erwachsene Menschen und ihr müsst das in die Hand nehmen und euern Leben selbst in die Hand nehmen, aber dazu brauchen sie halt

79 Vergleiche Interview02Regio04, Interview02Regio12. 
noch ganz ganz viel Unterstützung. [...] aber manche muss man halt auch so ein bisschen, ich sag jetzt nicht zwingen, aber so ein bisschen >schubsen`, sie sich zu holen.» (Sozialpädagogin, Interview03Regio04)

\section{Aktivierungsform}

Der Aktivierungsdiskurs wird hier über eine Aktivierung in vertrauensschaffende und zugleich kontrollierende Instrumente ausgeführt. Die Weckung von Vertrauen zum SGB-II- und Maßnahmenträger soll zur Kommunikation führen. Die Kehrseite der, häufig von den Fachkräften in einer sehr wohlwollenden Art, artikulierten Unterstützung und anvisierten Vertrauensbildung ist eine paternalistische Seite, durch die staatliche Kontroll- und Erziehungsfunktion bis tief in die Persönlichkeit betreffende Bereiche hineinwirkt. Diese Einsichtnahme ist weit umfassender als die übliche Kommunikation mit SGB-II-Leistungsbeziehenden, die ohnehin erreichbar für die AkteurInnen der Beschäftigungsförderung sind. Der Versuch, sich der Kommunikation mit dem SGB-II-Träger zu entziehen, führt über die sozial aktivierenden Maßnahmen nachgerade in sein Gegenteil.

Zusammengefasst verläuft die Integration in die Ordnung der Arbeitsverwaltung über alternative und neu geschaffene Kommunikationswege, um die Erreichbarkeit mit einer bisher unerreichbaren Klientel überhaupt erst herzustellen. Integriert wird über sozialarbeiterische und offen-anmutende, attraktive Angebote in augenscheinlich vertrauensvollen und institutionsfreien Zonen der Beschäftigungsförderung. Die Aktivierung zu Vertrauen birgt jedoch auch eine inhärente Kontrolle, die vordergründig nicht unbedingt sichtbar ist.

Tabelle 7: Typ Ordnung der Arbeitsverwaltung

\begin{tabular}{|l|l|}
\hline Kategorien/Typen & Ordnung der Arbeitsverwaltung \\
\hline Maßnahmenziel & $\begin{array}{l}\text { Kontakt und Kommunikation mit spezifischen } \\
\text { Personengruppen (wieder-) herstellen }\end{array}$ \\
\hline $\begin{array}{l}\text { Sicht der Arbeitsverwaltung } \\
\text { auf die Maßnahmenteilneh- } \\
\text { menden }\end{array}$ & $\begin{array}{l}\text { Für Akteure der Arbeitsverwaltung unerreich- } \\
\text { bare Individuen }\end{array}$ \\
\hline Elemente des Förderns & $\begin{array}{l}\text { Sozialarbeiterisches Netzwerk, das in >institu- } \\
\text { tionsfreier Kommunikation`, Vermittlungs- } \\
\text { hemmnisse klärt und bei der Behebung von } \\
\text { Problemlagen unterstützt }\end{array}$ \\
\hline
\end{tabular}




\begin{tabular}{|l|l|}
\hline Elemente des Forderns & $\begin{array}{l}\text { Erreichbarkeit bis hin zu Face-to-Face-Inter- } \\
\text { aktionen im Lebensumfeld der Teilnehmen- } \\
\text { den, Offenheit im Sprechen über Problemla- } \\
\text { gen Initiative diese zu beseitigen }\end{array}$ \\
\hline Integrationsform & $\begin{array}{l}\text { Vorstufe zur Arbeitsfähigkeit; Motivation zur } \\
\text { Erreichbarkeit sowie zur Kooperation }\end{array}$ \\
\hline Aktivierungsform & Vertrauen/Überwachung \\
\hline
\end{tabular}

Quelle: Eigene Darstellung

\subsubsection{Typ Ordnung des gesellschaftlichen Miteinanders}

\section{Maßnahmenziel}

In dem Typ der Integration in eine Ordnung des gesellschaftlichen Miteinanders ist das zugesprochene Ziel, die Regeln der sozialen Interaktion (wieder-) zu erlernen und anzuwenden. Darunter wird insbesondere das Verhalten innerhalb und durch die Gruppe der Maßnahmenteilnehmenden verstanden. Es umfasst gegenseitiges Unterstützen in der Gruppe, einen gruppenfähigen Umgang mit Aggressionen, ${ }^{80}$ allgemeine Umgangsformen wie das Anklopfen vor dem Eintreten $^{81}$ sowie die Pflege des äußeren Erscheinungsbildes: ${ }^{82}$

»Lernen früh aufzustehen, lernen gewaschen zu sein, lernen ordentliche Klamotten anzuhaben, lernen ordentliche Schuhe anzuhaben, lernen den Tag über nicht zu Hause zu sein [...], aber trotzdem mein Leben geregelt zu kriegen.» (Teamleiterin, Interview04Regio02).

In diesem Typ werden grundlegende Kulturtechniken, die auch die Interaktion in sozialen Gruppen und in potentiellen Arbeitszusammenhängen prägen, vermittelt. Köperpflege und Schönheitshandeln (verstanden als Beachtung kultureller Regelstandards) werden durch Module gefördert, in denen basale Kompetenzen

80 Die Aggressionskontrolle spielt eine Rolle in den U25-Maßnahmen in Regio02 (Interview01Regio02), in dem Telefoninterview01Regio03 und im Memo01Regio04.

81 Zum Beispiel Interview03Regio10.

82 Vergleiche Telefoninterview01Regio03, Interview02Regio04, Telefoninterview01Regio04. 
der Haushaltsführung wie Wäschebügeln thematisiert werden, die aber auch Typ-Beratungen und Styling-Tipps umfassen können: ${ }^{83}$

»Ja so Wäschepflege. Also es klingt eigentlich lustig, im Prinzip und es ist eigentlich schade, dass man Menschen, die 50 Jahre alt sind lernen muss, wie man Wäsche wäscht, wie man Wäsche bügelt und sie richtig zusammenlegt, damit man dann wenn man's braucht anziehen kann« (Teamleiterin, Interview04Regio02).

Die Pflege des Äußeren und die Kompetenz, dafür notwendige Ressourcen bereitzuhalten, umfassen jedoch auch Strukturen der Sozialwirtschaft und der Armenfürsorge. Beispielsweise werden Maßnahmenteilnehmenden über den Maßnahmenträger in Regio13 (Interview03) von Friseur-Lehrlingen aus schulischen Bildungsmaßnahmen die Haare geschnitten, der Zugang zu einer Einrichtung für den günstigen Kauf von Kleidung (Sozialkaufhaus) und Lebensmitteln (regionale Tafel) hergestellt. Diese grundsätzlichen Voraussetzungen müssten bestehen, ehe Praktika, Weiterbildung oder eine Erwerbsintegration erfolgen könnten. Die Perspektive auf günstige Lebenshaltungskosten wird in diesem Typ allerdings ausschließlich als Ermöglichung einer Steigerung der Herzeigbarkeit von Personen betrachtet. Könnte jedoch auch als Zeugnis der Armut von SGB-IILeistungsbeziehenden betrachtet werden, wie sie die Debatte um den Ratgeber des Jobcenters Pinneberg zu Tage förderte, in dem nachdrücklich diskutierte Spartipps ausgegeben werden, wie etwa nicht hungrig Lebensmittel einkaufen zu gehen (Öchsner 2013).

Gruppenfähigkeit wird dabei nicht nur als Ziel der Intervention verstanden. Gruppendynamiken werden vielmehr dazu genutzt, um andere Ziele zu erreichen (was wiederum die Gruppenfähigkeit voraussetzt).

»Ja, auch dieser Druck, den einfach die Gruppe dann auf jemanden auch ausüben kann, der soll da auch ein wenig genutzt werden. So wie, jetzt kommst du heute schon wieder ungewaschen oder jetzt kommst du heute schon wieder mit dem gleichen T-Shirt. Gestern haben wir dir doch gesagt, du sollst ein anderes anziehen. Also das was praktisch die Gruppe nicht immer jemand, der von oben runter, der über denen steht, weil der Lehrgangsleiter steht halt nun mal vom Niveau und von allem über den den Leuten, aber so diesen Gruppendruck den nutzt man dann. Deswegen hat man dann auch eine relativ klei-

83 Durchgängige Module im Sample, insbesondere im Aktivcenter (exemplarisch Interview01Regio01, Interview04Regio02, Interview01Regio11). 
ne Gruppe gekauft, nur mal mit 16 Leuten und das reicht aufwärts.« (Teamleiterin, Interview04Regio02)

Gruppen-internes Lob und Tadel seien dabei »ohne Wertung. Das kommt auch besser an.« (Maßnahmenträger-Standortleiterin, Interview04Regio07).

\section{Sicht der Akteurinnen und Akteure}

Gemäß der Sicht der AkteurInnen der Arbeitsverwaltung auf SGB-II-Leistungsbeziehende dieses Funktionstypen sind die Maßnahmenteilnehmenden Personen, die überwiegend wenig sozialen Kontakt haben und Vereinsamungstendenzen aufweisen. Beschrieben werden hier fehlende Umgangsformen sowie ein mangelhafter Umgang mit Aggression. Auch das Äußere sei auffällig, da sich diese Individuen körperlich und hygienisch nicht pflegen.

»Einer, dem die zwei Schneidezähne fehlen, der kann sich zwar vorstellen, den kannst du auch Bewerbungstraining machen lassen und IT-Systeme erlernen lassen, das bringt nichts. [...] es gibt nicht nur Leute, die sich nicht waschen, sondern es gibt Leute, die einfach vereinsamt sind und die es schwer haben und wo man sieht, übergewichtig, also adipös, oder irgendwie schon von außen hin schon Hemmnisse aufzeigen, wo der Fallmanager sagt, wer kommt denn da, da werde ich ja die nächsten 20 Jahre gar nicht mehr quitt.« (Zentraler Projektmanager, Interview03Regio07)

Falls die Teilnehmenden auf soziale Netzwerke zurückgreifen könnten, seien es eher Umfelder, die von Erwerbslosigkeit dominiert werden.

\section{Elemente des Förderns und Forderns}

Das fördernde Element ist in diesem Typ die Ermöglichung von moderiertem Kontakt mit anderen Personen sowie Gelegenheiten für Gruppenlernen zu bieten. Infolgedessen sollen soziale Kompetenzen sowie soziale Netzwerke für eine eventuelle spätere gegenseitige Unterstützung aufgebaut werden. Außerdem wird Wissen zu allgemeinen Umgangsformen und der Körperlichkeit (Körper-, Kleidungspflege, Typberatung) weitergegeben. Das Fordernde drückt sich hier in der Offenheit für das Arbeiten an Bedingungen der eigentlich persönlichen Sphäre aus, um einen Erwerbseinstieg gewährleisten zu können. So wird das individuelle Erscheinungsbild zum Thema der Arbeitsverwaltung:

»Also Herr oder Frau XY, tut mir leid, wenn ich Ihnen das so sagen muss, aber ich finde heute ihr äußeres Erscheinungsbild nicht gerade passend für unser Gespräch heute. Was ist denn los heute? Also schon auch ein bisschen in die Richtung, das man sagt, kann ich 
ihnen irgendwie helfen? Ist irgendwas passiert? Warum haben sie heute das dreckige Ding an? Oder dass man sagt, entschuldigen sie bitte, dass ich sie anspreche, aber sie riechen heute nach Alkohol. Ich muss ihnen das so sagen, ich rieche das heute, was ist denn los?« (Teamleiterin, Interview04Regio02)

Um solch sensible Themen ansprechen zu können, werden Fachkräfte der SGBII-Träger in der Regel in Gesprächsführungstechniken geschult. In Regio02 werden Maßnahmen Sozialer Aktivierung erst vorgeschlagen, wenn mehrfach im SGB-II-Träger Gespräche etwa zum Äußeren geführt wurden. Die Maßnahmenteilnehmenden sind häufig »nicht beglückt, aber sie machen das dann eigentlich schon.« (Teamleiterin, Interview04Regio02)

"Aber wenn sich jemand totweigert und sagt, ich kann das nicht, da werde ich, da kriege ich Depressionen, da werde ich psychisch krank, das kann ich nicht, zwingen sie mich bitte nicht, dann machen wir es eigentlich auch nicht. Aber wenn man versucht jemanden sanft zu überzeugen, dann unterschreibt er aber bitte auch die EV [Eingliederungsvereinbarung; $\mathrm{CF}]$ und dann hält er aber wenn's geht durch und das ist dann ja auch ein persönlicher Erfolg, wenn er sagt, ja ich hab es geschafft, ich habe durchgehalten.« (Teamleiterin, Interview04Regio02)

\section{Integrationsform}

Die soziale Teilhabe wird auch hier in einer Vorstufe zur Arbeitsfähigkeit gefördert, in Maßnahmen die auf Gruppeninteraktionen zielen. Dafür wird in Elemente integriert, die deviantes Auftreten in der sozialen Interaktion wie auch im Erscheinungsbild thematisieren. Über diese Kritik, gekoppelt an konstruktive Verbesserungsvorschläge, soll die Devianz gemindert werden.

\section{Aktivierungsform}

Der Aktivierungsdiskurs wird hier über die Aktivierung zur Sozialkompetenz ausgeführt, »bis hin eben auch: [...] sich als Teil einer Gesellschaft zu fühlen. Nicht immer nur [...] sich selbst zu sehen, sondern auch die Zusammenhänge zu sehen.« (Mitarbeiter Projektmanagement, Interview02Regio13)

Zusammengefasst wird im Typ des gesellschaftlichen Miteinanders in Gruppenstrukturen integriert, um Grundregeln der sozialen Interaktion (wieder-) zu erlernen und sich im sozialen Verhalten sowie der äußeren Erscheinung den normativen Vorstellungen anzupassen. Aktiviert wird zur Sozialkompetenz. 
Tabelle 8: Typ Ordnung des gesellschaftlichen Miteinanders

\begin{tabular}{|l|l|}
\hline Kategorien/Typen & Ordnung des gesellschaftlichen Miteinanders \\
\hline Maßnahmenziel & $\begin{array}{l}\text { Grundregeln der sozialen Interaktion (wieder-) } \\
\text { erlernen (etwa Umgangsformen, Gruppenlernen } \\
\text { und - unterstützung, Pflege des Äußeren) }\end{array}$ \\
\hline $\begin{array}{l}\text { Sicht der Arbeitsverwal- } \\
\text { tung auf die Maßnahmen- } \\
\text { teilnehmenden }\end{array}$ & $\begin{array}{l}\text { Individuen, mit wenig Personenkontakt (tlw. } \\
\text { Vereinsamungstendenzen), die kaum Umgangs- } \\
\text { formen beherrschen und/oder sich körperlich } \\
\text { und hygienisch nicht pflegen }\end{array}$ \\
\hline Elemente des Förderns & $\begin{array}{l}\text { Begleiteter Personenkontakt, Herstellen von } \\
\text { Gruppenlernen, Wissensvermittlung zu allg. } \\
\text { Umgangsformen und Körperhygiene }\end{array}$ \\
\hline Elemente des Forderns & $\begin{array}{l}\text { Offenheit für das Arbeiten an Bedingungen der } \\
\text { eigentlich persönlichen Sphäre, um eine Interak- } \\
\text { tion in Gruppen gewährleisten zu können }\end{array}$ \\
\hline Integrationsform & $\begin{array}{l}\text { Vorstufe zur Arbeitsfähigkeit; sozialkompetentes } \\
\text { Handeln und Minderung devianten Auftretens }\end{array}$ \\
\hline Sozialkompetenz
\end{tabular}

Quelle: Eigene Darstellung

\subsubsection{Typ Ordnung des aktiven Subjekts}

\section{Maßnahmenziel}

Im Typ der Integration in die Ordnung des aktiven Subjekts sind die normativen Strukturen der aktiven Persönlichkeit von Bedeutung. Es geht um Persönlichkeits- und Selbstwirksamkeitsförderung und das Entdecken individueller Stärken. Über anspruchsarme und häufig kreative Angebote wie Theatergruppen, Laienzirkus, Filmprojekte, Rap-Programm, Tanzgruppen, Sportangebote, Ferienfreizeiten für Jugendliche, Kurse zum künstlerischen Gestalten, Nähkurse mit ei- 
ner künstlerischen Ausrichtung ${ }^{84}$ sollen nicht unmittelbar arbeitsmarktnahe Fähigkeiten entdeckt und die Selbstwirksamkeit gestärkt werden. Die so erworbene Selbstwertsteigerung soll zur weiteren Maßnahmenteilnahme motivieren, zur arbeitsmarktlichen Fokussierung beitragen und erste Schritte in Richtung Erwerbstätigkeit erbringen. Insofern sollen alle Rahmenbedingungen geschaffen werden, damit die Personen ein Ziel fokussieren und die Kenntnis über die dafür notwendigen Schritte vermittelt werden.

»Wir versuchen die Teilnehmer immer in die Aktivität mit einzubeziehen, a) ist das eine Motivation für sie, das was sie auch produzieren oder herstellen, dass es anerkannt wird oder und dann sag ich immer, wenn sie was Schönes gemacht haben, werden die auch gelobt. Lob und Tadel, ja und dann kommt man in Berührung mit den Teilnehmern, dann fragt man, was gefällt ihnen auch so, also quasi die Arbeit an der Basis« (Maßnahmenträger-Standortleiterin, Interview04Regio07).

Daneben sollen eigene Grenzen ausgeweitet beziehungsweise überwunden werden, um die Persönlichkeitsentwicklung zu ermöglichen beziehungsweise zu stimulieren. Dies ist etwa die zentrale Ausrichtung einer Maßnahme für ehemalige Drogenabhängige, deren Hauptinhalt in einer Gebirgsüberquerung besteht (Interview04Regio02).

\section{Sicht der Akteurinnen und Akteure}

Die Sicht der AkteurInnen der Beschäftigungsförderung auf SGB-II-Leistungsbeziehende beschreibt hier Personen mit wenig Selbstvertrauen, die auf die eigenen Fähigkeiten fokussiert werden müssten. Diese Kompetenzen können berufsrelevant sein, bei arbeitsmarktfernerer Klientel sollen dagegen andere Fertigkeiten das Selbstbewusstsein soweit aufbauen, dass die insgesamt gewachsene und gefestigte Persönlichkeit dadurch zur Erwerbstätigkeit motiviert werde. So zentriert sich auch der Umgang mit der persönlichen Berufsbiografie im Profiling auf die Stärken der Person: »Das Allerwichtigste ist, dass man den Menschen zuhört, auch das ist eine neue Erfahrung für Viele, nicht, das als Mensch

84 Gefördert werden in diesem Typus also künstlerische, kulturelle und sportliche Maßnahmenanteile, wie Theater- und Tanzprojekte (Interview04Regio02, Interview04Regio04, Interview02Regio13, Interview04Regio12), ein Kommunikationskurs mit der Ausrichtung auf Selbst- und Fremdwahrnehmung (Interview04Regio07), Airbrush, Aquarellmalerei (Interview04Regio07), Musikproduktionen (Interview03Regio04, Interview04Regio04) und Basteln (Interview02Regio11). 
angenommen wird, wertgeschätzt wird, dass das nicht alles nur Mist war, was er erlebt hat und gemacht hat, diese negativen Suggestionen, dass schaffst Du sowieso nicht« (Maßnahmenträger-Standortleiterin, Interview04Regio07).

Auch Gruppenprozessen wird die Steigerung der individuellen Motivation zur Erwerbsintegration zugesprochen. Erst durch diese würden sich vielfach die Maßnahmenteilnehmenden für Neues öffnen: »Der Mensch muss erst bereit sein, sich auf etwas Neues einzulassen und das auch wollen. Und das alles ihm bewusst zu machen, [...] Du kannst viel, schau hin, was Du alles kannst.« (Maßnahmenträgerleiterin, Interview01Regio02)

Durch die Teilnahme an den sozial aktivierenden Bausteinen erhöhe sich, über eine gewisse Zeit hinweg, die Motivation zur generellen Maßnahmenteilnahme und Bausteinen wie Bewerbungstraining und Coaching (zum Beispiel Interview04Regio12). Die niederschwelligen Module haben dabei nicht nur die Funktion, die soziale Interaktion in der Gruppe zu entwickeln und die Motivation zur gesamten Maßnahmenteilnahme zu fördern, sondern insbesondere über arbeitsmarktferne Tätigkeiten das Selbstbewusstsein im Allgemeinen zu stärken. Auf Basis dieser Stabilisierung könne dann erst das Thema Erwerbsarbeit bearbeiten werden.

»Wir haben ein Improvisationstheater, dient auch dazu Selbstbewusstsein zu stärken, Mut zu machen, Motivation zu finden, wo vielleicht bisher keine war, ja einfach, dieses Selbstbewusstsein, jetzt geh ich und bewerbe mich mal da und ich bin was wert und das zu stärken.« (Teamleiterin, Interview04Regio02)

Über die Realisierung von eigenen Kompetenzen in eher arbeitsmarktfernen Bereichen wie im Improvisationstheater oder einem Tanzkurs (Interview04Regio12) sollen SGB-II-Leistungsbeziehende so weit über die Steigerung des Selbstbewusstseins stabilisiert werden, dass sich auch eine größere Eigeninitiative zur Erwerbsaufnahme entwickelt. »Dass die Teilnehmer entdecken, welche Potentiale sie haben, weil sie hier einfach die Möglichkeit bekommen. Schauen sie mal, sie können hier also malen, also sich da da beteiligen an irgendwelchen Projekten« (Maßnahmenträger-Standortleiterin, Interview04Regio07).

Die Persönlichkeitsförderung soll in ihrem fordernden Element der Erwerbsaufnahme dienen und während beziehungsweise nach dieser Maßnahme müssen konkrete Schritte zur Erwerbsaufnahme unternommen werden, so die Maßgabe von SGB-II- und Maßnahmenträger. 


\section{Integrationsform}

Soziale Teilhabe soll hier über die Selbstwirksamkeit erhöhende Tätigkeiten begünstigt werden. Die Ausrichtung auf die individuellen Stärken (auch wenn zum Teil in vordergründig arbeitsmarktirrelevanten Bereichen) und das schrittweise Erhöhen des eigenen Limits sollen einen Sinn und Freude im Leben geben. Darauf aufbauend könnten die Arbeitskompetenzen gesteigert werden.

»Und dann wenn jemand so bisschen mutiger ist, hat er auch oft einmal den Mut zu sagen, okay, ich bewerbe mich einfach noch mal, auch wenn das jetzt vielleicht noch mal 50 mal schief geht, aber vielleicht habe ich ja auch irgendwo mal Glück oder ich gehe weiter jetzt, ich mach jetzt mal ein Praktikum in einer Firma.« (Teamleiterin, Interview04Regio02)

Auf die Nachfrage, ob diese Maßnahme dann immer in eine Praktikums- oder Stellensuche eingebunden ist, erklärt die Gesprächspartnerin:

»Aber das ist nicht das vorrangige Ziel. Wenn man grad auch Menschen in der Situation überfordert, dann machen die ganz schnell zu und dann sitzen die wieder zu Hause in ihrem Schneckenhaus und gehen nicht mehr raus. Also das ist schon langsam, schon eins nach dem anderen und eben auch mit einer kleinen Gruppe« (Teamleiterin, Interview04Regio02).

Insgesamt ist auch bei den auf Selbstwirksamkeit ausgelegten Tätigkeiten ein langfristiger Bezug zum Arbeitsmarkt vorhanden, indem diese immer als Vorstufe zu weiteren Maßnahmen oder begleitend zu Qualifizierungselementen durchgeführt werden:

»Wenn die Maßnahme gar nie im Mindesten auf Arbeit rausläuft, dann wird's auch glaub ich nicht so breite Zustimmung unter den pAps [SGB-II-Träger-Fachkräfte - die sogenannten persönliche Ansprechpartner; CF] geben. Es muss schon also ein Ziel dahinter sein. Also jetzt nur das ich sag, also Kunde kommt, zack, der wurde gemobbt, danach drei Monate zeichnen, würde bei unserer Front jetzt nicht auf breite Zustimmung stoßen, weil man immer sehen will, was kommt denn nun danach. Wenn er dann drei Monate gezeichnet hat, was kann er denn dann danach? Ist er denn dann soweit, dass er sich wieder zum Teil wenigstens selber finanzieren kann?« (Fallmanagerin, Interview02Regio06)

\section{Aktivierungsform}

Der Aktivierungsdiskurs wird in diesem Typ über die Aktivierung der Maßnahmenteilnehmenden zur Selbstwirksamkeit ausgeführt. 
Zusammenfassend zielt die Integration in die Ordnung des aktiven Subjekts auf Personen mit Defiziten im Selbstvertrauen, um über überwiegend arbeitsmarktferne kreative Tätigkeiten die Selbstwirksamkeit zu fördern und damit so weit zu stabilisieren, dass es einer späteren Erwerbsaufnahme förderlich ist.

Tabelle 9: Typ Ordnung des aktiven Subjekts

\begin{tabular}{|l|l|}
\hline Kategorien/Typen & Ordnung des aktiven Subjekts \\
\hline Maßnahmenziel & $\begin{array}{l}\text { Persönlichkeitsförderung; Selbstwirksamkeit } \\
\text { (wieder-)herstellen, positives Feedback indi- } \\
\text { viduell sowie in Gruppen }\end{array}$ \\
\hline $\begin{array}{l}\text { Sicht der Arbeitsverwaltung } \\
\text { auf die Maßnahmenteilneh- } \\
\text { menden: }\end{array}$ & $\begin{array}{l}\text { Individuen mit wenig Selbstvertrauen, die ei- } \\
\text { gene Fähigkeiten nicht wahrnehmen (können) }\end{array}$ \\
\hline Elemente des Förderns & $\begin{array}{l}\text { Persönlichkeitsförderung über die Fokussie- } \\
\text { rung auf individuelle Fähigkeiten soll zur Er- } \\
\text { werbstätigkeit motivieren; >Wohlfühlangebo- } \\
\text { te<: Künstlerisch, Kulturell, Sport, Strategien } \\
\text { zur Problembehebung aufzeigen }\end{array}$ \\
\hline Elemente des Forderns & $\begin{array}{l}\text { Einsicht und Motivation zur (späteren) Er- } \\
\text { werbsaufnahme sollte erkennbar sein }\end{array}$ \\
\hline Integrationsform & $\begin{array}{l}\text { Vorstufe zur Arbeitsfähigkeit; Entdecken von } \\
\text { Stärken (ggf. vordergründig nicht arbeits- } \\
\text { marktrelevant); einen Sinn und Freude im } \\
\text { Leben geben, um dann weiter Arbeitskompe- } \\
\text { tenzen fördern zu können }\end{array}$ \\
\hline Selbstwirksamkeit \\
\hline Aktivierungsform
\end{tabular}

Quelle: Eigene Darstellung 


\subsubsection{Vergleich der Funktionstypen im Hinblick auf die Rahmung des Aktivierungsdiskurses}

Der Aktivierungsdiskurs geht von aktiven Subjekten aus, den Kundinnen und Kunden. Weiter entspricht es der Sicht des Diskurses, dass sich das Individuum über Erwerbstätigkeit selbst verwirklicht und so am sozialen Leben teilnimmt. Dagegen erscheinen erwerbsfähige Sozialleistungsbeziehende als deviant. Denn diese soziale Gruppe unterliegt einem inhärenten Vorwurf: der (intentionalen) Nutzung sozialstaatlicher Strukturen, um ein inaktives Leben zu führen. Autonomie wird hier als Synonym für Eigenverantwortung verwandt und die Kategorie der Beschäftigungsfähigkeit vornehmlich auf individuelle Komponenten reduziert, während arbeitsmarktliche Strukturen einen geringeren Stellenwert einnehmen. Die AkteurInnen der Beschäftigungsförderung müssten demnach das aktive Subjekt eher beraten als für dieses tätig werden. Doch in den Maßnahmen Sozialer Aktivierung werden die Maßnahmenteilnehmenden in vielfältiger Weise direkt unterstützt. So diffundiert die im Aktivierungsdiskurs vorherrschende Funktion der Erwerbsintegration in der Praxis in normative Ordnungen der Integration, wie sie zuvor dargestellt wurden: in den Ordnungen 1.) der Tätigkeit, 2.) einer erwerbstätigen Familie, 3.) der Arbeitsverwaltung, 4.) des gesellschaftlichen Miteinanders und 5.) des aktiven Subjekts.

Somit wird der Aktivierungsdiskurs auf unterschiedliche Weisen ausgeführt und damit in seinen Wissensstrukturen, wie auch den normativen Strukturen ausgeweitet. Dies wird zum einen deutlich an den unterschiedlichen Maßnahmenzielen, die verfolgt werden. Während der Aktivierungsdiskurs vordergründig auf eine Integration in Erwerbstätigkeit ausgerichtet ist, wird diese in den hier vorliegenden Maßnahmen Sozialer Aktivierung kaum erreicht. Vielmehr werden weitere Ziele verfolgt, um eine grundsätzlich sehr arbeitsfern beschriebene Klientel zu aktivieren. Die Klientel gilt als schulisch und beruflich niedrig qualifiziert und mit zahlreichen psychischen, physischen, familialen, finanziellen oder juristischen Problemlagen belastet. Die SGB-II-Leistungsbeziehenden, denen Maßnahmen Sozialer Aktivierung zugedacht werden, scheinen dabei weit vom Bild der eigenständig und selbstverantwortlich agierenden Kundin und Kunden entfernt zu sein. Schließlich werden diese in ihrer Maßnahmenteilnahme auf unterschiedlichsten Ebenen unterstützt. Die Schilderungen der Maßnahmenklientel sind hier über alle Typen hinweg annähernd homogen und decken sich mit den Schilderungen zu Hemmnissen in Teilen der SGB-II-Klientel aus anderen Studien (Kaltenborn/Kaps 2013, Schütz et al. 2011, Henkel et al. 2009). ${ }^{85}$ Die In-

85 Vergleiche Kapitel 5.4.5 Verfestigte Arbeitslosigkeit: Eine arbeitsmarktferne Klientel. 
tegration in Erwerbstätigkeit scheint hier an Grenzen der individuellen Beschäftigungsfähigkeit und arbeitsmarktlichen Nachfrage zu stoßen. Dies zeigt sich etwa daran, dass schon zum Zeitpunkt der Maßnahmenkonzeption durch AkteurInnen der Arbeitsverwaltung, die Maßnahmen statt der Erwerbsintegration auf den Aufbau von 1.) Arbeitstugenden und -strukturen, 2.) Rahmenbedingungen für Erwerb in der Familie, 3.) Kontakt zur Arbeitsverwaltung, 4.) Grundregeln sozialer Interaktion und 5.) Persönlichkeitsförderung zielen (siehe Abbildung 9: Maßnahmenziele).

Verbunden mit den unterschiedlichen Ausrichtungen der Maßnahmenziele liegt in den jeweiligen Integrationsformen ein wesentlicher Unterschied zwischen den Typen. Die Funktionstypen der Integration in die Ordnung der Tätigkeit und die Ordnung der erwerbstätigen Familie stehen der Erwerbsintegrationsforderung des Aktivierungsdiskurses am nächsten. Ihre Nähe zum Diskurs wird in der Erstnennung dieser Typen ausgedrückt.

\section{Abbildung 9: Maßnahmenziele}

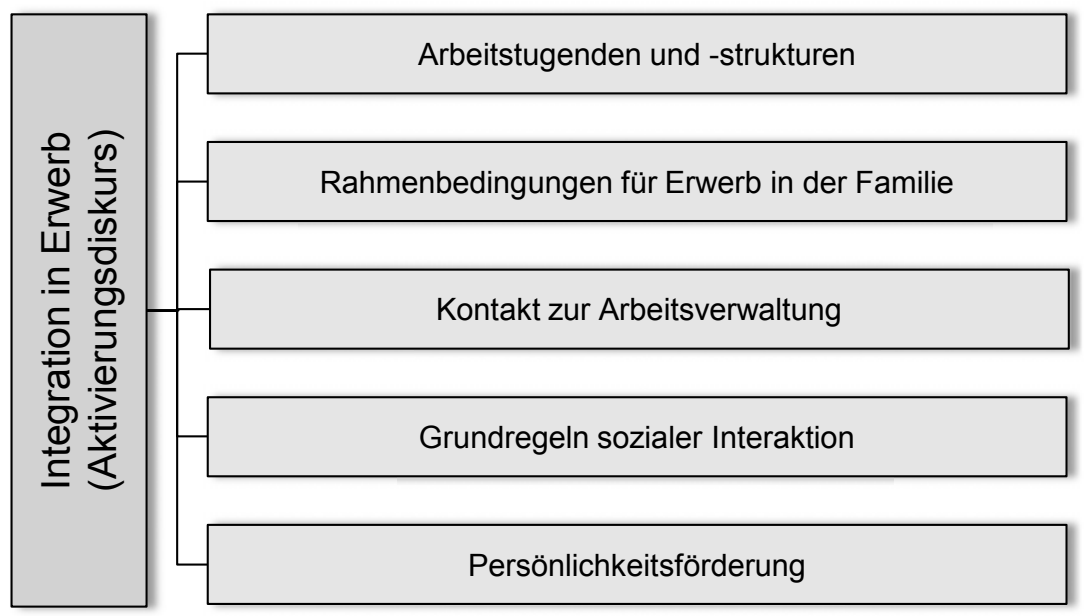

Quelle: Eigene Darstellung

Letztlich gelingt auch in der Integration der Ordnung der Tätigkeit und der erwerbstätigen Familie überwiegend keine Erwerbsintegration, sondern eine Aktivierung zur Tätigkeit für die Subsistenz beziehungsweise die Simulation von Erwerbstätigkeit. Damit bleiben letztlich eine anschließende Maßnahme respektive die Suche nach einer Erwerbsintegration weiterhin notwendig. Die Typen der Integration in die Ordnung der Arbeitsverwaltung, des gesellschaftlichen Miteinanders und des aktiven Subjekts haben eine beträchtliche Orientierung hin 
zu Intentionen der Sozialarbeit. Sie sind stärker unterstützend sowie durch eine intensivere Interaktion fokussierter auf die individuelle Problemlösung, indem sie persönlichkeitsstabilisierend ausgerichtet sind und Vermittlungshemmnisse bearbeiten sollen, die von den AkteurInnen der Beschäftigungsförderung diagnostiziert werden. Somit vollzieht sich die Aktivierung in einer Art Learning-byDoing in den Bereichen, die (wieder-)erlernt werden, um anschließend deren Einhaltung zu überprüfen.

Im Typ der Ordnung der Tätigkeit soll sich die Integration über die Tätigkeit vollziehen und dockt damit am Erwerbsprinzip des Aktivierungsdiskurses und seiner Ausrichtung auf die soziale Teilhabe über Erwerb an. ${ }^{86}$ Die Aktivierung zur Tätigkeit verspricht so, sich von Erwerbsdistanz und Bewerbungsfrustration über die Betätigung selbst zu entledigen und Lern- sowie Arbeitsmotivation daraus zu generieren.

Im Typ der Ordnung der erwerbstätigen Familie wird die Integration in Tätigkeiten gefördert, die berufsähnliche Rahmenstrukturen beinhalten und selektive Bezüge zur Erwerbsarbeit herzustellen vermögen. So wird zu einer Simulation von Erwerbstätigkeit aktiviert und damit auf Vorstellungen des Aktivierungsdiskurses zur Familie rekurriert: ${ }^{87}$ Die Familie soll füreinander einstehen. Dies meint im Feld, Erwerbstätigkeit und damit die Selbstverwirklichung mindestens einer Person zu ermöglichen, etwa indem Großeltern, Ehepartner oder Geschwister bei der Kinderbetreuung unterstützen und die Rahmenbedingungen wie die Erledigung des Haushalts, der Lebensmittelbeschaffung etc. mit erledigen. Allerdings werden in der Praxis der Arbeitsverwaltung die eigentlich vom familiären Netzwerk erwarteten Rahmenstrukturen, die Erwerbstätigkeit ermöglichen, indem SozialarbeiterInnen hinzugezogen werden und die Einschaltung weiterer öffentlich-professioneller Institutionen, wie dem Jugendamt in der Maßnahmentätigkeit erlernt selbstständig aufzubauen und aufrecht zu erhalten.

Im Typ der Ordnung der Arbeitsverwaltung soll die Kommunikation mit der Arbeitsverwaltung über ein sozialarbeiterisches Netzwerk und somit eine Aktivierung zu Vertrauen und Kontrolle ermöglicht werden. Über den begleiteten Austausch soll das Vertrauen der SGB-II-Leistungsempfangenden zu den Institutionen der Beschäftigungsförderung ausgebaut werden. Damit geraten das Verhalten sowie die Bemühung zur Erwerbsaufnahme kontrollierbarer.

86 Vergleiche Kapitel 4.4 »Alles ist sozial, was Arbeit schafft«: Soziale Teilhabe über Erwerb.

87 Vergleiche Kapitel 4.5.1 Bedarfsgemeinschaft und Familie: Menschen sollen füreinander einstehen. 
Im Typ der Ordnung des gesellschaftlichen Miteinanders sollen die Vermittlung von sozial kompetentem Handeln und die Verminderung von deviantem Auftreten (im Sinne des Aktivierungsdiskurses) über Gruppenprozesse erlernt und überprüft werden. Gruppeninternes Lob und Tadel und damit die Disziplinierung durch die peer-group sind ein Schlüsselelement in dieser Aktivierungsform, denn dies sei keine Meinung »von oben herab, also ohne Wertung. Das kommt auch besser an." (Maßnahmenträger-Standortleiterin, Interview04Regio07). Die Disziplinierung verlagert sich so von der Arbeitsverwaltung weg. Insgesamt rekurriert der Typ auf die Vorstellungen des Aktivierungsdiskurses, dass Einzelne sozialen Regeln nachkommen sollen, um letztlich für sich selbst zu sorgen. ${ }^{88}$

Im Typ der Ordnung des aktiven Subjekts werden überwiegend arbeitsmarktferne Fähigkeiten des Individuums adressiert, um die Motivation zur Erwerbsaufnahme zu wecken und/oder zu prüfen. Dieser Typ rekurriert auf den Aktivierungsdiskurs, indem das Bild des aktiven Subjekts als Unternehmer seiner selbst ernst genommen wird. Das Selbstvertrauen ist dann für das aktive Subjekt als selbstständiges und selbsttätiges Individuum notwendige Voraussetzung für die Aktivität in der Erwerbsarbeit. Zunächst soll diese Motivation bei der sehr arbeitsmarktfernen Klientel über arbeitsmarktferne Tätigkeiten entwickelt werden, indessen die Selbstwirksamkeit gestärkt und in einem weiteren Schritt das Metathema der Erwerbstätigkeitsaufnahme behandelt werden.

88 Vergleiche hier wie auch im Folgenden hinsichtlich des Subjekts als Unternehmer seiner selbst Kapitel 4.2 »Menschen in Arbeit bringen«: Sicht auf die Leistungsbeziehenden. 
Abbildung 10: Umsetzung der Aktivierung

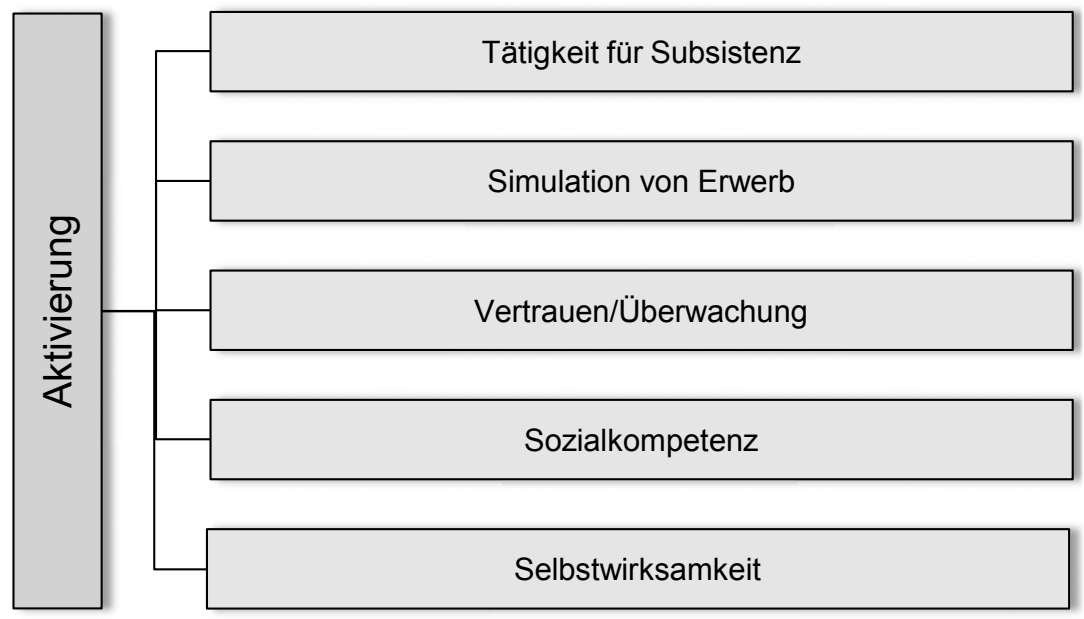

Quelle: Eigene Darstellung

Zusammenfassend zeigt sich, dass die Integration in die Ordnung der Tätigkeit sowie der erwerbstätigen Familie in seiner Funktionsausrichtung dem Aktivierungsdiskurs näher liegt, selbst wenn hier keine Erwerbsintegration erreicht werden kann. In toto nehmen unterstützende Funktionen eine dominante Rolle ein, die immer auch durch eine Prüfung der Mitwirkung und Selbstaktivität begleitet werden. In der Tabelle 10 werden die Typen gesamthaft skizziert. 
Tabelle 10: Vergleich der Funktionstypen

\begin{tabular}{|l|l|l|l|}
\hline Kategorie & $\begin{array}{c}\text { Maßnah- } \\
\text { menziel }\end{array}$ & \multicolumn{1}{|c|}{ Integrationsform } & \multicolumn{1}{|c|}{$\begin{array}{c}\text { Aktivie- } \\
\text { rungsform }\end{array}$} \\
\hline $\begin{array}{l}\text { Ordnung der } \\
\text { Tätigkeit }\end{array}$ & $\begin{array}{l}\text { Tugenden der } \\
\text { Arbeit }\end{array}$ & $\begin{array}{l}\text { Tätigkeiten des täglichen Be- } \\
\text { darfs, mittelbar für den Ar- } \\
\text { beitsmarkt }\end{array}$ & $\begin{array}{l}\text { Tätigkeit für } \\
\text { Subsistenz }\end{array}$ \\
\hline $\begin{array}{l}\text { Ordnung der } \\
\text { erwerbstäti- } \\
\text { gen Familie }\end{array}$ & $\begin{array}{l}\text { Bedingungen } \\
\text { für Berufstä- } \\
\text { tigkeit in der } \\
\text { Familie }\end{array}$ & $\begin{array}{l}\text { Tätigkeit eines Familienan- } \\
\text { gehörigen }\end{array}$ & $\begin{array}{l}\text { Simulation } \\
\text { von Erwerbs- } \\
\text { tätigkeit }\end{array}$ \\
\hline $\begin{array}{l}\text { Ordnung der } \\
\text { Arbeitsver- } \\
\text { waltung }\end{array}$ & $\begin{array}{l}\text { Kontakt und } \\
\text { Kommunika- } \\
\text { tion }\end{array}$ & $\begin{array}{l}\text { Vorstufe zur Arbeitsfähig- } \\
\text { keit; Motivation zur Erreich- } \\
\text { barkeit sowie zur Kooperati- } \\
\text { on }\end{array}$ & $\begin{array}{l}\text { Vertrauen } \\
\text { respektive } \\
\text { Überwachung }\end{array}$ \\
\hline $\begin{array}{l}\text { Ordnung des } \\
\text { gesellschaft- } \\
\text { lichen Mitei- } \\
\text { nanders }\end{array}$ & $\begin{array}{l}\text { Grundregeln } \\
\text { sozialer Inter- } \\
\text { aktion }\end{array}$ & $\begin{array}{l}\text { Vorstufe zur Arbeitsfähig- } \\
\text { keit; sozialkompetentes Han- } \\
\text { deln und Verminderung de- } \\
\text { vianten Auftretens }\end{array}$ & $\begin{array}{l}\text { Sozialkompe- } \\
\text { tenz }\end{array}$ \\
\hline $\begin{array}{l}\text { Ordnung des } \\
\text { aktiven Sub- } \\
\text { jekts }\end{array}$ & $\begin{array}{l}\text { Selbstwirk- } \\
\text { samkeit }\end{array}$ & $\begin{array}{l}\text { Vorstufe zur Arbeitsfähig- } \\
\text { keit; (arbeitsmarktunabhängi- } \\
\text { ge) Stärken entdecken für } \\
\text { späteres fördern der Arbeits- } \\
\text { kompetenzen }\end{array}$ & $\begin{array}{l}\text { Selbstwirk- } \\
\text { samkeit }\end{array}$ \\
\hline
\end{tabular}

Quelle: Eigene Darstellung

\subsubsection{Sozialstaatliche Funktionen}

Wie im Folgenden dargestellt, nehmen Maßnahmen Sozialer Aktivierung auch sozialstaatliche Funktionen ein: Denn sie fokussieren die Förderung der Person in ihrer Ganzheitlichkeit, um eine Beschäftigungsfähigkeit zu erzielen. Letztlich wird so soziale Teilhabe in der Maßnahme ermöglicht und das Individualisierungsprinzip aufgeweicht. Denn diese Maßnahmen verlagern die Intention von 
einer Übernahme der Eigenverantwortung hin zu einer teilweise umfangreichen Unterstützung, obzwar letztlich eine Selbstbefähigung der SGB-II-Leistungsbeziehenden zur Beschäftigung erreicht werden soll. En passant wird so ein dekommodifizierendes Moment installiert, das den Warencharakter der Erwerbstätigkeit mindert, indem zunächst die Problemlagen geklärt werden können, ehe eine Erwerbstätigkeit aufgenommen werden kann und soll. Darüber hinaus ermöglichen die Maßnahmen Sozialer Aktivierung soziale Teilhabe (im >Vorraum der Erwerbsarbeit $`$ ).

Maßnahmen Sozialer Aktivierung sind gerichtet auf eine umfassende Betreuung ihrer Maßnahmenteilnehmenden in Bereichen, die über die originäre Beschäftigungsförderung hinausweisen. In dem Bestreben, alle Sozialleistungen unter einem Dach zu versammeln, respektive aus einer Hand zu vergeben, richten sie sich ganzheitlich auf die Persönlichkeit, anstatt zum Beispiel einzig auf die Qualifikation abzuheben. Mit dem Primärziel zunächst die Beschäftigungsfähigkeit zu fördern, fungieren diese Maßnahmen zudem als Vehikel, das auf unterschiedlichsten Funktionsebenen sowohl zur Maßnahmenteilnahme als auch in langfristiger Perspektive zum Erwerbseinstieg integrieren soll.

Dementsprechend haben die Maßnahmen Sozialer Aktivierung eine ausgeweitete Zielsetzung - sie sind ganzheitlich und werden so vielfach explizit in den Interviews benannt. Tatsächlich wird das Individuum in einer weit umfassenderen Form einbezogen, als allein im Sinne der Erwerbsintegration:

»Chancen sind, dass man den Menschen als ganzen Menschen sieht auch sieht mit seinem ganzen Umfeld sieht, wenn man also nicht nur sieht der Arbeitsmarkt ruft, wir müssen die Menschen fit machen, sondern das man erkennt oder herausarbeitet, wo die Hemmnisse liegen und das man die nach Möglichkeit gleichzeitig angeht und vielleicht sogar mal jemand herausnimmt aus dem Prozess, wenn man sieht, der ist schwer-sucht-erkrankt oder hat Rückfälle, dann muss man ihn sofort rausnehmen und therapieren« (Kommunaler SGB-II-Träger-Mitarbeiter, Interview04Regio06).

Die sozial aktivierenden Maßnahmen wirken bis in die einzelnen Lebensbereiche der Personen hinein. Sie liefern SGB-II- und Maßnahmenträger Einblicke in die unmittelbaren Lebensbedingungen und -umgebungen der Personen, beispielsweise über aufsuchende Dienste. Daneben fördern und fordern sie in scheinbar nicht investigativen Interaktionsstrukturen wie der gemeinsamen Mahlzeit, der offenen Beratung im Beratungsbus oder Sportkurs.

»Und davon halte ich eigentlich sehr viel, weil ich das Gefühl, dass es den Menschen einfach noch mal öffnet, also ganz, ganz weit öffnet und sie einfach in ihren Begabungen und 
Talenten unterstützt. Ohne das man ganz straight sagen kann, das führt auch unmittelbar zu der und der Einsatzstelle.« (Fachbereichsleiterin, Interview04Regio06)

Sozial aktivierende Maßnahmen haben das Vermögen, bis in die zeitweise sehr intensive Begleitung der Familie hineinzuwirken. Dies kann eine sehr hilfebedürftige Klientel auf einer sehr viel ganzheitlicheren Ebene auf dem Weg zur Erwerbsintegration begleiten: Potentiale erfassen, die zuvor nicht sichtbar waren sowie Hemmnisse aufdecken, die nur über den intensiven Austausch und ein gewonnenes Vertrauensverhältnis hervortreten. Es könnten Problemsituationen aufgedeckt werden, die aus den Familien- oder peer-group-Strukturen heraus entstehen und durch die Identifikation überhaupt erst bearbeitet werden können. Damit wird der Aktivierungsdiskurs besonders ernst genommen, indem das Individuum im Sinne des Enablings dabei unterstützt wird, sich selbst zu helfen, selbst aktiv zu sein, selbst sein eigenes Leben und die Aufnahme seiner Arbeitstätigkeit zu gestalten. Dies soll geschehen indem Wertschätzung, Selbstsicherheit und das Erlernen sozialer Kompetenzen als Vorstufe der Erwerbstätigkeit gefördert werden. Hier wird eine sehr unbürokratische und damit umfassend ansetzende Hilfe durch das Zusammenwirken verschiedenster Institutionen ermöglicht. In einem gewissen Widerspruch dazu steht die Tatsache, dass beispielsweise aufsuchende Dienste in den Kompetenzbereich des Allgemeinen Sozialdienstes gehören. So erklärte etwa eine Maßnahmenträgerleitung die Durchführung von aufsuchenden Diensten, die normalerweise nicht zum SGB-IIAufgabenspektrum gehören: »Soziale Integration ist immer grenzwertig. Die Verwaltungsvorschriften, die Gesetze so weit gut nach außen zu dehnen, dass es den Menschen hilft.« (Maßnahmenträgerleiterin, Interview03Regio04)

Daneben wird in diesen Maßnahmen, insbesondere im Hinblick auf eine jugendliche Klientel, in gewisser Weise ein erzieherischer Auftrag verfolgt und die normative Steuerung der Beschäftigungsförderung soweit möglich bis in die Wohnungen getragen: »Es wird wirklich ganz unten angefangen, mit dem Träger zusammen, dass aufzugreifen, was die Eltern zu Hause, ich sage jetzt mal 16 Jahre zu Hause versäumt wurde.« (Fallmanagerin, Interview02Regio04)

Doch auch die Aktivierung selbst kann als erzieherischer Akt und die Arbeitsverwaltung als sozialisierende Instanz betrachtet werden, wie Lessenich dies für den Sozialstaat insgesamt herausarbeitet (Lessenich 2012b). Der durch Maßnahmen Sozialer Aktivierung ermöglichte und meist über die Grenzen der Arbeitsverwaltung hinausreichende Einblick in das Lebensumfeld und die Persönlichkeit der SGB-II-Leistungsempfangenden, muss hierbei im Rahmen der Arbeitsverwaltung vertrauensvoll behandelt werden. Dennoch führt er nolens volens zu einer Ausweitung der über die Arbeitsverwaltung ausgeübten Bio-Macht 
in persönliche Bereiche. Die Bio-Macht (Foucault 1976) richtet sich auf die Bevölkerung und befasst sich auch mit der Regulierung ihrer Lebensweise. Durch die Übertragung auf die Arbeitsverwaltung werden Aspekte sichtbar, die ohne die Ausweitung in eine offenbar institutionsfreie Zone, etwa des gemeinsamen Essens oder des Kung-Fu-Kurses, nicht flagrant werden. Zudem transportiert die Sichtbarkeit der ganzen Persönlichkeit einen Handlungszwang zu Aktivierungsinterventionen der Beschäftigungsförderung in weitere Bereiche. Zum Beispiel müssen offen gelegte Suchtproblematiken registriert und behandelt werden. Wenn das Subjekt die Notwendigkeit einer Therapie jedoch (noch) nicht verinnerlicht hat, stoßen die Aktivierungsinterventionen an ihre Grenzen. Dienstleistungen Dritter, wie Suchtberatungsstellen, haben die interne Vorgabe, dann zu agieren, wenn die Klientin oder der Klient eine intrinsische Motivation zur Überwindung der Sucht haben. Denn andernfalls ist die Abbruchwahrscheinlichkeit der Therapie sehr hoch. Das Subjekt selbst wird durch Maßnahmen Sozialer Aktivierung in seiner ganzen Persönlichkeit in das Prinzip der Rechte und Pflichten eingebunden. Somit expandiert der Sanktionsvorbehalt mitunter in sehr private Bereiche, wie die Suchttherapie, Pflege des Äußeren oder Familienbeziehungen. Denn ein Individuum, das sich den Vorgaben entzieht, muss »natürlich auch mit den Konsequenzen [leben; CF], aber es hat alles einen erzieherischen Charakter.« (Fallmanagerin, Interview02Regio04)

Summa summarum zeigt sich, dass alle Maßnahmen Sozialer Aktivierung die Funktion einnehmen, die Beschäftigungsfähigkeit als Vorstufe der Erwerbsintegration zu fördern, wie dies die Aussage einer Teamleiterin illustriert: »Es ist nicht jeder Integrationsgedanke, den wir haben, Integration erster Arbeitsmarkt, sondern auch: Beschäftigungsfähigkeit steigern, verbessern und also die Integrationsfähigkeit für die Zukunft zu entwickeln, und das kann manchmal ein bisschen weiterer Weg sein.« (Interview01Regio05)

Letztlich zielt die Unterstützung der Akteurinnen und Akteure der Beschäftigungsförderung darauf, die Personen zur Eigeninitiative für die individuellen Vorbedingungen der Erwerbstätigkeit zu sensibilisieren und zu motivieren:

»Stabilisierung der Kunden, das ihnen bewusst wird meistens haben sie ja so ein Grundgefühl, irgendwas läuft nicht, aber sie wissen nicht so genau was, was ist es, wie identifiziere ich das und das passiert in der Maßnahme und dann werden auch die ersten Schritte gemacht, um diese Punkte zu lösen« (Fallmanagerin, Interview02Regio07).

Diese Vorstufe, auch wenn sozialarbeiterische Bezüge wie die Unterstützung bei psychischen, physischen, finanziellen oder anderen Problemlagen bestehen, ist 
im Kontext der Beschäftigungsförderung jedoch immer unter der Prämisse der (langfristigen) Erwerbsintegration angesiedelt und damit als Vorstufe der Erwerbstätigkeit auf den Aktivierungsdiskurs rückbezogen:

»Weil ich immer finde, [...] die Hinführung zum Arbeitsmarkt ist das A und O. Das [alle Formen sozial aktivierender Maßnahmen; CF] sind einfach Vehikel. [...] Aber das sind wichtige Vehikel und eben andere Ansätze, weil Frontalunterricht und Regelschule das kennen die [Maßnahmenteilnehmenden; $\mathrm{CF}$ « (Maßnahmenträgerleitung, Interview04Regio12).

Dieser Rückbezug auf die Erwerbsintegration ist einerseits durch die Ausrichtung der Bundesagentur für Arbeit und der SGB-II-Träger institutionell gegeben. Dies kann durch die rechtliche Reglementierung und Aufgabenteilung der Institutionen im Sozialstaat begründet werden. Neben diesem strukturellen Argumentationsgang ist es den AkteurInnen der Beschäftigungsförderung vor Ort ebenfalls ein Anliegen, die rechtskonforme Mittelverwendung zu verantworten:

»Man muss das irgendwie auch entsprechend darstellen und auch vertreten in der Öffentlichkeit, sonst könnte man den Eindruck kriegen, also Tai-Chi-Kurse für Arbeitslose. Also wir gehen hier arbeiten und die machen hier Bewegungstraining und Frühsport und alles auf Kosten des Steuerzahlers. [...] Man muss sie schon vorbereiten auf den Arbeitsmarkt auch und auf die Anforderungen, aber es wäre glaube ich nicht fair, wenn man nicht sehen würde, dass da gewisse Hindernisse im Verborgenen liegen und die sonst nicht bearbeitet werden.« (Kommunaler Mitarbeiter, der Sozialbetriebe betreut, Interview04Regio06)

So dient die Prämisse der Erwerbsintegration in der AkteurInnensicht auch dazu, die SGB-II-Leistungsbeziehenden anzuerkennen. Dementsprechend möchten so die Fachkräfte der Arbeitsverwaltung - die meisten Menschen arbeiten und würden sich nicht ernstgenommen fühlen, wenn einzig musische, sportliche oder kreative Fähigkeiten unterstützt würden. Zudem sei die ganzheitliche Förderung von Geist und Körper notwendig, da eine Unterstützung des Erwerbseinstiegs erst möglich sei, wenn die Menschen in ihrer Ganzheitlichkeit angesprochen würden.

Die Funktion der Förderung der Beschäftigungsfähigkeit verweist darauf, dass die Subjektdefinition des Aktivierungsdiskurses über >ihre` Kundinnen und Kunden bei der Klientel der Maßnahmen Sozialer Aktivierung nicht tragfähig ist. Denn die niederschwelligen Maßnahmen gehen einher mit umfangreichen Unterstützungs- und Beratungsleistungen, um in einer mehr oder minder weit entfernten Zukunft eigenverantwortliches und autonomes Handeln der SGB-II- 
Leistungsbeziehenden herzustellen. Sie seien erst dann mit einer größeren Eigeninitiative, dem Wissen zu Mitteln der Problembewältigung und dem Wunsch zur Erwerbsintegration ausgestattet. Die Klientel der Maßnahmen Sozialer Aktivierung gilt letztlich als unvollendetes Subjekt der Kundin respektive des Kunden ${ }^{89}$ im Sinne des Aktivierungsdiskurses. Das Potential der Maßnahmenteilnehmenden könne zwar erkannt, jedoch deren Realisierung erst nach einer Persönlichkeitsentwicklung gedacht werden.

Maßnahmen Sozialer Aktivierung haben, wie bereits entwickelt, die Eigenschaft der Dekommodifizierung. Während der Aktivierungsdiskurs die zu aktivierende Klientel der Arbeitsverwaltung auf sehr arbeitsmarktferne Personen, im Zuge der Umstrukturierung zur aktivierenden Arbeitsmarktpolitik, ausweitet und die sozialstaatliche Ausübung damit den Warencharakter der Arbeit weitet (Rekommodifizierung), ermöglichen die Maßnahmen Sozialer Aktivierung primär in langfristiger Perspektive eine Erwerbsintegration und wirken damit dekommodifizierend. Die Individuen werden zunächst zur Maßnahmenteilnahme verpflichtet und der Druck zu einer sofortigen Erwerbsaufnahme damit mittelfristig gemindert. Gerade Maßnahmenketten können für diese sehr arbeitsmarktferne Klientel eine legitime Sphäre der Teilhabe in der Erwerbsgesellschaft darstellen, in der sie der eingeforderten Gegenleistung nachkommen, selbst wenn sie keine Erwerbstätigkeit ausüben (können). Durch die Ausweitung des Maßnahmenportfolios von zeitnah integrierenden Maßnahmen auf primär nur in langfristiger Perspektive integrierende Maßnahmen wie die soziale Aktivierung, erhält der Aktivierungsdiskurs in seiner Praxis durch die Arbeitsverwaltung partiell einen dekommodifizierenden Charakter. Die Erwerbsaufnahme wird durch legitime Maßnahmenteilnahme(-n) verschoben und die (aktuelle) >Unfähigkeit` der Erwerbsaufnahme sozial abgefedert. Die Erwerbsfähigkeit zunächst aufzubauen, wird damit legitim.

Ferner wird die Teilhabe sehr arbeitsmarktferner Arbeitsloser über Maßnahmen Sozialer Aktivierung angestrebt. Dies ist ein Mittel, um Exklusionstendenzen aus der Lohnarbeitsgesellschaft zumindest teilweise entgegenzuwirken. Indem Arbeitslose eine Gegenleistung für die `beanspruchte ( Sozialleistung erbringen, können sie negative Zuschreibungen, die mit dem Erwerbsfähigkeitsstatus bei gleichzeitiger Arbeitslosigkeit verbunden sind (deserving poor), zumindest partiell widerlegen. In toto zeigt sich, dass die Arbeitsverwaltung in der Praxis das Thema der Sockelarbeitslosigkeit insofern bearbeitet, als passgenaue

89 Vergleiche Kapitel 4.2 »Menschen in Arbeit bringen«: Sicht auf die Leistungsbeziehenden. 
Instrumente die sehr arbeitsmarktferne Klientel aktivieren und die Beschäftigungsfähigkeit fördern sollen.

Zusammengefasst fungieren Maßnahmen Sozialer Aktivierung in ihren sozialstaatlichen Funktionen als Instrumente der Beschäftigungsförderung, die auf die ganze Persönlichkeit zielen und somit eine ganzheitliche Perspektive der arbeitsverwaltenden Förderung von Erwerbstätigkeit einnehmen. Darüber hinaus steht zunächst die Beschäftigungsfähigkeit als Vorstufe der Erwerbsintegration im Vordergrund. Durch diesen zeitlichen Aufschub der Arbeitsaufnahme fungiert die Maßnahme Sozialer Aktivierung als dekommodifizierendes Instrument, die den Warencharakter der Arbeit, zumindest vorübergehend, mindert. Ferner sind solche Maßnahmen im Aktivierungsdiskurs legitime Räume in denen Sozialleistungsempfangende ihren sozialen Status verbessern können, indem sie eine Gegenleistung (zunächst statt einem Erwerbseinstieg) erbringen. Letztlich ermöglichen solche Maßnahmen soziale Teilhabe. 



\section{Resümee und Ausblick}

Mit dieser Studie wurde das Phänomen der Maßnahmen Sozialer Aktivierung exploriert sowie dessen Entstehung und Funktionen analysiert. Maßgeblich für die neue Maßnahmenform ist eine Ausweitung des zu aktivierenden Personenkreises, die aus der Zusammenlegung von Sozial- und Arbeitslosenhilfe resultierte. Dadurch sollen nun auch sehr arbeitsmarktferne Personen aktiviert werden. Damit korrespondieren die untersuchten Maßnahmen, die sich dezidiert mit niederschwelligen, stabilisierenden und persönlichkeitsfördernden Elementen an eine sehr arbeitsmarktferne Klientel richten. Sie sind damit ein Spezifikum in der Beschäftigungsförderung, die im Kontext des politisch-rechtlichen Aktivierungsdiskurses agiert, der eigentlich auf eine zeitnahe Erwerbsintegration zielt. Im Folgenden werden die eingangs formulierten vier Forschungsfragen rekapitulierend beantwortet (Kapitel 6.1) und die Ergebnisse diskutiert (Kapitel 6.2).

\subsection{RekAPITULATION DER ForschungsfRAgEN}

\subsubsection{Typische Maßnahmenformen Sozialer Aktivierung}

1. Forschungsfrage (Kapitel 5.1):

Wie schlägt sich die Anforderung einer niederschwelligen und ganzheitlichen, nicht primär auf Erwerbstätigkeit gerichteten Maßnahme Sozialer Aktivierung in ihrer inhaltlichen Ausgestaltung nieder?

Inhaltlich beruhen Maßnahmen Sozialer Aktivierung auf Tätigkeiten, die eine weniger intensive Verknüpfung zum Arbeitsmarkt aufweisen. Der Fokus liegt weniger darauf, Schulabschlüsse, Weiterbildungen oder Praktika zu absolvieren. Dieses ist eher anschließenden Maßnahmen vorbehalten. Erste Kontakte mit einfachen, auch auf dem Arbeitsmarkt nachgefragten Tätigkeiten, werden hier meist 
weniger zeitintensiv und zielorientiert verfolgt. Denn der Klientel wird zugeschrieben, diese Schritte überhaupt erst nach Klärung weiterer Problemlagen unternehmen zu können.

So orientiert sich die inhaltliche Ausrichtung an lebenspraktischen, künstlerischen, musischen oder sportlichen Tätigkeiten. Von diesen nehmen die Befragten an, dass sie für die Klientel von Interesse sind. Daraus ergibt sich die vielgestaltige und innovative Ausgestaltung der Maßnahmen.

Durch die Maßnahmenträger-Fachkräfte sollen in den Maßnahmen Sozialer Aktivierung sogenannte Vermittlungshemmnisse aufgedeckt und gegebenenfalls Problemlagen über die Einbeziehung Dritter (Soziale Dienste, Beratungsstellen etc.) geklärt werden, etwa bei familiären Problemlagen oder Erkrankungen. Ein weiterer Schwerpunkt liegt bei sportlichen, künstlerischen und musischen Angeboten, die teilweise auf die Interessengebiete der Teilnehmenden ausgerichtet werden. In einigen Maßnahmen werden die Ergebnisse vor Publikum aufgeführt (etwa Tanz- oder Theaterproduktionen) oder publiziert (CD-Produktionen). Weiter sollen Alltagsstrukturen und Wissen um günstige Einkaufsoptionen respektive Reparaturmethoden für die Dinge des täglichen Bedarfs ausgebaut werden. Insgesamt stehen die sozial aktivierenden Maßnahmen inhaltlich unter dem Fokus, Lösungsstrategien für Problemlagen herauszuarbeiten sowie individuelle Stärken auf einer ganzheitlichen Ebene zu entwickeln, um zum Maßnahmenende oder in einer Folgemaßnahme dann an der Förderung der Erwerbsintegration ansetzen zu können.

\subsubsection{Genese der Maßnahmen Sozialer Aktivierung}

2. Forschungsfrage (Kapitel 5.2; 5.3):

Wie entstehen die niederschwellig ausgerichteten Maßnahmen Sozialer Aktivierung im Umfeld eines auf kurzfristige Arbeitsmarktintegration ausgerichteten politisch-rechtlichen Diskurses?

Die Schaffung von beschäftigungsfördernden Maßnahmen findet in einem komplexen Regelwerk aus rechtlichen und institutionellen Vorgaben sowie den Rahmenbedingungen des Finanzbudgets statt. Maßnahmen Sozialer Aktivierung wurden durch Maßnahmen- sowie SGB-II-Träger initiiert und zum Teil über Regionale Einkaufszentren beschafft. Die unterschiedlichen Entstehungswege sowie die gewählten rechtlichen Realisierungsformen bergen divergierende Grade der Kommunikationsintensität zwischen Maßnahmen- und SGB-II-Trägern sowie unterschiedliche Ausmaße der Maßnahmenflexibilität. 
Sozial aktivierende Elemente sind insbesondere vor dem Jahr 2010 noch in umfangreichere Maßnahmen eingebunden, in denen meist auch qualifizierende oder ausbildende Module enthalten sind. Häufig sind sozial aktivierende Elemente dann in Arbeitsgelegenheiten integriert, in denen gemeinnützige und zusätzliche Tätigkeiten verrichtet werden. Erst mit der Schaffung des Aktivcenters ${ }^{1}$ im September 2010 können niederschwellige und sozialstabilisierende Instrumente auch mit einer stärkeren Fokussierung auf diese Aspekte als Standardinstrumente geschaffen werden. Hier werden Schlüsselqualifikationen und eine Gesundheitsorientierung als integraler Bestandteil des bundesweiten Standardinstruments auch mit Teilzeitmaßnahmen-Optionen geschaffen, um eine sehr niederschwellige Maßnahme ins Portfolio der Beschäftigungsförderung aufzunehmen. Dem Aktivcenter kommt insofern eine herausragende Bedeutung zu, als es bottom-up durch die intensive Nachfrage der SGB-II-Träger entstanden ist. Die politischen Richtlinien des Bundesministeriums für Arbeit und Soziales sowie die Steuerung der Bundesagentur für Arbeit kamen durch die Bereitstellung des Standardinstruments letztlich dem Wunsch der SGB-II-Träger-Fachkräfte nach, Instrumente für eine sehr arbeitsmarktferne Klientel bereitzustellen. So verweist die Implementierung des Aktivcenters auf eine von den SGB-II-Trägern wahrgenommene Notwendigkeit von niederschwelligen Maßnahmen, die eine spezifische Klientel sozial aktivieren muss, um sie langfristig in Erwerbstätigkeit integrieren zu können.

Diese neuartige Maßnahmenform wurde allerdings in der Arbeitsverwaltung und dem öffentlichen Diskurs zumeist nicht dezidiert herausgestellt, da sie nicht über ihre inhaltliche, sondern über ihre rechtliche Realisierungsform im Controlling erfasst wurde. Das, die Maßnahmenform konstituierende, Attribut der Persönlichkeitsstabilisierung wird durch die dort bestehende Kategorisierung nicht abgebildet. Umso entscheidender ist es, dass sich soziale Aktivierung trotz der starken Orientierung an Vermittlungsquoten in den ersten Arbeitsmarkt in einem hierarchischen Maßnahmenentstehungsfeld herausbilden konnte. ${ }^{2}$

1 Nach § 16 I SGB II i.V.m. § 46 I S. 1 SGB III und ab April 2012 verankert im § 16 Abs. 1 SGB II i.V.m. § 45 SGB III.

2 Das quantitative Vorkommen von Maßnahmen Sozialer Aktivierung wurde in der vorliegenden qualitativen Studie nicht erfasst, sie bildet vielmehr eine fundierte Basis durch die Kenntnis von Genese- und Realisierungsformen, um quantitative Studien anschließen zu können. 


\subsubsection{Spannungsfelder im Maßnahmenkontext}

3. Forschungsfrage (Kapitel 5.4):

Welche Themen treten in dem zwangsläufig entstehenden Spannungsfeld zwischen dem Aktivierungsdiskurs und der Praxis der Arbeitsverwaltung zu tage?

Mit der praktizierten Implementierung von Maßnahmen Sozialer Aktivierung geraten der Einfluss und die Gewichtung folgender zwei Ausrichtungen in den Blick: 1.) die Aktivierung der Klientel oder 2.) der möglichst uneingeschränkte Wettbewerb zwischen den Maßnahmenträgern. Ein hoher Grad an Autonomie in der Planung und Durchführung von Maßnahmen Sozialer Aktivierung kann nach Erfahrung der SGB-II- und Maßnahmenträger-Fachkräfte dazu führen, flexibel auf die sehr arbeitsmarktferne und teilweise unbekannte (da trotz Sanktionierung nicht vorstellige Klientel) reagieren zu können. Hier werden positive Aspekte eines intensiven Austausches zwischen SGB-II- und Maßnahmenträger gesehen. Dies kann jedoch zu Wettbewerbsverzerrungen führen.

Zur uneingeschränkten Gewährleistung des Wettbewerbs im Beschaffungsprozess dienen die Regionalen Einkaufszentren. Sie stellen einerseits die Rechtskonformität insbesondere in einem komplexen rechtlichen Rahmen her. Andererseits zeigt sich der Maßnahmenkauf verstärkt bürokratisiert, sowohl über eine forcierte Formalisierung in den Verfahrensabläufen als auch in den Maßnahmeninhalten. Innovative Maßnahmen müssen hier zur Realisierung größere Hürden überwinden. Insbesondere niederschwellige Maßnahmen, die auf eine langfristig angelegte Erwerbsintegration einer arbeitsmarktfernen Klientel zielen, sind strukturell benachteiligt (bis ein Standardinstrument existiert), da auf eine zeitnahe Arbeitsmarktintegration zielende Kennzeichen maßgeblich sind.

Obwohl durch die Schaffung eines Standardinstruments - dem Aktivcenter auch über das Regionale Einkaufszentrum Maßnahmen Sozialer Aktivierung unkompliziert beschafft werden können, ist der Handlungsraum der AkteurInnen beschränkt. Denn die Budgetierung setzt Grenzen unabhängig von einer REZHinzuziehung, indem ein Großteil der Ressourcen für beschäftigungsfördernde Instrumente eingesetzt werden muss, um die Eingliederungsquoten zu erfüllen. Diese Vorgaben begünstigen kurzfristig in den Arbeitsmarkt integrierende Instrumente, die besonders für eine arbeitsmarktnahe Klientel als passend gelten. So können die niederschwellig angelegten Maßnahmen, die eher langfristig Eingliederungsquoten erhöhen, vor allem dann durchgeführt werden, wenn die Integrationsquoten bereits übererfüllt sind und das Integrationsbudget noch nicht ausgeschöpft. Diese Rahmenbedingungen befördern den Creaming-Effekt, dem- 
zufolge insbesondere arbeitsmarktnahe Personen in der Arbeitsvermittlung aktiviert und dabei unterstützungsintensivere Gruppen weniger berücksichtigt werden.

So ergibt sich insgesamt ein Spannungsfeld zwischen den top-down wirkenden Vorgaben, die auf eine zeitnahe Erwerbsintegration zielen, und dem Bottomup-Bestreben der Praxis der Arbeitsverwaltung, arbeitsmarktferne Personen über längerfristig wirkende Instrumente zu integrieren. Daher sind das Vorkommen und die Entstehung von Maßnahmen Sozialer Aktivierung bemerkenswert, denn sie zeugen von der letztlichen Durchsetzung eines bottom-up implementierten niederschwelligen Standardinstruments (dem Aktivcenter). Ferner können diese niederschwelligen und sozialstabilisierenden Maßnahmen der wissenschaftlichen Diagnose einer neoliberale Wende (Butterwegge 2006) beziehungsweise der Durchsetzung eines liberalen Wohlfahrtsregimes (Münch 2009) partiell entgegenwirken. Denn gleichwohl eine starke Arbeitsmarktorientierung in das SGB II eingeschrieben ist, entstehen im Praxisfeld der Arbeitsverwaltung eher sozialpolitische und an dem Enabling-Konzept orientierte Maßnahmen und Maßnahmenbestandteile, die die Persönlichkeitsstabilisierung und soziale Teilhabe fördern sollen. Gleichzeitig können Maßnahmen Sozialer Aktivierung jedoch auch als ganzheitlicheres Instrument dienen, um die von Lessenich konstatierte sozialstaatliche Aufgabe zur »marktgerechten Selbsterziehung der Leute und damit zur sozialen Praxis des alltäglich-subjektiven doing capitalism « (Lessenich 2012b: 61) bei arbeitsmarktfernen Personen anzuwenden.

\subsubsection{Funktionen der Maßnahmen Sozialer Aktivierung}

4. Forschungsfrage 4 (Kapitel 5.5):

Welche Funktionen nehmen Maßnahmen Sozialer Aktivierung ein, wenn sie hinsichtlich des zugeschriebenen Interventionsziels, Subjektkonstitution, Tätigkeitsform und Aktivierungsform untersucht werden?

Die Funktionen der Maßnahmen Sozialer Aktivierung konnten auf drei Ebenen rekonstruiert werden: 1.) den institutionenspezifischen, 2.) den sozialen und 3.) den sozialstaatlichen Funktionen.

Unter den institutionenspezifischen Funktionen kommt aus Perspektive der Bundesagentur für Arbeit den Maßnahmen hauptsächlich als Clearinginstrument eine große Bedeutung zu. In diesem Rahmen wird geklärt, ob die zu aktivierenden Personen als erwerbsfähig, und damit im SGB II verortbar erscheinen. Weiter ist die Aktivierung der erwerbslosen Leistungsempfangenden beabsichtigt, da insgesamt der Charakter einer Gegenleistung für Sozialleistungen transportiert 
werden soll (aktive statt passive Leistungen). Ebenfalls für die SGB-II-Träger sind das Clearing sowie die Aktivierung von Bedeutung. Darüber hinaus ist jedoch die Installierung eines sozialen Netzwerkes um arbeitsmarktferne Personen mit mehreren Problemlagen wesentlich. Über dieses Netzwerk soll die Entwicklung von Potentialen, die Bearbeitung der Problemlagen und letztlich die Arbeitsmarktintegration forciert werden. Daneben sind diese Maßnahmen ein Instrument, um dem Unterstützungsanliegen von SGB-II-Träger-Fachkräften nachzukommen, das diese bezüglich der arbeitsmarktfernen Klientel äußern. Für die durchführenden Maßnahmenträger ist auf der institutionenspezifischen Ebene insbesondere der Erwerb von Drittmitteln essenziell. Über unterschiedliche Geldgeber und Konditionen können mehrere Finanzierungsfelder erschlossen werden. Weiter ermöglichen Maßnahmen Sozialer Aktivierung vergleichsweise längere Maßnahmendauern und somit längere Planungshorizonte. Außerdem steigen die Maßnahmenträger bei Erfahrung und speziellen Angeboten für arbeitsmarktferne Leistungsbeziehende in ihrer Attraktivität gegenüber den SGBII-Trägern, die diese Klientel aktivieren möchten. Darüber hinaus wird den Maßnahmen eine motivationssteigernde und damit die Maßnahmenabbrüche von Teilnehmenden reduzierende Wirkung zugesprochen. Für ein tiefergehendes Verständnis des organisationalen Kontextes und der weiteren Bedingungsfaktoren wären weitere Studien mit der Einbeziehung von Verlaufsdaten erkenntnisträchtig.

Die sozialen Funktionen konnten über die Rekonstruktion verschiedener Typen der Aktivierung beschrieben werden. Anhand dieser werden spezifische Aktivierungs- sowie soziale Integrationsformen deutlich. Insgesamt werden die Personen in normative Strukturen integriert, die einerseits durch legitime Wissensformen, aber auch durch AkteurInnen und Institutionen wie die Arbeitsverwaltung oder Sozialwirtschaft moduliert werden - in der vorliegenden Untersuchung als Ordnungen bezeichnet. Diese Ordnungen wirken auf die Alltagsstrukturen der Maßnahmenteilnehmenden ein, indem Tagesabläufe simuliert und für die Erwerbstätigkeit notwendige Voraussetzungen vorbereitet werden. Die Funktion der Integration in die Ordnung der Tätigkeit intendiert Arbeitstugenden über unterstützende Aufgabenbereiche, Dienstleistungen und Produkte der Subsistenz herzustellen. Die Integration in die Ordnung der erwerbstätigen Familie bearbeitet Familienproblematiken und Rahmenbedingungen für die Erwerbstätigkeit über die Simulation von Erwerb. Die Integration in die Ordnung der Arbeitsverwaltung stellt Kontakt zu einer zuvor unerreichbaren Klientel über neuartige Kommunikationswege her und kann so Vertrauen aufbauen, bei wachsendem latenten Kontrollpotential. Die Integration in die Ordnung des gesellschaftlichen Miteinanders aktiviert über Gruppenprozesse, um sozialkompetentes Handeln zu 
fördern und deviantes Auftreten zu mindern. Die Integration in die Ordnung des aktiven Subjekts aktiviert zur Selbstwirksamkeit, um die individuelle Motivation zur Erwerbstätigkeit herzustellen. Die Ordnung der Tätigkeit sowie die Ordnung der erwerbstätigen Familie stehen dabei dem Aktivierungsdiskurs am nächsten, wenn sie auch die Erwerbsintegration selbst kaum erreichen, während die anderen Ordnungen eher die unterstützenden und sozialarbeiterischen Elemente betonen. Insgesamt werden die Maßnahmenteilnehmenden in Alltags- und Zeitstrukturen sowie soziale Interaktionsfelder eingeführt, die nicht nur für die Maßnahmenteilnahme, sondern auch für die Erwerbstätigkeit als bedeutsam betrachtet werden. Doch die Typen stehen in ihrer unmittelbaren Ausrichtung konträr zum Aktivierungsdiskurs, da eine kurzfristige Erwerbsintegration, die im Sinne des Controllings messbar ist, nur höchst selten erfolgen kann beziehungsweise zunächst nicht intendiert wird. Es wird jedoch eine erweiterte Aktivierungsform gebildet, die eine teilweise Umdeutung des Diskurses enthält. Denn selbst wenn die Erwerbsintegration nicht gelingt, könne doch eine erfolgreiche Aktivierung verzeichnet werden.

Die sozialstaatlichen Funktionen der Maßnahmen beinhalten vorrangig die Ganzheitlichkeit. Die Teilnehmenden werden in ihrer ganzen Person analysiert und unterstützt, jedoch gleichzeitig problemlösendes Handeln der Personen auf vielfältigeren Ebenen kontrolliert als dies beispielsweise bei Bildungsmaßnahmen der Fall ist, und erlauben es, der Arbeitsverwaltung, bis in die privaten Lebensbereiche der Personen zu wirken. Die Subjektdefinition des Aktivierungsdiskurses greife in der AkteurInnensicht, nicht bei der Klientel der Maßnahmen Sozialer Aktivierung. Vielmehr können die Individuen als unvollendete Subjekte der Kundinnen und Kunden verstanden werden. Denn obwohl sie das Potential in sich tragen, vermögen sie frühestens während oder nach einer geleisteten Unterstützung in Form einer sozial aktivierenden Maßnahme autonom zu Handeln. So zielen die Maßnahmen auf die Beschäftigungsfähigkeit, die als Vorstufe der Erwerbsintegration gefördert wird und damit eine langfristige Perspektive auf die eigentliche Zielsetzung des Aktivierungsdiskurses einnimmt. Die Problembearbeitung wird zum legitimen Schritt vor der Erwerbsintegration und der Druck zur Erwerbsaufnahme zunächst gemindert. Dadurch fungieren Maßnahmen Sozialer Aktivierung als dekommodifizierende Instrumente, der ansonsten rekommodifizierenden Ausrichtung des Aktivierungsdiskurses. So wird das Individualisierungsprinzip aufgeweicht und die Eigenverantwortung zumindest temporär durch eine teilweise umfangreiche Unterstützung ersetzt, um Problemlagen im Sinne des Enablings zu bearbeiten. Denn die Hilfestellung dient dazu, dass sich die Individuen künftig selbst helfen. Ferner erbringen die Maßnahmenteilnehmenden eine im Sinne des Aktivierungsdiskurses erwünschte Gegenleis- 
tung für Sozialleistungen. So kann negativen Zuschreibungen (deserving poor) partiell entgegengewirkt werden, da die (aktuelle) Nicht-Erwerbstätigkeit in den legitimen Status der Maßnahmenteilnahme übergeht. Dadurch wird eine Rehabilitierung der Maßnahmenteilnehmenden in ihrer sozialen Position erreichbar sowie Teilhabemöglichkeiten in der Maßnahme selbst bereitgestellt.

\subsection{Diskussion}

Zusammenfassend wird auch in Maßnahmen Sozialer Aktivierung auf das diskursiv gesetzte Handlungsproblem des Leistungsbezugs von sogenannten erwerbsfähigen Hilfebedürftigen reagiert. Doch offenbart die Genese der Maßnahmen Sozialer Aktivierung ein Gestaltungspotential der SGB-II-Träger-Fachkräfte, die bottom-up die Standardisierung des neuartigen Instruments Aktivcenter fördern konnten. Die diskursiv-kommunikativen Praktiken der SprecherInnen des Diskurses werden dementsprechend nicht bloß reproduziert, sondern auch verändert. Als Genesebedingungen von sozialer Aktivierung werden vor allem Anforderungen einer sehr arbeitsmarktfernen Klientel beschrieben. Das Subjektbild der AkteurInnen der Arbeitsverwaltung divergiert jedoch maßgeblich von der Subjektposition des Aktivierungsdiskurses als Kundin und Kunde. Dennoch wird eine Aktivierung vollzogen und somit in die Wissenspolitik ein ausgeweiteter Aktivierungsbegriff inkludiert. Hier vollziehen sich Anpassungsprozesse von zwei Seiten: 1.) der AkteurInnen der Arbeitsverwaltung an die Anforderungen der SGB-II-Leistungsbeziehenden sowie 2.) des Aktivierungsdiskurses an die Sichtweisen der AkteurInnen. Die AkteurInnen verändern so dispositive Elemente im Aktivierungsdiskurs. Die Analyse zeigt jedoch, dass weitere Fragen der sozialen Teilhabe zu stellen sind. Die hier konzentriert dargebotenen Thesen und Schlussfolgerungen werden nun näher ausgeführt.

Der Genesekontext der Maßnahmen Sozialer Aktivierung zeigt auf, dass diese zunächst über offene Instrumente eingeführt wurden. Das Spannungsfeld aus Top-down-Vorgaben und Bottom-up-Zielen der Aktivierung sowie Integrationsförderung wurde überwunden, indem das Standardinstrument des Aktivcenters geschaffen sowie das diskursive Konzept von Aktivierung in der Praxis der Arbeitsverwaltung ausgeweitet wurde. Die fortwährende Nachfrage durch die SGB-II-Träger-Fachkräfte hat diese Implementierung ausgelöst. Das neue Instrument richtet sich dezidiert an eine sehr arbeitsmarktferne Klientel und zeugt damit von einem Personenkreis im SGB-II-Bezug, der nach Ansicht der SGB-IITräger-Fachkräfte nicht mit herkömmlichen beschäftigungspolitischen Maßnahmen aktiviert werden kann, jedoch durchaus erreicht werden muss: »Und 
wenn wir die Leute jetzt nicht anpacken, dann fehlen uns die später in der Wirtschaft« (zentraler Projektmanager, Interview03Regio07). Dafür sollen die Personen psychisch und lebenspraktisch stabilisiert werden, etwa über Theatergruppen, Musikprojekte, Erweiterung des individuellen Bewegungsraumes durch kulturelle Veranstaltungen und Exkursionen. Mit dem Aufbau der Selbstwirksamkeit beispielsweise, sollen Ängste, Perspektivlosigkeit und Unsicherheit über die berufliche Zukunft reduziert werden, um anschließend die Erfolge in den arbeitsmarktfernen Bereichen auf den Arbeitsmarkt zu attribuieren. Wenn eine Theaterrolle geglückt und mit Ausdruck auf der Bühne gespielt wurde, soll das Wissen über Körperhaltung, Stimmeinsatz und Emotionskontrolle auch in einem Bewerbungsgespräch oder späteren Berufstätigkeiten unterstützen. Die eingeübten Kompetenzen beeinflussen letztlich die weichen Faktoren des Arbeitsmarktzugangs wie Präsentationskompetenz, räumliche Flexibilität oder Zeitmanagement. Zudem gehen Maßnahmen Sozialer Aktivierung mit der erweiterten Sichtbarkeit einer arbeitsmarktfernen Klientel sowie der Bearbeitung ihrer Problemlangen einher. Die neuartigen Maßnahmen allein könnten den notwendigen Handlungsbedarf der Arbeitsmarktverwaltung für diese Klientel jedoch nicht gewährleisten. So wird von den AkteurInnen der Arbeitsverwaltung vielfach nachdrücklich vertreten, dass mittel- bis langfristige Konzepte für die Förderung der sehr arbeitsmarktfernen Klientel notwendig seien. Denn neben der Verfügbarkeit von Maßnahmen Sozialer Aktivierung müssten auch die Rahmenbedingungen für ihre Durchführung verbessert werden: etwa die Finanzmittel sowie eine erweiterte Qualitätsdefinition und -messung im Controlling, die sich nicht überwiegend an der Erwerbsintegration orientiert. In dieser Studie zeigt sich bei den AkteurInnen der SGB-II-Träger, die mit den Maßnahmen der Sozialen Aktivierung betraut waren, überwiegend eine ganzheitliche, sozialintegrative sowie häufig sozialpädagogische Orientierung, die jedoch immer mit dem Fernziel der Erwerbsintegration konzipiert wurde. Dies nivelliert teilweise die beschriebenen konträren Verwaltungsphilosophien der zugelassenen kommunalen Träger (zkT, auch Optionskommunen) und den aus den Arbeitsgemeinschaften hervorgegangenen SGB-II-Trägern. Erstere galten als eher sozialintegrativ, risikofreudig und weniger hierarchisch, dagegen letztere als regelorientiert und auf spezielle Verwaltungsvollzüge ausgerichtet (Bieback 2009, Fachhochschule Frankfurt am Main/infas-Institut für Angewandte Sozialwissenschaft $\mathrm{GmbH} /$ Wissenschaftszentrum Berlin 2009, Dann et al. 2007). Unabhängig von der institutionellen Beschaffenheit findet sich häufig bei den befragten AkteurInnen der Arbeitsverwaltung ein ausgeprägtes Ethos zur Aktivierung sehr arbeitsmarktferner Personen: 
»dass man jeden aus der Gesellschaft versucht noch mitzunehmen. Das sage ich ganz vorsichtig, weil es gibt natürlich auch Leute, die wollen sich nicht mitnehmen lassen [...] Die Leute, da muss der Staat sagen und das sagt er ja auch, man kann sanktionieren, aber auch nur bis zu einem gewissen Maße [...], was ich aber wirklich erlebe [...] ist, dass wenn man die Menschen wirklich anpackt und motiviert eben viele der scheinbar unmotivierbaren Leute, dennoch motivierbar sind. Es kommt darauf an, dass man die ernst nimmt, denen zuhört, nicht nur oberflächliche Hilfen anbietet, nicht nur kurzfristig Hilfen anbietet, weil die Leute demoralisiert sind beziehungsweise nie richtig gelernt haben auch Hilfen richtig zu nutzen und sich selber wertzuschätzen und was aus ihrem Leben zu machen und das geht nicht in drei Monaten.« (Zentraler Projektmanager, Interview03Regio07)

Die vielfach vorgebrachte Notwendigkeit der gezielten Förderung dieser Klientel im SGB-II-Bezug aufgrund der vielfältigen Problemlagen (Kaltenborn/Kaps 2013, Schubert et al. 2013, Schütz et al. 2011, Oschmiansky 2010, Henkel et al. 2009, Ames 2008) wird durch Maßnahmen Sozialer Aktivierung vollzogen. Die Förderung der sozialen Aktivierung könnte dem Umstand entgegenwirken, dass sich der Zeit- und Mitteleinsatz in der Vermittlungspraxis zu Gunsten der arbeitsmarktnahen und $\mathrm{zu}$ Lasten der arbeitsmarktfernen Klientel verlagert hat (zum Beispiel Baethge-Kinsky et al. 2007: 62f.). Auch für 2014 prognostizierte Hindernisse zum Abbau von Arbeitslosigkeit, wie Suchtfriktionen (Fuchs et al. 2013), könnten durch sozial aktivierende Instrumente bearbeitet werden. In dieser Studie berichten die AkteurInnen der Arbeitsverwaltungspraxis, unter Bezugnahme auf die umfangreichen Problemlagen der sehr arbeitsmarktfernen Klientel, häufig von Kompetenzen, die sie benötigen würden, jedoch nicht in ihrer Ausbildung erhalten hätten. So wird von ihnen vielfach eine gezielte Schulung zur Beratung der Personen mit solchen mannigfaltigen Problemlagen gewünscht respektive reklamiert. Größere Zeitkontingente und niedrigere Fallzahlen werden ebenfalls gefordert. Auch in weiteren Studien wird von den Befragten auf die Notwendigkeit von gezielter Qualifizierung hingewiesen, etwa werden professionelle Fehler durch unterlassene Nachfragen zu Beratungs- und Unterstützungsbedarfen und Verweise auf Dienstleistungen Dritter erwähnt (Schütz et al. 2011, Tisch 2010, Baethge-Kinsky et al. 2007, Bieback 2009). Gerade das für intensive Betreuungsleistungen installierte Fallmanagement, das agieren soll, wenn eine Eingliederung in den ersten Arbeitsmarkt wegen Sucht, Krankheiten oder sozialen Problemen in den ersten sechs Monaten nicht wahrscheinlich ist (BMWA (ehemaliges) 2005a: 60), benötigt eine hinreichende quantitative und qualitative Ausstattung der direkt mit den SGB-II-Leistungsempfangenden interagierenden Fachkräfte. Hier besteht nach Kolbe, der Beratungsgespräche im SGB II empirisch untersucht, noch Handlungsbedarf: »Aktivierung als eine Ermächtigung zur 
Bearbeitung schwieriger Lebenssituationen ist mit den Fallbearbeitungen für die Hilfeberechtigten selten verbunden. In den Beratungen findet diese teilweise instrumentell zweckgebunden an das Ziel der Integration in den Arbeitsmarkt statt.« (Kolbe 2012: 204)

Kontakte zu sozialen Diensten und Unternehmen aufbauen gehört, neben der Arbeitsvermittlung und Beratung, häufig in das Aufgabengebiet des Fallmanagements: ${ }^{3}$

»Es ist ja mit in unserem Bereich, diese Netzwerke zu knüpfen, es ist natürlich, ich hab jetzt knapp 300 Kunden, es ist schwierig, aber es lohnt sich, weil man nachher davon so viele Vorteile hat. Ich kann dann im Gespräch auch besser erklären, der Kunde geht hin, das ist ja für mich auch ein Fortschritt, wenn die Schulden reguliert sind.« (Fallmanagerin, Interview02Regio07)

Andere Befragte sehen eine Option darin, direkt auf Beratungsstellen im Hause verweisen zu können, indem verstärkt Netzwerke im Gebäude der SGB-IITräger angesiedelt werden. ${ }^{4}$ Insgesamt zeigt sich, dass die Genese der Maßnahmenform auch ein Ausdruck des Verlagerns nach Problemlagenbearbeitung ist, die nicht direkt den Arbeitsmarkt betrifft. Denn häufig fungiert bei Maßnahmen Sozialer Aktivierung der Maßnahmenträger als Schaltstelle für das soziale Netzwerk. Von Nachteil können dabei die stark variierenden lokalpolitischen Einbindungen der Maßnahmenträger, der kurze Zeithorizont der Maßnahmen und die Möglichkeit, bei der nächsten Ausschreibung einem besseren Angebot zu unterliegen sein. Kooperationen verschiedener Leistungsträger, unter Ägide der SGB-II-Träger sind weder dem kurzen Projektzeitraum, noch der Ungewissheit der nächsten Ausschreibungsrunde ausgesetzt. Das Fallbeispiel einer Maßnahme, die psychisch-physische Unterstützungsangebote sowie gesundheitsorientierte Beschäftigungsvermittlung betreibt (Kapitel 5.1.4). legt offen, wie Jobcenter, Krankenkassen und Beratungsstellen ein gemeinsames, mittelfristig stabiles Netzwerk bilden.

Das Subjektbild des Aktivierungsdiskurses beschreibt autonome Subjekte Kundinnen und Kunden. In Anlehnung, respektive Abgrenzung, zu diesem Subjektbild finden Subjektivierungs- und Objektivierungsprozesse über Disziplinierung, Normierung und Sanktionen im Foucaultschen Sinne statt, die das Individuum erst zum Subjekt gerieren lassen (Foucault 1994: 236). In der Subjektposi-

3 So zum Beispiel in Interview02Regio07 und Interview03Regio11 sehr betont.

4 Etwa in Interview01Regio06, Interview02Regio05 und Interview01Regio10. 
tion wird eine Neudefinition staatlicher sowie bürgerlicher Aufgaben deutlich. Vom Aktivierungsdiskurs als Tauschverhältnis verstanden, kann die aktivierte Bürgerschaft unter Gegenleistungen Sozialleistungen erhalten. Die Autonomie der erwerbslosen Leistungsempfangenden drückt sich jedoch eher in einer Übernahme von Eigenverantwortung, denn in einem autonomen Handeln aus (Globisch 2012). Auch da die Autonomie sich insbesondere an betriebswirtschaftlichen Effizienzkriterien ausrichtet und selbstregulatorische Kapazitäten als individuelle »Technologien des Selbst« fördern soll (Bröckling et al. 2000). So wird das Individuum als Unternehmer seiner Selbst (Bröckling 2007) immer stärker in seiner Selbstverantwortlichkeit adressiert. Damit wird es zu einem Individuum, das mittels Selbsttechniken seine Marktfähigkeit fortwährend optimiert, die eigene Arbeit, Fähigkeiten und Zeit effizient organisiert, um sich unternehmerisch gekonnt auf dem Arbeitsmarkt und erfolgreich im Wettbewerb zu platzieren. Sogar wenn Individuen auf die unternehmerische Anrufung unterschiedlich reagieren, als Enthusiasten, Ironiker oder Melancholiker, so ist doch allen gemein, dass sie sich, um sich von anderen im Wettbewerb zu unterscheiden, beständig als Unternehmerisches Selbst inszenieren und re-inszenieren müssen (Bröckling 2012). ${ }^{5}$ Doch muss sowohl die ungleiche Verteilung der Ressourcen und damit die Bedingungen für die Selbstverantwortlichkeit für die flexiblen Individuen als auch die zunehmende Unsicherheit der Arbeitsverhältnisse berücksichtigt werden (Sennett 2000, Castel 2000, 2011). So erfordern die Arbeitsbedingungen in immer stärkerem Maße Beschäftigte als Arbeitskraftunternehmer (Pongratz/Voß 2003, 2004). Vor diesem Hintergrund werden in der Praxis der Arbeitsverwaltung Grenzen des aktiven Subjektbildes gesehen, die hier unter dem Begriff des unvollendeten Subjektes der Kundinnen und Kunden gefasst wurden. Denn die Anpassung der Bevölkerung an ökonomisches Handeln benötigt, in der Logik der AkteurInnen, andere Instrumente. Schließlich zeichnen sich die Teilnehmenden der Maßnahmen Sozialer Aktivierung durch einen umfassenden Unterstützungsbedarf bei der Bewältigung mannigfaltiger Problemlagen aus und sind in der Wahrnehmung der AkteurInnen noch nicht zu dem autonomen Handeln fähig, dessen es im Sinne des Aktivierungsdiskurses bedürfe:

»Im aktivierenden, präventiven Sozialstaat wird die Gesellschaft zum Bezugspunkt des Sozialen, wird die Subjektivität der Bürgerinnen und Bürger konsequent sozialisiert, für

5 Verworfenheiten der Individuen werden auch von Lutz Eichler aufgezeigt, der strategische Anerkennung als Urheber für narzisstische Interaktionsformen und Psychodynamiken beschreibt (Eichler 2013). 
soziale Ziele in Anspruch von sozialen Zwecken in Beschlag genommen, im Geiste des Sozialen programmiert. In diesem Sozialstaat werden die Subjekte am Grad ihrer Gesellschaftlichkeit, an der Erfüllung ihrer sindividuellen Pflicht` zum verantwortlichen Umgang mit den gemeinsamen Ressourcen gemessen. [...] Es gilt daher immer wieder [...], den Einzelnen als aktives, vorsorgendes, selbstverantwortliches Subjekt der Gesellschaft zuzuführen.« (Lessenich 2009b: 122)

So intendieren die interviewten SGB-II-Träger-Fachkräfte, sehr arbeitsmarktferne Leistungsbeziehende zu aktivieren und deren Problemlagen zu bearbeiten, um über die Unterstützung ein Enabling zu ermöglichen. Insofern wird einem durch den Aktivierungsdiskurs gesetzten Handlungsproblem begegnet - der Erhalt passiver Leistungen. Wenngleich keine Erwerbstätigkeit aufgenommen wird, so wird doch eine Gegenleistung für den Leistungsbezug und damit der Status >aktiviert` ermöglicht. Mit dieser zusätzlichen, zulässigen Option wird zugleich eine ausgeweitete Definition von Aktivierung in die Wissenspolitik inkludiert. Dies wird in der Praxis genauso mit der Intention der Erwerbsintegration verbunden, doch wird hier zunächst die Beschäftigungsfähigkeit gefördert, um einen langfristigen Erwerbseinstieg zu unterstützen. In dieser Studie wurde die AkteurInnensicht auf die Maßnahmenteilnehmenden exploriert. Eine darauf aufbauende vergleichende Studie mit Blick auf die sozial zu aktivierenden Maßnahmenteilnehmenden könnte genauere Auskünfte über die Fähigkeiten und Problemlagen der Personen bieten. Wenngleich in der vorliegenden Studie ein recht einheitliches Bild einer Klientel mit massiven physischen, psychischen und sozialen Beeinträchtigungen aufgezeigt wurde, könnten hier auch die Kategorisierungen in Kundenprofile der SGB-II-Träger-Fachkräfte dieses Bild unterstützen und reproduzieren. So zeigen Kratz für die Arbeitsverwaltung und Plankensteiner für die Jugendhilfe eine Fokussierung der Fachkräfte auf negative Aspekte der Erwerbsbiographien der Leistungsempfangenden, die wiederum die Eigenaktivität behindern (Kratz 2013a, Plankensteiner 2013). Insofern sind Maßnahmen Sozialer Aktivierung in der Förderung der Beschäftigungsfähigkeit durch ihren verglichen mit traditionellen Maßnahmen, ganzheitlicheren Fokus ein janusgesichtiges Phänomen: einerseits können sie in einem umfassenderen Sinne Enabling fördern und bei der Bewältigung von Problemlagen unterstützen, andererseits haben sie eine paternalistische Seite, indem sie tiefere Einblicke in die Alltagswelt der Teilnehmenden gewähren und umfassendere Auswirkungen auf deren Lebensumfeld haben. Denn die legitimen Bereiche staatlicher Beteiligungen vervielfältigen sich durch eine Expansion in nicht-arbeitsverwalterische Handlungsbereiche wie aufsuchende Dienste, Mitnahme zur Maßnahme, Begleitung zu Bera- 
tungsstellen und Ärzten, bis hin zur Recherche nach Unterlagen in den Privatwohnungen der Teilnehmenden.

Elemente dieses Vorgehens weisen eine lange Tradition auf: Schon seit dem 16. Jahrhundert fungiert die Regierung als Instrument, das über die richtige Lenkung der Individuen, Güter und Reichtümer ökonomische Prinzipien in die Gestaltung einführt (Foucault 2005a: 155f.). Die Steuerungsinstrumente des New Public Managements entwickeln diese Tradition weiter. Sie installieren nicht erst ökonomische Steuerungsformen und statistische Instrumente, sondern führen zu einer Effektivierung der Steuerungsinstrumente der Arbeitsverwaltung nach ökonomischen Prinzipien. Herausragendes Beispiel ist die Ablösung der Input- zu Gunsten einer Output-Steuerung: Die Output-Steuerung charakterisiert eine $E r-$ gebnisorientierung mittels Instrumenten der Kosten- und Leistungsrechnung, Leistungsmessungssysteme und die Definition von Produkten respektive OutputZielen (Nullmeier 2011). Im Bereich der Arbeitsverwaltung werden Ergebnisse der beschäftigungsfördernden Maßnahmen so insbesondere in quantifizierbaren Vermittlungsquoten von erwerbslosen Hilfebedürftigen in den ersten Arbeitsmarkt gemessen, die im Rahmen von Zielvereinbarungen vom Bundesministerium für Arbeit und Soziales bis hin zu den lokalen SGB-II-Trägern ausgehandelt und festgehalten werden. Die Institutionen der Arbeitsverwaltung passen sich zwar einerseits den Strukturen des New Public Managements an, doch gleichzeitig werden dessen Implikationen teilweise umgangen, indem beispielsweise Maßnahmen Sozialer Aktivierung implementiert werden, auch wenn diese im Controlling strukturell vergleichsweise weniger effektiv erscheinen. Die Jobcenterleitung beziehungsweise Maßnahmenplanung geht mit der Ermöglichung von Maßnahmen Sozialer Aktivierung häufig ein gewisses Risiko ein, denn Maßnahmenmittel für langzeitarbeitslose, schwer vermittelbare Personen bringen eine weniger hohe Vermittlungsquote in den ersten Arbeitsmarkt als Maßnahmen für eine arbeitsmarktnahe Klientel. Um die Aktivierung aller Personen im SGBII-Bezug zu erreichen, werden neue und in der Wahrnehmung der AkteurInnen passgenauere sozial aktivierende Instrumente geschaffen. Hier greifen nicht allein Effizienzgedanken, sondern auch der Beratungswunsch der Fachkräfte im Auftrag einer umfassenden Aktivierung und des Enablings der Personen sowie teilweise sozialpolitische Motive, um Vorbedingungen der Erwerbstätigkeit herzustellen. Zur Illustration die Reflektion einer Teamleiterin eines SGB-II-Trägers über die Erfolgsdefinition:

»Erfolg ist immer die Frage, wie man den misst. Also Erfolg ist entweder, wenn man ganz harte Zahlen sagt Integrationen, hat jemand das Arbeiten angefangen oder ist Erfolg auch etwas, das man sagt, es hat jemand, der sich jetzt die letzten Jahre überhaupt nicht mehr 
beworben hat, der hat sich jetzt wieder beworben - ist das auch ein Erfolg. [...] Oder ist jemand, der bisher jede Maßnahme verweigert hat und mit [...] einem Krankzettel kam, der jetzt plötzlich durchgehalten hat, ist das auch ein Erfolg? Also das ist für uns schon ein Erfolg, sonst würden wir solche Maßnahmen ja nicht kaufen.« (Teamleiterin, Interview04Regio02)

Der Durchführung von Maßnahmen Sozialer Aktivierung stehen, trotz der garantierten formalen Freiheit der lokalen Maßnahmengestaltung, Hürden im Wege. Denn durch die Zielsteuerung nach vordefinierten Vermittlungsquoten und Mittelkürzungen werden Gelder von den im Feld eindringlich geforderten Maßnahmen Sozialer Aktivierung abgezogen (etwa Interview07Regio02), um eine höhere beziehungsweise die geforderte Vermittlungsquote erzielen zu können. Denn mit der Neuorganisation der Maßnahmen an arbeitsmarktzentrierten Zielen (Dörre et al. 2013, Ludwig-Mayerhofer et al. 2009, Oschmiansky/Ebach 2009, Sell 2006) und einer seit 2011 erfolgten Mittelreduzierung für Eingliederungsleistungen, etwa im Bereich der Maßnahmen zur Aktivierung und beruflichen Eingliederung von über 1,6 Millionen im Jahr 2012 auf rund 1,2 Millionen Maßnahmenteilnahmen ab 2011, ${ }^{6}$ wird eine inhaltliche Engführung von personenbezogenen Arbeitsmarktdienstleistungen befördert (Kolbe 2012: 204). So kann eine personalisierte Vermittlung und Beratung mit kooperativem Handeln der Fachkräfte vor allem dann verwirklicht werden, wenn diese auch über Handlungsspielräume in der Maßnahmenbeschaffung verfügen und geweitete Erfolgsindikatoren in die Qualitätsmessung eingehen. So könnte in hier ansetzenden Studien untersucht werden, wie sich Erfolge in Maßnahmen Sozialer Aktivierung objektiv beurteilen lassen können. Die Arbeitsverwaltung befindet sich in einem Strukturdilemma, da sie sowohl arbeitsmarktpolitische als auch sozialintegrative Zielsetzungen einhalten muss (Harrach et al. 2000). Weiter müssen hier divergierende Gerechtigkeitsnormen in der Vermittlungspraxis ausbalanciert werden: einerseits die Gleichbehandlung Vieler über allgemeine Rechtsnormen, die auf Einzelne wirken und andererseits das Angebot von Arbeitsmarktdienstleistungen, die auf individuelle Bedürfnisse zugeschnitten sind (Steinke et al. 2012: 42f.). Auch die soziale Arbeit, die insbesondere in Maßnahmen Sozialer Aktivierung häufig ein elementarer Bestandteil ist, findet sich in einem solchen Dilemma, denn diese muss sowohl heterogene Bedürfnisse der Maßnahmenteilnehmenden als auch die Strukturen und Vorgaben des Aktivierungsdiskurses be-

6 Wie auch in der Tabelle 4: Teilnahmen in Maßnahmen zur Aktivierung und beruflichen Eingliederung ersichtlich (S. 125). 
dienen. Kratz sieht hier ein Konfliktfeld, das auf Basis der teilweise konträren Anweisungen und Logiken der Arbeitsvermittlung sowie der Sozialpolitik entsteht und die Gestaltung helfender Strukturen in der Interaktion beeinträchtigt (Kratz 2013b). Schönig führt aus, dass soziale Dienste im Kontext der Aktivierungspolitik verstärkt den Erfolg ihrer Arbeit nachweisen müssen und führt dies zurück auf die Verlagerung von monetären Sozialtransfers hin zu personenorientierten sozialen Diensten (Schönig 2006: 34). Dies findet seinen Ausdruck auch im Rahmen von Maßnahmen Sozialer Aktivierung, die unter dem Druck stehen, einen Wirkungsnachweis erbringen zu müssen. Die Wirkungsanalysen müssten jedoch auch weiche Faktoren wie sozialintegrative und persönlichkeitsstabilisierende Aspekte einbeziehen, um die Kosten-Nutzen-Analyse aussagekräftiger zu gestalten. Grundsätzlich sollte aber eine detaillierte zentrale Steuerung bis in die Richtwerte für die Abschlüsse von Eingliederungen sowie globale Indikatoren reflexiv eingesetzt werden, um nicht die Vermittlung allein auf leicht vermittelbare Personengruppen zu konzentrieren (Baethge-Kinsky et al. 2007: 62f., Bieback 2009: 205). »Zum anderen gibt es ein offensichtliches Spannungsverhältnis zwischen der Norm (»Mehr dezentrale Verantwortung für die Arbeitsagenturen«) und dem Steuerungszugriff durch die Regionaldirektionen bis hinein in die Prozessgestaltung in den Agenturen.« (Sell 2006: 145) So wurden Elemente der Konditionalsteuerung und der wirkungsorientierten (Ziel-)Steuerung in eine neue Form von Regelsteuerung überführt (Schütz 2012: 245). Denn obwohl die Ziele der wirkungsorientierten Steuerung priorisiert werden, erfolgt deren Umsetzung nach standardisierten Prozessregeln und auch die Umsetzungskontrolle enthält (neben In- und Outputs auf das Wirkungsergebnis) Kennzahlen für die Prozesskonformität. Letztlich führt dies Schütz zufolge dazu, dass für dezentrale Steuerungsimpulse und Handlungsspielräume weniger Möglichkeiten als im alten Bürokratiemodell liegen und zudem das Controlling expandiert (2012: 245f.).

Dennoch werden diese innovativen Maßnahmen eingesetzt, da es nach Aussagen der AkteurInnen für die Arbeit mit Langzeitarbeitslosen teilweise eines intensiveren Kontaktes zum Erreichen der Klientel bedarf als er über die SGB-IITräger-Beschäftigten bislang realisiert wurde. Dieser intensivere Kontakt konnte zunächst, so einige AkteurInnen, nur durch das legale Umgehen gesetzlicher Hürden aufgenommen werden, indem die Vernetzung beteiligter Institutionen gesteigert wurde und/oder indem weitere Institutionen hinzugezogen wurden. Denn Maßnahmen Sozialer Aktivierung beinhalten oder vermitteln zu Leistungen, die überwiegend durch andere Träger übernommen werden, also zum Beispiel aufsuchende Dienste (Leistung des Jugendamtes im U25-Bereich), Kinderbetreuung sichern (kommunale Leistung) oder therapeutische Anteile besitzen (Leistungen von Krankenkassen beziehungsweise Rentenversicherungsträgern). 
Erfolgreiche Kooperationen von verschiedenen Leistungsträgern, wie etwa dem Jobcenter, Krankenkassen und Beratungsstellen ${ }^{7}$ können dabei von einem aufwendigen und umfangreichen Entstehungs- sowie Aufrechterhaltungsprozess begleitet sein. Doch diese Leistungen fokussieren gerade die sehr arbeitsmarktferne Klientel, deren Problemlagen überwiegend an den Grenzen zwischen dem SGB II und anderen Leistungsträgern angesiedelt sind. Daher wird eine starke Vernetzung der jeweiligen Kostenträger vehement vertreten, was überdies der Befürchtung der Umlagefinanzierung von anderen Leistungsträgern entgegenwirken würde. Doch zum Teil erscheint in der Alltagspraxis die Umgehung der jeweiligen Hürden und die Gewährung von Leistungen anderer Leistungsträger effektiver, indem die Leistungen gebündelt und extern über einen Maßnahmenträger beschafft werden können. Beispielsweise gehören aufsuchende Dienste originär zu den Leistungen des Jugendamtes, doch bei einer Klientel, zu der der Zugang als erschwert eingeschätzt wird, können auch über sozial aktivierende Maßnahmen aufsuchende Dienste durch Maßnahmenträger eingekauft werden. Des Weiteren kann durch Mischfinanzierungen zum Teil eine Nachbetreuung sowie intensive Einzelarbeit mit den Teilnehmenden beim Maßnahmenträger in das Gesamtkonzept einfließen, die rechtlich nicht aus einer Hand leistbar wären.

Die Implementierung von Maßnahmen Sozialer Aktivierung zeugt so einerseits von der Lernfähigkeit der Institutionen der Arbeitsverwaltung, denn letztlich wurde ein neues Standardinstrument geschaffen und damit die bürokratischen Abläufe bedeutend vereinfacht. Andererseits zeigt es jedoch auch die Integration widerstreitender Positionen im Aktivierungsdiskurs, denn obwohl eine kurzfristige Erwerbsintegration anvisiert wird, entsteht ein langfristig integrierendes Standardinstrument. Wie Srubar dies für die Macht in der Politik ausmacht, werden in Machtdiskursen Gegensätze verklammert und damit eine höhere Effektivität sowie Immunität ermöglicht (Srubar 2006). Eine ähnliche Funktion schreiben auch Boltanski und Chiapello der Kritik im Kapitalismus und Foucault sozialen Bewegungen mit ihren Befreiungsversuchen und Freiheitsvisionen $\mathrm{zu}$ - letztlich wird der Kapitalismus anpassungsfähiger und immunisiert respektive effektivere Regierungsweisen etabliert (Boltanski/Chiapello 2006, Maurer 2006: 247). Dementsprechend wird das von der Erwerbsintegration zur Minderung sozialstaatlicher Passivleistungen, heruntergestufte Ziel der aktivierenden Arbeitsmarktpolitik bedient. Da unter anderem die zurückgegangene absolute

7 Vergleiche Kapitel 5.1.4 Fallbeispiel einer Maßnahme zur Bereitstellung psychischphysischer Unterstützungsangebote sowie gesundheitsorientierter Beschäftigungsvermittlung. 
Erwerbslosigkeit der vergangenen Jahre auf einen damit einhergehenden Mentalitätswechsel zurückgeführt wird, der eine Steigerung der Erwerbsorientierung von arbeitslosen Transferleistungsbeziehenden bewirkt habe (Dörre et al. 2013). Doch eine konstant hohe Erwerbsorientierung von erwerbslosen SGB-II-Leistungsbeziehenden ist vielfach nachgewiesen worden (Dörre et al. 2013, Sondermann 2013, Ludwig-Mayerhofer et al. 2009). Resümierend lässt sich sagen, dass in der vorliegenden Studie der Aktivierungsdiskurs und sein Praxisfeld insbesondere im Hinblick auf den Bereich der Beschäftigungsförderung und deren Reaktion auf eine sehr arbeitsmarktferne Klientel betrachtet wurden. Dieser Ausschnitt des Diskurses ist in einen weitreichenderen politischen Diskurs eingebunden, der teilweise analytisch und durch die Heranziehung weiterer theoretischer Arbeiten berücksichtigt wurde. Doch:

»Jedes Kraftverhältnis impliziert zu jedem Zeitpunkt eine Machtbeziehung (die gewissermaßen dessen momentaner Querschnitt ist), jede Machtbeziehung verweist, insofern das ihre Wirkung, aber auch insofern das ihre Bedingung der Möglichkeit ist, auf ein politisches Feld, dem sie angehört.« (Foucault 2005b: 132)

So könnte hier ein intensiverer Blick auf das weitergehende Diskursfeld des politisch-rechtlichen Aktivierungsdiskurses weitere innerdiskursive Positionen zur (sozialen) Aktivierung sowie Machtbeziehungen im Diskurs erörtern. Eine derart konzipierte Diskursanalyse könnte nun klären, ob die Hybridisierungstendenz der erweiterten Aktivierung dazu führt, dass der Aktivierungsdiskurs an seinen Enden >ausfranst` oder negiert wird. Verallgemeinernd wird im Staatskonzept Steuerung zu Gunsten eines flexiblen Staates en passant invertiert. Schon Foucault antwortet auf die Frage von R. Lefort, ob der Vorteil, den Macht aus der Verschleierung der aus dem Sicherheitssystem erwachsenden Zwänge zieht, letztlich in ihrer Perpetuierung liegt, da das System durch die so gewonnene Flexibilität Schläge besser verarbeiten und leichter parieren kann:

»Die Entwicklung der Staaten geht heute nicht mehr in Richtung größerer Rigidität, sondern in Richtung größerer Geschmeidigkeit und Flexibilität. Der Staat hat die Möglichkeit vorzurücken und zurückzuweichen. Die staatlichen Strukturen sind so flexibel, dass ihr Verhalten an manchen Punkten sogar wie ein Zurückweichen des Staatsapparats erscheinen kann: die Atomisierung der Produktionseinheiten, eine größere regionale Autonomie das sind Dinge, die der Entwicklung des Staates scheinbar zuwider laufen.« (Lefort/Foucault 2005: 143) 
In dem Phänomen der Maßnahmen Sozialer Aktivierung zeigt sich zudem, dass die Zielsetzung einer langfristigen Erwerbsintegration um die soziale Aktivierung erweitert wird, indem persönlichkeitsfördernde und alltagsstabilisierende Aspekte in die Maßnahmengestaltung einfließen. Somit wird eine soziale Integration ermöglicht, die negative Zuschreibungen auf leistungsbeziehende Erwerbslose durch eine Gegenleistung in Form der Maßnahmenteilnahme nivelliert. Daneben ist in den einzelnen Maßnahmen meist eine Integration in die Gruppen- und Sozialstrukturen intendiert. Somit werden die Maßstäbe expandiert, unter denen der legitime Leistungsbezug im Sinne des Diskurses gemessen wird, jedoch auch die Selbsttechnologien der Subjekte erweitert, dank der sie im Foucaultschen Sinne selbst zu einer glücklichen Denk- und Lebensweise gelangen (Foucault 2005c: 968), indem sie sich in der Maßnahmenteilnahme trotz SGB-II-Leistungsbezug als legitim handelnd betrachten können.

»Und für den flexibel-kapitalistischen Sozialstaat ist diese ökonomische Sozialisation des Aktivbürgers zugleich ein Akt seiner politischen Re-Sozialisierung, denn die aktive Ausübung von selbststeuerndem und eigenverantwortlichem Handeln auf Märkten ist zugleich immer auch - so der sozialpolitische Lehr- und Lernauftrag - eine soziale Tat im Sinne der »mikropolitischen« Verantwortungsübernahme für das Gemeinwohl.« (Lessenich 2012b: 61)

In diesem Zusammenhang werden dauerhaft, öffentlich geförderte Beschäftigungsverhältnisse diskutiert, als Integrations- und Teilhabeoptionen für eine spezifische Klientel. Dagegen gelten solche Elemente dem Aktivierungsdiskurs als unangemessen. Dennoch stellt sich die Frage, welche Teilhabechancen über Erwerb ermöglicht werden sollen, wie dies beispielsweise in den Äußerungen der Teamleiterin eines SGB-II-Trägers anklingt:

»einfach noch mal neu über >Arbeit` reden. Über Definitionen von Arbeit. Ist eigentlich sozialversicherungspflichtige Beschäftigung das Nonplusultra? Obwohl wir das immer mehr kaputt machen als Struktur? Mit diesen 400€-Jobs haben wir ganze Berufsbilder tot gemacht. Ja. Friseur, Handel, Reinigung, Gastronomie. Ist ja alles nur noch 400€-Jobs, kann keiner von leben, geschweige denn in die Sozialversicherung einzahlen. [...] Und Ehrenamt ist ja nicht mehr Ehrenamt, wenn jemand bei uns Leistungen bezieht. Dann kriegt er nämlich im Monat 600, 700 oder 800 Euro dafür.« (Teamleiterin, Interview02Regio05)

So ist der Anteil atypischer Beschäftigungsverhältnisse in den zwei Jahrzehnten, von 1992 bis 2012, von 13 auf 22 Prozent gestiegen (Statistisches Bundesamt 
2013: 339f.). Weiter sind trotz gesunkener Anzahl von Menschen in der Grundsicherung im Jahr 2012 unter zwei Millionen (BA 2013e: 6) gleichzeitig nur zwei von fünf Arbeitslosengeld II-Beziehenden arbeitslos (BA 2013a: 10), die anderen gehen einer Erwerbstätigkeit nach, deren Entlohnung das Existenzminimum nicht überschreitet. Sowohl der vermehrte Zugang in Beschäftigung, als auch die Veränderungen in den Beschäftigungsformen und die zunehmende Lohnungleichheit können partiell auf die bisherige aktivierende Arbeitsmarktpolitik zurückgeführt werden (Klinger et al. 2013, Rhein 2013, Dörre et al. 2013). Auch europaweit ist die Zahl atypischer Beschäftigungsformen angestiegen (G. Schmid 2012). Dies kann einerseits als Förderung der Integration von Erwerbslosen gesehen werden, denn die atypischen Beschäftigungsformen bieten Einstiegsmöglichkeiten in den Arbeitsmarkt, auch wenn diese nicht immer existenzsichernd sind (Bruckmeier et al. 2013). Gleichzeitig wird deutlich, dass der Aufstocker-Status insgesamt stabil ist, denn gut 60 Prozent der Aufstocker von 2010 waren bereits 2009 erwerbsfähige Leistungsbeziehende (Bruckmeier et al. 2013: 7). ${ }^{8}$ Aufstocken und atypische Arbeitsverhältnisse könnten jedoch auch als prekäre Form der Integration gesehen werden, wenn der Prekarisierungsthese von Dörre et al. gefolgt wird:

»Das aktivierende Arbeitsmarktregime ist erfolgreich, weil es die Langzeitarbeitslosigkeit reduziert, indem es atypische und prekäre Beschäftigung fördert. Diese Problemverschiebung ermöglicht den Übergang von einer fordistischen Vollbeschäftigungs- zu einer prekären Vollerwerbsgesellschaft.« (Dörre et al. 2013: 33)

Denn in einer prekären Vollerwerbsgesellschaft sind erwerbstätige Personen, die dennoch Sozialleistungen beziehen und erwerbsfähig sind, nach den Annahmen der vorliegenden Studie von negativen Zuschreibungen betroffen, da sie weiterhin Sozialleistungen erhalten - auch wenn sie einen Teil des Lebensunterhaltes

8 Als Faktoren, die die Transformation einer Tätigkeit in ein »Aufstocker-Arbeitsverhältnis« definieren oder begünstigen, gelten hier: geringe Qualifikation, niedriger Stundenlohn, schwere gesundheitliche Einschränkungen, höheres Lebensalter, Minijob, befristete Tätigkeiten oder Zeitarbeit, ein zusätzliches Kind im Haushalt, der Status Alleinerziehend und eine hohe regionale Arbeitslosigkeit (Bruckmeier et al. 2013: 8). Ein höherwertiger Berufsabschluss, weniger Kinder, ein Zuwachs an Erwerbstätigen in der Familie und - naheliegender Weise - ein höherer Stundenlohn erhöhen dagegen die Chance, in Erwerbstätigkeit nicht mehr von der Grundsicherung abhängig zu sein (Bruckmeier et al. 2013). 
selbst erarbeiten. Denn wenn Arbeitsmarktrisiken individualisiert werden, besteht die Gefahr, strukturelle Bedingungen von Arbeitslosigkeit nicht mehr wahrzunehmen. So erkennen beispielsweise Schütz und KollegInnen bei Vermittlungsfachkräften eine Verharmlosung oder Übertragung von Arbeitsmarktproblemen auf Kundinnen und Kunden (Schütz et al. 2011: 263). Damit werden auch Grenzen der aktivierenden Arbeitsmarktpolitik deutlich. Sind die Aufnahmekapazitäten des regionalen oder sektoralen Arbeitsmarktes erschöpft, können auch intensive Förderung und Sanktionierung durch die Fachkräfte wenig zu einer Arbeitsmarktintgration beitragen, genauso wie bei Personen, die zwar den Status der Erwerbsfähigkeit tragen, jedoch unter massiven, dauerhaften gesundheitlichen Einschränkungen leiden (Kaltenborn/Kaps 2013, Schubert et al. 2013, Schütz et al. 2011, Oschmiansky 2010, Henkel et al. 2009, Ames 2008). Daneben kann sich die kommunikative Kooperation zwischen Fachkräften und erwerbslosen Leistungsbeziehenden zum Selbstzweck entwickeln, wenn keine realistische Chance zur Erwerbsintegration besteht und damit beide Parteien belasten (Ludwig-Mayerhofer et al. 2009: 219ff.). Zudem verweisen der wachsende Leistungsbezug trotz Erwerbstätigkeit, die atypische Beschäftigung und die Maßnahmen Sozialer Aktivierung auf die Frage, wie langfristig mit Personen umgegangen werden soll, die dauerhaft nicht in Vollzeitbeschäftigungen integrierbar sind? Diese Frage stellt sich beispielsweise bei signifikanten gesundheitlichen oder sozialen Einschränkungen. Unter Umständen ist es angebracht, die Kritierien von Erwerb neu zu denken, unter Berücksichtigung seiner sozialen Funktion der Inklusion in die Arbeitsgesellschaft und gegebenenfalls die beschäftigungsfördernden Instrumente anzupassen. Denn letztlich bedeutet $>$ Regierungく, den Komplex von Menschen und Dingen zu lenken und gemäß der Foucaultschen Schiffsmetapher die vielseitigen Ansprüche in der Lenkungsaufgabe zu berücksichtigen:

»es bedeutet, einen Zusammenhang herzustellen zwischen den Seeleuten, die man am Leben erhalten, dem Schiff, das man bewahren und der Ladung, die man in den Hafen bringen muss, und deren Beziehungen wiederum zu all jenen Ereignissen wie den Winden, den Klippen und den Unwettern.« (Foucault 2000: 51). 



\section{Quellen- und Literaturverzeichnis}

Abelshauser, W. (2009a): »Strukturelle Arbeitslosigkeit: Eine Diagnose aus historischer Perspektive«, in: Jahrbuch für Wirtschaftsgeschichte, Jg. 2, Heft 50, S. 49-262.

Abelshauser, W. (2009b): »Wandlungen der Sozialen Marktwirtschaft. Des Kaisers neue Kleider?« (= RHI-Positionen 7/2009), München.

Achatz, J./Bruckmeier, K./Buch, T./Burkert, C./Dietrich, H./Dietz, M./Garloff, A./Gundert, S./Hell, S./Hohendanner, C./Jahn, K./Kleinert, C./Koller, L./Lehmer, F./Matthes, B./Niebuhr, A./Schells, B./Trappmann, M./Walwei, U./Wapler, R./Ziegler, K. (2013): »Übergänge am Arbeitsmarkt und Qualität von Beschäftigung«, in: H. Brücker/S. Klinger/J. Möller (Hg.), Handbuch Arbeitsmarkt 2013. Analysen, Daten, Fakten, Bielefeld: Bertelsmann, S. 141-203.

Achatz, J./Fehr, S./Schels, B./Wolff, J. (2012): Ein-Euro-Jobs, betriebliche und schulische Trainingsmaßnahmen: Wovon junge Arbeitslose im SGB II am meisten profitieren (= IAB-Kurzbericht 6/2012), Nürnberg, S. 1-8.

Allmendinger, J./Giesecke, J./Hipp, L. (2012): Mehr Jobs oder nur mehr schlechte Jobs? Die Entwicklung atypischer Beschäftigung in Europa (=WZBrief Arbeit 13/2012), Berlin.

Ames, A. (2008): Arbeitssituation und Rollenverständnis der persönlichen Ansprechpartner/-innen nach $\S 14$ SGB II. Eine Studie im Auftrag der HansBöckler-Stiftung, Düsseldorf.

Angermuller, J./Nonhoff, M./Herschinger, E./Maggilchrist, F./Reisigl, M./Wedl, J./Wrana, D./Ziem, A. (Hg.) (2014): Diskursforschung. Ein interdisziplinäres Handbuch, Band 1: Theorien, Methodologien und Kontroversen, Bielefeld: transcript.

Angermüller, J. (2005): Sozialwissenschaftliche Diskursanalyse in Deutschland: zwischen Rekonstruktion und Dekonstruktion, in: R. Keller/A. Hirseland/W. 
Schneider/W. Viehöver (Hg.), Die diskursive Konstruktion von Wirklichkeit, Konstanz: UVK, S. 23-48.

Angermüller, J./van Dyk, S. (2010): Diskursanalyse meets Gouvernementalitätsforschung. Perspektiven auf das Verhältnis von Subjekt, Sprache, Macht und Wissen, Frankfurt/New York: Campus.

Apel, H./Engels, D. (2013): Zentrale Ergebnisse der unabhängigen wissenschaftlichen Untersuchung zur Erforschung der Ursachen und Auswirkungen von Sanktionen nach $\S 31$ SGB II und nach dem SGB III in NRW im Auftrag des Ministeriums für Arbeit, Integration und Soziales des Landes Nordrhein-Westfalen, Köln.

Aram, E./Sommer, J./Timptner, S. (2012): Abschlussbericht Evaluation des Bundesprogramms »STÄRKEN vor Ort«, herausgegeben vom Bundesministerium für Familie, Senioren, Frauen und Jugend, Berlin.

Artus, I. (2014): Mitbestimmung und Leiharbeit, in: WSI-Mitteilungen, Jg. 2, S. 113-121.

Artus, I. (2013): Ausländische Systeme der industriellen Beziehungen, in: H. Hirsch-Kreinsen/H. Minssen (Hg.), Lexikon der Arbeits- und Industriesoziologie, Berlin: Edition Sigma, S. 106-111.

Artus, I. (2011): Gewerkschaftliche Interessenvertretung im Niedriglohnsektor und der Streik der französischen Travailleurs sans papiers, in: T. Haipeter/K. Dörre (Hg.), Gewerkschaftliche Modernisierung, Wiesbaden: VS, S. 208230.

BA - Bundesagentur für Arbeit (2014): Der Arbeitsmarkt im Jahr 2013: Stabiler Arbeitsmarkt trotz schwachem Wirtschaftswachstum, Presse Info 002 vom 07.01.2014, Nürnberg, abgerufen am 07.01.2014 von http://www.arbeits agentur.de/web/content/DE/Presse/Presseinformationen/ArbeitsundAus bildungsmarkt/Detail/index.htm?dfContentId=L6019022DSTBAI620379.

BA - Bundesagentur für Arbeit (2016): Zugang von Teilnehmern in Maßnahmen zur Aktivierung und beruflichen Eingliederung, Statistische Auswertung, Nürnberg.

BA - Bundesagentur für Arbeit (2013a): Akkreditierung und Zulassung ab 01.04.2012, abgerufen am 07.01.2014 von http://www.arbeitsagentur.de/ web/content/DE/Institutionen/Traeger/AkkreditierungundZulassung/Detail/in dex.htm?dfContentId=L6019022DSTBAI524613.

BA - Bundesagentur für Arbeit (2013b): Arbeitslosengeld II/Sozialgeld, Nürnberg.

BA - Bundesagentur für Arbeit (2013c): Arbeitsmarktprogramme der Länder. Anlage zur Beratungsunterlage Nr. 49/2013, Nürnberg. 
BA - Bundesagentur für Arbeit (2013d): Erwerbstätige Arbeitslosengeld II-Bezieher, Statistik zum Arbeitsmarkt in Zahlen, Nürnberg, abgerufen am 01.12.2013 von http://statistik.arbeitsagentur.de/nn_31986/SiteGlobals/ Forms/Rubrikensuche/Rubrikensuche_Form.html?view=processForm\&resou rceId $=210368 \&$ input_ $=\&$ pageLocale $=$ de\&topicId $=17710 \&$ year_month $=201$ $310 \& y e a r \_m o n t h . G R O U P=1 \&$ search $=$ Suchen .

BA - Bundesagentur für Arbeit (2013e): Jahresbericht 2012 SGB II/Grundsicherung. Zahlen. Daten. Fakten, Nürnberg.

BA - Bundesagentur für Arbeit (2013f): Organisation, abgerufen am 03.01.2014 von http://www.arbeitsagentur.de/nn_27200/Navigation/zentral/Servicebe reich/Ueber-Uns/Aufbau-und-Organisation/Aufbau-und-Organisation-Nav. html.

BA - Bundesagentur für Arbeit (2012a): Selbstverwaltung, abgerufen am 03.01. 2014 von http://www.arbeitsagentur.de/nn_27196/Navigation/zentral/Ser vicebereich/Ueber-Uns/Aufbau-und-Organisation/Selbstverwaltung/Selbst verwaltung-Nav.html.

BA - Bundesagentur für Arbeit (2012b): SGB II Fachliche Hinweise. Freie Förderung SGB II nach $\S 16$ f SGB II, Nürnberg, abgerufen am 04.11.2013 von http://www.arbeitsagentur.de/zentraler-Content/HEGA-Internet/A05-BeruflQualifizierung/Dokument/HEGA-03-2012-Freie-Foerderung-SGB-II-Anla ge.pdf.

BA - Bundesagentur für Arbeit (2011): SGB II - Arbeitshilfe Arbeitsgelegenheiten $(\mathrm{AGH})$ nach $\S 16 \mathrm{~d}$ SGB II, Nürnberg, abgerufen am 01.12.2013 von http://www.arbeitsagentur.de/zentraler-Content/HEGA-Internet/A06-Schaf fung/Publikation/GA-SGB-2-NR-21-2009-07-14-Anlage.pdf.

BA - Bundesagentur für Arbeit (2010a): Aufgaben im Beschaffungsprozess, Informationen aus dem Intranet, erhalten am 15.11.2011.

BA - Bundesagentur für Arbeit. (2010b): Der Einkauf von Arbeitsmarktdienstleistungen (AMDL), Informationen aus dem Intranet, erhalten am 15.11. 2011.

BA - Bundesagentur für Arbeit (2008a): Grundsicherung für Arbeitsuchende. Zahlen. Daten. Fakten. Jahresbericht 2007, Nürnberg.

BA - Bundesagentur für Arbeit (2008b): SGB II. Grundsicherung für Arbeitsuchende. Arbeitslosengeld II/Sozialgeld, Nürnberg.

BA - Bundesagentur für Arbeit (2006): SGB II. Grundsicherung für Arbeitsuchende. Zahlen. Daten. Fakten. Jahresbericht 2005, Nürnberg.

BA - Bundesagentur für Arbeit Regionaldirektion Regionales Einkaufszentrum Regio02 (2010): Vergabeunterlagen zur Öffentlichen Ausschreibung eines Rahmenvertrages über Aktivcenter nach $\S 16$ Abs. 1 Zweites Buch Sozial- 
gesetzbuch (SGB II) i.V.m. § 46 Abs. 1 Satz1 Drittes Buch Sozialgesetzbuch (SGB III)/2010, Regio02.

BA - Bundesagentur für Arbeit Regionales Einkaufszentrum Regio02 (2010): Einkauf Arbeitsmarktdienstleistungen, Information aus dem Intranet des REZ Regio02, erhalten am 08.11.2010.

Baethge-Kinsky, V./Bartelheimer, P./Henke, J./Wolf, A./Land, R./Willisch, A./Kupka, P. (2007): Neue soziale Dienstleistungen nach SGB II (= IABForschungsbericht 15/2007), Nürnberg, S.1-83.

Beck-Gernsheim, E. (1986): „Von der Liebe zur Beziehung? Veränderungen im Verhältnis von Mann und Frau in der individualisierten Gesellschaft«, in: Soziale Welt, Sonderband 4: Die Moderne - Kontinuitäten und Zäsuren, S. 209-233.

Behrend, O. (2007): " $>\ldots$ das geht zu Lasten eigener Emotionalität - Instrumente zur Kundensteuerung in Arbeitsverwaltungen aus Sicht von Arbeitsvermittlern«, in: W. Ludwig-Mayerhofer/ O. Behrend/A. Sondermann (Hg.), Fallverstehen und Deutungsmacht. Akteure in der Sozialverwaltung und ihre Klienten, Opladen: Barbara Budrich, S. 97-117.

Berger, L. P./Luckmann, T. (2004): Die gesellschaftliche Konstruktion der Wirklichkeit. Eine Theorie der Wissenssoziologie, Frankfurt am Main: Fischer.

Bescherer, P./Röbenack, S./Schierhorn, K. (2008): »Nach Hartz IV: Erwerbsorientierung von Arbeitslosen«, in: Aus Politik und Zeitgeschichte, Jg. 58, Nr. 33-34, S. 19-24.

Bieback, K.-J. (2009): »Kooperation im Zwangsverhältnis. Teilhaberechte und Vertragsstrukturen in der Arbeitsmarktverwaltung «, in: Zeitschrift für Rechtssoziologie, Jg. 30, Heft 2, S. 185-213.

Blien, U./Hirschenauer, F./Kaufmann, K./Moritz, M./Vosseler, A. (2011): Leistungsmessung von SGB-II-Trägern: Die Bildung von Vergleichstypen trägt regionalen Unterschieden Rechnung (= IAB-Forum 2/2011), Nürnberg, S. 82-89.

Blumer, H. (1954): „What is Wrong with Social Theory?«, in: American Sociological Review, Jg. 19, Heft 1, S. 3-10.

Blumer, H. (1940): »The Problem of the Concept in Social Psychology«, in: American Journal of Sociology, Jg. 45, S. 707-719.

BMAS - Bundesministerium für Arbeit und Soziales (2013a): Grundsicherung für Arbeitsuchende. Sozialgesetzbuch (SGB II). Fragen und Antworten, Bonn: BMAS.

BMAS - Bundesministerium für Arbeit und Soziales (2013b): Lebenslagen in Deutschland. Armuts- und Reichtumsberichterstattung der Bundesregierung. 
Forschungsprojekt Soziale Mobilität, Ursachen für Auf- und Abstiege. Studie für den 4. Armuts- und Reichtumsbericht der Bundesregierung im Auftrag des Bundesministeriums für Arbeit und Soziales, Bonn: BMAS.

BMAS - Bundesministerium für Arbeit und Soziales (2011a): A-Z der Arbeitsförderung. Nachschlagewerk zum Sozialgesetzbuch Drittes Buch (SGB III), Bonn: BMAS.

BMAS - Bundesministerium für Arbeit und Soziales (2011b): Factsheet zu Perspektive 50plus, abgerufen am 12.11.2013 von http://www.perspektive50 plus.de/fileadmin/user_upload/presse/Basispressemappe/P50plus_Factsheet_ 2011_final.pdf.

BMAS - Bundesministerium für Arbeit und Soziales (2010): Perspektive 50plus - Das Bundesprogramm, abgerufen am 13.09.2010 von http://www.perspekti ve50plus.de/perspektive_50plus/.

BMAS - Bundesministerium für Arbeit und Soziales (2008): Endbericht zur Evaluation der Experimentierklausel nach § 6c SGB II. Vergleichende Evaluation des arbeitsmarktpolitischen Erfolgs der Modelle der Aufgabenwahrnehmung »zugelassener kommunaler Träger« und »Arbeitsgemeinschaft«, Berlin.

BMWA - ehemaliges Bundesministerium für Wirtschaft und Arbeit (2005a): Hartz IV. Menschen in Arbeit bringen, Berlin.

BMWA - ehemaliges Bundesministerium für Wirtschaft und Arbeit (2005b): Vorrang für die Anständigen - Gegen Missbrauch, »Abzocke« und Selbstbedienung im Sozialstaat. Ein Report vom Arbeitsmarkt im Sommer 2005, Berlin, abgerufen am 22.07.2008 von http://www.sozialpolitik-aktuell. de/tl_files/sozialpolitik-aktuell/_Kontrovers/HartzIV/SGBIIMissbrauchskam pagne.pdf.

Bode, I. (2013): Die Infrastruktur des postindustriellen Wohlfahrtsstaats, Wiesbaden: VS.

Bode, I. (2011): »Soziale Dienstleistungen am Arbeitsmarkt«, in: A. Evers/R. G. Heinze/T. Olk (Hg.), Handbuch Soziale Dienste, Wiesbaden: VS, S. $317-$ 332.

Bode, I. (2005): Die Dynamik organisierter Beschäftigungsförderung: eine qualitative Evaluation, Wiesbaden: VS.

Bogner, A./Menz, W. (2009): Experteninterviews in der qualitativen Sozialforschung, in: A. Bogner/B. Littig/W. Menz (Hg.), Experteninterviews - Theorien, Methoden, Anwendungsfelder (3. überarbeitete Auflage), Wiesbaden: VS. 61-98.

Bogner, A./Littig, B./Menz, W. (2009): Experteninterviews - Theorien, Methoden, Anwendungsfelder (3. überarbeitete Auflage), Wiesbaden: VS. 
Boltanski, L./Chiapello, È. (2006): Der neue Geist des Kapitalismus, Konstanz: UVK.

Boockmann, B./Koch, S./Rosemann, M./Stops, M./Verbeek, H. (2010): Aktive Arbeitsmarktpolitik. Fördern und Fordern aus Sicht der Vermittler (= IABKurzbericht 25/2010), Nürnberg, S. 1-8.

Bourdieu, P. (2000): Die zwei Gesichter der Arbeit. Interdependenzen von Zeitund Wirtschaftsstrukturen am Beispiel einer Ethnologie der algerischen Übergangsgesellschaft, Konstanz: UVK.

Bourdieu, P. (1998): »Prekarität ist überall«, in: P. Bourdieu: Gegenfeuer. Wortmeldung im Dienste des Widerstands gegen die neoliberale Invasion, Konstanz: UVK, S. 96-102.

BRH - Bundesrechnungshof (2006): Bericht an den Haushaltsausschuss und an den Ausschuss für Arbeit und Soziales des Deutschen Bundestages nach $\S 88$ Abs. 2 BHO. Durchführung der Grundsicherung für Arbeitsuchende. Wesentliche Ergebnisse der Prüfungen im Rechtskreis des Zweiten Buches Sozialgesetzbuches vom 19.05.2006, Bonn.

Brinkmann, U./Dörre, K./Röbenack, S./Kraemer, K./Speidel, F. (2006): Prekäre Arbeit. Ursachen, Ausmaß, soziale Folgen und subjektive Verarbeitungsformen unsicherer Beschäftigungsverhältnisse (= Friedrich-Ebert-Stiftung, Gesprächskreis Migration und Integration), Bonn.

Bröckling, U. (2012): »Der Ruf des Polizisten. Die Regierung des Selbst und ihre Widerstände«, in: R. Keller/W. Schneider/W. Viehöver (Hg.), Diskurs Macht - Subjekt. Theorie und Empirie von Subjektivierung in der Diskursforschung, Wiesbaden: VS, S. 131-144.

Bröckling, U. (2007): Das unternehmerische Selbst. Soziologie einer Subjektivierungsform, Frankfurt am Main: Suhrkamp.

Bröckling, U. (2000): »Totale Mobilmachung. Menschenführung im Qualitätsund Selbstmanagement«, in: U. Bröckling/S. Krasmann/T. Lemke (Hg.), Gouvernementalität der Gegenwart. Studien zur Ökonomisierung des Sozialen, Frankfurt am Main: Suhrkamp, S. 131-167.

Bröckling, U./Krasmann, S./Lemke, T. (2000): Gouvernementalität der Gegenwart. Studien zur Ökonomisierung des Sozialen. Frankfurt am Main: Suhrkamp.

Bruckmeier, K./Eggs, J./Himsel, C./Trappmann, M./Walwei, U. (2013): Aufstocker im SGB II. Steinig und lang - der Weg aus dem Leistungsbezug (= IAB-Kurzbericht 14/2013), Nürnberg, S. 1-8.

Brüsemeister, T. (2000): Qualitative Forschung - Ein Überblick (= Hagener Studientexte zur Soziologie Bd. 6), Wiesbaden: Westdeutscher Verlag. 
Budäus, D. (1994): Public Management: Konzepte und Verfahren zur Modernisierung öffentlicher Verwaltungen, Berlin: Ed. Sigma.

Bude, H. (2006): »Zumutungen und Anrechte. Zur Aktualität einiger Motive des Subsidiaritätsgedankens«, in: Mittelweg 36, Jg. 15, Heft 5, S. 26-40.

Butler, J. (2001): Psyche der Macht. Das Subjekt der Unterwerfung, Frankfurt am Main: Suhrkamp.

Butterwegge, C. (2006): Krise und Zukunft des Sozialstaates (3. erweiterte Auflage), Wiesbaden: VS.

BVerfG, Bundesverfassungsgericht (2007): Leitsatz zum Urteil des Zweiten Senats, 2 BvR 2433/04 vom 20.12.2007, Absatz-Nr. (1-228), Berlin.

Castel, R (2011): Die Krise der Arbeit. Neue Unsicherheiten und die Zukunft des Individuums, Hamburg: Hamburger Edition HIS.

Castel, R. (2000): Die Metamorphosen der sozialen Frage. Eine Chronik der Lohnarbeit, Konstanz: UVK.

Castel, R./Dörre, K. (2009): Prekarität, Abstieg, Ausgrenzung. Die soziale Frage am Beginn des 21. Jahrhunderts, Frankfurt am Main/New York: Campus.

CDU/CSU-Landesleitung/SPD (2013): Deutschlands Zukunft gestalten. Koalitionsvertrag zwischen CDU, CSU und SPD, Berlin.

Dann, S./Hamacher, C./Kirchmann, A./Klee, G./Kleimann, R./Rosemann, M./Strotmann, H. (2007): Evaluation der Experimentierklausel nach §6c SGB II - Vergleichende Evaluation des arbeitsmarktpolitischen Erfolgs der Modelle Aufgabenwahrnehmung >zugelassene kommunale Träger` und >Arbeitsgemeinschaft $<$ : Untersuchungsfeld I: >Deskriptive Analyse und Matching

Dengler, K. (2013): Effectiveness of Sequences of One-Euro-Jobs: Is it better to do more One-Euro-Jobs or to wait? (= IAB-Discussion Paper 16/2013), S. 179.

Dettmer, M./Gathmann, F./Wittrock, P. (2014): »SPD-Kernanliegen Mindestlohn: Nahles boxt ihr Prestigeprojekt«, in: Spiegel Online, abgerufen am 19.03.2014 von http://www.spiegel.de/politik/deutschland/mindestlohn-spdnahles-legt-gesetz-vor-widerstand-in-der-cdu-a-959653.html

Deutscher Bundestag (2013a): Das Dritte Buch Sozialgesetzbuch - Arbeitsförderung - (Artikel 1 des Gesetzes vom 24.03.1997, BGB1. I S. 594, 595), das zuletzt durch Artikel 11 des Gesetzes vom 19.10.2013 (BGB1. I S. 3836) geändert worden ist.

Deutscher Bundestag (2013b): Das Zweite Buch Sozialgesetzbuch - Grundsicherung für Arbeitsuchende - in der Fassung der Bekanntmachung vom 13.05.2011 (BGB1. I S. 850, 2094), das zuletzt durch Artikel 1 des Gesetzes vom 07.05.2013 (BGBl. I S. 1167) geändert worden ist. 
Deutscher Bundestag (2013c): Öffentliche Stellungnahme zur öffentlichen Anhörung von Sachverständigen am 12.04.2013, Ausschussdrucksache 17(11)1112, Berlin.

Deutscher Bundestag (2008a): Bericht zur Evaluation der Experimentierklausel nach $\S 6 \mathrm{c}$ des Zweiten Buches Sozialgesetzbuch, Bundestagsdrucksache 16/11488 vom 18.12.2008, Berlin.

Deutscher Bundestag (2008b): Gesetzentwurf der Bundesregierung. Entwurf eines Gesetzes zur Neuausrichtung der arbeitsmarktpolitischen Instrumente, Drucksache 16/10810 vom 08.11.2008, Berlin.

Deutscher Bundestag. (2003a): Drittes Gesetz für moderne Dienstleistungen am Arbeitsmarkt. Bundesgesetzblatt, Teil I Nr. 65 vom 23.12.2003, Bonn.

Deutscher Bundestag (2003b): Viertes Gesetz für moderne Dienstleistungen am Arbeitsmarkt vom 24.12.2003, Bonn.

Deutscher Bundestag (2002a): Erstes Gesetz für moderne Dienstleistungen am Arbeitsmarkt verkündet im Bundesgesetzblatt Teil I, Nr. 87 vom 30.12.2002, Berlin.

Deutscher Bundestag. (2002b): Zweites Gesetz für moderne Dienstleistungen am Arbeitsmarkt verkündet im Bundesgesetzblatt Teil I, Nr. 87 vom 23.12. 2002, Bonn.

Deutscher Bundestag (2001): Job-AQTIV-Gesetz. Gesetz zur Reform der arbeitsmarktpolitischen Instrumente vom 10.12.2001 verkündet im Bundesgesetzblatt Teil I, Nr. 66 vom 14.12.2001.

Dingeldey, I. (2010): »Agenda 2010: Dualisierung der Arbeitsmarktpolitik«, Aus Politik und Zeitgeschichte, Jg. 48, S. 17-24.

Dingeldey, I. (2007): »Wohlfahrtsstaatlicher Wandel zwischen >Arbeitszwang und >Befähigung««, in: Berliner Journal für Soziologie, Jg. 17, Heft 2, S. $189-209$.

Doerr, A./Kruppe, T. (2012): Bildungsgutscheine und Zertifizierung aus Sicht der Arbeitsverwaltung. Umfrageergebnisse aus der Bundesagentur für Arbeit (= IAB-Forschungsbericht 6/2010), Nürnberg, S. 1-46.

Dörre, K./Scherschel, K./Booth, M./Haubner, T./Marquardsen, K./Schierhorn, K. (2013): Bewährungsproben für die Unterschicht? Soziale Folgen aktivierender Arbeitsmarktpolitik, Frankfurt am Main: Campus.

Dollinger, B. (2006): »Zur Einleitung: Perspektiven aktivierender Sozialpädagogik «, in: B. Dollinger/J. Raithel (Hg.), Aktivierende Sozialpädagogik. Ein kritisches Glossar, Wiesbaden: VS, S. 7-22.

Dyk, S. van/Lessenich, S./Denninger, T./Richter, A. (2010): »Die >Aufwertung des Alters. Eine gesellschaftliche Farce«, in: Mittelweg 36, Jg. 19, Heft 5, S. 15-33. 
Egeler, R. (2012): Pressekonferenz >Niedriglohn und Beschäftigung 2010 «am 10.09.2012 in Berlin. Statement von Präsident Roderich Egeler, Berlin.

Eichler, L. (2013): System und Selbst. Arbeit und Subjektivität im Zeitalter ihrer strategischen Anerkennung, Bielefeld: transcript.

Eppel, R./Huemer, U./Mahringer, H./Weber, A./Knöller, J./Konle-Seidl, R. (2012): Öffentliche Arbeitsvermittlungssysteme und ihr Einfluss auf Suchverhalten und Erfolg der Arbeitssuche, Wien.

Esping-Andersen, G. (2004): »Die gute Gesellschaft und der neue Wohlfahrtsstaat«, in: Zeitschrift für Sozialreform, Jg. 50, Heft 1-2, S. 189-210.

Esping-Andersen, G. (1990): The three Worlds of Welfare Capitalism, Cambridge: Cambridge University Press.

Europäische Kommission (2007): Social activation: building the bridge between benefits and work. The EU Social Protection Social Inclusion Process Good Practice Article, 21.06.2007, o.O.

Europäische Kommission (1998): Die beschäftigungspolitischen Leitlinien für 1998. Entschließung des Rates vom 15.12.1997, Luxemburg.

eurostat (2013): Harmonisierte Arbeitslosenquote nach Geschlecht - Alter 1524, abgerufen am 20.03.2014 von http://epp.eurostat.ec.europa.eu/tgm/ta ble.do?tab=table \&plugin $=1 \&$ language $=$ de $\&$ pcode $=$ teilm 021 .

Fachhochschule Frankfurt am Main/infas-Institut für Angewandte Sozialwissenschaft/Wissenschaftszentrum Berlin (2009): »Implementations- und Governanceanalyse (Untersuchungsfeld 2)«, in: Ifo Schnelldienst, Jg. 62, Heft 1, S. 31-38.

Foucault, M. (2005a): »Die >Gouvernementalität〈 (Vortrag)«, in: D. Defert/F. Ewald (Hg.), Analytik der Macht, Frankfurt am Main: Suhrkamp, S. 148174.

Foucault, M. (2005b): »Die Machtverhältnisse gehen in das Innere der Körper über«, in: D. Defert/F. Ewald (Hg.), Analytik der Macht, Frankfurt am Main: Suhrkamp, S. 126-136.

Foucault, M. (2005c): Dits et Ecrits. Schriften, Band IV, 1980-1988, herausgegeben von D. Defert/F. Ewald, Frankfurt am Main: Suhrkamp.

Foucault, M. (2003): Dits et Ecrits. Schriften, Band III, 1976-1979, herausgegeben von D. Defert/F. Ewald, Frankfurt am Main: Suhrkamp.

Foucault, M. (2000): »Die Gouvernementalität«, in: U. Bröckling/S. Krasmann/T. Lemke (Hg.), Gouvernementalität der Gegenwart. Studien zur Ökonomisierung des Sozialen, Frankfurt am Main: Suhrkamp, S. 41-67.

Foucault, M. (1994): Überwachen und Strafen. Die Geburt des Gefängnisses, Frankfurt am Main: Suhrkamp.

Foucault, M. (1991): Die Ordnung des Diskurses, Frankfurt am Main: Fischer. 
Foucault, M. (1981): Archäologie des Wissens, Frankfurt am Main: Suhrkamp.

Foucault, M. (1976): Der Wille zum Wissen. Sexualität und Wahrheit 1, Frankfurt am Main: Suhrkamp.

Foucault, M. (1974): Die Ordnung der Dinge: Eine Archäologie der Humanwissenschaften, Frankfurt am Main: Suhrkamp.

Freier, C. (2013): »Zeitreise durch die Arbeitswelt. Kulturen der Arbeit im Wandel«, in: S. Brogi/C. Freier/U. Freier-Otten/K. Hartosch (Hg.), Repräsentationen von Arbeit. Transdisziplinäre Analysen und künstlerische Produktionen, Bielefeld: transcript, S. 35-58.

Freier, C. (2012): Qualitative Explorationsstudie zur Sozialen Aktivierung in der BRD. Forschungsbericht für das Institut für Arbeitsmarkt- und Berufsforschung der Bundesagentur für Arbeit (unveröffentlicht), Nürnberg.

Freier, C. (2011): „Grenzen aktivierender Sozialpolitik in der erodierenden Erwerbsgesellschaft«, in: M. Gubo/M. Kypta/F. Öchsner (Hg.), Kritische Perspektiven: >Turns`, Trends und Theorien, Berlin: LIT, S. 385-405.

Freier, C. (2008): Warum Menschen in Arbeit bringen? Deutungsmuster zur Arbeit und Grenzen externer Disziplinierung im Aktivierungsdiskurs am Beispiel der SGB II-Arbeitsgelegenheiten, unveröffentlichte Magisterarbeit, Erlangen.

Fromm, S./Sproß, C. (2008): Die Aktivierung erwerbsfähiger Hilfeempfänger: Programme, Teilnehmer, Effekte im internationalen Vergleich (= IABForschungsbericht 1/2008), Nürnberg, S 1-152.

Fuchs, V. J./Hummel, M./Hutter, C./Klinger, S./Wanger, S./Weber, E./Weigand, R./Zika, G. (2013): IAB-Prognose 2013/2014: Arbeitslosigkeit sinkt trotz Beschäftigungsrekord nur wenig (= IAB-Kurzbericht 18/2013), Nürnberg, S. $1-12$.

Fuchs-Heinritz, W. (1995): »Familie«, in: W. Fuchs-Heinritz/R. Lautmann/O. Rammstedt/H. Wienold (Hg.), Lexikon zur Soziologie, Opladen: Westdeutscher Verlag, S. 197.

Glaser, B. G./Strauss, A. L. (2005): Strategien qualitativer Forschung, Bern: Huber.

Globisch, C. (2012): »Strukturwandel sozialpolitischer Steuerung?«, in: Österreichische Zeitschrift für Soziologie, Jg. 37, Heft 1, S. 133-154.

Grabka, M. M./Westermeier, C. (2014): Anhaltend hohe Vermögensungleichheit in Deutschland (= DIW Wochenbericht Vermögensverteilung 9/2014), Berlin, S. 151-164.

Grimm, V. N./Hirseland, A./Vogel, B. (2013): »Die Ausweitung der Zwischenzone«, in: Soziale Welt, Jg. 64, S. 249-268. 
Harrach, E.-M./Loer, T./Schmidtke, O. (2000): Verwaltung des Sozialen : Formen der subjektiven Bewältigung eines Strukturkonflikts, Konstanz: UVK.

Hartz-Kommission - Kommission >Moderne Dienstleistungen am Arbeitsmarkt (2002): Bericht der Kommission, >Moderne Dienstleistungen am Arbeitsmarktı, Broschüre Nr. A 306, Berlin.

Hassel, A./Schiller, C. (2010): Die politische Dynamik von Arbeitsmarktreformen in Deutschland am Beispiel der Hartz IV-Reform, Forschungsbericht, o.O.

Helmut, A./Fertig, M./Koch, S./Osiander, C. (2011): Beschäftigungschancen von Ein-Euro-Jobbern in Hamburg. Der Träger macht den Unterschied (= IABKurzbericht 20/2011), Nürnberg, S. 1-8.

Henkel, D./Henke, J./Nägele, B./Pagels, N./Wagner, A. (2009): Erhebung von Ansätzen guter Praxis zur Integration Suchtkranker ins Erwerbsleben im Rahmen des SGB II. Abschlussbericht, Berlin.

Hielscher, V./Ochs, P. (2009): Arbeitslose als Kunden? Beratungsgespräche in der Arbeitsvermittlung zwischen Druck und Dialog, Berlin: edition sigma.

Hill, P. B./Kopp, J. (2013): Familiensoziologie. Grundlagen und theoretische Perspektiven, Wiesbaden: VS.

Hofmann, B./Koch, S./Kupka, P./Rauch, A./Schreyer, F./Stops, M./Wolff, J./Zahradnik, F. (2011): Wirkung und Nutzen von Sanktionen in der Grundsicherung. Zur Stärkung der Rechte von Arbeitslosen (= IAB-Stellungnahme 5/2011), Nürnberg, S. 1-17.

Hohendanner, C./Klemm, M./Promberger, M./Sowa, F. (2010): »Vom Ein-EuroJobber zum >regulären Mitarbeiter? Eine Mixed-Methods-Evaluation von Klebeeffekten der Arbeitsgelegenheiten mit Mehraufwandsentschädigung«, in: Zeitschrift für Evaluation, Jg. 9, Heft 2, S. 257-276.

Hohendanner, C./Walwei, U. (2013): »Arbeitsmarkteffekte atypischer Beschäftigung«, in: WSI-Mitteilungen, Jg. 66, Heft 4, S. 239-246.

Hohmeyer, K./Wolff, J. (2010): Wirkungen von Ein-Euro-Jobs für ALG II-Bezieher: Macht die Dosis einen Unterschied? (= IAB-Kurzbericht 4/2010), Nürnberg, S. 1-8.

Hüther, M./Scharnagel, B. (2005): »Die Agenda 2010: Eine wirtschaftspolitische Bilanz«, in: Aus Politik und Zeitgeschichte, Jg. 32-33, S. 23-30.

IAB - Institut für Arbeitsmarkt- und Berufsforschung der Bundesagentur für Arbeit (2013): Jahresplanung des IAB. Forschungs- und Arbeitsprogramm 2014, Nürnberg, abgerufen am 13.05.2014 von http://doku.iab.de/grauepap/ 2014/Forschungsprogramm_2014.pdf.

Jahn, E./Weber, E. (2013): Zeitarbeit: Zusätzliche Jobs, aber auch Verdrängung (= IAB-Kurzbericht 2/2013), Nürnberg, S. 1-6. 
Kantel, D. H. (2008): Grundsicherungsarbeit. Armuts- und Arbeitsmarktpolitik nach Hartz IV, Wiesbaden: VS.

Kantel, D. H. (2004): »Arbeit und soziale Sicherung in der Bürgergesellschaft«, in: B. Frevel (Hg.), Herausforderung demografischer Wandel, Wiesbaden: VS, S. 208-220.

Kantel, D. H. (2002a): »Die repressive Pädagogik des aktivierenden Sozialstaats - das Beispiel Arbeitslosigkeit«, in: Theorie und Praxis der sozialen Arbeit, Jg. 53, Heft1, S. 4-12.

Kantel, D. H. (2002b): »Mit Beschäftigungsgesellschaften gegen Arbeitslosigkeit?«, in: Sozialer Fortschritt, Jg. 51, Heft 5, S.123-130.

Kaltenborn, B./Kaps, P. (2013): Forschungsbericht Arbeitsmarkt 434. Einbeziehung der kommunalen Leistungen in die Zielsteuerung des SGB II, Bonn.

Kassel, E. (2010): Dialog Einkauf der Bundesagentur für Arbeit und Bildungsträger am Beispiel des AK Berufliche Bildung der Diakonie Württemberg Dialog mit dem REZ AK - Berufliche Bildung, Onlinedokument, abgerufen am 13.11.2013 von http://www.erev.de/auto/Downloads/Skripte_2010/ 50_2010/EinkaufDialog_mit_dem_REZ.pdf.

Kaufmann, F.-X. (2003): Varianten des Wohlfahrtsstaates. Der deutsche Sozialstaat im internationalen Vergleich, Frankfurt am Main: Suhrkamp.

Kelle, U. (1994): Empirisch begründete Theoriebildung: zur Logik und Methodologie interpretativer Sozialforschung, Weinheim: Deutscher Studien Verlag.

Kelle, U./Kluge, S. (2010a): »Regeln für Fallkontrastierung, Fallvergleich und empirisch begründete Typenbildung«, in: U. Kelle/S. Kluge (Hg.), Vom Einzelfall zum Typus. Fallvergleich und Fallkontrastierung in der qualitativen Sozialforschung (2. überarbeitete Auflage), Wiesbaden: VS, S. 108-112.

Kelle, U./Kluge, S. (2010b): Vom Einzelfall zum Typus. Fallvergleich und Fallkontrastierung in der qualitativen Sozialforschung (2. überarbeitete Auflage), Wiesbaden: VS.

Keller, R. (2013a): »Kommunikative Konstruktion und diskursive Konstruktion«, in: R. Keller/J. Reichertz/H. Knoblauch (Hg.), Kommunikativer Konstruktivismus. Theoretische und empirische Arbeiten $\mathrm{zu}$ einem neuen wissenssoziologischen Ansatz, Wiesbaden: VS, S. 69-94.

Keller, R. (2013b): »Zur Praxis der Wissenssoziologischen Diskursanalyse «, in: R. Keller/I. Truschkat (Hg.), Methodologie und Praxis der Wissenssoziologischen Diskursanalyse, Wiesbaden: VS, S. 27-68.

Keller, R. (2011): Diskursforschung. Eine Einführung für SozialwissenschaftlerInnen, Wiesbaden: VS. 
Keller, R. (2008): Wissenssoziologische Diskursanalyse. Grundlegung eines Forschungsprogramms, Wiesbaden: VS.

Keller, R. (2006): »Wissenssoziologische Diskursanalyse«, in: R. Keller/A. Hirseland/W. Schneider/W. Viehöver (Hg.), Handbuch Sozialwissenschaftliche Diskursanalyse: Theorien und Methoden, Wiesbaden: VS, S. 115-146.

Kessl, F. (2006): „Soziale Arbeit als Regierung - eine machtanalytische Perspektive«, in: S. Weber/S. Maurer (Hg.), Gouvernementalität und Erziehungswissenschaft. Wissen, Macht, Transformation, Wiesbaden: VS, S. 63-75.

Klinger, S./Rothe, T./Weber, E. (2013): Makroökonomische Perspektive auf die Hartz-Reformen: Die Vorteile überwiegen (= IAB-Kurzbericht 11/2013), Nürnberg, S. 1-8.

Knoblauch, H. (2005): Wissenssoziologie, Konstanz: UVK.

Knuth, M./Niewerth, C./Stegmann, T./Zink, L./Boockmann, B./Brändle, T./Kirchmann, A./Kleimann, R./Klempt, C./Langkabel, H. (2012): Evaluation der Zweiten Phase des Bundesprogramms >Perspektive 50plus - Beschäftigungspakte für Ältere in den Regionen`(2008-2010), Duisburg/Essen, S. 1-322.

Koch, S./Fertig, M. (2012): Evaluation von Arbeitsgelegenheiten in der Mehraufwandsvariante im Jobcenter München (= IAB-Forschungsbericht 1/2012), Nürnberg, S. 1-70.

Koch, S./Kupka, P. (2012): Öffentlich geförderte Beschäftigung. Integration und Teilhabe für Langzeitarbeitslose. Expertise im Auftrag der Abteilung Wirtschafts- und Sozialpolitik der Friedrich-Ebert-Stiftung (=WISO-Diskurs, Gesprächskreis Arbeit und Qualifizierung), Bonn.

Koch, S./Kupka, P./Steinke, J. (2009): Aktivierung, Erwerbstätigkeit und Teilhabe: vier Jahre Grundsicherung für Arbeitsuchende, Bielefeld: Bertelsmann.

Koenig, C./Haratsch, A. (2003): »Grundzüge des deutschen und des europäischen Vergaberechts«, in: NJW, Heft 37, S. 2637-2642.

Kolbe, C. (2012): »Irritationen im Zwangskontext - Interaktionen im SGB II«, in: WSI-Mitteilungen, Jg. 3, S. 198-204.

Konle-Seidl, R./Eichhorst, W. (2008a): »Does activation work?«, in: W. Eichhorst/O. Kaufmann/R. Konle-Seidl (Hg.), Bringing the jobless into work? Experiences with activation schemes in Europe and the US, Berlin/Heidelberg: VS, S. 415-443.

Konle-Seidl, R./Eichhorst, W. (2008b): Erwerbslosigkeit, Aktivierung und soziale Ausgrenzung. Deutschland im internationalen Vergleich. Gutachten im Auftrag der Friedrich-Ebert-Stiftung (=WISO-Diskurs, Gesprächskreis Arbeit und Qualifizierung), Bonn. 
Konle-Seidl, R./Lang, K. (2006): »Erwerbsfähige Transferbezieher im internationalen Vergleich. Von der Ausgliederung zur Integration in den Arbeitsmarkt «, in: Bundesarbeitsblatt, Heft 10, S. 14-22.

Kratz, D. (2013a): Entfremdete Hilfe. Biographien Langzeitarbeitsloser zwischen entgrenzter Lebensbewältigung und professioneller Beschäftigungsförderung, Dissertation, Neubiberg, abgerufen am 04.01.2014 von http://athene. bibl.unibw-muenchen.de:8081/node?id=90731

Kratz, D. (2013b): »Hilfe als Konflikt. Biographieanalytische Perspektiven auf die Gestaltung von Hilfeprozessen im Kontext der Arbeitsvermittlung«, in: Soziale Passagen, Jg. 5, Heft 1, S. 59-76.

Lefort, R./Foucault, M. (2005): »Michel Foucault: die Sicherheit und der Staat«, in: D. Defert/F. Ewald (Hg.), Analytik der Macht, Frankfurt am Main: Suhrkamp, S. 137-143.

Lemke, T./Krasmann, S./Bröckling, U. (2000): »Gouvernementalität, Neoliberalismus und Selbsttechnologien. Eine Einleitung«, in: U. Bröckling/S. Krasmann/T. Lemke (Hg.), Gouvernementalität der Gegenwart. Studien zur Ökonomisierung des Sozialen, Frankfurt am Main: Suhrkamp, S. 7-40.

Lessenich, S. (2012a): »Das Anerkennungsdefizitsyndrom des Wohlfahrtsstaats«, in: Österreichische Zeitschrift für Soziologie, Jg. 37, Heft 1, S. 99115.

Lessenich, S. (2012b): »Der Sozialstaat als Erziehungsagentur«, in: Aus Politik und Zeitgeschichte, Jg. 49-50, S. 55-61.

Lessenich, S. (2009a): »Aktivierungspolitik und Anerkennungsökonomie«, in: Soziale Passagen, Jg. 1, Heft 2, S. 163-176.

Lessenich, S. (2009b): Die Neuerfindung des Sozialen. Der Sozialstaat im flexiblen Kapitalismus, Bielefeld: transcript.

Lessenich, S. (2009c): »Krise des Sozialen?«, in: Aus Politik und Zeitgeschichte, Jg. 52, S. 28-34.

Lessenich, S. (2003): »Soziale Subjektivität. Die neue Regierung der Gesellschaft«, in: Mittelweg 36, Jg. 12, Heft 4, S. 80-93.

Liebold, R./Trinczek, R. (2009): »Experteninterview«, in: S. Kühl/P. Strodtholz/A. Taffertshofer (Hg.), Handbuch Methoden der Organisationsforschung. Quantitative und Qualitative Methoden, Wiesbaden: VS, S. 32-56.

Lipsky, M. (1980): Street Level Bureaucracy: Dilemmas of the Individual in Public Services, New York: Russel Sage Foundation.

Ludwig-Mayerhofer, W. (2012): »Arbeitsmarkt. Für alle wichtig, für viele unsicherer«, in: S. Hradil (Hg.), Deutsche Verhältnisse. Eine Sozialkunde, Bonn, S. 289-312. 
Ludwig-Mayerhofer, W. (2005): »Arbeitslosigkeit«, in: M. Abraham/T. Hinz (Hg.), Arbeitsmarktsoziologie. Probleme, Theorien, empirische Befunde, Wiesbaden: VS, S. 199-239.

Ludwig-Mayerhofer, W./Behrend, O./Sondermann, A. (2009): Auf der Suche nach der verlorenen Arbeit Arbeitslose und Arbeitsvermittler im neuen Arbeitsmarktregime, Konstanz: UVK.

Ludwig-Mayerhofer, W./Promberger, M. (2008): »Einleitung: Qualitative Forschung zur Reform der Arbeitsmarktpolitik«, in: Zeitschrift für Sozialreform, Jg. 54, Heft 1, S. 3-10.

Ludwig-Mayerhofer, W./Sondermann, A. (2012): »Fördern und Fordern ... aber wen eigentlich?«, in: M. Bereswill/C. Figlestahler/L. Y. Haller/M. Perels/F. Zahradnik (Hg.), Wechselverhältnisse im Wohlfahrtsstaat. Dynamiken gesellschaftlicher Justierungsprozesse, Münster: Westfälisches Dampfboot, S. 194-211.

Lutz, T. (2010): Soziale Arbeit im Kontrolldiskurs Jugendhilfe und ihre Akteure in postwohlfahrtstaatlichen Gesellschaften, Wiesbaden: VS.

Magnin, C. (2006): »Die Bürokratisierung des Arbeitsmarktes. Zu den Paradoxien der aktivierungspolitischen Bearbeitung von Arbeitslosigkeit«, in: Sozialer Sinn, Heft 1, S. 79-106.

Maier, K. (Hg.) (2008): Soziale Arbeit in der `Krise der Arbeitsgesellschaft‘, Frankfurt am Main: FEL.

Marshall, T. (1992): Bürgerrecht und soziale Klassen: zur Soziologie des Wohlfahrtsstaates, Frankfurt am Main: Campus.

Marx, K. (1968): Das Kapital. Kritik der politischen Ökonomie, Bd.1: Der Produktionsprozeß des Kapitals (= MEW, Bd. 23), Berlin: Dietz, S. 11-802.

Maurer, S. (2006): »Soziale Arbeit und soziale Bewegungen als (kollektive) Akteure >beweglicher Ordnungen «", in: S. Weber/S. Maurer (Hg.), Gouvernementalität und Erziehungswissenschaft. Wissen, Macht, Transformation, Wiesbaden: VS, S. 233-252.

Meinefeld, W. (2005): »Hypothesen und Vorwissen in der qualitativen Sozialforschung «, in: U. Flick/E. von Kardoff/I. Steinke (Hg.), Qualitative Forschung. Ein Handbuch (4. überarbeitete Auflage), Reinbek bei Hamburg: Rowohlt, S. 265-275.

Meuser, M./Nagel, U. (1991): ExpertInneninterviews - vielfach erprobt, wenig bedacht. Ein Beitrag zur qualitativen Methodendiskussion, in: D. Garz, K. Kraimer (Hg.), Qualitativ-empirische Sozialforschung. Konzepte, Methoden, Analysen, Opladen: Westdeutscher Verlag, S. 441-471.

Meuser, M./Sackmann, R. (Hg.) (1992): Analyse sozialer Deutungsmuster, Pfaffenweiler: Centaurus. 
Musner, L. (2008): »Prekarisierung und Überforderung. Leben jenseits der Gesellschaft«, in: R. Lindner/L. Musner (Hg.), Unterschicht. Kulturwissenschaftliche Erkundungen der "Armen" in Geschichte und Gegenwart (= Edition Parabasen, Band 8), Freiburg im Breisgau/Berlin/Wien: Rombach, S. 79-96.

Münch, R. (2009): Das Regime des liberalen Kapitalismus. Inklusion und Exklusion im neuen Wohlfahrtsstaat, Frankfurt am Main/New York: Campus.

Murray, C. (1996): Charles Murray and the Underclass: The Developing Debate, herausgegeben von R. Lister, London: Hartington Fine Arts.

Nullmeier, F. (2011): »Output-Steuerung und Performance Measurement«, in: B. Blanke/S. von Bandemer/F. Nullmeier/G. Wewer (Hg.), Handbuch zur Verwaltungsreform, Wiesbaden: VS, S. 465-475.

Öchsner, T. (2013): »Spartipps für Hartz-IV-Empfänger >Gehen Sie nie hungrig einkaufen«", in: Süddeutsche Zeitung vom 18.07.2013, abgerufen am 18.07.2013 von http:/www.sueddeutsche.de/wirtschaft/spartipps-fuer-hartziv-empfaenger-gehen-sie-nie-hungrig-einkaufen-1.1724883.

Öchsner, T. (2011): »Langzeitarbeitslosigkeit - Einmal Hartz IV, immer Hartz IV «, in: Süddeutsche Zeitung vom 15.09.2011, abgerufen am 15.11.2011 von http://www.sueddeutsche.de/wirtschaft/langzeitarbeitslosigkeit-einmal-hartziv-immer-hartz-iv-1.1143841.

Opielka, M. (2003): »Aktivierung durch Verpflichtung ? Von der Pflicht zur Erwerbsarbeit zur Idee eines Sozialdienstes«, in: vorgänge, Jg. 164, Heft 4, S. $113-120$.

Opielka, M. (2004): Sozialpolitik. Grundlagen und vergleichende Perspektiven, Reinbek bei Hamburg: Rowohlt.

Oschmiansky, F. (2011): Die öffentliche Arbeitsvermittlung, herausgegeben von der Bundeszentrale für politische Bildung, abgerufen am 18.12.2013 von http://www.bpb.de/politik/innenpolitik/arbeitsmarktpolitik/55132/oeffentlich e-arbeitsvermittlung? $\mathrm{p}=0$.

Oschmiansky, F. (2010): Folgen der Arbeitslosigkeit (= Dossier Arbeitsmarktpolitik), herausgegeben von der Bundeszentrale für politische Bildung, abgerufen am 07.01.2014 von http://www.bpb.de/politik/innenpolitik/ arbeitsmarktpolitik/54992/folgen-der-arbeitslosigkeit.

Oschmiansky, F./Ebach, M. (2009): »Vom AFG 1969 zur Instrumentenreform 2009: Der Wandel des arbeitsmarktpolitischen Instrumentariums«, in: S. Bothfeld/W. Sesselmeier/C. Bogedan (Hg.), Arbeitsmarktpolitik in der sozialen Marktwirtschaft. Vom Arbeitsförderungsgesetz zum Sozialgesetzbuch II und III, Wiesbaden: VS , S. 79-93. 
Peuckert, R. (2002): »Lektion VI. Abweichendes Verhalten und soziale Kontrolle«, in: H. Korte/B. Schäfers (Hg.), Einführung in Hauptbegriffe der Soziologie, Opladen: Leske und Budrich, S. 105-125.

Plankensteiner, A. (2013): Aktivierende Sozialstaatlichkeit und das Praxisfeld der Erziehungshilfen, Weinheim/Basel: Beltz Juventa.

Polanyi, K. (1977): The Great Transformation. Politische und Ökonomische Ursprünge von Gesellschaften und Wirtschaftssystemen, Wien: Europaverlag.

Pongratz, H. J./Voß, G. G. (2004): Typisch Arbeitskraftunternehmer? Befunde der empirischen Arbeitsforschung, Berlin: edition sigma.

Pongratz, H. J./Voß, G. G. (2003): Arbeitskraftunternehmer. Erwerbsorientierungen in entgrenzten Arbeitsformen, Berlin: edition sigma.

Preisendörfer, Peter (2011): Organisationssoziologie. Grundlagen, Theorien und Problemstellungen, Wiesbaden: VS.

Presse- und Informationsamt der Bundesregierung (Hg.) (2003): Agenda 2010. Deutschland bewegt sich. Antworten zur Agenda 2010, Berlin.

Promberger, M. (2009): »Exkurs: Das SGB II: Kontinuität oder Bruch in der deutschen Sozialpolitik?«, in: S. Koch/P. Kupka/J. Steinke (Hg.), Aktivierung, Erwerbstätigkeit und Teilhabe. Vier Jahre Grundsicherung für Arbeitsuchende (= IAB-Bibliothek 315/2009), Bielefeld: Bertelsmann, S. 22-29.

Promberger, M. (2005): »Eine kurze Geschichte der Arbeitslosigkeit. Teil 3: Von der Ära des Wirtschaftswunders bis zum Jahr 2000«, in: Arbeit und Beruf, Jg. 56, Heft 3, S. 65-67.

Promberger, M./Wenzel, U./Pfeiffer, S./Hacket, A./Hirseland, A. (2008): »Beschäftigungsfähigkeit, Arbeitsvermögen und Arbeitslosigkeit«, in: WSIMitteilungen, Heft 2, S. 70-76.

Raithel, J. (2006): »Beratung. Im Spannungsfeld zwischen Anspruch und Wirklichkeit«, in: B. Dollinger/J. Raithel (Hg.), Aktivierende Sozialpädagogik. Ein kritisches Glossar, Wiesbaden: VS, S. 41-50.

Rappaport, J. (1985): »Ein Plädoyer für die Widersprüchlichkeit. Ein sozialpolitisches Konzept des »empowerment« anstelle präventiver Ansätze«, in: Verhaltenstherapie und psychosoziale Praxis, Jg. 17, Heft 2, S. 257-278.

Rat der EU (1997): Die beschäftigungspolitischen Leitlinien für 1998, o.O.

Rhein, T. (2013): Erwerbseinkommen: Deutsche Geringverdiener im europäischen Vergleich (= IAB-Kurzbericht 15/2013), Nürnberg, S. 1-10.

Ruoff, M. (2007): Foucault-Lexikon, Paderborn: Wilhelm Fink.

Schallberger, P./Wyer, B. (2010): Praxis der Aktivierung. Eine Untersuchung von Programmen zur vorübergehenden Beschäftigung, Konstanz: UVK.

Schedler, K./Proeller, I. (2011): New Public Management (5. Auflage), Bern/ Stuttgart/Wien: UTB. 
Schmid, G. (2012): »Annäherung an eine Politik der Vollbeschäftigung in Europa«, in: Aus Politik und Zeitgeschichte, Jg. 62, Heft 14-15, S. 53-61.

Schmid, J. (2012): Probleme und Zukunftsperspektiven des Sozialstaates (= Dossier Deutsche Verhältnisse. Eine Sozialkunde), herausgeben von der Bundeszentrale für politische Bildung, aufgerufen am 21.03.2014 von http:// www.bpb.de/politik/grundfragen/deutsche-verhaeltnisse-eine-sozialkunde/ 138845/probleme-und-zukunftsperspektiven-des-sozialstaates?p=all.

Schmuhl, H.-W. (2003): Arbeitsmarktpolitik und Arbeitsverwaltung in Deutschland 1871-2002. Zwischen Vorsorge, Hoheit und Markt, (= Beiträge zur Arbeitsmarkt- und Berufsforschung 270), Nürnberg.

Schönig, W. (2006): »Aktivierungspolitik. Eine sozialpolitische Strategie und ihre Ambivalenz für soziale Dienste und praxisorientierte Forschung«, in: B. Dollinger/J. Raithel (Hg.), Aktivierende Sozialpädagogik. Ein kritisches Glossar, Wiesbaden: VS, S. 23-39.

Schönig, W. (2002): »Institutionelle Perspektiven der Bundesanstalt für Arbeit Maßgeschneiderte Betreuung durch das neue Steuerungsmodell?«, in: Sozialer Fortschritt, Jg. 51, Heft 1, S. 9-12.

Schubert, M./Parthier, K./Kupka, P./Krüger, U./Holke, J./Fuchs, P. (2013): Menschen mit psychischen Störungen im SGB II (= IAB-Forschungsbericht 12/2013), Nürnberg, S. 1-104.

Schulz, A. (2010): Organisationen zwischen Markt, Staat und Zivilgesellschaft. Arbeitsmarktförderung von Langzeitarbeitslosen im Deutschen Caritasverband, Wiesbaden: VS.

Schütz, H. (2012): »Neue und alte Regelsteuerung in der deutschen Arbeitsverwaltung«, in: S. Bothfeld/W. Sesselmeier/C. Bogedan (Hg.), Arbeitsmarktpolitik in der sozialen Marktwirtschaft. Vom Arbeitsförderungsgesetz zu Sozialgesetzbuch II und III, Wiesbaden: VS.

Schütz, H./Steinwede, J./Schröder, H./Kaltenborn, B./Wielage, N./Christe, G./Kupka, P. (2011): Vermittlung und Beratung in der Praxis. Eine Analyse von Dienstleistungsprozessen am Arbeitsmarkt (=IAB-Bibliothek 330/2011), Nürnberg, S. 1-331.

Sell, S. (2006): Modernisierung und Professionalisierung der Arbeitsvermittlung. Strategien, Konzepte und Modelle unter Berücksichtigung internationaler Erfahrungen. Gutachten der Friedrich-Ebert-Stiftung, Bonn.

Sennett, R. (2000): Der flexible Mensch: die Kultur des neuen Kapitalismus (4. Auflage), München: Siedler.

Sondermann, A. (2013): »Zwischen subjektiven Bezügen zu Erwerbsarbeit und den Normen der Arbeitsmarktpolitik. Erwerbslose in einem schwierigen Spannungsfeld«, in: S. Brogi/C. Freier/U. Freier-Otten/K. Hartosch (Hg.), 
Repräsentationen von Arbeit. Transdisziplinäre Analysen und künstlerische Analysen, Bielefeld: transcript, S. 231-246.

Sowa, F./Klemm, M./Freier, C. (2012): >Ein-Euro-Jobs $<$ in Deutschland: Qualitative Fallstudien zur Auswirkung der SGB-II-Arbeitsgelegenheiten auf Beschäftigungsstruktur und Arbeitsmarktverhalten von Organisationen (= IABForschungsbericht 15/2012), Nürnberg, S.1-140.

Sowa, F./Staples, R. (2013): »Re-Präsentation eines idealen Arbeitsmarktes in Beratungsgesprächen der Bundesagentur für Arbeit«, in: S. Brogi/C. Freier/U. Freier-Otten/K. Hartosch (Hg.), Repräsentationen von Arbeit. Transdisziplinäre Analysen und künstlerische Analysen, Bielefeld: transcript, S. 247-264.

Srubar, I. (2006): »Wo liegt Macht? Zur Semantik und Sinnbildung in der Politik«, Onlineveröffentlichung, abgerufen am 23.05.2008 von http://www.politik-kultur-religion.de/veranstaltung.

Staples, R./Trinczek, R./Whittall, M. (2013): »>Posted Workers $:$ Zwischen Regulierung und Invisibilisierung«, in: Arbeit, Jg. 22, Heft 4, S. 271-286.

Statistisches Bundesamt (2013): Statistisches Jahrbuch 2013, Wiesbaden.

Steinke, J./Koch, S./Kupka, P./Osiander, C./Dony, E./Güttler, D./Hesse, C./Knapp, B. (2012): Neuorientierung der Arbeitsmarktpolitik. Die Neuausrichtung der arbeitsmarktpolitischen Instrumente aus dem Jahr 2009 im Blickpunkt: Mehr Flexibilität und größere Handlungsspielräume für die Vermittler? (= IAB-Forschungsbericht 2/2012), Nürnberg.

Strübing, J. (2008): Grounded Theory. Zur sozialtheoretischen und epistemologischen Fundierung des Verfahrens der empirisch begründeten Theoriebildung (2. überarbeitete Auflage), Wiesbaden: VS.

Tisch, A. (2010): Kundenzufriedenheit im SGB II: Arbeitsvermittler im Urteil der ALG-II-Empfänger (= IAB-Kurzbericht 7/2010), S. 1-8.

Trinczek, R. (2013): »Veränderungen im Berufsbildungssystem in Deutschland und Varieties of Capitalism«, in: A. Riedl/R. Tenberg (Hg.), Berufspädagogische Praxis in wissenschaftlicher Reflexion. Jubiläumsband für Andreas Schelten, Stuttgart: Franz Steiner, S. 203-215.

Trinczek, R. (2011): »Überlegungen zum Wandel von Arbeit«, in: WSIMitteilungen, Jg. 64, Heft 11, S. 606-614.

Vobruba, G. (2009): »Effizienz durch Sicherheit. Soziale Rahmenbedingungen moderner kapitalistischer Ökonomien«, in: H. Seifert/O. Struck (Hg.), Arbeitsmarkt und Sozialpolitik: Kontroversen um Effizienz und soziale Sicherheit, Wiesbaden: VS, S. 17-27.

Vobruba, G. (2007): Entkoppelung von Arbeit und Einkommen. Das Grundeinkommen in der Arbeitsgesellschaft (2. erweiterte Auflage), Wiesbaden: VS. 
Walwei, U. (2009): »Arbeitslosenversicherung in der Wirtschaftskrise«, in: $\mathrm{G}+\mathrm{S}$ Gesundheits- und Sozialpolitik, Jg. 5, Heft 12, S. 10-17.

Weber, M. (2005): Wirtschaft und Gesellschaft, Neu Isenburg: Melzer.

Weyand, J./Düwell, F. J. (2005): Das neue Arbeitsrecht. Hartz-Gesetze und Agenda 2010 in der arbeits- und sozialrechtlichen Praxis, Baden-Baden: Nomos.

Wolf, Michael (2006): »Hartz IV: ausgrenzende Aktivierung oder Lehrstück über die Antastbarkeit der Würde des Menschen«, in: Utopie kreativ, Heft 194, S. 1079-1095.

Zimmermann, B. (2006): Arbeitslosigkeit in Deutschland: zur Entstehung einer sozialen Kategorie, Frankfurt: Campus. 


\section{Anhang}

\subsection{ABKÜRZUNGSVERZEICHNIS}

$\begin{array}{ll}\text { AA } & \text { Agentur für Arbeit (ehemaliges Arbeitsamt) } \\ \text { ABM } & \text { Arbeitsbeschaffungsmaßnahme } \\ \text { AFG } & \text { Arbeitsförderungsgesetz } \\ \text { AGH } & \text { Arbeitsgelegenheit } \\ \text { Alg } & \text { Arbeitslosengeld } \\ \text { Alg II } & \text { Arbeitslosengeld II } \\ \text { ALH } & \text { Arbeitslosenhilfe } \\ \text { AM } & \text { Arbeitsmarkt } \\ \text { ARGE } & \text { Arbeitsgemeinschaft zwischen Arbeitsagentur und Kommune; vor } \\ & \text { Neuregelung 2010 typisches Trägerschaftsmodell der Grundsi- } \\ & \text { cherung, dann vom Jobcenter abgelöst } \\ \text { BA } & \text { Bundesagentur für Arbeit (bis 2002 Bundesanstalt für Arbeit) } \\ \text { BHO } & \text { Bundeshaushaltsordnung } \\ \text { BGB } & \text { Bürgerliches Gesetzbuch } \\ \text { BMAS } & \text { Bundesministerium für Arbeit und Soziales (bis 2007 für Arbeit } \\ & \text { und Sozialordnung) } \\ \text { BMFSFJ } & \text { Bundesministerium für Familie, Senioren, Frauen und Jugend } \\ \text { BMWA } & \text { Bundesministerium für Wirtschaft und Arbeit (existierte 2002 bis } \\ & \text { 2005) } \\ \text { BRH } & \text { Bundesrechnungshof } \\ \text { BSHG } & \text { Bundessozialhilfegesetz } \\ \text { BvB } & \text { Berufsvorbereitende Bildungsmaßnahme } \\ \text { BVerfG } & \text { Bundesverfassungsgericht } \\ \text { BvR } & \text { Registerzeichen bzw. Aktenzeichen des Bundesverfassungs- } \\ & \text { gerichts } \\ \text { DStGB } & \text { Deutscher Städte- und Gemeindebund }\end{array}$




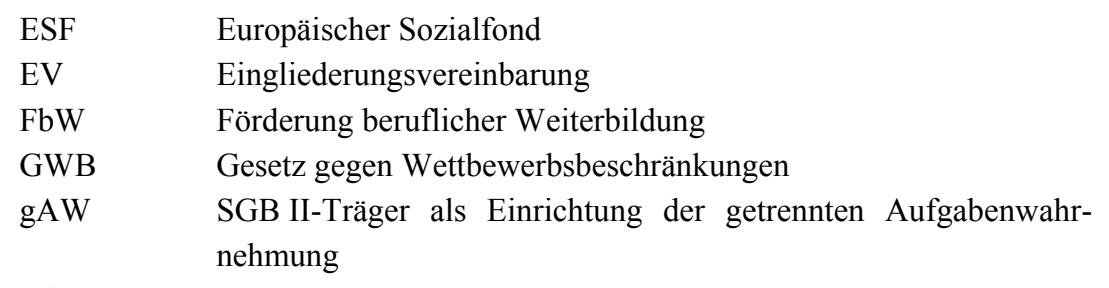

Job-AQTIV-

Gesetz Gesetz zur Reform der arbeitsmarktpolitischen Instrumente; AQTIV steht für »Aktivieren, Qualifizieren, Trainieren, Investieren, Vermitteln «

GWB Gesetz gegen Wettbewerbsbeschränkungen

HEGA Handlungsempfehlungen und Geschäftsanweisungen für Fachkräfte der SGB-II- und SGB-III-Träger

HGrG Gesetz über die Grundsätze des Haushaltsrechts des Bundes und der Länder

I KundInnen, die als integriert, aber weiterhin hilfebedürftig gelten; Kategorisierung von Sozialleistungsbeziehenden durch SGB-IITräger

IAB Institut für Arbeitsmarkt- und Berufsforschung der Bundesagentur für Arbeit

IF Integrationsferne KundInnen; Kategorisierung von Sozialleistungsbeziehenden durch SGB-II-Träger

IG KundInnen mit festgestelltem Stabilisierungsbedarf; Kategorisierung von Sozialleistungsbeziehenden durch SGB-II-Träger

IN KundInnen mit festgestellter Integrationsfähigkeit; Kategorisierung von Sozialleistungsbeziehenden durch SGB-II-Träger

i.V.m. in Verbindung mit

KdU Kosten der Unterkunft und Heizung, die von Kommunen übernommen werden

LHO Landeshaushaltsordnungen

MAE Mehraufwandsentschädigung, wird bei Arbeitsgelegenheiten an Maßnahmenteilnehmende gezahlt; bezeichnet in der Alltagssprache sowohl Ein-Euro-Jobber wie auch die Beschäftigungsmaßnahme selbst (Arbeitsgelegenheit mit Mehraufwandsentschädigung); in beiden Varianten wird Abkürzung auch in diesem Text verwandt

pAp SGB-II-Träger-Fachkräfte, sogenannte persönliche Ansprechpartner

RD Regionaldirektion 


$\begin{array}{ll}\text { Regio } & \text { Region } \\ \text { REZ } & \text { Regionale Einkaufszentren } \\ \text { SAM } & \text { Strukturanpassungsmaßnahmen } \\ \text { SGB } & \text { Sozialgesetzbuch } \\ \text { SGB II } & \text { Sozialgesetzbuch Zweites Buch - Grundsicherung für Arbeitssu- } \\ & \text { chende } \\ \text { SGB III } & \text { Sozialgesetzbuch Drittes Buch - Arbeitsförderung } \\ \text { SGB XII } & \text { Sozialgesetzbuch Zwölftes Buch - Sozialhilfe } \\ \text { VgV } & \text { Verordnung über die Vergabe öffentlicher Aufträge auch als Ver- } \\ & \text { dingungsordnung bezeichnet } \\ \text { VOB } & \text { Vergabe- und Vertragsordnung für Bauleistungen } \\ \text { VOL } & \text { Vergabe- und Vertragsordnung für Leistungen } \\ \text { VHS } & \text { Volkshochschule } \\ \text { WDA } & \text { Wissenssoziologische Diskursanalyse } \\ \text { ZDH } & \text { Zentralverband des Deutschen Handwerks } \\ \text { zkT } & \text { zugelassener kommunaler Träger (im Feld auch Optionskommune }\end{array}$




\section{2 ÜBERSICHT ÜBER DIE GEFÜHRTEN INTERVIEWS}

\begin{tabular}{|c|c|c|}
\hline Name & Einrichtung anonym & Person anonym \\
\hline Int01Regio01 & ARGE & $\begin{array}{l}\text { Fallmanagerin, Arbeits- } \\
\text { vermittler, Erstkontakt }\end{array}$ \\
\hline Int01Regio02 & Bildungsträger $\mathrm{GmbH}$ & $\begin{array}{l}\text { Leiterin und Sachbear- } \\
\text { beiterin }\end{array}$ \\
\hline Int02Regio02 & $\begin{array}{l}\text { Sozialpsychiatrischer Dienst } \\
\text { der Kommune }\end{array}$ & Sozialpädagogin \\
\hline Int03Regio02 & Kirchliche Beratungsstelle & Sozialpädagogin \\
\hline Int04Regio02 & $\begin{array}{l}\text { ARGE Maßnahmen- } \\
\text { koordination }\end{array}$ & $\begin{array}{l}\text { Teamleiterin Maßnah- } \\
\text { menbüro }\end{array}$ \\
\hline Int05Regio02 & $\begin{array}{l}\text { Religiöser sozialpsychiatri- } \\
\text { scher Dienst }\end{array}$ & $\begin{array}{l}\text { Leiterin und Sachbear- } \\
\text { beiterin }\end{array}$ \\
\hline Int06Regio02 & REZ & $\begin{array}{l}\text { Einkaufsexpertin, Pro- } \\
\text { duktbetreuerin }\end{array}$ \\
\hline Int07Regio02 & Bundesagentur & $\begin{array}{l}\text { Expertin Gesundheits- } \\
\text { orientierung }\end{array}$ \\
\hline Int01Regio04 & Jobcenter & Bereichsleiter \\
\hline Int02Regio04 & Jobcenter & $\begin{array}{l}\text { Fallmanagerin und Ar- } \\
\text { beitsvermittlerin }\end{array}$ \\
\hline Int03Regio04 & Christlicher Jugendträger & $\begin{array}{l}\text { Leiterin des Trägers, } \\
\text { Sozialpädagogin }\end{array}$ \\
\hline Int04Regio04 & $\begin{array}{c}\text { ARGE, zwei regionale Bil- } \\
\text { dungsträger }\end{array}$ & $\begin{array}{l}\text { Bereichsleiterin U25, } \\
\text { Arbeitsvermittlerin U25, } \\
\text { Fallmanagerin U25, drei } \\
\text { Vertreter zweier Träger }\end{array}$ \\
\hline Int01Regio05 & ARGE & $\begin{array}{l}\text { Teamleiterin, Sozialme- } \\
\text { diziner, Psychologin, }\end{array}$ \\
\hline
\end{tabular}




\begin{tabular}{|c|c|c|}
\hline & & Fallmanagerin \\
\hline Int02Regio05 & ARGE & Teamleiterin \\
\hline Int01Regio06 & ARGE & Maßnahmenbetreuer \\
\hline Int02Regio06 & ARGE Optionskommune & $\begin{array}{c}\text { Teamleiter, städtischer } \\
\text { Mitarbeiter, Fallmana- } \\
\text { gerin }\end{array}$ \\
\hline Int03Regio06 & Bundesweiter Bildungsträger & Mitarbeiter und Leiterin \\
\hline Int04Regio06 & $\begin{array}{l}\text { Kommunalreferat Options- } \\
\text { kommune }\end{array}$ & $\begin{array}{l}\text { Zwei kommunale Mit- } \\
\text { arbeiter, Leiterin }\end{array}$ \\
\hline Int05Regio06 & $\begin{array}{c}\text { Sozialbetrieblicher Maßnah- } \\
\text { menträger }\end{array}$ & Leiterin \\
\hline Int06Regio06 & $\begin{array}{l}\text { Familienorientierter Maß- } \\
\text { nahmenträger }\end{array}$ & $\begin{array}{l}\text { Geschäftsführerin, } \\
\text { Fachbereichsleiterin }\end{array}$ \\
\hline Int01Regio07 & Jobcenter & Fallmanagerin \\
\hline Int02Regio07 & Jobcenter & Fallmanagerin \\
\hline Int03Regio07 & Jobcenter & Zentr. Projektmanager \\
\hline Int04Regio07 & Bundesweiter Bildungsträger & Leitung \\
\hline Int01Regio10 & Jobcenter & Fallmanager \\
\hline Int02Regio10 & Jobcenter & Fallmanager \\
\hline Int03Regio10 & Regionaler Bildungsträger & Leitung \\
\hline Int01Regio11 & Regionalverwaltung & $\begin{array}{l}\text { LK-Mikroprojektbetreu- } \\
\text { erin, Jobcentermitarbei- } \\
\text { terin, Vertreterin des } \\
\text { Landestheaters }\end{array}$ \\
\hline Int02Regio11 & $\begin{array}{c}\text { Frauenbezogener Maßnah- } \\
\text { menträger }\end{array}$ & $\begin{array}{c}\text { Trägerleiterin, Teamlei- } \\
\text { terin }\end{array}$ \\
\hline
\end{tabular}




\begin{tabular}{|c|c|c|}
\hline Int03Regio11 & Jobcenter & Teamleiterin \\
\hline Int01Regio12 & Jobcenter & Teamleiterin \\
\hline Int02Regio12 & Jobcenter & Arbeitsvermittlerin \\
\hline Int03Regio12 & Jobcenter & $\begin{array}{l}\text { Markt- und Integrati- } \\
\text { onsexpertin }\end{array}$ \\
\hline Int04Regio12 & Bundesweiter Bildungsträger & Leitung \\
\hline Int01Regio13 & Optionskommune & $\begin{array}{l}\text { Leitung Projektma- } \\
\text { nagement }\end{array}$ \\
\hline Int02Regio13 & Optionskommune & Projektmanagement \\
\hline Int03Regio13 & Optionskommune & Fallmanagerin \\
\hline Int04Regio13 & Optionskommune & Fallmanagerin \\
\hline Int05Regio13 & Optionskommune & Fallmanagerin \\
\hline Int06Regio13 & Optionskommune & Projektmanagement \\
\hline Int07Regio13 & Bundesweiter Bildungsträger & Geschäftsführerin \\
\hline Int08Regio13 & Regionaler Bildungsträger & $\begin{array}{c}\text { Leiterin Berufsvorberei- } \\
\text { tung }\end{array}$ \\
\hline $\begin{array}{c}\text { Telefoninterview01 } \\
\text { Regio03 }\end{array}$ & Boxverein & Vereinsvorsitzende \\
\hline $\begin{array}{c}\text { Telefoninterview02 } \\
\text { Regio02 }\end{array}$ & $\begin{array}{c}\text { Psychologischer Dienst der } \\
\text { BA }\end{array}$ & Leitung \\
\hline Memo01Regio04 & $\begin{array}{l}\text { Gewaltprävention im Rah- } \\
\text { men eines Jugendhilfevereins }\end{array}$ & $\begin{array}{l}\text { Anti-Aggressions- } \\
\text { Trainer }\end{array}$ \\
\hline Int01Regio08 & & Wissenschaftler \\
\hline Int01Regio09 & & Wissenschaftler \\
\hline
\end{tabular}




\section{Gesellschaft der Unterschiede}

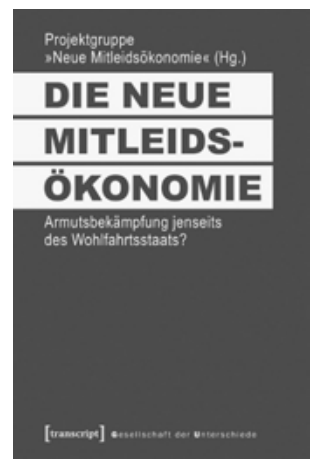

Projektgruppe »Neue Mitleidsökonomie« (Hg.)

Die neue Mitleidsökonomie

Armutsbekämpfung jenseits

des Wohlfahrtsstaats?

März 20I7, ca. 250 Seiten, kart., ca. 24,99€, ISBN 978-3-8376-3158-6

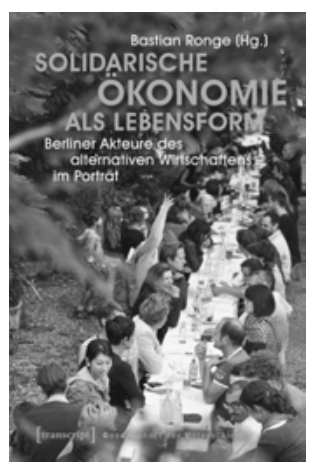

Bastian Ronge (Hg.)

Solidarische Ökonomie als Lebensform

Berliner Akteure des alternativen Wirtschaftens im Porträt

Oktober 2016, I44 Seiten, kart., I8,99 €, ISBN 978-3-8376-3662-8

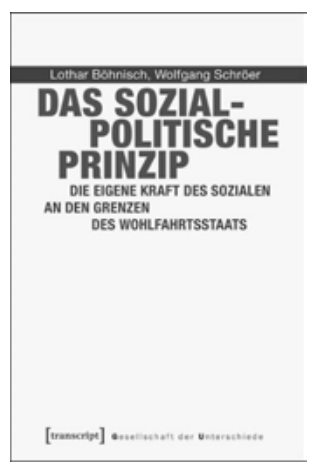

Lothar Böhnisch, Wolfgang Schröer

Das Sozialpolitische Prinzip

Die eigene Kraft des Sozialen an den Grenzen des Wohlfahrtsstaats

Oktober 20I6, I84 Seiten, kart., 29,99€, ISBN 978-3-8376-3459-4 


\section{Gesellschaft der Unterschiede}

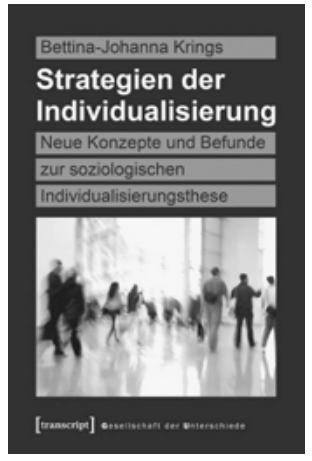

Bettina-Johanna Krings

Strategien der Individualisierung

Neue Konzepte und Befunde zur soziologischen

Individualisierungsthese

August 20I6, 304 Seiten, kart., 34,99€, ISBN 978-3-8376-3347-4

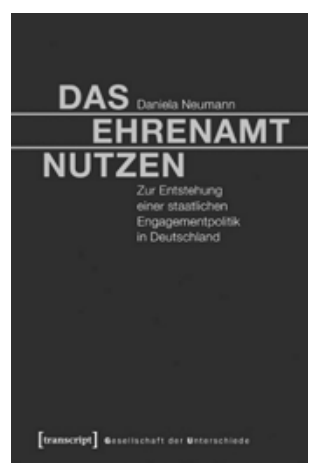

Daniela Neumann

Das Ehrenamt nutzen

Zur Entstehung einer staatlichen

Engagementpolitik in Deutschland

Februar 20I6, 508 Seiten, kart., 39,99€, ISBN 978-3-8376-3278-I

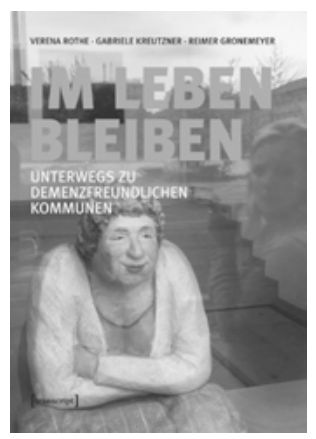

Verena Rothe, Gabriele Kreutzner,

Reimer Gronemeyer

Im Leben bleiben

Unterwegs zu Demenzfreundlichen Kommunen

20I5, 288 Seiten, kart., 24,99€,

ISBN 978-3-8376-2996-5 


\section{Gesellschaft der Unterschiede}

Johanna Muckenhuber, Josef Hödl, Martin Griesbacher (Hg.)

Normalarbeit

Nur Vergangenheit

oder auch Zukunft?

Dezember 20I6, ca. 300 Seiten, kart., 29,99 €, ISBN 978-3-8376-3462-4

Christina Goesmann

Wertschätzung ehrenamtlicher Arbeit Quellen der Wertschätzung in der psychosozialen Demenzbetreuung November 20I6, 218 Seiten, kart., 32,99€, ISBN 978-3-8376-3668-o

Claudia Stöckl,

Karin Kicker-Frisinghelli, Susanna Finker (Hg.)

Die Gesellschaft des langen Lebens

Soziale und individuelle

Herausforderungen

November 2016, 284 Seiten, kart., 34,99 €, ISBN 978-3-8376-3426-6

Sandra Meusel

Freiwilliges Engagement

und soziale Benachteiligung

Eine biografieanalytische Studie mit Akteuren in schwierigen Lebenslagen Anerkennung - Macht - Hierarchie

April 20I6, 264 Seiten, kart., 32,99 €,

ISBN 978-3-8376-340I-3

Therese Garstenauer, Thomas Hübel, Klara Löffler (Hg.)

Arbeit im Lebenslauf

Verhandlungen von

(erwerbs-)biographischer Normalität

März 20I6, 212 Seiten, kart., 29,99€,

ISBN 978-3-8376-3373-3

\section{Laura Glauser}

Das Projekt des unternehmerischen Selbst

Eine Feldforschung

in der Coachingzone

März 20I6, 266 Seiten, kart., 34,99€,

ISBN 978-3-8376-342I-I
Alexandra Manske

Kapitalistische Geister

in der Kultur- und

Kreativwirtschaft

Kreative zwischen wirtschaftlichem

Zwang und künstlerischem Drang

2015, 454 Seiten, kart., 39,99€,

ISBN 978-3-8376-2088-7

Carina Großer-Kaya

Biographien der Arbeit -

Arbeit an Biographien

Identitätskonstruktionen

türkeistämmiger Männer

in Deutschland

2015, 330 Seiten, kart., 39,99€,

ISBN 978-3-8376-3239-2

Ilka Sommer

Die Gewalt des kollektiven

Besserwissens

Kämpfe um die Anerkennung

ausländischer Berufsqualifikationen

in Deutschland

20I5, 4I2 Seiten, kart., 39,99€,

ISBN $978-3-8376-3292-7$

Gabriele Fischer

Praktiken der Anerkennung

und Geschlechterdifferenzierung

in der Chirurgie und im Friseurhandwerk

2015, 232 Seiten, kart., 32,99€,

ISBN 978-3-8376-3062-6

Leiv Eirik Voigtländer

Armut und Engagement

Zur zivilgesellschaftlichen

Partizipation von Menschen

in prekären Lebenslagen

2015, 322 Seiten, kart., 34,99€,

ISBN 978-3-8376-3135-7 
